Nevada

Environmental

Restoration

Project

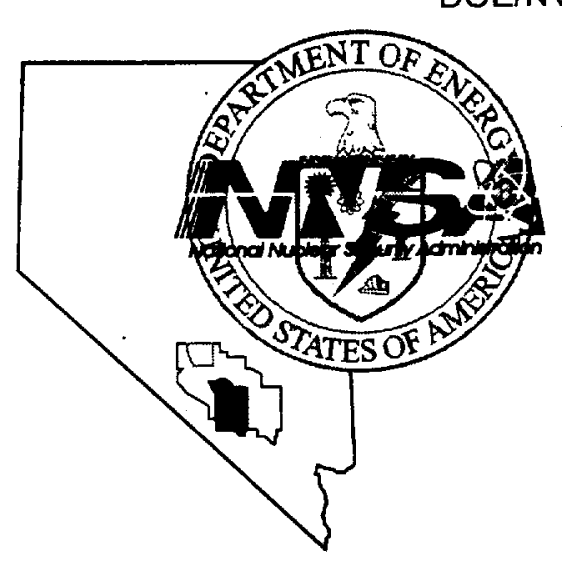

Closure Report for

Corrective Action Unit 230:

Area 22 Sewage Lagoons

And

Corrective Action Unit 320:

Area 22 Desert Rock Airport

Strainer Box

Nevada Test Siter Nevada

Controlled Copy No::

Revision: 0

July 2001

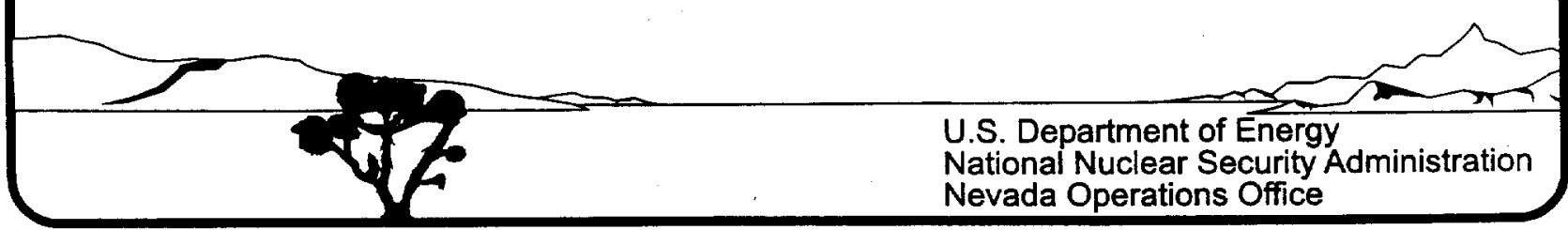


Available for sale to the public, in paper, from:

\author{
U.S. Department of Commerce \\ National Technical Information Service \\ 5285 Port Royal Road \\ Springfield, VA 22161-0002 \\ Telephone: (800) 553-6847 \\ Fax: (703) 605-6900 \\ E-mail: orders@ntis.fedworld.gov \\ Online ordering: http://www.ntis.gov/ordering.htm
}

Available electronically at http://www.doe.gov/bridge

Available for a processing fee to the U.S. Department of Energy and its contractors, in paper, from:

\author{
U.S. Department of Energy \\ Office of Scientific and Technical Information \\ P.O. Box 62 \\ Oak Ridge, TN 37831-0062 \\ Telephone: (865) 576-8401 \\ Fax: (865) 576-5728 \\ E-mail: reports@adonis.osti.gov
}

Reference herein to any specific commercial product, process, or service by trade name, trademark, manufacturer, or otherwise, does not necessarily constitute or imply its endorsement, recommendation, or favoring by the U.S. Government or any agency thereof or its contractors or subcontractors. 


\section{CLOSURE REPORT FOR CORRECTIVE ACTION UNIT 230: AREA 22 SEWAGE LAGOONS \\ AND \\ CORRECTIVE ACTION UNIT 320: \\ AREA 22 DESERT ROCK AIRPORT STRAINER BOX, \\ NEVADA TEST SITE, NEVADA}

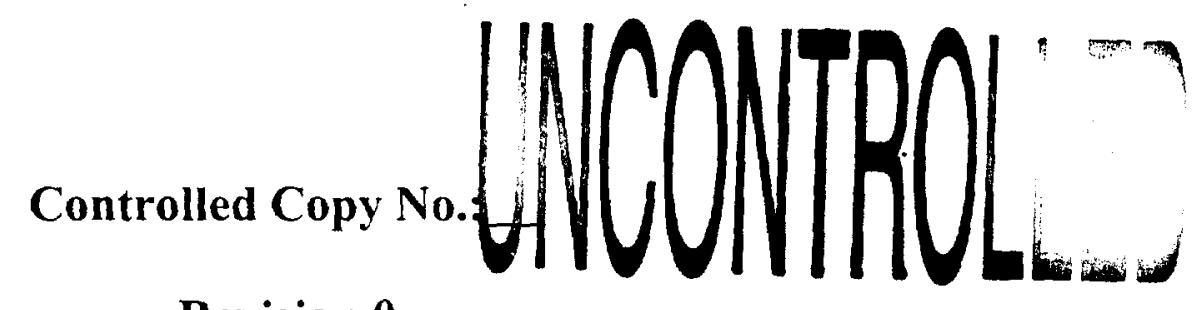
Revision 0

July 2001

Prepared for the U.S. Department of Energy

National Nuclear Security Administration

Nevada Operations Office

under Contract No. DE-AC08-96NV11718 
THIS PAGE INTENTIONALLY LEFT BLANK

4

THIS PAGEINTENTIONALLY LERT BLANK

, 


\section{CLOSURE REPORT FOR CORRECTIVE ACTION UNIT 230: AREA 22 SEWAGE LAGOONS AND CORRECTIVE ACTION UNIT 320: AREA 22 DESERT ROCK AIRPORT STRAINER BOX, NEVADA TEST SITE, NEVADA}

Approved by: $\frac{(\text { Jantet L. Appenzeller-Wing, Project Manager }}{\text { Industrial Sites Project }}$

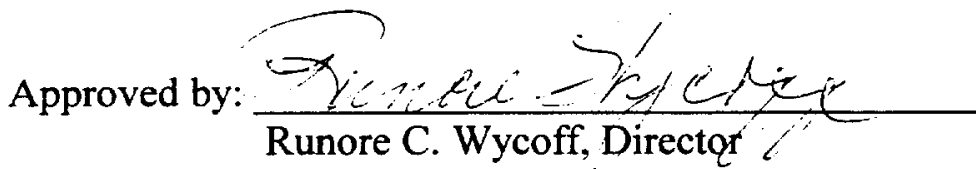

Environmental Restoration Division

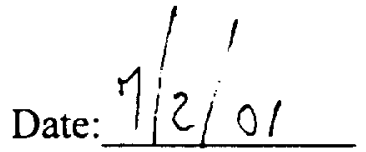


THIS PAGE INTENTIONALLY LEFT BLANK 


\section{TABLE OF CONTENTS}

ACRONYMS AND ABBREVIATIONS $\ldots \ldots \ldots \ldots \ldots \ldots \ldots \ldots \ldots \ldots \ldots \ldots$ vii

EXECUTIVE SUMMARY $\ldots \ldots \ldots \ldots \ldots \ldots \ldots \ldots \ldots \ldots \ldots \ldots \ldots \ldots$

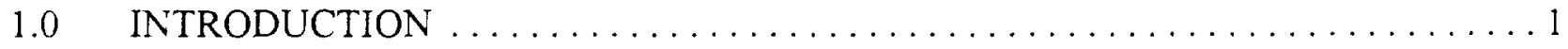

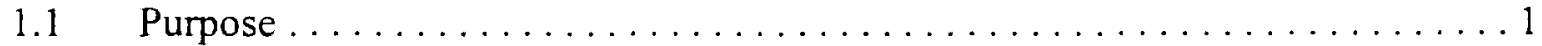

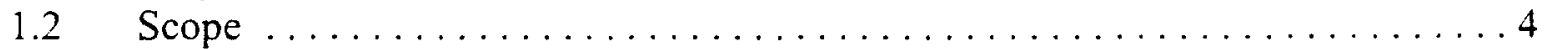

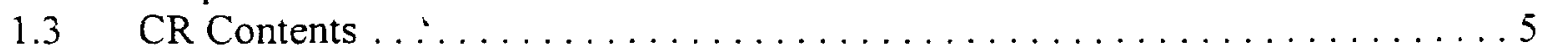

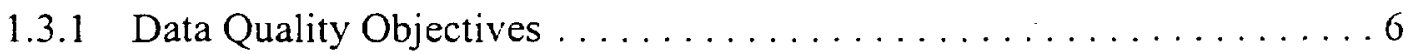

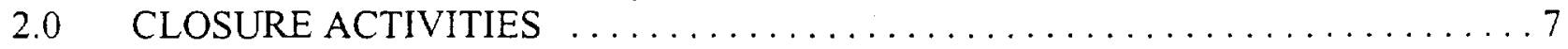

2.1 Description of Corrective Action Activities $\ldots \ldots \ldots \ldots \ldots \ldots \ldots \ldots \ldots$

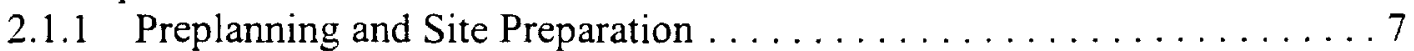

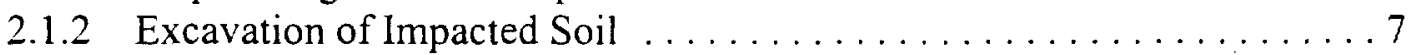

2.1.3 Excavation of Construction Debris $\ldots \ldots \ldots \ldots \ldots \ldots \ldots$

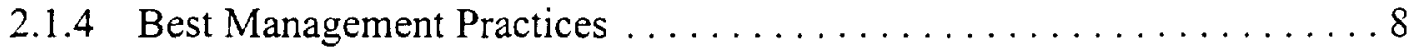

2.1.5 Decontamination of Equipment $\ldots \ldots \ldots \ldots \ldots \ldots \ldots \ldots \ldots$

2.1.6 Backfilling and Regrading of Excavation $\ldots \ldots \ldots \ldots \ldots \ldots$

2.2 Deviations from the CAP as Approved $\ldots \ldots \ldots \ldots \ldots \ldots \ldots \ldots$

2.3 Corrective Action Schedule as Completed $\ldots \ldots \ldots \ldots \ldots \ldots \ldots \ldots \ldots$

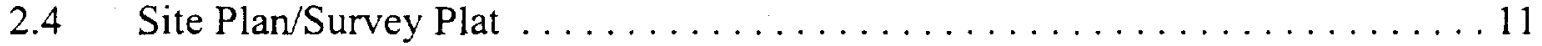

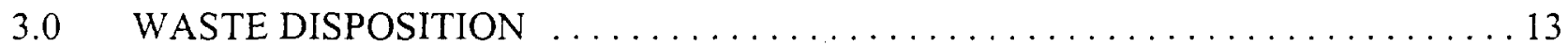

4.0 CLOSURE VERIFICATION RESULTS $\ldots \ldots \ldots \ldots \ldots \ldots \ldots \ldots \ldots \ldots$

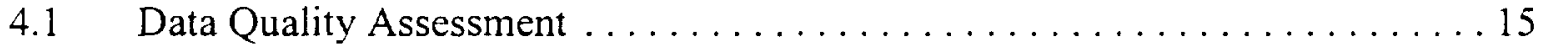

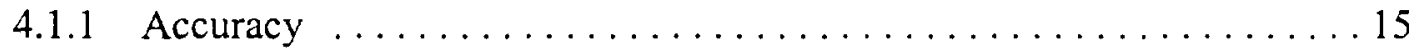

4.1 .2 Precision . . . . . . . . . . . . . . . . . . . . . 16

4.1 .3 Representativeness . . . . . . . . . . . . . . . . . . . . 16

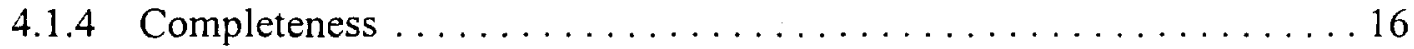

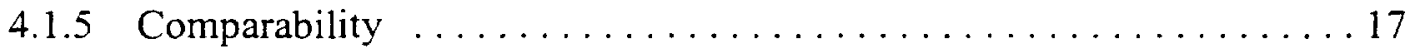

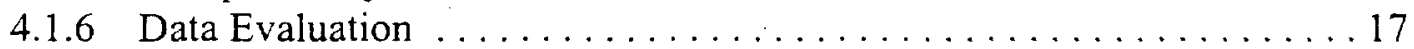

4.1 .7 Quality Control Samples ...................... 17

4.1.8 Laboratory Quality Control Samples . . . . . . . . . . . . . 17

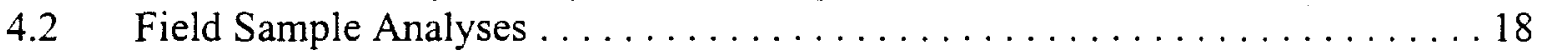

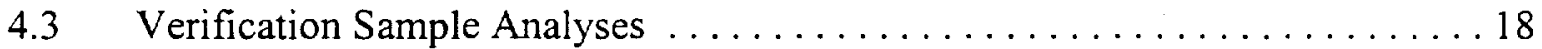

$4.4 \quad$ Use Restriction . . . . . . . . . . . . . . . . . . . . . . 22

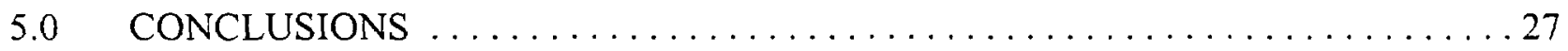

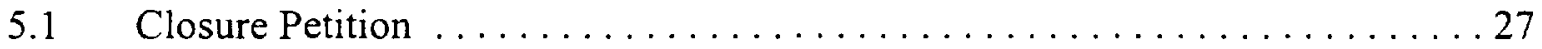

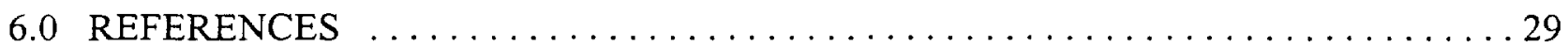




\section{TABLE OF CONTENTS (continued)}

\section{APPENDICES}

Appendix A: Photographs of Closure Activities

Appendix B: Records of Technical Change

Appendix C: Waste Disposition Documentation

Appendix D: Verification Sample Analytical Reports

Appendix E: Comment Review Documentation

DISTRIBUTION LIST

\section{FIGURES}

Figure 1 - CAU $230 / 320$ Location Map ........................ 2

Figure 2 - CAU $230 / 320$ Site Map ........................... 3

Figure 3 - CAU $230 / 320$ Manhole Locations $\ldots \ldots \ldots \ldots \ldots \ldots \ldots \ldots \ldots \ldots \ldots \ldots$

Figure 4 - Closure Schedule as Completed $\ldots \ldots \ldots \ldots \ldots \ldots \ldots \ldots \ldots \ldots \ldots \ldots \ldots \ldots$

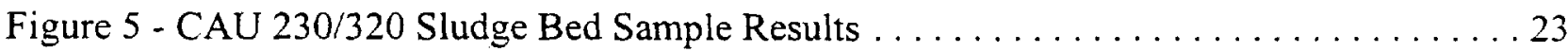

\section{TABLES}

Table 1 - PetroFLAG ${ }^{\mathrm{TM}}$ Sample Results $\ldots \ldots \ldots \ldots \ldots \ldots \ldots \ldots \ldots \ldots \ldots$

Table 2 - Verification Sample Results - March 13, $2001 \ldots \ldots \ldots \ldots \ldots \ldots \ldots$

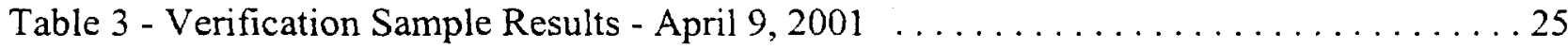




\section{ACRONYMS AND ABBREVIATIONS}

\begin{tabular}{|c|c|}
\hline $\mathrm{BN}$ & Bechtel Nevada \\
\hline $\mathrm{CADD}$ & Corrective Action Decision Document \\
\hline CAIP & Corrective Action Investigation Plan \\
\hline CAP & Corrective Action Plan \\
\hline CAS & Corrective Action Site \\
\hline CAU & Corrective Action Unit \\
\hline $\mathrm{COC}$ & Constituent of Concern \\
\hline $\mathrm{CR}$ & Closure Report \\
\hline $\mathrm{DOE} / \mathrm{NV}$ & U.S. Department of Energy, Nevada Operations Office \\
\hline FFACO & Federal Facility Agreement and Consent Order \\
\hline $\mathrm{ft}$ & feet \\
\hline JHA & Job Hazard Analysis \\
\hline $\mathrm{m}$ & meter \\
\hline $\mathrm{m}^{3}$ & cubic meter \\
\hline $\mathrm{mg} / \mathrm{kg}$ & milligram per kilogram \\
\hline $\mathrm{NAC}$ & Nevada Administrative Code \\
\hline NDEP & Nevada Division of Environmental Protection \\
\hline NNSA/NV & National Nuclear Security Administration Nevada Operations Office \\
\hline NTS & Nevada Test Site \\
\hline ppm & parts per million \\
\hline TPH & Total Petroleum Hydrocarbon \\
\hline$y d^{3}$ & cubic yard \\
\hline
\end{tabular}


THIS PAGE INTENTIONALLY LEFT BLANK 


\section{EXECUTIVE SUMMARY}

The Area 22 Sewage Lagoons, Corrective Action Unit (CAU) 230, and the Area 22 Desert Rock Airport Strainer Box, CAU 320 was clean-closed following the approved Corrective Action Decision Document closure alternative and in accordance with the Federal Facility Agreement and Consent Order (FFACO, 1996). The CAU consists of two Corrective Action Sites (CASs):

- 22-03-01 - Sewage Lagoon (CAU 230)

- 22-99-01 - Strainer Box (CAU 320)

Included with CAS 22-99-01 is a buried Imhoff tank and a sludge bed.

Characterization activities indicated that only the sludge bed (CAS 22-99-01) contained constituents of concern (COCs) above action levels and required remediation. The COC detected was Total Petroleum Hydrocarbons (TPH) as diesel.

The Area 22 Sewage Lagoons site was used for the disposal of sanitary sewage from the Camp Desert Rock Facility and was in operation from 1951 to 1958. It consisted of a buried Imhoff tank (solid/liquid separator), strainer box, sludge bed, sewage lagoons, and associated piping.

The clean-closure corrective action consisted of excavation, disposal, verification sampling, backfilling, and regrading. Best management practices were done at those areas which were found not to have COCs above action levels. This included backfilling and grading the eroded area at the buried Imhoff tank and filling the strainer box and manholes associated with the septic system with a cement/grout slurry.

Closure activities began on January 24, 2001, and ended on April 25, 2001. A total of 869 cubic meters ( 1,136 cubic yards) of impacted soil was excavated and disposed. Construction debris was also removed and disposed. Verification samples were collected from the bottom and sidewalls of the excavation on March 13 and April 9, 2001. Samples were analyzed for TPH full scan and TPH diesel, respectively. The sample results indicated that the impacted soil was related to the asphalt and not a petroleum hydrocarbon spill. Sample results also indicated that all impacted soil above the action level was removed from the bottom of the excavation and the lateral extent of impacted soil was defined. Therefore, with concurrence from the Nevada Division of Environmental Protection (NDEP), not all the asphalt was removed from the sidewalls prior to backfilling. The asphalt does not constitute a hazard to human health or the environment and clean closure with no use restrictions was approved.

The closure was completed following the approved Corrective Action Plan. Waste above the petroleum hydrocarbon action level of $100 \mathrm{mg} / \mathrm{kg}$ was disposed in the Area 6 Hydrocarbon 
Landfill. All construction debris was disposed in the Area 9 Construction Landfill or the Area 23 Sanitary Landfill.

Therefore, the U.S. Department of Energy, National Nuclear Security Administration Nevada Operations Office requests that a Notice of Completion be issued by the NDEP for CAU 230, Area 22 Sewage Lagoons and CAU 320 Area 22 Desert Rock Airport Strainer Box, and that CAU 230/320 be moved from Appendix III to Appendix IV of the FFACO, "Closed Corrective Action Units." Because this is a clean closure, post-closure monitoring is not required at the site. 


\subsection{INTRODUCTION}

This Closure Report (CR) describes the remediation activities performed and the results of verification sampling conducted at Corrective Action Unit (CAU) 230, Area 22 Sewage Lagoons and CAU 320, Area 22 Desert Rock Airport Strainer Box. The CAU is currently listed in Appendix III of the Federal Facility Agreement and Consent Order (FFACO, 1996). The CAU is located in Area 22 of the Nevada Test Site (NTS) (Figure 1) and consists of the following Corrective Action Sites (CASs):

- 22-03-01 - Sewage Lagoon (CAU 230)

22-99-01 - Strainer Box (CAU 320)

Included with CAS 22-99-01 is a buried Imhoff tank and a sludge bed. These CAUs will be collectively referred to in this plan as the Area 22 Sewage Lagoons site.

Site characterization activities were done during September 1999. Characterization of the manholes associated with the septic system leading to the Imhoff tank was done during March 2000. The results of the characterization presented in the Corrective Action Decision Document (CADD) indicated that only the sludge bed (CAS 22-99-01) contained constituents of concern (COC) above action levels and required remediation (U.S. Department of Energy, Nevada Operations Office [DOE/NV], 2000a).

\subsection{PURPOSE}

- The purpose of this CR is to provide documentation of the completed corrective action and to provide data confirming the corrective action. The corrective action was performed in accordance with the approved Corrective Action Plan (CAP) (DOE/NV, 2000b) and consisted of clean closure by excavation and disposal.

The Area 22 Sewage Lagoons site was used for the disposal of sanitary sewage from the Camp Desert Rock Facility and was in operation from 1951 to 1958. It consisted of a buried Imhoff tank (solid/liquid separator), strainer box, sludge bed, sewage lagoons, and associated piping (Figure 2). 


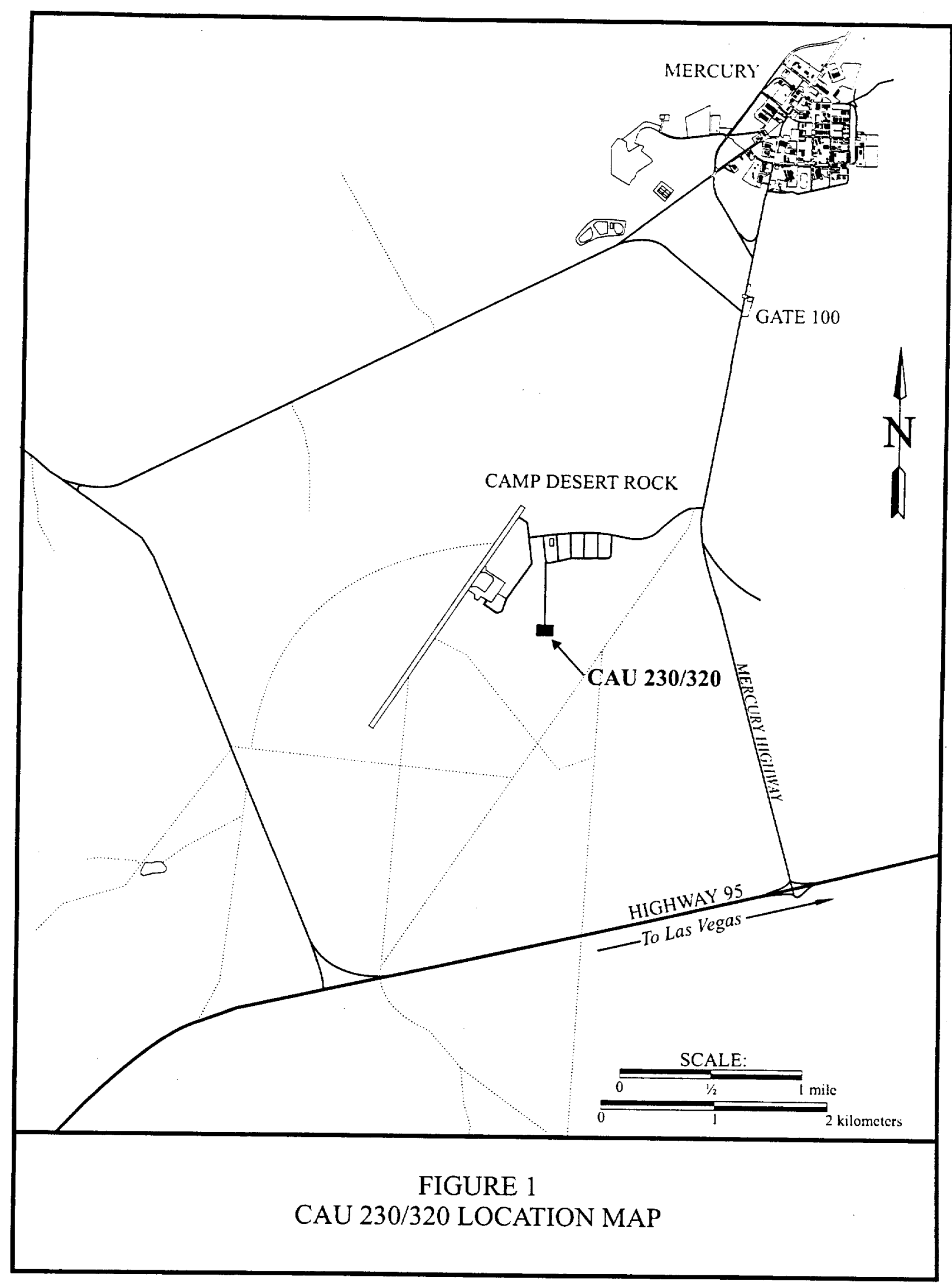




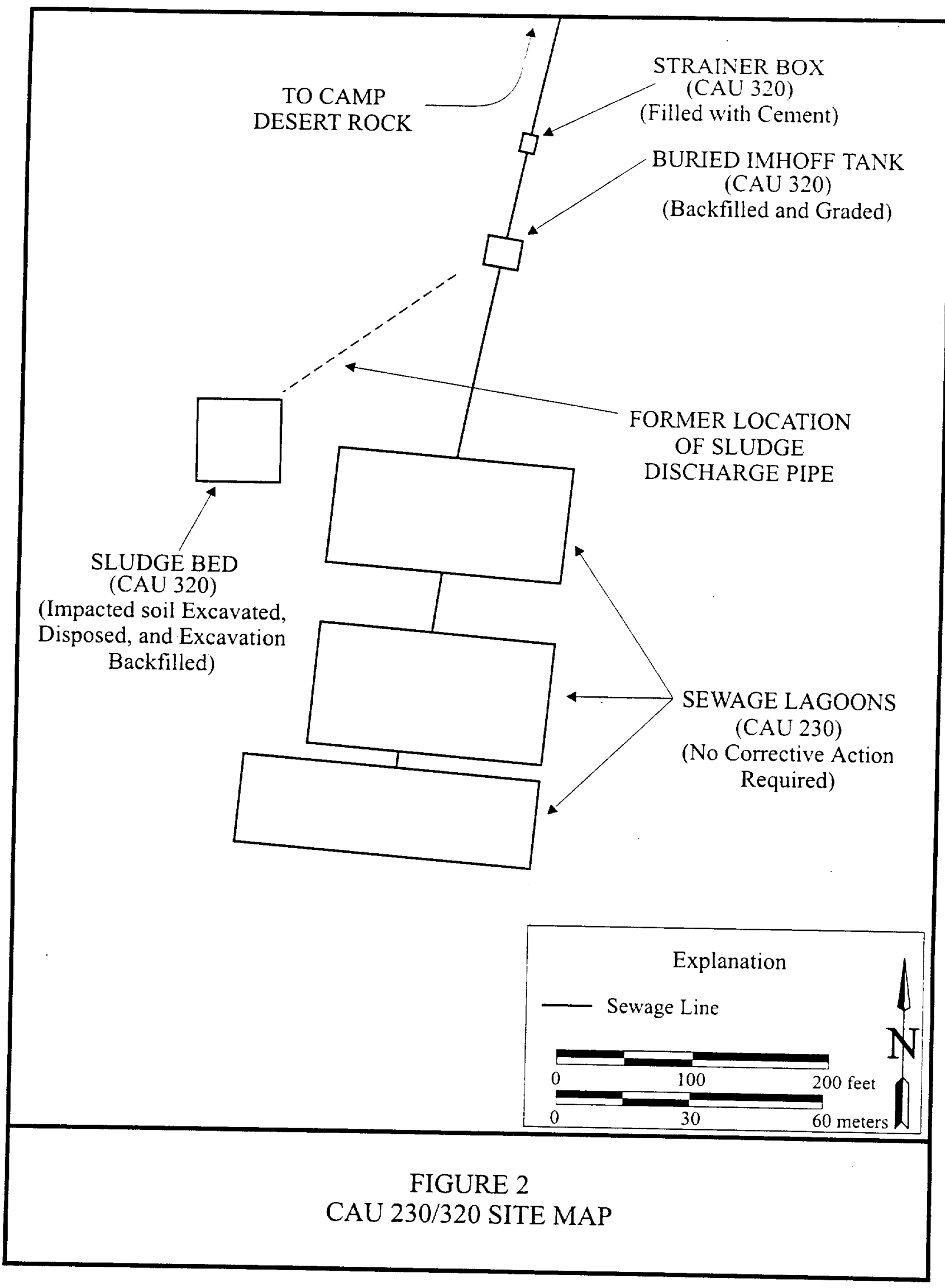


Based on the sample results from the site characterization, the only COC detected was total petroleum hydrocarbons (TPH) as diesel range organics. The sludge bed contained soil with TPH as diesel range organics at levels of up to 580 milligrams per kilogram $(\mathrm{mg} / \mathrm{kg})$, which exceeds the Nevada Division of Environmental Protection (NDEP) regulatory action level of $100 \mathrm{mg} / \mathrm{kg}$ for TPH (Nevada Administrative Code [NAC], 1996).

During closure activities, only asphalt and construction debris was observed and excavated. It was determined through visual inspection, field screening, and verification sampling that the TPH as diesel range organics was associated with the asphalt and not a diesel fuel release. A detailed discussion is provided in Section 4.0.

\subsection{SCOPE}

The scope of work for this project consists of the following activities:

- Preplanning and site preparation, including preparation of plans and permits, delineation of excavation boundaries, and mobilization of equipment and personnel to the site.

- Excavation of soil containing petroleum hydrocarbons above $100 \mathrm{mg} / \mathrm{kg}$ from the sludge bed to a maximum of 15 - by 15 - by 3 - meters $(\mathrm{m})(50$ - by 50 - by 10 - feet [ft]).

- Excavation of construction debris.

- Conduct best management practices at the system components which were found to contain COCs below regulatory levels.

- Collection of soil samples to verify that COCs have been removed to approved levels.

- Disposal of excavated materials following applicable federal, state, and U.S. Department of Energy, National Nuclear Security Administration Nevada Operations Office (NNSA/NV) requirements following Section 2.3 of the CAP (DOENV, 2000b).

- Decontamination of equipment as necessary.

- Backfilling and grading of the site. 


\subsection{CR CONTENTS}

This document is divided into the following sections in accordance with the approved FFACO CR standardized outline:

- Section 1.0 - Introduction

- Section 2.0 - Closure Activities

- Section 3.0 - Waste Disposition

- $\quad$ Section 4.0 - Closure Verification Results

- Section 5.0 - Conclusions

- $\quad$ Section 6.0 - References

Certain sections and appendices of this document have been modified from the approved FFȦCO outline. The following FFACO sections and appendices have not been included or revised as indicated below:

- Use Restriction - Not applicable. The site was clean-closed.

- Closure Certification - Not applicable.

- As-Built Documentation - Not applicable. No engineered structures were constructed.

- Modifications to the Post-Closure Plan - Not applicable. The site was clean-closed.

The appendices included in this document are provided as follows:

- Appendix A: Photographs of Closure Activities

- Appendix B: Records of Technical Change

- Appendix C: Waste Disposition Documentation

- Appendix D: Verification Sample Analytical Reports 


\subsubsection{DATA QUALITY OBJECTIVES}

The data quality objectives (DQOs) used for the clean closure of CAU 230/320 were adapted from the Corrective Action Investigation Plan (CAIP) (DOE/NV, 1999). The characterization activities determined that the only COC was TPH as diesel range organics and was found at the sludge bed (CAS 22-99-01) (DOE/NV, 2000a). A 15- by 15 - by 3-meters (m) (50- by 50 - by 10 - feet [ $\mathrm{ft}]$ ) area was determined to be above the NDEP regulatory action level of $100 \mathrm{mg} / \mathrm{kg}$. Therefore, only the site conceptual model and the TPH relevant sections of the CAIP DQOs (DOE/NV, 1999) are applicable for CAU 230/320 clean closure.

The NDEP approved CAP (DOE/NV, 2000b) documented the location and number of samples required to verify that the all of the TPH impacted soil above the $100 \mathrm{mg} / \mathrm{kg}$ regulatory limit had been removed. A comparison of the verification data to the data quality criteria specified within the CAIP DQOs is provided in Section 4.1. Based on the evaluation provided in Section 4.1, the site conceptual model dimensions had to be modified to the account for the presence of asphalt within the sludge beds. However, all specified verification samples were collected and the analytical data meet the specified DQO quality criteria. 


\subsection{CLOSURE ACTIVITIES}

\subsection{DESCRIPTION OF CORRECTIVE ACTION ACTIVITIES}

\subsubsection{PREPLANNING AND SITE PREPARATION}

Several planning documents were prepared prior to beginning corrective action activities at CAU 230/320. These plans included the CAP (DOE/NV, 2000b), Field Management Plan (Bechtel Nevada [BN], 2000), a Job Hazard Analysis (JHA), and an excavation permit. In addition, a National Environmental Policy Act Checklist was prepared and approved and a site survey was performed by a biologist. Evidence of tortoise habitation or other threatened or endangered species was not found.

Site preparation involved a utility survey, mobilization of equipment to the site, and designation of the remediation zone. There was an active above-ground power line present at the site. However, it did not impact site activities or create a safety hazard. A survey of underground utilities was done so that excavations would not cross any utility lines. No underground utilities were identified.

\subsubsection{EXCAVATION OF IMPACTED SOIL}

Closure activities began on January 24, 2001, with excavation of impacted soil from the sludge bed. Excavation was done with a front-end loader. Excavation proceeded by removing the soil containing visible asphalt as an indicator of impacted soil. Field samples were periodically collected from the bottom and sidewalls of the excavation and screened for hydrocarbon content using a PetroFLAG ${ }^{\mathrm{TM}}$ test kit. Additionally, the field samples with the highest PetroFLAG ${ }^{\mathrm{TM}}$ results were screened for head space analysis. The samples were placed into a sealed plastic bag and placed in the sun for 30 minutes. Head space was then analyzed using a photoionization detector with a lamp energy of 11.4 electron volts. The results of these analyses are provided in Section 4.0 .

Digging stopped when the excavation reached the planned size of 15 - by 15 - by $3-\mathrm{m}$ ( 50 - by 50 by $10-\mathrm{ft})$. A total of approximately 869 cubic meters $\left(\mathrm{m}^{3}\right)\left(1,136\right.$ cubic yards $\left.\left[\mathrm{yd}^{3}\right]\right)$ of hydrocarbons in soil was excavated. The soil was loaded directly into dump trucks and transported to the NTS Area 6 Hydrocarbon Landfill for disposal.

Even though the planned maximum size of the excavation was reached, not all of the asphalt was removed and some of the verification samples results were above the regulatory action level. Discussions were held with the NDEP and NNSANV to determine an appropriate course of action. In order to determine the lateral extent of the waste, additional verification samples were collected higher on the sidewalls than the original samples. Since the sidewalls were sloped, the 
samples were collected further out from the center of the excavation. Additional discussion and verification sample results are provided in Section 4.0.

\subsubsection{EXCAVATION OF CONSTRUCTION DEBRIS}

Construction debris was uncovered during excavation activities. This debris consisted of plastic pipe and conduit, parking ballards, metal plates, barbed wire, wood, concrete, and metal strapping. This debris was loaded into a roll-off container and transported to the NTS Area 9 Construction Landfill for disposal. Approximately $7.6 \mathrm{~m}^{3}\left(10 \mathrm{yd}^{3}\right)$ of construction debris was disposed.

\subsubsection{BEST MANAGEMENT PRACTICES}

Since the remainder of the components associated with the Area 22 Sewage Lagoons Site were found to have COCs below the preliminary action levels, they did not require corrective action. However, best management practices were done at these areas to prevent or mitigate the accumulation of water or debris in the existing piping.

The eroded area at the buried Imhoff tank was backfilled with clean material and graded and the strainer box was filled with a cement/grout slurry (Figure 2). The manholes associated with the septic system were also filled with cement/grout slurry (Figure 3 ).

The sewage lagoons did not require corrective action.

\subsubsection{DECONTAMINATION OF EQUIPMENT}

All hand-held sampling equipment was decontaminated prior to being brought to the field. Soil sampling scoops were decontaminated using a laboratory-grade detergent solution and a deionized water rinse. Hand-held sampling equipment was used only once in the field, so that decontamination in the field was not required.

\subsubsection{BACKFILLING AND REGRADING OF EXCAVATION}

The backfilling operation was completed on April 25, 2001. All clean fill soil was obtained from a source in Area 6 of the NTS. The excavation was backfilled to existing grade, wheelcompacted using a front-end loader and water truck, and graded to minimize ponding of surface water. All remaining fencing and debris were removed and disposed. Photographs of closure activities are provided in Appendix A. 


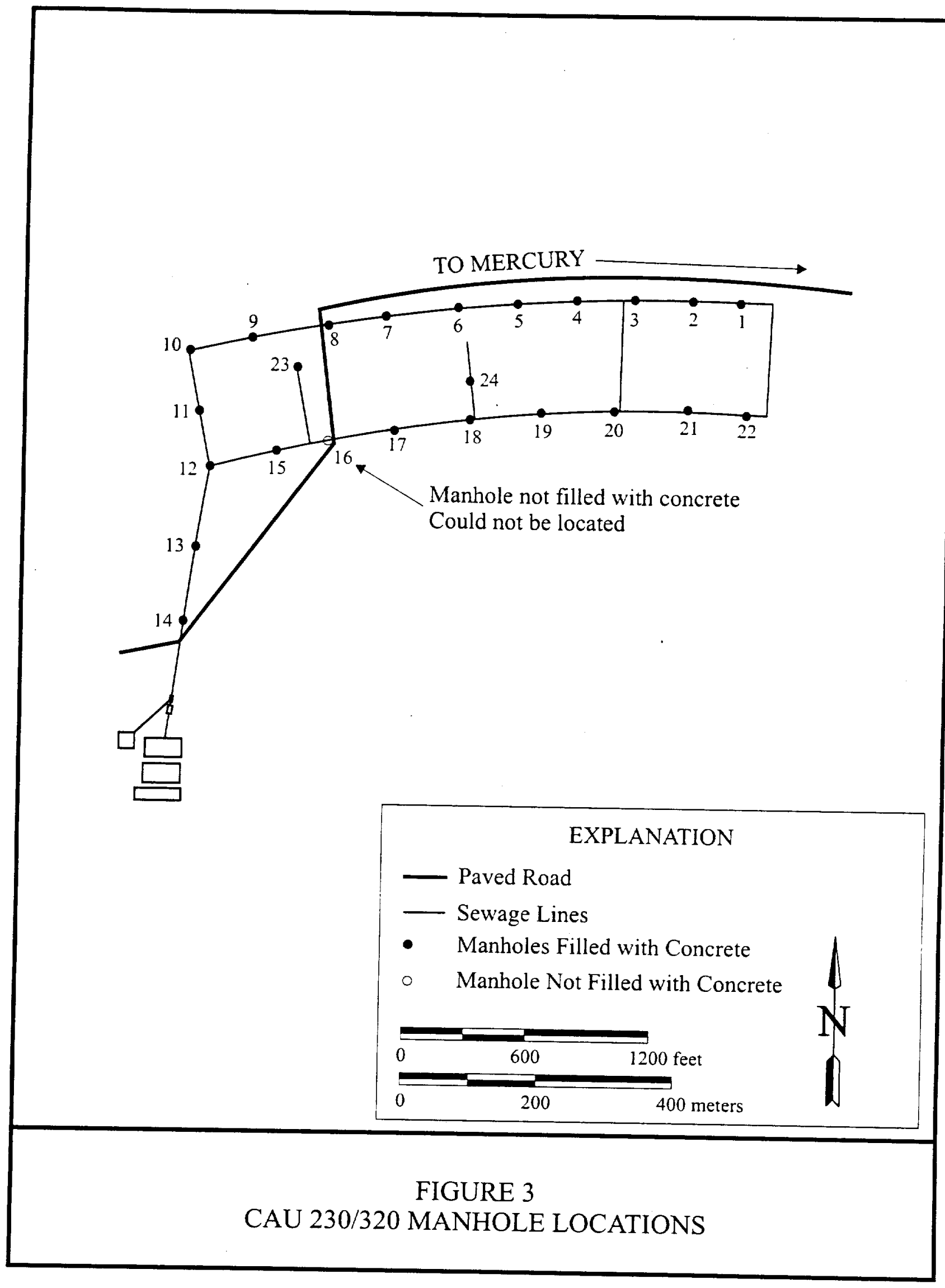




\subsection{DEVIATIONS FROM THE CAP AS APPROVED}

The following deviations occurred from the approved scope of work as outlined in the CAP (DOE/NV, 2000b):

- The site was clean closed by excavation and disposal. However, not all of the asphalt was removed from the sidewalls as proposed in the CAP. Based on sampling and analysis, it was determined that petroleum hydrocarbon waste in the soil was attributed to the asphalt and not a diesel fuel release. The NDEP recognized that the asphalt does not constitute a hazard to human health or the environment and has approved clean closure with no restrictions.

- The actual volume of generated waste varied from the planned volume. A total of $869 \mathrm{~m}^{3}$ $\left(1,136 \mathrm{yd}^{3}\right)$ was disposed rather than the planned $512 \mathrm{~m}^{3}\left(670 \mathrm{yd}^{3}\right)$. The volume increased because more impacted soil than anticipated was found.

- Verification soil samples were collected in less locations than planned. The CAP (DOE/NV, 2000b) proposed collecting 45 verification samples. This was based on the excavation being divided into $3-\mathrm{m}(10-\mathrm{ft})$ grids and collecting a sample from every grid. Instead, a random number generator was used to select five cells from the bottom and two cells from each of the four walls of the excavation. This resulted in the collection of 15 verification samples, including two duplicates. An additional set of 13 verification samples (including one duplicate) was also collected to further define the extent of the impacted soil. Therefore, a total of 28 verification samples was collected. A detailed discussion is presented in Section 4.0. Two technical changes were submitted after verbal approval was granted by the NDEP to conduct the work. The technical changes are provided in Appendix D.

- Twenty-three of the twenty-four manholes associated with the septic system were filled with cement/grout slurry. Manhole Number 16 was not filled because it could not be located. The apparent location of the manhole is near the intersection of the present day Desert Rock Airport Access Road and the historic Camp Desert Rock Road (Figure 3). Therefore, it is likely that the manhole was graded over or removed entirely during road construction.

- A JHA was used for the safety documentation rather than a Site-Specific Health and Safety Plan. After the CAP was approved, it was determined that based on the low levels of TPH as diesel present at the site, there was minimal risk to workers from exposure to diesel. Therefore, only a JHA was required.

- Excavated construction debris was disposed in the NTS Area 9 Construction landfill rather than the NTS Area 6 Hydrocarbon landfill as stated in the CAP. Since the petroleum hydrocarbons are asphalt-related, disposal of this debris in the Area 9 Construction landfill was appropriate. 


\subsection{CORRECTIVE ACTION SCHEDULE AS COMPLETED}

The corrective action field activities began on January 24, 2001, and were completed on April 25, 2001. A corrective action schedule as completed is provided in Figure 4.

\subsection{SITE PLAN/SURVEY PLAT}

Because this is a clean closure, survey data are not required for this closure. Because engineered construction was not required as part of this closure, as-built drawings are not included in this CR. 
FIGURE 4 - CLOSURE SCHEDULE AS COMPLETED

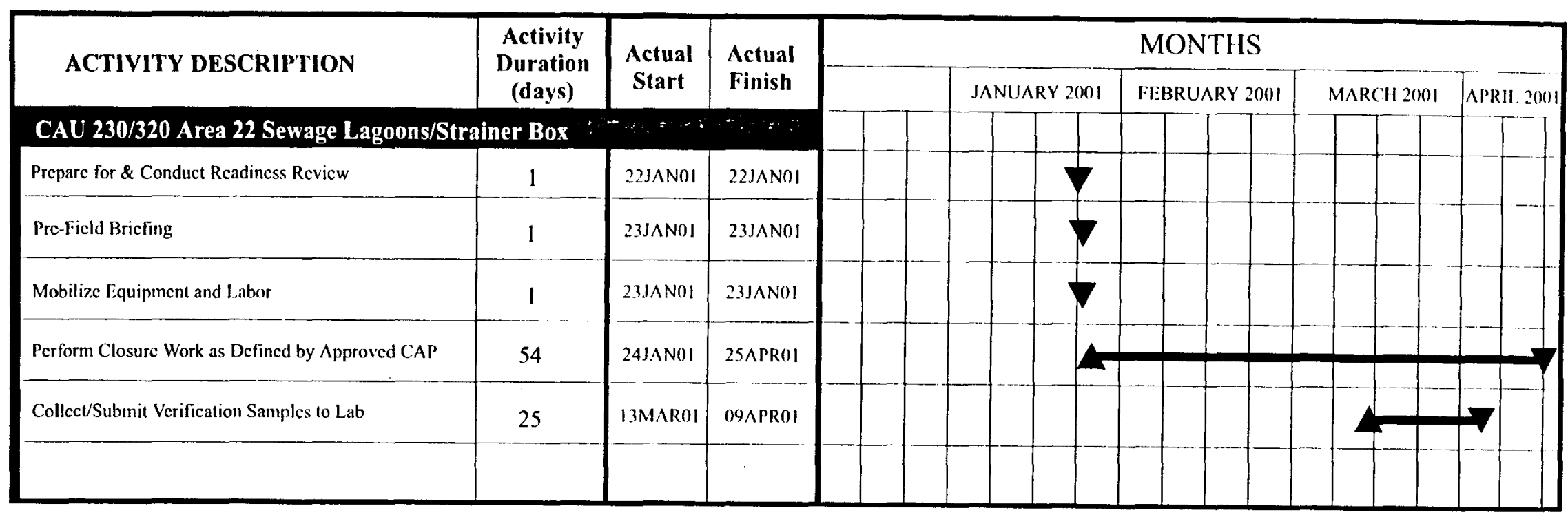




\subsection{WASTE DISPOSITION}

Wastes generated during the closure of CAU 230/320 were disposed as follows:

Approximately $869 \mathrm{~m}^{3}\left(1,136 \mathrm{yd}^{3}\right)$ of asphalt-impacted soil was disposed in the NTS Area 6 Hydrocarbon Landfill.

Construction debris, including plastic pipe and conduit, parking ballards, metal plates, barbed wire, wood, concrete, and metal strapping, was disposed in the Area 9 Construction Landfill. Sanitary trash was disposed in the Area 23 Landfill.

Waste disposal activities were completed on March 21, 2001.

Waste disposition records are provided in Appendix C. 



\subsection{CLOSURE VERIFICATION RESULTS}

Verification sampling was done on March 13 and April 9, 2001 following Section 2.4 of the CAP (DOE/NV, 2000b). The laboratory data packages are provided in Appendix D.

\subsection{DATA QUALITY ASSESSMENT}

A verification program needs to contain the following three elements:

- The verification program must support the field decision that any remaining COCs are less than remediation standards (i.e., when has all of the contamination been remediated).

- The verification program must include a sufficient number of samples to adequately assess the completeness of the remedial action (i.e., is the site really clean).

- Does the verification analytical data collected, meet the quality requirements specified in the DQO.

The following sections discuss the results of the QA/QC data for CAU 230/320 corrective action sampling. Accuracy, precision, representativeness, completeness, and comparability are used in the DQO process to measure quality control and are presented below.

\subsubsection{ACCURACY}

Accuracy is the closeness of a measurement or the mean of a set of results to the true value. Accuracy is a measure of the bias of the measurement system. Indicators for measurement are based on the percent recoveries associated the laboratory analytical control spikes, surrogate spikes, or matrix spikes. Accuracy is then determined by calculation of the percent recovery $(\% \mathrm{R})$ which is the amount recovered divided by the actual amount added times 100 percent.

In addition there are legal requirements involved in accuracy. Samples must be collected at the correct locations with carefully recorded dates and times. This is done through the use of a chain of custody form with custody tape attached to prevent tampering. The chain of custody form follows the samples to the contract laboratory to prove samples were not tampered with and to show that the required hold times were not exceeded. The temperature inside the ice chest used to ship samples must be maintained at $4^{\circ} \mathrm{C} \pm 2^{\circ} \mathrm{C}$. This is checked before shipment and upon receipt by the contract laboratory. 
All samples collected for this project were properly collected and sent to the contract laboratory as described in the Corrective Action Plan.

\subsubsection{PRECISION}

Precision is a measurement which represents the repeatability of the analytical system. Indicators for measurement are based on field duplicates, laboratory splits, or laboratory replicate analysis. It is usually expressed as the relative percent difference (RPD) or standard deviation. Three blind replicates were collected as described in the Corrective Action Plan.

\subsubsection{REPRESENTATIVENESS}

Representativeness is a qualitative judgement which refers to a sample or group of samples that reflect the characteristics of the media at the sampling point. It also includes how well the sampling point represents the actual parameter variations which are under study.

For CAU 230/320 professional judgement, process knowledge, and historical knowledge were used to set the number of samples needed to sufficiently determine that the site was cleaned of hydrocarbons. The closure of CAU 230/320 was complicated by the presences of large quantities of asphalt within the debris that had been backfilled in the sludge bed. The presence of asphalt within the verification samples required an expansion of the planned excavation to get outside of the asphalt impacted soil. Asphalt contains TPH constituents and when analyzed following EPA method 8015 modified, elevated TPH results were obtained. The presence of asphalt within the March 13, 2001 verification samples resulted in extending the excavation and collection of additional samples on April 9, 2001.

\subsubsection{COMPLETENESS}

Indicators for this measurement is the amount of valid data obtained from a measurement system compared to the amount that was expected and needed to be obtained to meet the project data goals.

All samples collected for this project had acceptable QA/QC. In addition, all hold times were met, giving this project a 100 percent completeness. 


\subsubsection{COMPARABILITY}

Comparability is a qualitative judgement which expresses the confidence with which one data set can be compared to another. Items used to determine comparability include the analytical method and reporting units. Standardized methods for collection of environmental samples were used to perform sampling. Samples were analyzed for TPH using the standard method (8015 modified). This allows the data to be compared to other results obtained for other projects or over time.

\subsubsection{DATA EVALUATION}

Laboratory data obtained for CAU 230/320 have been evaluated for data quality. Data was evaluated using the following criteria:

- Chain of custody is complete and legible.

- Cover letter (case narrative) reviewed for significant problems.

- All requested analyses were performed on all samples.

- Holding times are not beyond specified standards.

- Note cooler temperature on cooler is not above 6 degrees Celsius.

- Was the proper preservation and $\mathrm{pH}$ for each Matrix used?

- Check the laboratory log-in report for completeness and errors.

- All field forms are present and complete.

- Report forms inventory includes all CLP or CLP-like forms.

- Reporting values are at the appropriate level.

\subsubsection{QUALITY CONTROL SAMPLES}

Three duplicates and one equipment blank were collected and sent "blind" to the contract laboratory.

\subsubsection{LABORATORY QUALITY CONTROL SAMPLES}

Each sample delivery group (SDG) has a matrix spike/matrix spike duplicate (MS/MSD), laboratory control spike/laboratory control spike duplicate (LCS/LCSD), and method blank. A surrogate spike was also added to each sample. This data was used to qualify the associated sample results.

Surrogates check each environmental sample to determine if the extraction and analysis procedure was performed correctly and if the instrumentation is operating as expected. 
Surrogates are compounds which are highly unlikely to be present in the environment, but have been determined to be similar in behavior to the COCs. The surrogate is added before extraction is started, and the amount recovered is used to calculate percent recovery.

The acceptable range for surrogate recovery is determined by each laboratory. For TPH analysis the laboratory is not required to reanalyze the sample. The laboratory is only required to report the acceptable range and percent recovery.

\subsection{FIELD SAMPLE ANALYSES}

All samples were collected with decontaminated stainless steel sampling scoops and placed in appropriate sample containers. All samples were labeled with a unique sample number, placed on ice in coolers, and transported under chain-of-custody to Environmental Technical Services in Mercury, Nevada for shipment to an off-site laboratory.

Samples were numbered using the following nomenclature:

\section{SB-1}

Where:

- 320 is the CAU number.

- $\quad \mathrm{SB}$ is the site location (Sludge Bed).

- 1 is the sample number.

During excavation, field samples of the soil were collected and screened for hydrocarbon content using a PetroFLAG ${ }^{\mathrm{TM}}$ test kit. The results are provided in Table 1. In addition, the field samples with the highest PetroFLAG ${ }^{\mathrm{TM}}$ results were screened for head space analysis. The samples were placed into a sealed plastic bag and placed in the sun for 30 minutes. Head space was then analyzed using a photoionization detector with a lamp energy of 11.4 electron volts. The result of these analyses was 0.0 parts per million (ppm).

\subsection{VERIFICATION SAMPLE ANALYSES}

A total of 15 verification soil samples were collected on March 13, 2001. These samples were analyzed for full scan TPH because of the concern that the elevated PetroFLAG ${ }^{\mathrm{TM}}$ results may have been due to a diesel fuel spill rather than from the asphalt. Verification soil samples were collected from the sidewalls and floor of the excavation approximately every $1.5 \mathrm{~m}(5 \mathrm{ft})$. 
TABLE 1 - PETROFLAG'M SAMPLE RESULTS

\begin{tabular}{|c|c|c|c|}
\hline SAMPLE ID & SAMPLE LOCATION & SAMPLE DEPTH $(\mathrm{ft})^{A}$ & RESULT $(\mathrm{ppm})^{\mathrm{R}}$ \\
\hline PF1-1 & Southeast Corner Bottom & 7 & 59 \\
\hline PF $1-2$ & Southwest Corner Bottom & 7 & 192 \\
\hline PF 1-3 & Bottom Center & 7 & 148 \\
\hline PF 1-4* & Northeast Corner Bottom & 7 & 1596 \\
\hline PF $1-5^{*}$ & Northwest Corner Bottom & 7 & 1058 \\
\hline PF1-6 & South Sidewall (East) & 5 & 454 \\
\hline PF1-7 & South Sidewall (West) & 5 & 54 \\
\hline PF1-8 & West Sidewall (South) & 5 & 384 \\
\hline PF1-9 & West Sidewall (North) & 5 & 131 \\
\hline PF1-10* & North Sidewall (West) & 5 & 1022 \\
\hline PF1-11 & North Sidewall (East) & 5 & 218 \\
\hline PF 1-12* & East Sidewall (North) & 5 & 2073 \\
\hline PF1-13 & East Sidewall (South) & 5 & 132 \\
\hline
\end{tabular}


TABLE 1 (Continued) - PETROFLAG ${ }^{\text {M }}$ SAMPLE RESULTS

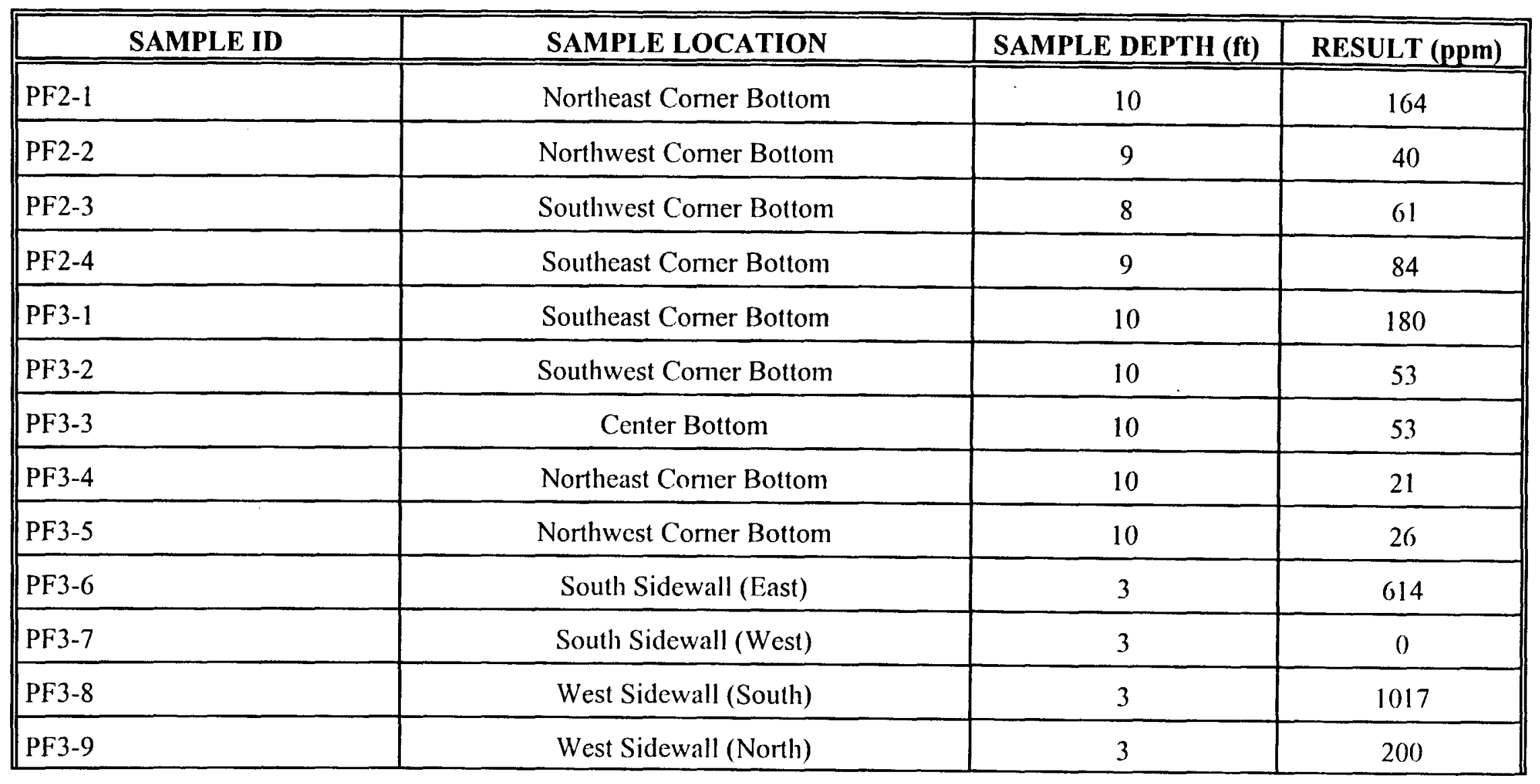


TABLE 1 (Continued) - PETROFLAGTM SAMPLE RESULTS

\begin{tabular}{|l|c|c|c||}
\hline \multicolumn{1}{|c|}{ SAMPLE ID } & SAMPLE LOCATION & SAMPLE DEPTH (ft) & RESULT (pPm) \\
\hline \hline PF3-10 & North Sidewall (West) & 3 & 426 \\
\hline PF3-11 & North Sidewall (East) & 3 & 168 \\
\hline PF3-12 & East Sidewall (North) & 3 & 720 \\
\hline PF3-13 & East Sidewall (South) & 3 & 1293 \\
\hline
\end{tabular}

A - feet

$\mathrm{B}$ - parts per million

* - samples were also analyzed for head space using a PID 
An additional 13 verification soil samples were collected on April 9, 2001. These samples were collected to comply with a request form the NDEP to determine the lateral extent of the hydrocarbon-impacted soil (Gustafson, 2001). These samples were analyzed for TPH as diesel. In addition, two samples of asphalt were collected and analyzed for TPH as diesel. The chromatograms from the asphalt samples were compared to the chromatograms of the soil samples to determine if the waste in the soil was related to asphalt or a diesel fuel spill. The comparison of the chromatograms indicated that the soil waste was asphalt-related. The laboratory data packages for all samples, including the chromatograms, are provided in Appendix D. Sample locations are provided in Figure 5. Results are shown in Tables 2 and 3.

\subsection{USE RESTRICTION}

A clean closure was performed at this CAS. Land use is unrestricted. Because the CAU has been clean closed, a Post-Closure Plan is not necessary. 

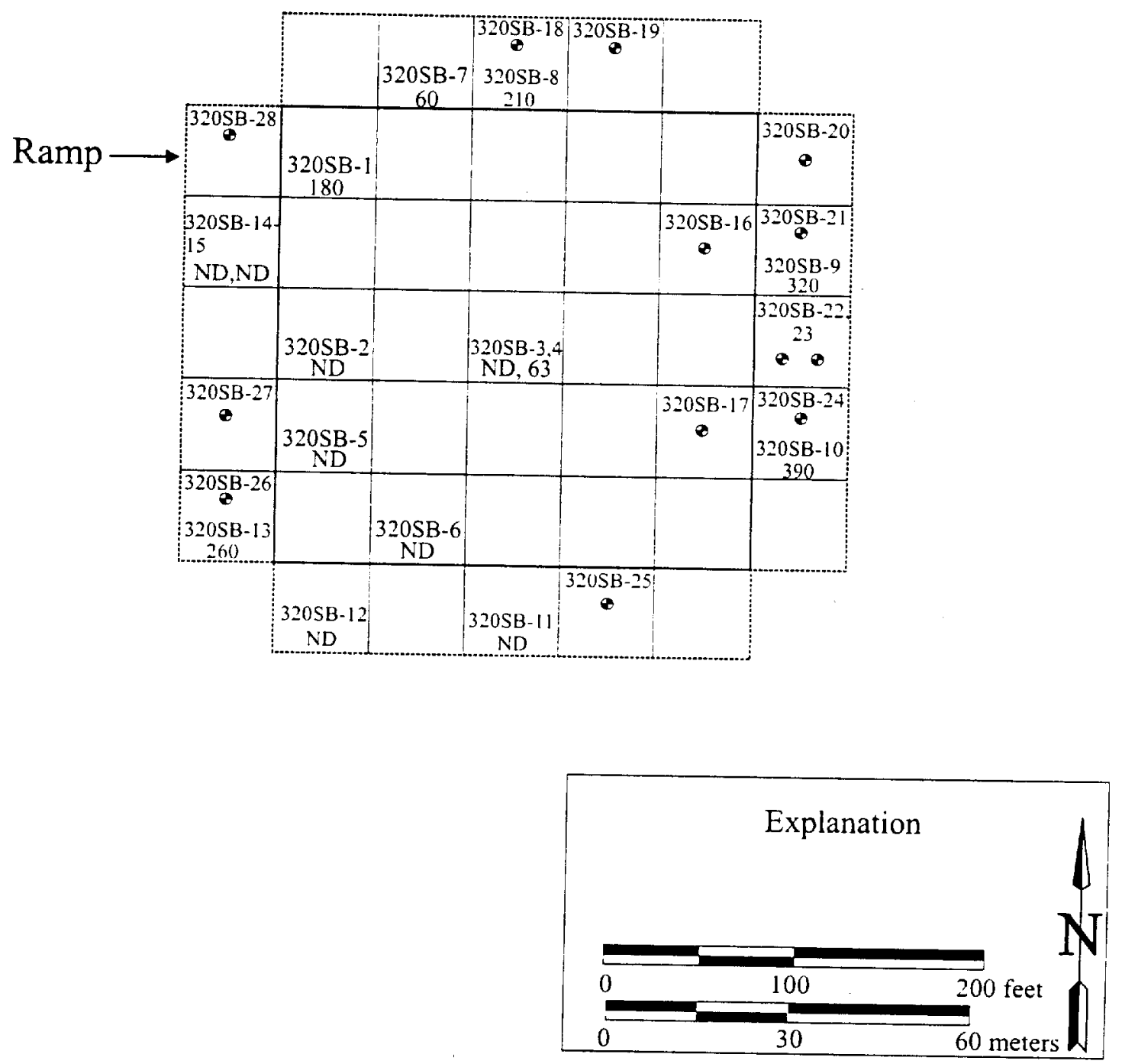

Samples collected 3/13/2001. Results are in milligrams per kilogram $(\mathrm{mg} / \mathrm{kg})$. $\mathrm{ND}=$ Non-detect. Squares with two results indicate duplicate sample.

- Locations of samples collected 4/9/2001. Results were all non-detect for TPH diesel. Squares with two indicates duplicate sample.

FIGURE 5

CAU 230/320 SLUDGE BED SAMPLE RESULTS 
TABLE 2 - VERIFICATION SAMPLE RESULTS - MARCH 13, 2001

\begin{tabular}{|l|c|c|c|}
\hline \multicolumn{1}{|c|}{ SAMPLE ID } & SAMPLE LOCATION & TPII GASOLINE (mg/kg) & TPH DIESEL/OIL(mg/kg) \\
\hline \hline 320 SB-1 & Bottom Grid 1 & $<0.25$ & 180 \\
\hline 320 SB-2 & Bottom Grid 11 & $<0.25$ & $<20$ \\
\hline 320 SB-3 & Bottom Grid 13 & $<0.25$ & 63 \\
\hline 320 SB-4 & Bottom Grid 13 & $<0.25$ & $<20$ \\
\hline 320 SB-5 & Bottom Grid 20 & $<0.25$ & $<20$ \\
\hline 320 SB-6 & Bottom Grid 22 & $<0.25$ & $<20$ \\
\hline 320 SB-7 & North Sidewall Grid 2 & $<0.25$ & 60 \\
\hline 320 SB-8 & North Sidewall Grid 3 & $<0.25$ & 210 \\
\hline 320 SB-9 & East Sidewall Grid 2 & $<0.25$ & 320 \\
\hline 320 SB-10 & East Sidewall Grid 4 & $<0.25$ & 390 \\
\hline 320 SB-11 & South Sidewall Grid 3 & $<0.25$ & $<20$ \\
\hline 320 SB-12 & South Sidewall Grid 5 & $<0.25$ & $<20$ \\
\hline 320 SB-13 & West Sidewall Grid 1 & $<0.25$ & 260 \\
\hline 320 SB-14 & West Sidewall Grid 4 & $<0.25$ & $<20$ \\
\hline 320 SB-15 & West Sidewall Grid 4 & $<0.25$ & $<20$ \\
\hline
\end{tabular}

A - milligrams per kilogram

B - The NDEP action level for petroleum hydrocarbons is $100 \mathrm{mg} / \mathrm{kg}$ (NAC, 1996)

Refer to Figure 5 for sample locations and Appendix D for Laboratory Data Reports 
TABLE 3 - VERIFICATION SAMPLE RESULTS - APRIL 9, 2001

\begin{tabular}{||l|c|c|}
\hline \multicolumn{1}{|c|}{ SAMPLE ID } & SAMPLE LOCATION & TPH DIESEL (mg/kg) \\
\hline \hline 320 SB B 16 & Bottom Grid 6 & $<10$ \\
\hline 320 SB-17 & Bottom Grid 16 & $<10$ \\
\hline 320 SB-18 & North Sidewall Grid 3 & $<10$ \\
\hline 320 SB-19 & North Sidewall Grid 4 & $<10$ \\
\hline 320 SB-20 & East Sidewall Grid 1 & $<10$ \\
\hline 320 SB-21 & East Sidewall Grid 2 & $<10$ \\
\hline 320 SB-23 & East Sidewall Grid 3 & $<10$ \\
\hline 320 SB-24 & East Sidewall Grid 3 & $<10$ \\
\hline 320 SB-25 & East Sidewall Grid 4 & $<10$ \\
\hline 320 SB-26 & South Sidewall Grid 2 & $<10$ \\
\hline 320 SB-27 & West Sidewall Grid 1 & $<10$ \\
\hline 320 SB-28 & West Sidewall Grid 2 & $<10$ \\
\hline \hline
\end{tabular}

A - milligrams per kilogram

B - The NDEP action level for petroleum hydrocarbons is $100 \mathrm{mg} / \mathrm{kg}$ (NAC, 1996)

Refer to Figure 5 for sample locations and Appendix D for Laboratory Data Reports 
THIS PAGE INTENTIONALLY LEFT BLANK 


\subsection{CONCLUSIONS}

CAU 230, Area 22 Sewage Lagoons and CAU 320 Area 22 Desert Rock Airport Strainer Box, include CAS 22-99-01 (Sludge Bed and buried Imhoff tank). The Sludge Bed area was excavated and found to contain asphalt-impacted soil and construction debris. Most of the asphalt-impacted soil was excavated and disposed in the NTS Area 6 Hydrocarbon Landfill. Approval was granted by the NDEP to leave some asphalt in the Sludge Bed because it was determined that impacted soil was related to the asphalt and not a diesel fuel spill. The asphalt and asphalt-impacted soil was disposed in the NTS Area 6 Hydrocarbon Landfill and the construction debris was disposed in the NTS Area 9 Construction Landfill. The excavation was backfilled with clean soil, wheel-compacted, and graded. The eroded area around the buried Imhoff tank was backfilled and graded. The Strainer box and manholes were filled with cement/grout slurry. Verification samples were collected and the analytical results indicated that the extent of the impacted soil was defined and was related to the asphalt. All fencing around the Sludge Bed and Imhoff tank area was removed.

Closure was accomplished in accordance with the approved CAP (DOE/NV, 2000b).

Verification sample data demonstrate that all COCs were removed to the remediation standards. Therefore, the site is clean-closed.

\subsection{CLOSURE PETITION}

This closure was completed following the approved CAP (DOE/NV, 2000b). Based on the completion of site closure activities, the NNSANV requests that a Notice of Completion be issued by the NDEP for CAU 230, Area 22 Sewage Lagoons and CAU 320 Area 22 Desert Rock Airport Strainer Box, and that CAU 230/320 be moved from Appendix III to Appendix IV of the FFACO, "Closed Corrective Action Units." 
THIS PAGE INTENTIONALLY LEFT BLANK 


\subsection{REFERENCES}

BN, see Bechtel Nevada.

Bechtel Nevada, 2000, Field Management Plan for Corrective Action Unit 230: Area 22 Sewage Lagoons and Corrective Action Unit 320: Area 22 Desert Rock Airport Strainer Box, Nevada Test Site, Nevada, Las Vegas, NV.

DOE/NV, see U.S. Department of Energy, Nevada Operations Office.

FFACO, see Federal Facility Agreement and Consent Order.

Federal Facility Agreement and Consent Order. 1996 (as amended). Agreed to by the State of Nevada, the U.S. Department of Energy, and the U.S. Department of Defense.

Gustafson, Dennis. 2001. E-mail to Sabine Curtis, April 4.

NAC, see Nevada Administrative Code.

Nevada Administrative Code, 1996, NAC 445A.2272, "Contamination of soil: Establishment of action levels." As adopted by the Nevada Environmental Commission, September, Carson City, NV.

U.S. Department of Energy, Nevada Operations Office, 1999, Corrective Action Investigation Plan for Corrective Action Unit 230: Area 22 Sewage Lagoons and Corrective Action 320: Area 22 Desert Rock Airport Strainer Box, Nevada Test Site, Nevada, DOE/NV-552, Las Vegas, NV.

U.S. Department of Energy, Nevada Operations Office, 2000a, Corrective Action Decision Document for Corrective Action Unit 230: Area 22 Sewage Lagoons and Corrective Action 320: Area 22 Desert Rock Airport Strainer Box. Nevada Test Site, Nevada, DOE/NV-601, Las Vegas, NV.

U.S. Department of Energy, Nevada Operations Office, 2000b, Corrective Action Plan for Corrective Action Unit 230: Area 22 Sewage Lagoons and Corrective Action Unit 320: Area 22 Desert Rock Airport Strainer Box, Nevada Test Site. Nevada, DOE/NV-655, Las Vegas, NV. 


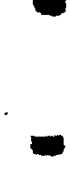

THIS PAGE INTENTIONALLY LEFT BLANK 
APPENDIX A

\section{PHOTOGRAPHS OF}

CLOSURE ACTIVITIES 
THIS PAGE INTENTIONALLY LEFT BLANK 


\subsection{REFERENCES}

BN, see Bechtel Nevada.

Bechtel Nevada, 2000, Field Management Plan for Corrective Action Unit 230: Area 22 Sewage Lagoons and Corrective Action Unit 320: Area 22 Desert Rock Airport Strainer Box, Nevada Test Site, Nevada, Las Vegas, NV.

DOE/NV, see U.S. Department of Energy, Nevada Operations Office.

FFACO, see Federal Facility Agreement and Consent Order.

Federal Facility Agreement and Consent Order. 1996 (as amended). Agreed to by the State of Nevada, the U.S. Department of Energy, and the U.S. Department of Defense.

Gustafson, Dennis. 2001. E-mail to Sabine Curtis, April 4.

NAC, see Nevada Administrative Code.

Nevada Administrative Code, 1996, NAC 445A.2272, "Contamination of soil: Establishment of action levels." As adopted by the Nevada Environmental Commission, September, Carson City, NV.

U.S. Department of Energy, Nevada Operations Office, 1999, Corrective Action Investigation Plan for Corrective Action Unit 230: Area 22 Sewage Lagoons and Corrective Action 320: Area 22 Desert Rock Airport Strainer Box. Nevada Test Site, Nevada, DOE/NV-552, Las Vegas, NV.

U.S. Department of Energy, Nevada Operations Office, 2000a, Corrective Action Decision Document for Corrective Action Unit 230: Area 22 Sewage Lagoons and Corrective Action 320: Area 22 Desert Rock Airport Strainer Box, Nevada Test Site, Nevada, DOE/NV-601, Las Vegas, NV.

U.S. Department of Energy, Nevada Operations Office, 2000b, Corrective Action Plan for Corrective Action Unit 230: Area 22 Sewage Lagoons and Corrective Action Unit 320: Area

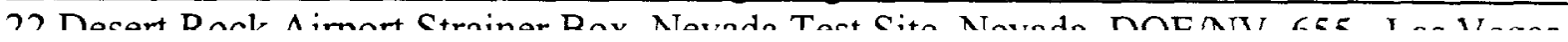


THIS PAGE INTENTIONALLY LEFT BLANK 


\section{APPENDIX A}

\section{PHOTOGRAPHS OF CLOSURE ACTIVITIES}


THIS PAGE INTENTIONALLY LEFT BLANK 


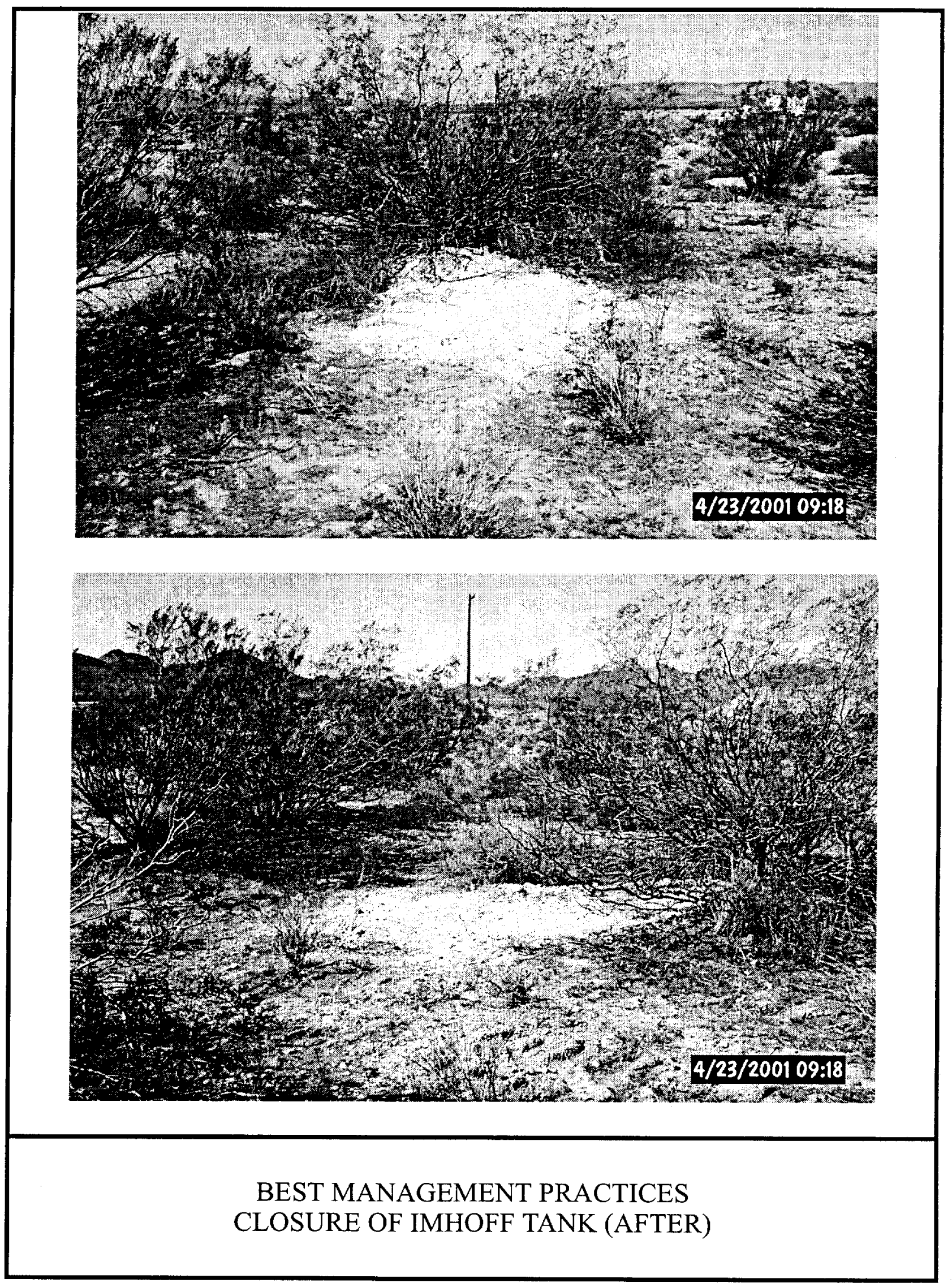




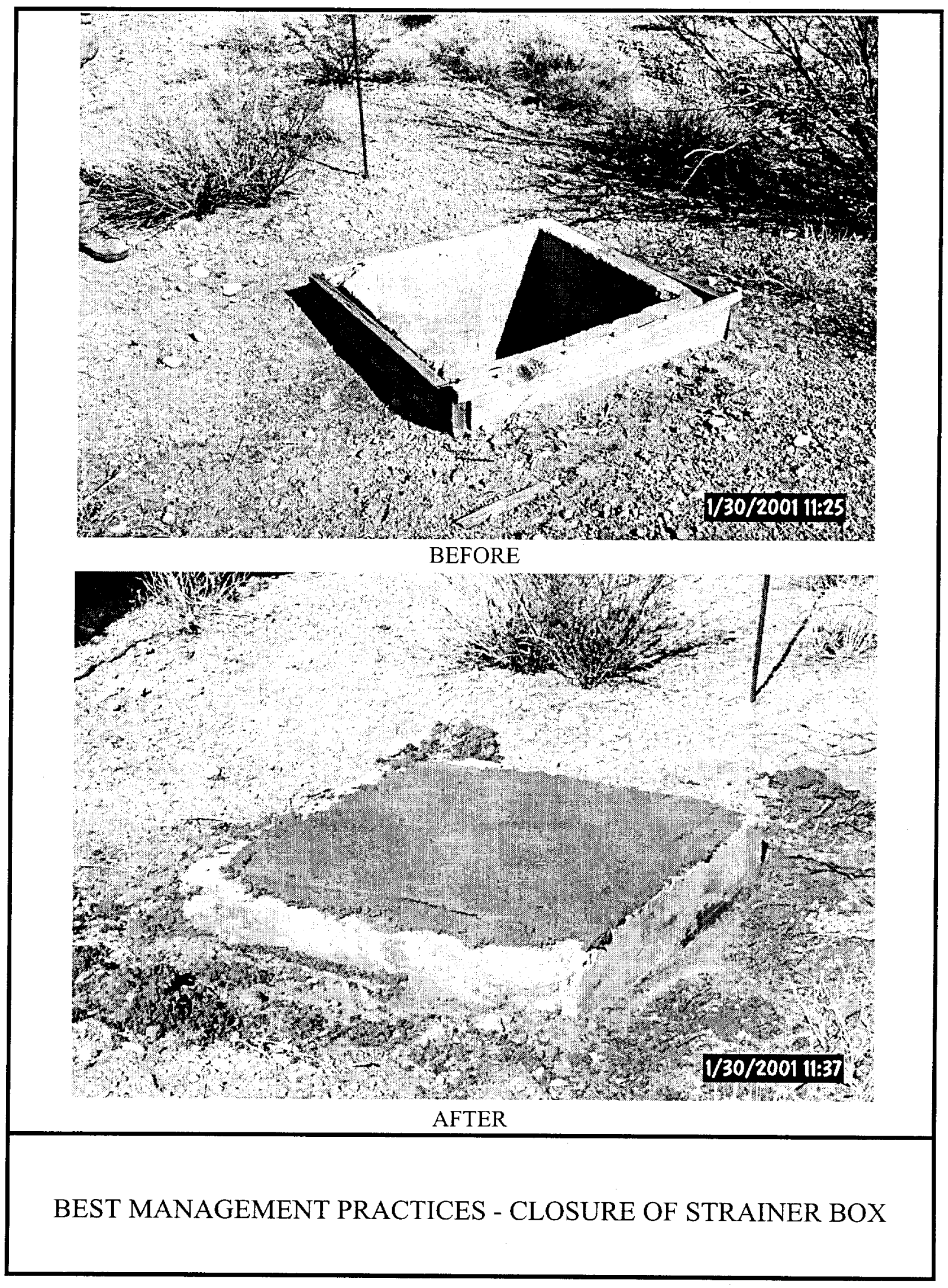




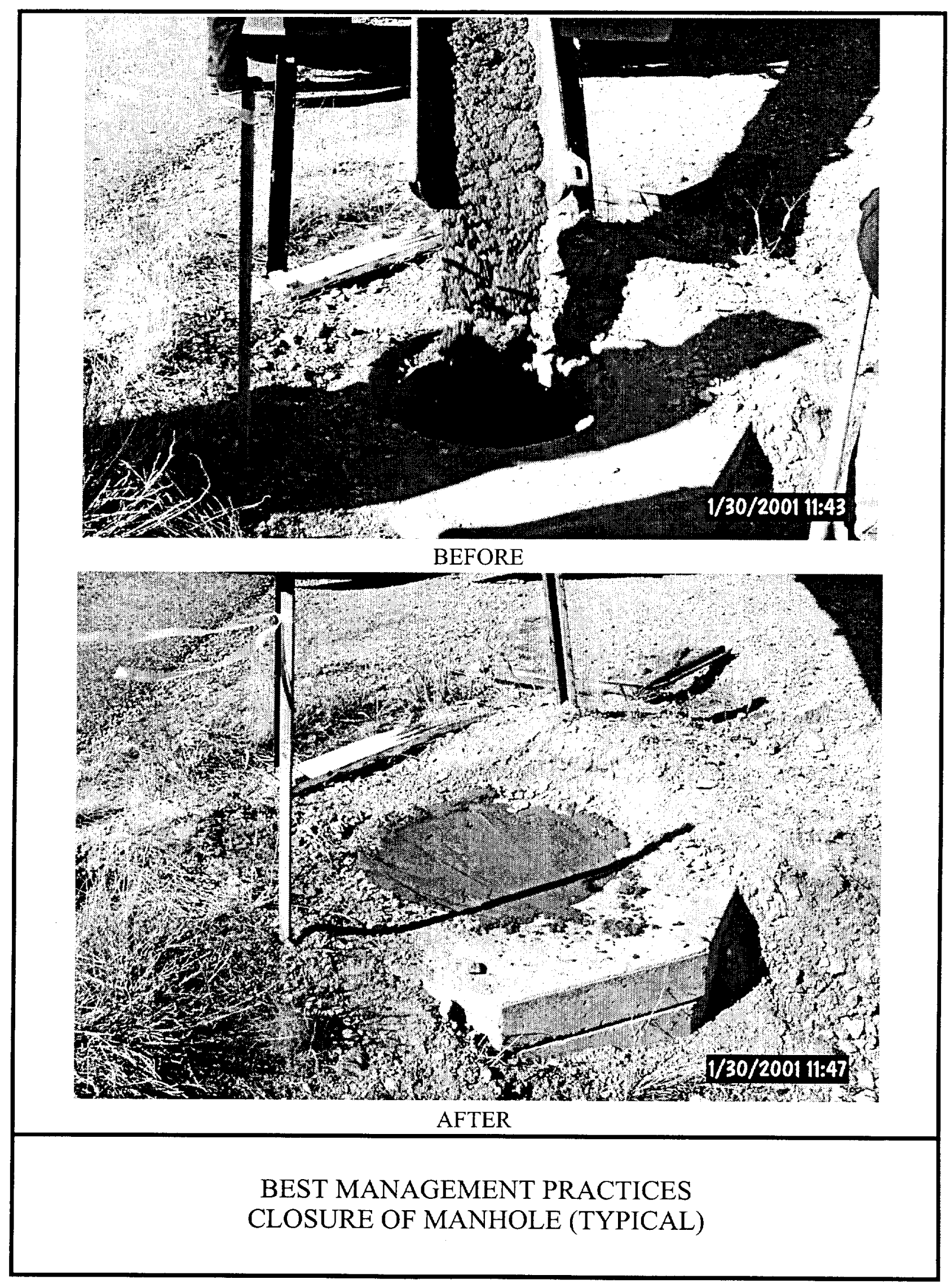




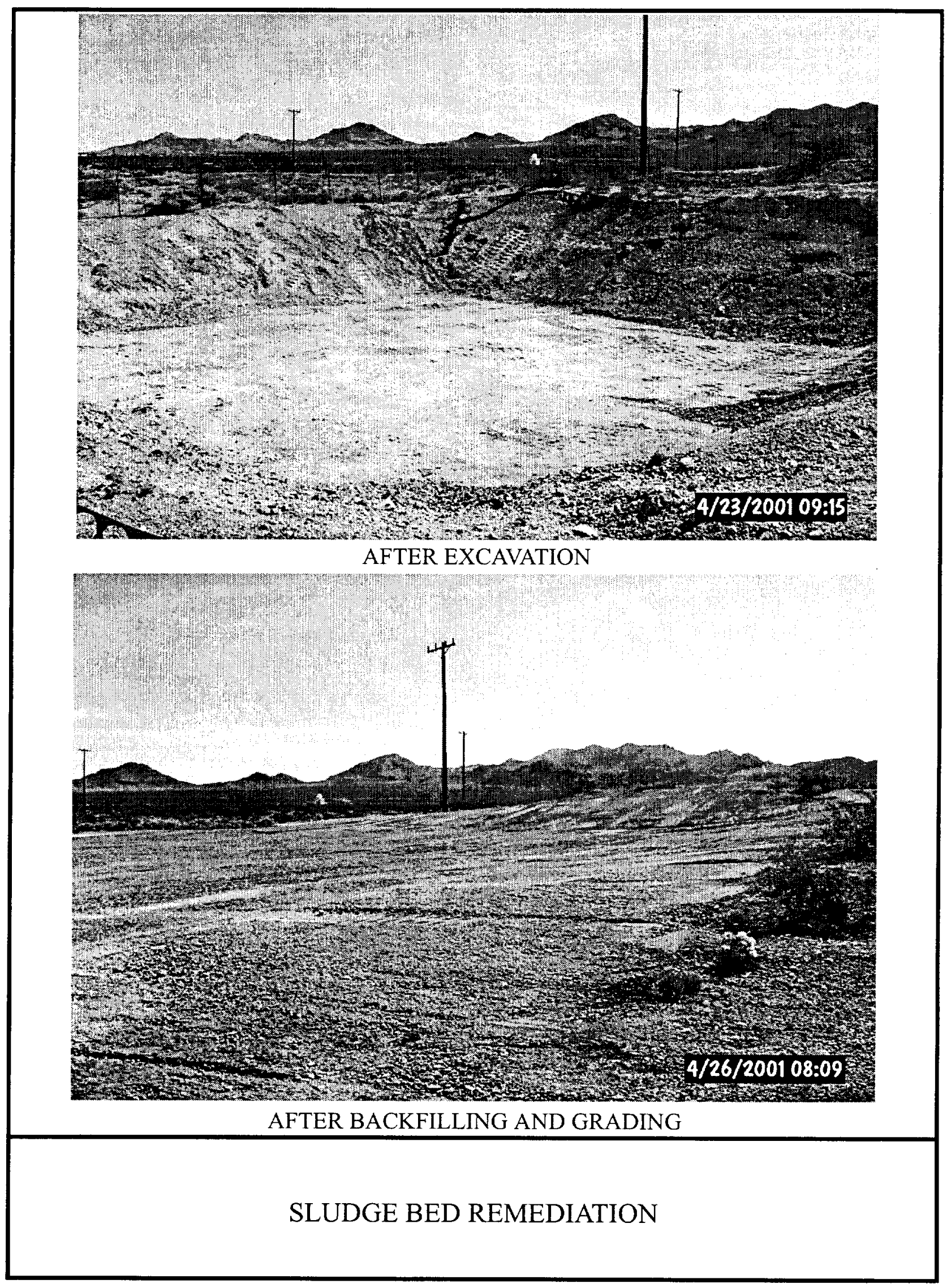


APPENDIX B

RECORDS OF TECHNICAL CHANGE 
THIS PAGE INTENTIONALLY LEFT BLANK 


\section{RECORD OF TECHNICAL CHANGE}

Technical Change No.

Project/Job No.

ProjectJJob Name

WBS 10401030419

CAU 230/320. Area 22 Sewage Lagoons and DRA Strainer Box
Page 1 of 1

Date 1/30/01

The following technical changes (including justification) are requested by:

Dennis Gustafson

(Name)
Task Manager

(Title)

The project time will be Unchanged).

Applicable Project-Specific Document(s): Corrective Action Plan for Corrective Action Unit 230/320, Area 22 Sewage Lagoons and DRA Strainer Box, Nevada Test Site, Nevada

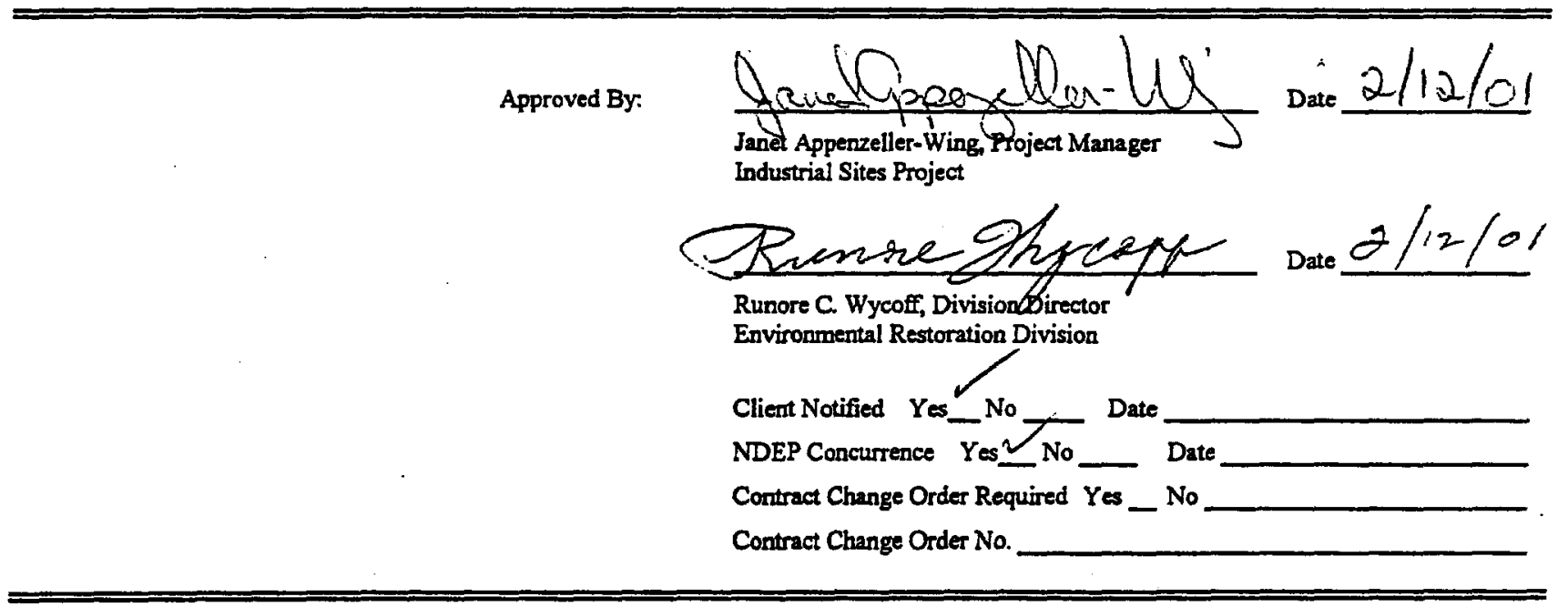


Site Characterization results from the sludge-bed area reported three samples having total petroleum hydrocarbons (TPH) as diesel-range organics at concentrations ranging from 150 milligrams per kilogram to 580 milligrams per kilogram. Excavation of the sludge-bed area has resulted in removal of construction debris and asphalt. During excavation activities, no soil staining, odor, or discoloration was observed. In addition, screening using a photoionization detector (PID) did not indicate the presence of any petroleum hydrocarbon discharge in this area.

For the purpose of verification sampling, the bottom and side slopes of the excavation will be divided into grids. The bottom of the excavation will be divided into 10 foot by 10 foot cells. The grid system will create 25 cells in the bottom of the excavation, each labeled using a grid coordinate system that will be created in the field. A random number generator (or table) will be used to select 5 of the cells. A surface soil sample will be collected from the center of each of the 5 cells and analyzed for TPH as diesel.

The side slopes of the excavation will be subdivided in plan view into 10 foot by 10 foot cells. The grid system will provide a total of 24 cells around the perimeter of the excavation, each labeled using a grid system that will be created in the field. A random number generator (or table) will be used to select 2 of the cells from each of the four side slopes. A surface soil sample will collected from the center of each of the 2 cells and analyzed for TPH as diesel. 


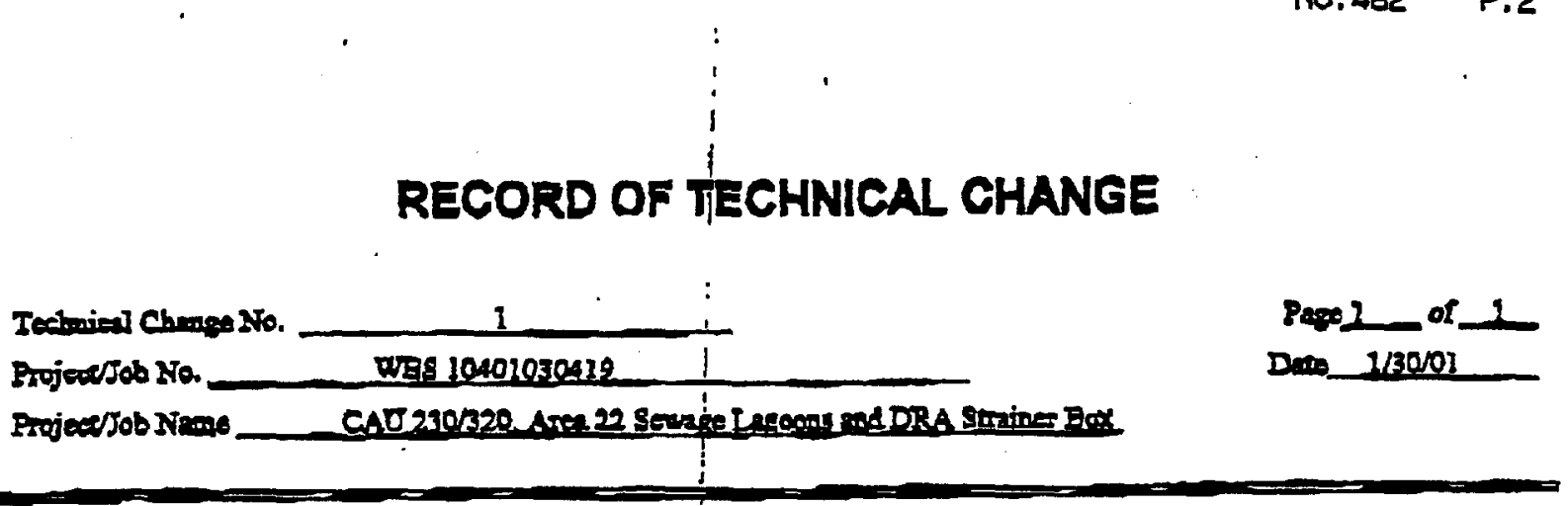

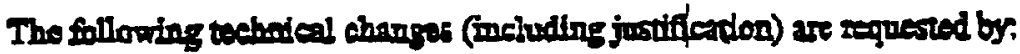
Denuir Gremfons

Tak Memarer

(Name)

(Tride)

The profect time will be Upehanged.

Applieble Preject-Speeifio Document(a): Corrective Action Ptan for Corrective Action Unit 230320, Area 22 Sewage Iagoons and DRA Stralner Box, Nevads Teot Site, Nevada

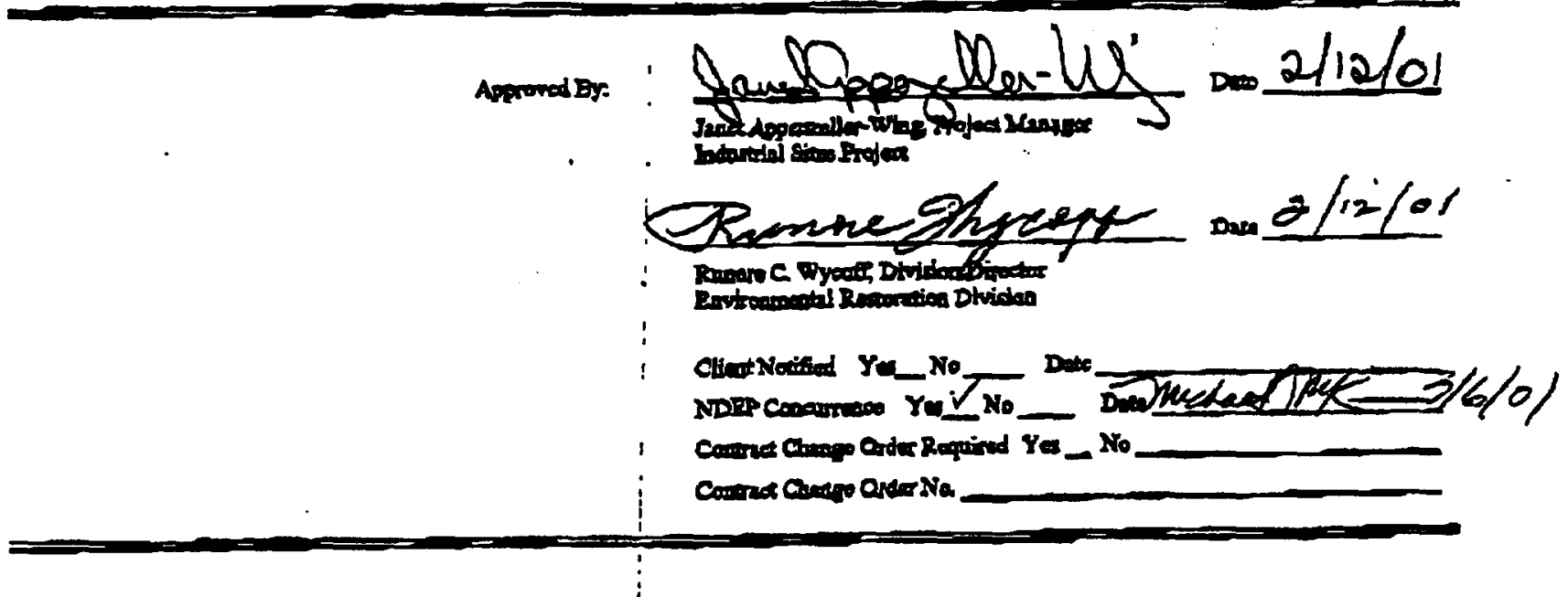




\section{RECORD OF TECHNICAL CHANGE}

Techrical Change No.

Project/Job No.

Project/Job Name
2

WBS 10401030419

CAU 230/320, Area 22 Sewage Lagoons and DRA Strainer Box
Page 1 of 2

Date $04 / 16 / 2001$

The following technical changes (including justification) are requested by:

Dennis Gustafson

(Name)
Task Manager

(Title)

The project time will be Unchanged)..

Applicable Project-Specific Document(s): Corrective Action Plan for Corrective Action Unit 230/320, Area 22

Sewage Lagoons and DRA Strainer Box, Nevada Test Site, Nevada

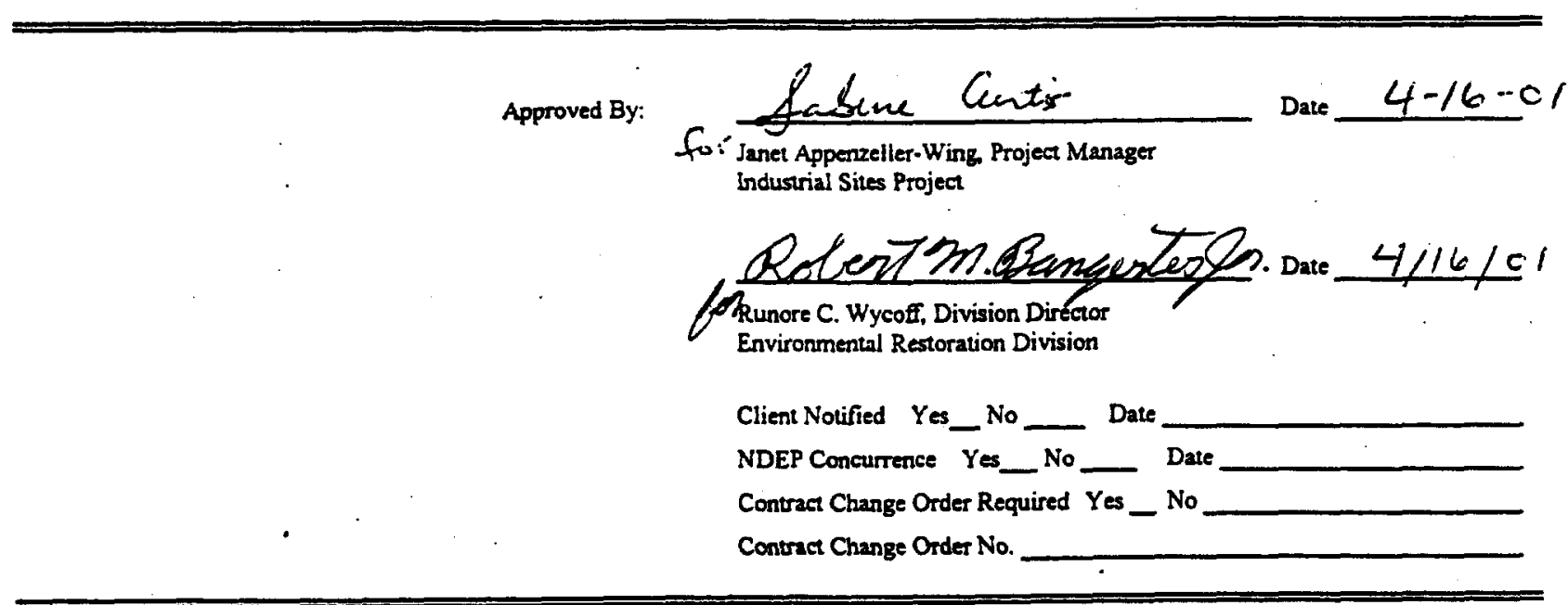




\section{RECORD OF TECHNICAL CHANGE}

Technieal Change No.

Project/Sob No.

2

Parfe 1 of 2

ProjectJob Name

WRS 10401030419

CAL 230/320 ATen 22 Eevare IArous and DRA Sirring Box

The following technical changes (including justification) ane requested by:

Dennis Grostafon

Task Manacer

(Name)

(Titile)

The project time will be Unchented).

Applicable Project-Specific Document(8): Corructive Aftion Plan for Corrective Aetion Unit 230/320, Area 22

Sewage Lagoons and DRA Striner Box, Nevada Tert Site, Nevada

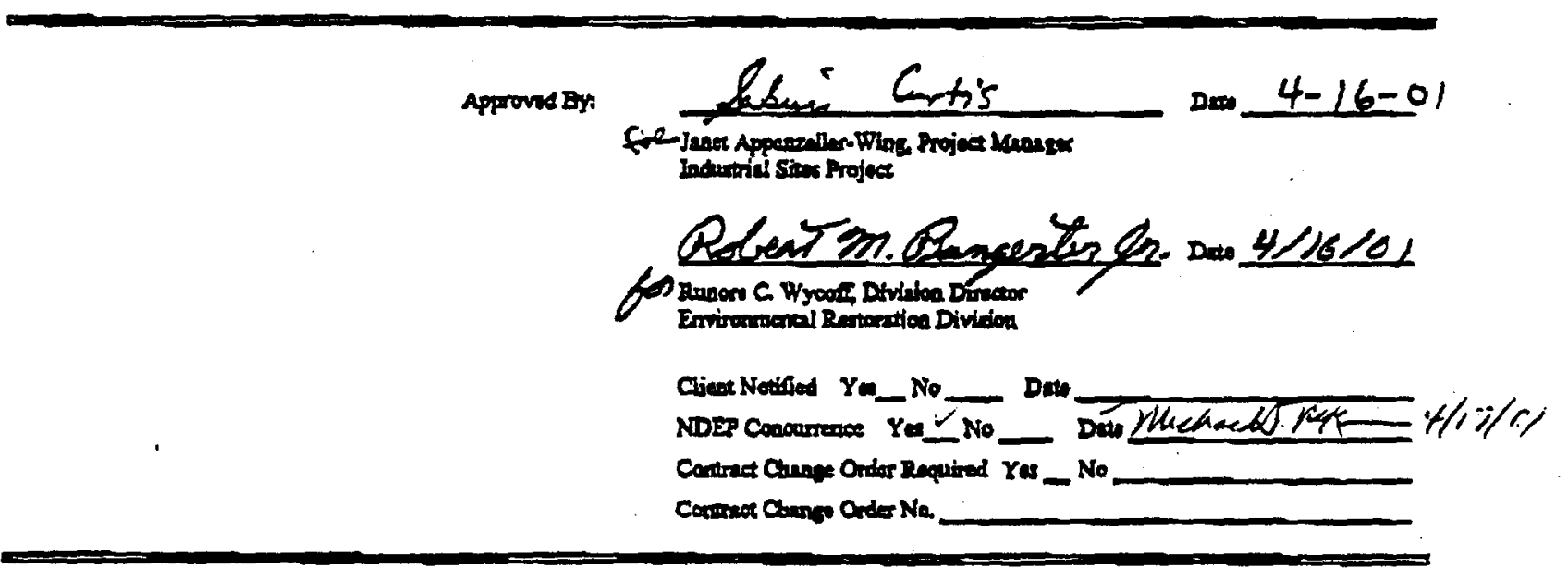


Technical Change No.

2

Page 2 of $\frac{2}{2}$

Project/Job No.

WBS 10401030419

Project/Job Name CAU 230/320, Area 22 Sewage Lagoons and DRA Strainer Box

Site characterization results from the sludge bed area reported three samples having total petroleum hydrocarbon (TPH) as diesel-range organics at concentrations ranging from 150 milligrams per kilogram $(\mathrm{mg} / \mathrm{kg})$ to $580 \mathrm{mg} / \mathrm{kg}$. Excavation of the sludge bed area has resulted in the removal of construction debris and asphalt. During excavation activities no soil staining, odor, or discoloration was identified. In addition, screening using a photoionization detector (PID) did not indicate the presence of any petroleum hydrocarbon discharge in this area.

Fifteen soil verification samples were collected on March 13, 2001. Laboratory analysis showed that 5 of the soil samples had concentrations of TPH as diesel range organics above the regulatory limit of 100 $\mathbf{m g} / \mathrm{kg}$.

Following an April 3, 2001 site visit with the Nevada Division of Environmental Protection, 13 additional soil verification samples were collected on April 9, 2001 to define the limits of the waste in the sludge bed area. Two of the samples were taken from the floor of the excavation. Eleven samples were taken higher on the sidewalls of the excavation than the original samples. Since the sidewalls are sloped this resulted in the samples being collected at a greater distance from the center of the excavated area.

Laboratory results showed that all 13 samples are below the MDL of $10 \mathrm{mg} / \mathrm{kg}$ for diesel range organics as petroleum hydrocarbons. Two asphalt samples were also collected and analyzed for TPH as diesel range organics. The chromatograms from the asphalt samples were compared with the chromatograms from the original verification soil samples collected on March 13, 2001 that had TPH as diesel range organics above the regulatory limits of $100 \mathrm{mg} / \mathrm{kg}$. The comparison of the chromatograms by an analytical chemist show that the TPH as diesel range organics seen in the soil verification samples is related to the asphalt that was disposed in the sludge bed area and not diesel. The sludge bed area will be backfilled with clean soil and the site will be clean closed with no administrative controls. 
- From: Sent:

To:

Cc:

Subject:
Gustafson, Dennis

Wednesday, April 04, 2001 3:14 PM

Curtis Sabine

Nacht, Steve; Johnson, Wayne; Appenzeller-Wing Janet

Sabine,

Per our phone conversation, myself, Steve Nacht, and Greg Raab from NDEP did a site visit this morning at CAU 230/320. Mr. Raab stated that he did not feel that the lateral extent of contamination has yet been determined. We talked about the small fragments of asphalt that is still present in the side walls and that we believe that the TPH that we are seeing in the verification samples is associated with this asphalt. Mr. Raab suggested that we perform additional soil sampling using the Petroflag field screening higher on the sidewalls which would be a greater distance from the current bottom of the excavation. He said that if these show less than the regulatory limit of $100 \mathrm{mg} / \mathrm{kg}$ then the extent of the waste has been defined and we would not be required to do additional excavation. He also requested documentation that asphalt contains diesel range organics similar to those that we have seen in the verification samples. We have identified an additional 12 sample locations. We will perform Petroflag analysis at each of the 12 locations and also send the samples to the lab for TPH diesel range organics analysis. I am not sure if the NDEP would call this a clean closure since we would provide supporting documentation that the constituent of concern is only asphalt or if it would be approved as a closure in place with use restrictions. You may want to discuss the above scope with the NDEP and get there final concurrence before we sample and also clarify if this would be a clean closure or not.

If you concur with this path forward please let me know and we will perform the sampling tomorrow. If you have any questions please contact Steve Nacht at 5-7234 since I will be out of the office until Monday or I can be reached by pager at 794-6497.

Thanks, Dennis 
THIS PAGE INTENTIONALLY LEFT BLANK 
APPENDIX C

WASTE DISPOSITION DOCUMENTATION 
THIS PAGE INTENTIONALLY LEFT BLANK 
Date of Receipt

Type of Waste
Weight in

Pounds
Origin of Waste Area No. Building No.

REA 23

$$
\text { 21-MAR-2001 NTS } 5,800 \quad 23.190
$$

Comments: Plastic, wood, paper, soil, rocks, cement and concrete.

\begin{tabular}{lllll}
\hline 21-MAR-2001 PUTRESCIBLE & 780 & 23 & \&CP CAF
\end{tabular}

Comments:

Total for AREA 23

6,580 Pounds

3.29 Tons

REA 6

21-MAR-2001 NTS

8,000

06

$6-800$

Comments: Soil.

\section{Total for AREA 6}

\section{8,000 Pounds}

4 Tons

...REA 9

21-MAR-2001 NTS

12,800

03

$3 C-26$

Comments: Mfg.items, demolition debris.

21-MAR-2001 FFACO-ONSITE $8,180 \quad 22$ CAU

Comments: CAU 230/320 - Asphalt, plastic, metal, wire, wood, cable, cement and conctete.

21-MAR-2001 NTS

$16,500 \quad 03 \quad 3 C-45$

Comments: Mfg.items, demolition debris.

21-MAR-2001 NTS . $14,600 \quad 03 \quad 3$ C-45

Comments: Mfg.items, demolition debris.

\begin{tabular}{lllll}
\hline $21-M A R-2001$ & NTS & 24,900 & 03 & $3 C-45$
\end{tabular}

Comments: Mfg.items, demolition debris.

\begin{tabular}{lllll}
\hline $21-M A R-2001$ & NTS & 19,200 & 03 & $3 C-45$
\end{tabular}

Comments: Mfg.items, demolition debris.

\begin{tabular}{lllll}
\hline $21-M A R-2001$ & NTS & 23,260 & 03 & $3 C-45$
\end{tabular}

Comments: Mfg.items, demolition debris.

\begin{tabular}{lllll}
\hline $21-M A R-2001$ & NTS & 32,000 & 03 & $3 C-45$
\end{tabular}

Comments: Mfg.items, demolition debris.

\begin{tabular}{lllll}
\hline $21-M A R-2001$ & NTS & 21,330 & 03 & $3 C-45$
\end{tabular}

Comments: Mfg.items, demolition debris.

\begin{tabular}{lllll}
\hline $21-M A R-2001$ & NTS & 19,630 & 03 & $3 C-45$
\end{tabular}

Comments: Mfg.items, demolition debris. 


\section{Bechtel Nevada}

DATE: $327 \%$ OI

LANDFILL DAILY ACCESS REGISTER

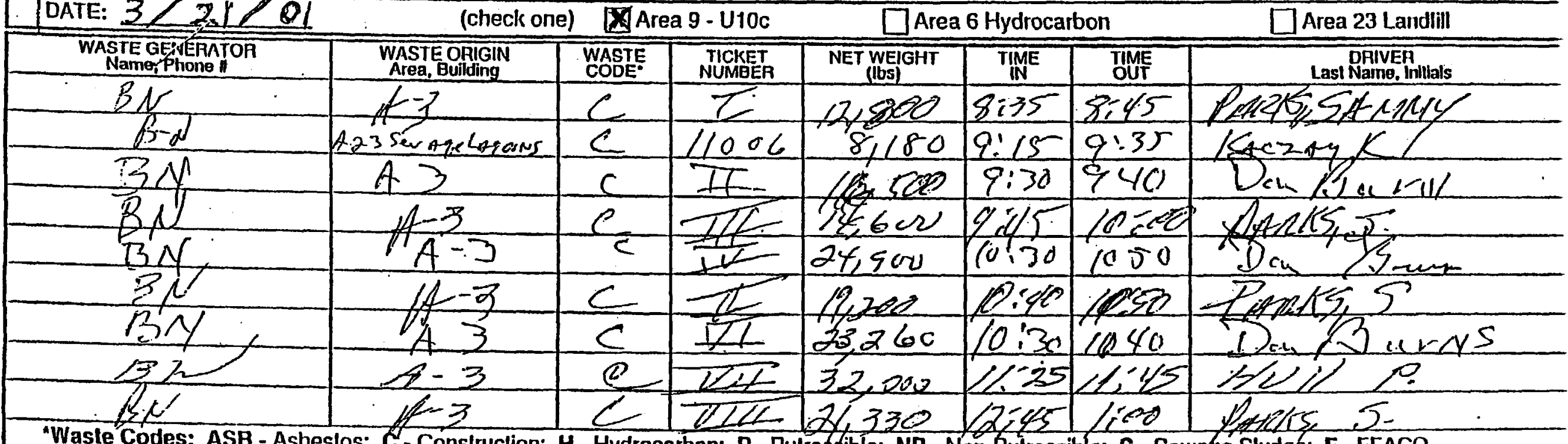

Waste Codes: ASB - Asbeslos; C-Construclion; H - Hydrocarbon; P - Putrescible; NP - Non-Pulrescible; S - Sewage Sludge; F - FFACO

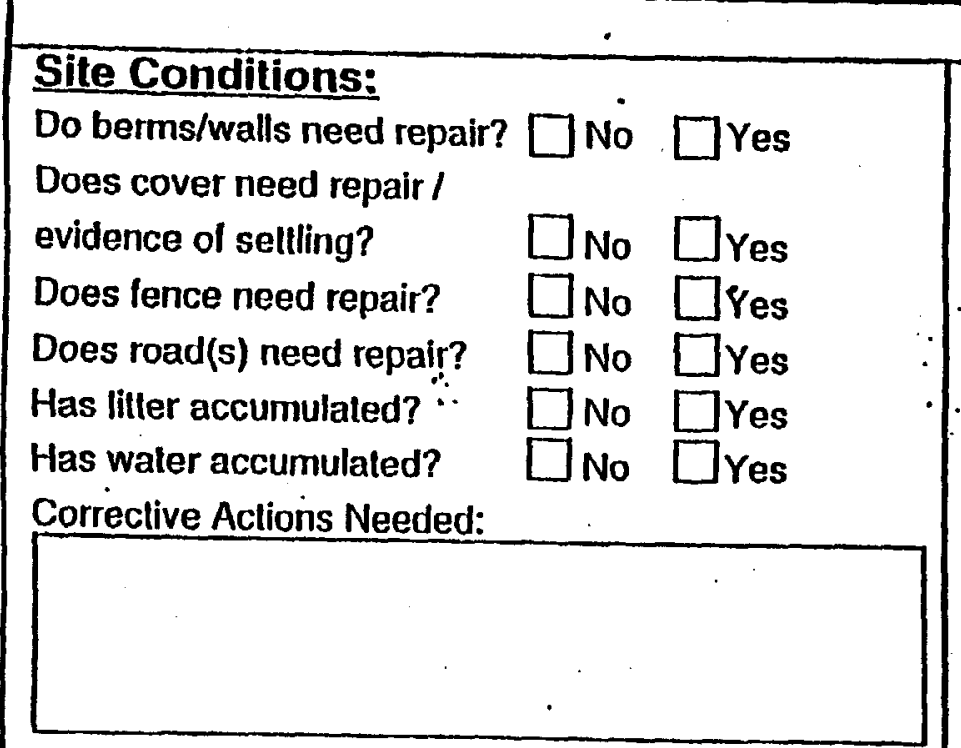

Corrective Actions Taken: (description, name, dale):

\section{INSPECTION INFORMATION}

\section{Random Load Inspection:}

$\square$ No prohibiled waste was found

$\square$ Yes, the prohibiled waste(s) identilied below were lound.

$\square$ Putrescible waste (prohibited in U10c and Area 6 Landlills).

$\square$ Hazardous wasle per NAC 444.580

$\square$ PCB wasle regulated by TSCA

$\square$ Wasle containing free liquids

$\square$ TSCA-regulated

$\square$ Waste failing the "no added radioactivity" per the POC requirement.

$\square$ Friable asbestos (prohibited in U1Oc and Area 6 Landfills)

$\square$ Hydrocarbon soil at $>100 \mathrm{ppm}$ TPH (prohibited in 23, allowed in U10c provided less than 50 cubic yards/week are disposed)

Corrective Aclions Taken: (description, name, date, who notified):

\section{NSPECTED BY}

(date/time): 


\begin{tabular}{cccc}
$\begin{array}{c}\text { Date of } \\
\text { Receipt }\end{array}$ & $\begin{array}{c}\text { Type of } \\
\text { Waste }\end{array}$ & $\begin{array}{c}\text { Weight in } \\
\text { Pounds }\end{array}$ & $\begin{array}{c}\text { Origin of Waste } \\
\text { Area No. Building No. }\end{array}$ \\
\hline
\end{tabular}

Indfill Id

24-JAN-2001 PUTRESCIBLE

$360 \quad 06$

CAFE

Comments:

24-JAN-2001 NON-PUTRESCIBLE 21,360 NTS ALL

Comments:

\begin{tabular}{lllll}
\hline $23-J A N-2001$ & PUTRESCIBLE & 620 & 23 & \&CP CAF
\end{tabular}

Comments:

Total for AREA 23

12-FEB-2001 FFACO-ONSITE
89,060 Pounds

44.53 Tons

Comments: Hydrocarbon soil, asphalt, wire, metal, CAU 230/320.

12-FEB-2001 FFACO-ONSITE $54,980.22$ CAU'S

Comments: Hydrocarbon soil, asphalt, metal, wire, CAU 230/320.

12-FEB-2001 FFACO-ONSITE $63,940 \quad 22$ CAU'S

Comments: Hydrocarbon soil, asphalt, metal, wire, CAU 230/320.

12-FEB-2001 FFACO-ONSITE 52,940 22 CAU'S

Comments: Hydrocarbon soil, asphalt, metal, wire, CAU 230/320.

12-FEB-2001 FFACO-ONSITE $53,420 \quad 22$ CAU'S

Comments: Hydrocarbon soil, asphalt, wire, metal, CAU $230 / 320$.

\begin{tabular}{lllll}
\hline $12-F E B-2001$ & . FFACO-ONSITE & 59,660 & 22 & CAU'S
\end{tabular}

Comments: Hydrocarbon soil, asphalt, wire, metal, CAU 230/320.

12-FEB-2001 FFACO-ONSITE $57,480 \quad 22 \quad$ CAU'S

Comments: Hydrocarbon soil, asphalt, wire, metal, CAU 230/320

08-FEB-2001 FFACO-ONSITE $52,040 \quad 22 \quad$ CAU'S

Comments: Hydrocarbon soil, asphalt, metal, wire, CAU 230/320.

08-FEB-2001 FFACO-ONSITE $53,060 \quad 22$ CAU'S

Comments: Hydrocarbon soil, asphalt, wire, metal, CAU 230/320.

O8-FEB-2001 FFACO-ONSITE $59,260 \quad 22 \quad$ CAU'S

Comments: Hydrocarbon soil, asphalt, wire, metal, CAU $230 / 320$.

\begin{tabular}{lllll}
\hline 08-FEB-200i FFACO-ONSITE & 53,800 & 22 & CAU'S
\end{tabular}

Comments: Hydrocarbon soil, asphalt, wire, metal, CAU 230/320. 
Type of Waste
Weight in Pounds
Origin of Waste Area No. Building No.

AREA 6

08-FEB-2001 FFACO-ONSITE

53,660

22

CAU'S

Comments: Hydrocarbon soil, asphalt, metal, wire, CAU 230/320.

08-FEB-2001 FFACO-ONSITE $54,800 \quad 22$ CAU'S

Comments: Hydrocarbon soil, asphalt, wire, metal, CAU 230/320.

08-FEB-2001 FFACO-ONSITE $52,600 \quad 22$ CAU'S

Comments: Hydrocarbon soil, asphalt, wire, metal, CAU $230 / 320$.

06-FEB-2001 FFACO-ONSITE $61,480 \quad 22$ CAU'S

Comments: Hydrocarbon soil, asphalt, metal, wire, CAU 230/320

06-FEB-2001 FFACO-ONSITE $45,180 \quad 22$ CAU'S

Comments: Hydrocarbon soil, asphalt, wire, metal, CAU 230/320.

06-FEB-2001 FFACO-ONSITE $51,460 \quad 22$ CAU'S

Comments: Hydrocarbon soil, asphalt, wire, metal, CAU $230 / 320$.

06-FEB-2001 FFACO-ONSITE $47,580 \quad 22$ CAU'S

Comments: Hydrocarbon soil, asphalt, wire, metal, CAU 230/320.

06-FEB-2001 FFACO-ONSITE $59,920 \quad 22$ CAU'S

Comments: Hydrocarbon soil, asphalt, wire, metal, CAU 230/320.

06-FEB-2001 FFACO-ONSITE $57,140 \quad 22 \quad$ CAU'S

Comments: Hydrocarbon soil, asphalt, wire, metal, CAU 230/320.

06-FEB-2001 FFACO-ONSITE $59,060 \quad 22$ CAU'S

Comments: Hydrocarbon soil, asphalt, wire, metal, CAU 230/320.

06-FEB-2001 FFACO-ONSITE $\quad 67,260 \quad 22$ CAU'S

Comments: Hydrocarbon soil, asphalt, metal, wire, CAU 230/320.

\begin{tabular}{lllll}
\hline $31-J A N-2001$ & FFACO-ONSITE & 64,120 & 22 & CAU'S
\end{tabular}

Comments: Hydrocarbon soil, asphalt, metal, wire, CAU 230/320.

31-JAN-2001 FFACO-ONSITE $55,400 \quad 22$ CAU'S

Comments: Hydrocarbon soil, asphalt, wire, metal, CAU 230/320.

31-JAN-2001 FFACO-ONSITE $56,320 \quad 22 \quad$ CAU'S

Comments: Hydrocarbon soil, asphalt, wire, metal, CAU 230/320.

31-JAN-2001 FFACO-ONSITE $57,660 \quad 22$ CAU'S

Comments: Hydrocarbon soil, asphait, wire, metal, CAU 230/320.

\begin{tabular}{lllll}
\hline $31-J A N-2001$ & FFACO-ONSITE & 55,480 & 22 & CAU'S
\end{tabular}

Comments: Hydrocarbon soil, asphalt, metal, wire, CAU 230/320. 
Solid Waste Operations

Solid Waste Received Between 23-JAN-2001 and 13-FEB-2001

Sorted by Landfill ID, Receipt Date

F pared on 27-MAR-2001

Origin of Waste Area No. Building No.

REA 6

$$
\text { 31-JAN-2001 FFACO-ONSITE }
$$

62,020

22

CAU'S

Comments: Hydrocarbon soil, asphalt, wire, metal, CAU 230/320.

\begin{tabular}{lllll}
\hline $31-J A N-2001$ & FFACO-ONSITE & 61,800 & 22 & CAU'S
\end{tabular}

Comments: Hydrocarbon soil, asphalt, wire, metal, CAU $230 / 320$.

31-JAN-2001 FFACO-ONSITE $\quad 55,920 \quad 22$ CAU'S

Comments: Hydrocarbon soil, asphalt, metal, wire, CAU 230/320.

$\begin{array}{lllll}31-J A N-2001 & \text { FFACO-ONSITE } & 56,980 & 22 & \text { CAU'S }\end{array}$

Comments: Hydrocarbon soil, asphalt, metal, wire, CAU 230/320.

30-JAN-2001 FFACO-ONSITE $56,760 \quad 22 \quad$ CAU'S

Comments: Hydrocarbon soil, asphalt, metal, wire, CAU $230 / 320$.

30-JAN-2001 FFACO-ONSITE $66,260 \quad 22 \quad$ CAU'S

Comments: Hydrocarbon soil, asphalt, metal, wire, CAU 230/320.

\begin{tabular}{lllll}
\hline $30-J A N-2001$ & FFACO-ONSITE & 57,800 & 22 & CAU'S
\end{tabular}

Comments: Hydrocarbon soil, asphalt, metal, wire, CAU $230 / 320$.

\begin{tabular}{lllll}
\hline $30-J A N-2001$ & FFACO-ONSITE & 65,920 & 22 & CAU'S
\end{tabular}

Comments: Hydrocarbon soil, asphalt, metal, wire, CAU 230/320.

30-JAN-2001 FFACO-ONSITE $56,880 \quad 22$ CAU'S

Comments: Hydrocarbon soil, asphalt, wire, metal, CAU 230/320.

30-JAN-2001 FFACO-ONSITE $62,380 \quad 22$ CAU'S

Comments: Hydrocarbon soil, asphalt, wire, metal, CAU 230/320.

\begin{tabular}{lllll}
\hline $30-J A N-2001$ & FFACO-ONSITE & 61,660 & 22 & CAU'S
\end{tabular}

Comments: Hydrocarbon soil, asphalt, wire, metal, CAU 230/320.

30-JAN-2001 FFACO-ONSITE $56,820 \quad 22$ CAU'S

Comments: Hydrocarbon soil, asphalt, metal, wire, CAU 230/320.

30-JAN-2001 FFACO-ONSITE $56,680 \quad 22$ CAU'S

Comments: Hydrocarbon soil, asphalt, wire, metal, CAU 230/320.

30-JAN-2001 FFACO-ONSITE $55,760 \quad 22 \quad$ CAU'S

Comments: Hydrocarbon soil, asphalt, metal, wire, CAU 230/320.

30-JAN-2001 FFACO-ONSITE $66,620 \quad 22 \quad$ CAU'S

Comments: Hydrocarbon soil, asphalt, metal, wire, CAU 230/320.

30-JAN-2001 FFACO-ONSITE $\quad 59,580 \quad 22$ CAU'S

Comments: Hydrocarbon soil, asphalt, wire, metal, CAU 230/320. 


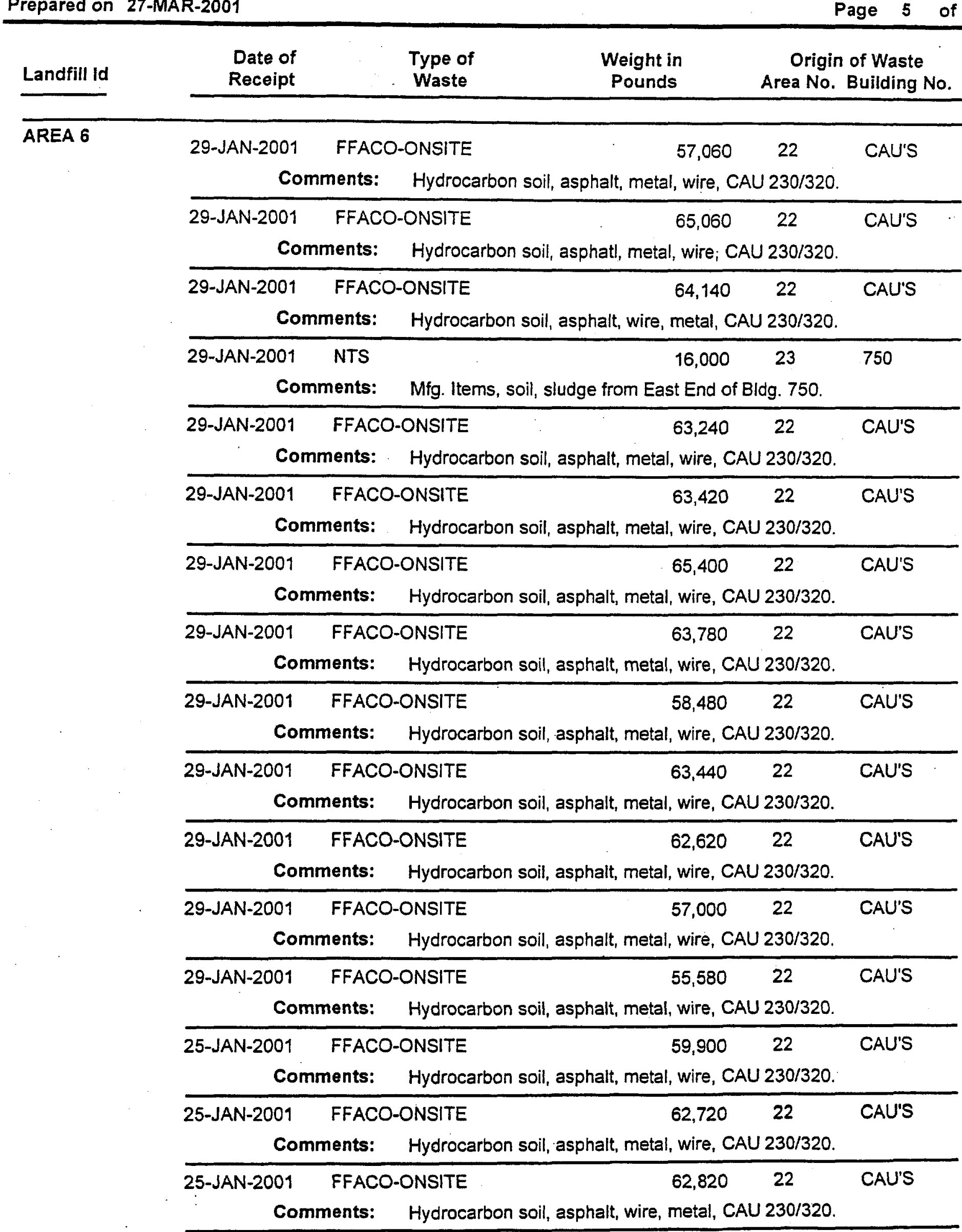


Comments: Hydrocarbon soil, asphalt, wire, metal, CAU 230/320.

25-JAN-2001 FFACO-ONSITE $66,700 \quad 22$ CAU'S

Comments: Hydrocarbon soil, asphalt, wire, metal, CAU 230/320.

25-JAN-2001 FFACO-ONSITE $64,280 \quad 22$ CAU'S

Comments: Hydrocarbon soil, asphalt, wire, metal, CAU $230 / 320$.

25-JAN-2001 FFACO-ONSITE $63,400 \quad 22$ CAU'S

Comments: Hydrocarbon soil, asphalt, metal, wire, CAU 230/320.

25-JAN-2001 FFACO-ONSITE $55,560 \quad 22$ CAU'S

Comments: Hydrocarbon soil, asphalt, metal, wire, CAU 230/320.

\begin{tabular}{lllll}
\hline 25-JAN-2001 FFACO-ONSITE & 64,860 & 22 & CAU'S
\end{tabular}

Comments: Hydrocarbon soil, asphalt, metal, wire, CAU $230 / 320$.

25-JAN-2001 FFACO-ONSITE $61,560 \quad 22$ CAU'S

Comments: Hydrocarbon soil, asphalt, metal, wire, CAU $230 / 320$.

\begin{tabular}{lllll}
\hline $25-J A N-2001$ & FFACO-ONSITE & 60,980 & 22 & CAU'S
\end{tabular}

Comments: Hydrocarbon soil, asphalt, wire, metal, CAU 230/320.

\begin{tabular}{lllll}
\hline $25-J A N-2001$ & FFACO-ONSITE & 63,500 & 22 & CAU'S
\end{tabular}

Comments: Hydrocarbon soil, asphalt, metal, wire, CAU $230 / 320$.

\begin{tabular}{lllll}
\hline $24-J A N-2001$ & NTS & 58,880 & 22 & CAU'S
\end{tabular}

Comments: Hydrocarbons, asphalt, metal, wire. CAU 230/320.

\begin{tabular}{llrrr}
\hline 24-JAN-2001 NTS & 66,300 & 22 & CAU'S
\end{tabular}

Comments: Hydrocarbons, asphalt, metal, wire. CAU 230/320.

24-JAN-2001 NTS $60,320 \quad 22 \cdot$ CAU'S

Comments: Hydrocarbons, asphalt, metal, wire. CAU 230/320.

24-JAN-2001 NTS $66,440 \quad 22$ CAU'S

Comments: Hydrocarbons, asphalt, metal, wire. CAU 230/320.

Comments: Wood, woil, cement and concrete.

\begin{tabular}{ccccc}
\hline 05-FEB-2001 NTS & 22,000 & 25 & RMAD \\
Comments: Non-asbestosform insulation. & & & \\
\hline
\end{tabular}




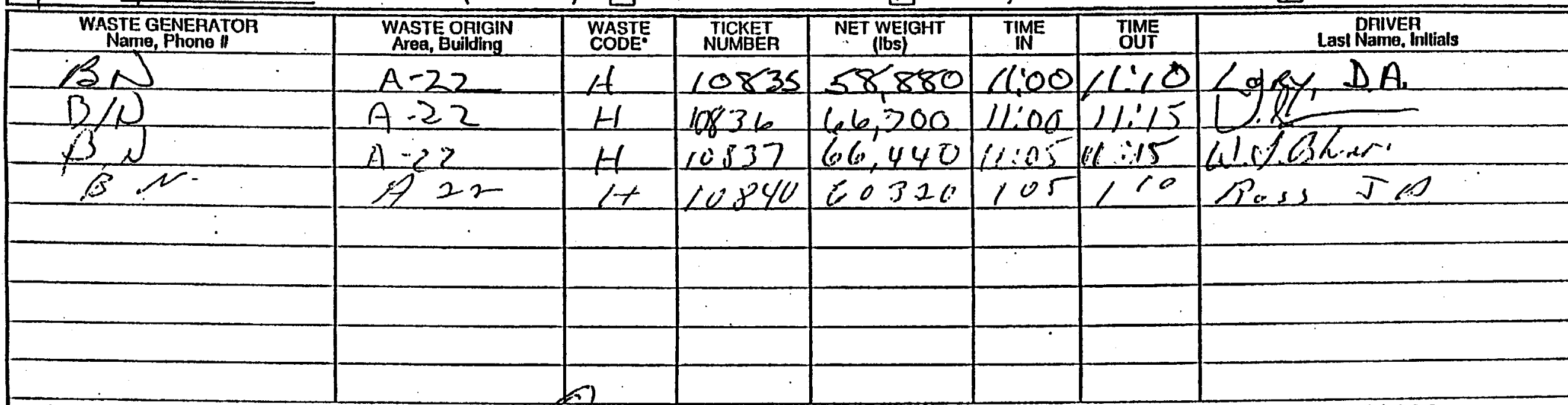

"Waste Codes: ASB - Asbestos; C - Construclion H H-Hydrocarbon; P - Pulrescible; NP - Non-Putrescible; S - Sewage Sludge; F- FFACO

\section{Site Conditions:}

Do berms/walls need repair? $\square$ No: $\square$ Yes

Does cover need repair / evidence of setlling?

Does fence need repair?

Does road(s) need repair? Has litter accumulaled? $\because$

Has water accumulaled?

Corrective Aclions Needed:

$\square_{\text {No }} \square_{\text {Yes }}$
$\square_{\text {No }} \square_{\text {Yes }}$
$\square_{\text {No }} \square_{\text {Yes }}$
$\square_{\text {No }}$ पYes

INSPECTION INFOAMATION

\section{Rardom Load Inspection:}

$\square$ No prohibiled waste was found

$\square$ Yes, the prohibited wasle(s) idenlified below were found.

$\square$ Putrescible wasle (prohibiled in U10c and Area 6 Landfills).

$\square$ Hazardous wasle per NAC $\mathbf{4 4 4 . 5 8 0}$

$\square$ PCB waste regulated by TSCA

$\square$ Wasle containing free liquids

$\square$ TSCA-regulated

$\square$ Wasle lailing the "no added radioaclivily" per the POC requirement.

$\square$ Friable asbestos (prohibiled in U1Oc and Area 6 Landiils)

$\square$ Hydrocarbon soil al >100 ppm TPH (prohibited in 23, allowed in U10c provided less than 50 cubic yards/week are disposed)

Corrective Actions Taken: (descriplion, name, date, who notilied):

Corrective Actions Taken: (description, name, dale): iveter on cuent side $50^{\prime} \times 50^{\prime} \times 2{ }^{\prime \prime}$

\section{INSPECTED BY}

(date/time):

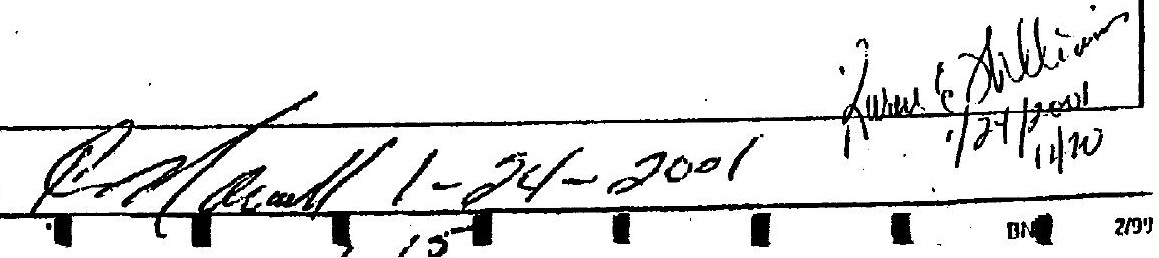




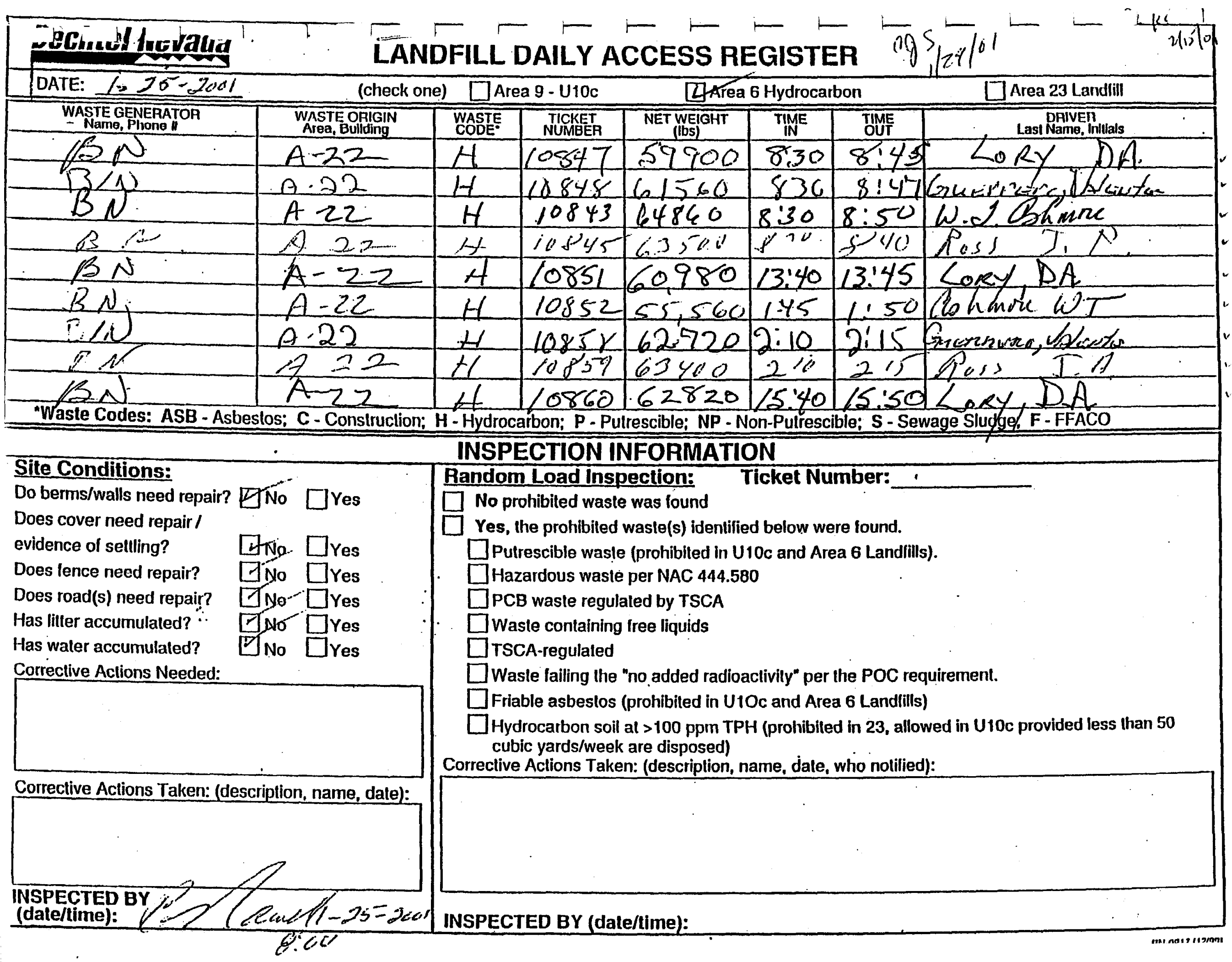


LANDFILL DAILY ACCESS REGISTER $C Q{ }^{3}, 1 / 2 \% 1$

DATE: 1250

(check one) $\square$ Area 9-U10c : [B] Area 6 Hydrocarbon

$\square$ Area 23 Landlill

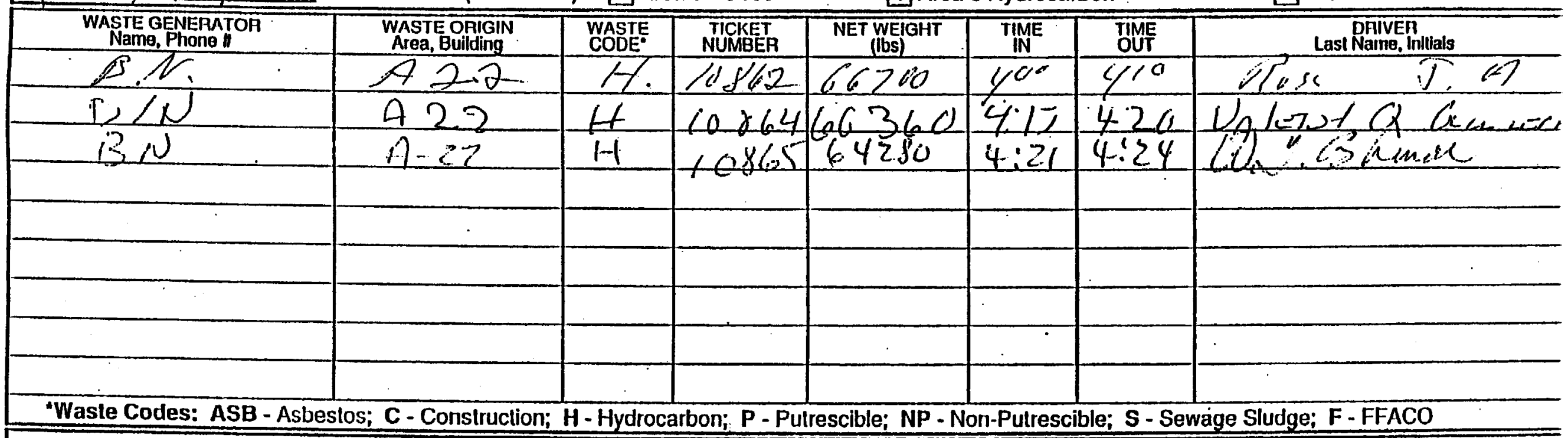

\section{Site Conditions:}

Do berms/walls need repair? $\square$ No $\square$ Yes Does cover need repair/ evidence of sellling? Does lence need repair? Does road(s) need repair? Has litter accumulated? $\because$ Has water accumulated? Corrective Aclions Needed: 刓No $\square$ Yes $\square$ No $\square$ Yes $\square$ No $\square$ res $\square$ No $\square$ Yes [No $\square$ Yes

Corrective Aclions Taken: (description, name, date):

\section{INSPECTION INFOAMATION}

\section{Fandom Load Inspection:}

$\square$ No prohibiled waste was found

$\square$ Yes, the prohibiled waste(s) Identified below were found.

$\square$ Putrescible wasle (prohibiled in U10c and Area 6 Landfilis).

$\square$ Hazardous waste per NAC 444.580

$\square$ PCB waste regulated by TSCA

$\square$ Waste containing free liquids

$\square$ TSCA-regulated

$\square$ Waste lailing the "no added radioactivity" per the POC requirement.

$\square$ Friable asbestos (prohibiled in U1Oc and Area 6 Landifils)

$\square$ Hydrocarbon soil al >100 ppm TPH (prohibiled in 23, allowed in U10c provided less than 50 cubic yards/week are disposed)

Corrective Actions Taken: (description, name, date, who notilied):
INSPECTED BY (dateltime): 


\section{PEinal hevalug}

LANDFILL DAILY ACCESS REGISTER $(\sqrt{1 / 5} / 30 / 01$

DATE: $1-29-2001$

(check one) 口Area 9 - U10c

DArea 6 Hydrocarbon

$\square$ Area 23 Landlill

WASTEGENERTOA

$13+5$

$3 / 1$

?

$\left|\begin{array}{l}\text { WASTEORIGIN } \\ \text { Area, Building } \\ A 22 \\ A-22 \\ A-22 \\ A 22 \\ A .23 / 750 \\ A-22 \\ A-22 \\ A-22 \\ A-22\end{array}\right|$

\begin{tabular}{|c|c|c|}
\hline $\begin{array}{l}\text { WASTE } \\
\text { CODE }\end{array}$ & $\begin{array}{l}\text { TICKET } \\
\text { NUMBER }\end{array}$ & $\begin{array}{l}\text { NET WEIGHT } \\
\text { (bs) }\end{array}$ \\
\hline it & 16196 & 57060 \\
\hline 4 & 10860 & 55580 \\
\hline$H$ & 16869 & 57000 \\
\hline $1 t$ & 1087 & 65060 \\
\hline$y$ & $\mathbb{Z}$ & $1,6,000$ \\
\hline$\theta$ & 10874 & 64140 \\
\hline H & 10.875 & 62620 \\
\hline $\mathrm{H}$ & $10 \times 76$ & 6.3440 \\
\hline Fr & 10007 & .0480 \\
\hline
\end{tabular}

$\frac{T M E}{\text { TME }}$
$2 ! 35$
$9 ! 35$
940
$10^{00}$
$10^{70}$
1135
$11: 45$
$11: 45$
$11^{50}$

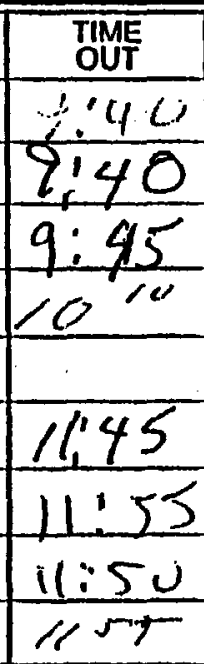
Lasi Name. Inlfials

"Waste Codes: ASB - Asbestos; C - Construclion; H - Hydrocarbon; P - Pulrescible; NP - Non-Pulrescible; S - Sewage Sludge; F - FFACO

\section{Site Conditions:}

Do berms/walls need repair? $\square$ No $\square$ Yes Does cover need repair /

evidence of sellling?

Does lence need repair?

Does road(s) need repair?

Has litter accumulaled? $\because$

Has water accumulated?

Corrective Aclions Needed:

$\square$ No $\square$ Yes

$\square$ No $\square$ res

$\square$ No $\square$ res

$\square$ No $\square$ res

$\square$ No $\square$ Yes

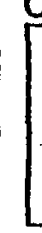

Corrective Actions Taken: (description, name, date):

\section{INSPECTED BY}

(date/time):

\section{INSPECTION INFORMATION}

\section{Random Load Inspection: Ticket Number: .}

$\square$ No prohibiled wasle was lound

$\square$ Yes, the prohibiled waste(s) identilied below were found.

$\square$ Putrescible waste (prohibited in U10c and Area 6 Landlills).

$\square$ Hazardous waste per NAC 444.580

$\square$ PCB waste regulaled by TSCA

$\square$ Waste containing free liquids

$\square$ TSCA-regulated

$\square$ Waste lailing the "no added radioaclivily" per the POC requirement.

$\square$ Friable asbestos (prohibited in U1Oc and Area 6 Landlills)

$\square$ Hydrocarbon soil al $>100 \mathrm{ppm}$ TPH (prohibited in 23, allowed in U10c provided less than 50 cubic yards/week are disposed)

Corrective Aclions Taken: (description, name, dale, who notified): 
DATE: $1-29-2001$

(check one) $\square$ Area 9 - U10c

DArea 6 Hydrocarbon

$\square$ Area 23 Landilil

\begin{tabular}{|c|c|c|c|c|c|c|c|}
\hline $\begin{array}{l}\text { WASTEGENERATOR } \\
\text { Name, Phone P }\end{array}$ & $\begin{array}{l}\text { WASTEOAIGIN } \\
\text { Area, Building }\end{array}$ & $\begin{array}{l}\text { WASTE } \\
\text { CODE }\end{array}$ & $\begin{array}{l}\text { TICKET } \\
\text { NUMBEA }\end{array}$ & $\begin{array}{l}\text { NET WEIGHT } \\
\text { (lbs) }\end{array}$ & TIME & $\begin{array}{l}\text { TIME } \\
\text { OUT }\end{array}$ & $\begin{array}{l}\text { Lastivep } \\
\text { Las! Name, linlthals } \\
\end{array}$ \\
\hline$B N$ & $A-22$ & 1 & 10878 & 63780 & 1450 & 6.5100 & 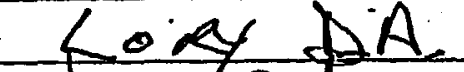 \\
\hline$B / N$ & $A-22$ & $H$ & 10879 & 65400 & 300 & 315 & 2 \\
\hline$B N$ & $0-22$ & $H$ & $10.5 \%$ & 63420 & $3: 10$ & $3: 15$ & 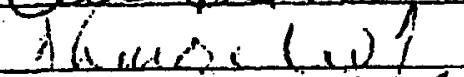 \\
\hline$\infty \not$ & $A 22$ & $\frac{1}{1-x}$ & $\mid 0881$ & $30 \%$ & $3^{3 d}$ & $3^{35}$ & Moss Jis. \\
\hline & & & & & & & \\
\hline & & & & & & & \\
\hline & & & & & & & \\
\hline & & & . & & & & \\
\hline & & & & & & & \\
\hline
\end{tabular}

"Waste Codes: ASB - Asbestos; C - Construction; H - Hydrocarbon; P - Putrescible; NP - Non-Putrescible; S - Sewage Sludge; F - FFACO

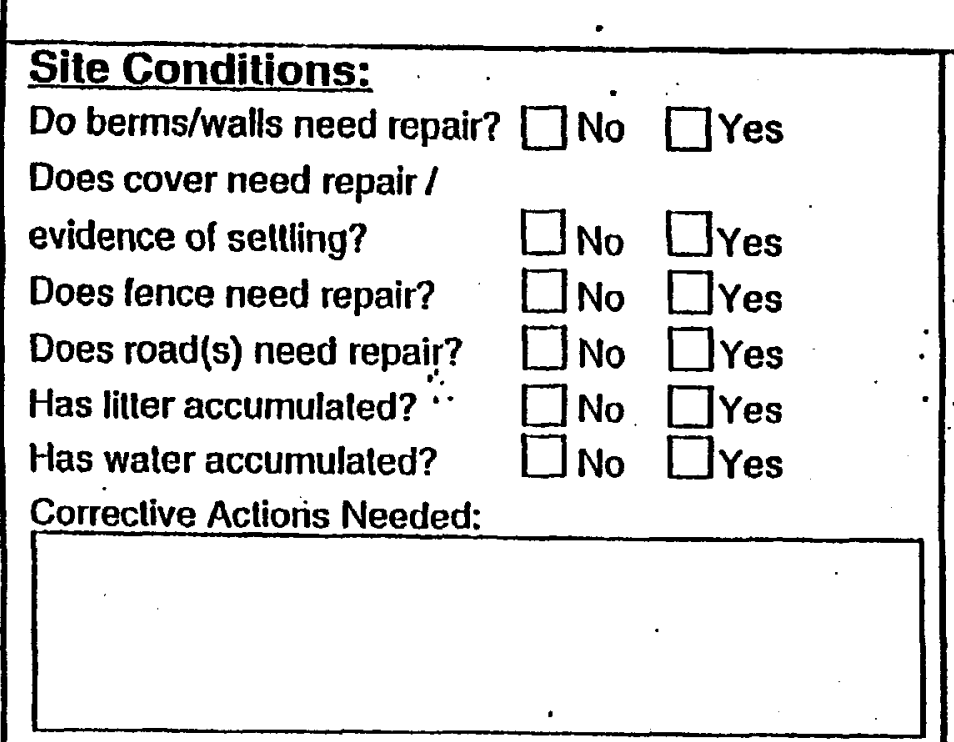

Corrective Aclions Taken: (description, name, date):

\section{INSPECTION INFORMATION}

\section{Random Load Inspection:}

$\square$ No prohibiled waste was found

$\square$ Yes, the prohibiled waste(s) idenlified below were found.

$\square$ Pulrescible wasle (prohibited in U10c and Area 6 Landfills).

$\square$ Hazardous wasle per NAC $\mathbf{4 4 4 . 5 8 0}$

Z PCB waste regulated by TSCA

$\square$ Waste containing free liquids

$\square$ TSCA-regulated

$\square$ Waste failing the "no added radioactivily" per the POC requirement.

$\square$ Friable asbeslos (prohibited in U1Oc and Area 6 Landfills)

$\square$ Hydrocarbon soil at $>100$ ppm TPH (prohibited in 23, allowed in U10c provided less than 50 cubic yards/week are disposed)

Corrective Actions Taken: (description, name, date, who notilied):
INSPECTED BY

(dale/time):
INSPECTED BY (date/time): 





LANDFILL DAILY ACCESS REGISTER

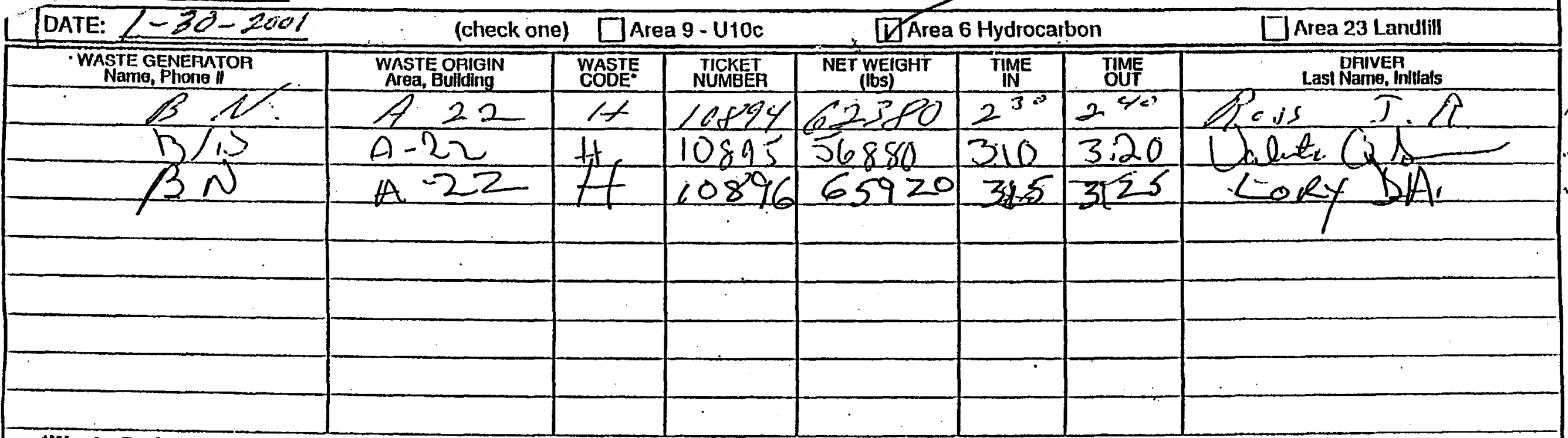

"Waste Codes: ASB -Asbestos; C - Construclion; H-Hydrocarbon; P-Pulrescible; NP - Non-Putrescible; S - Sewage Sludge; F-FFACO

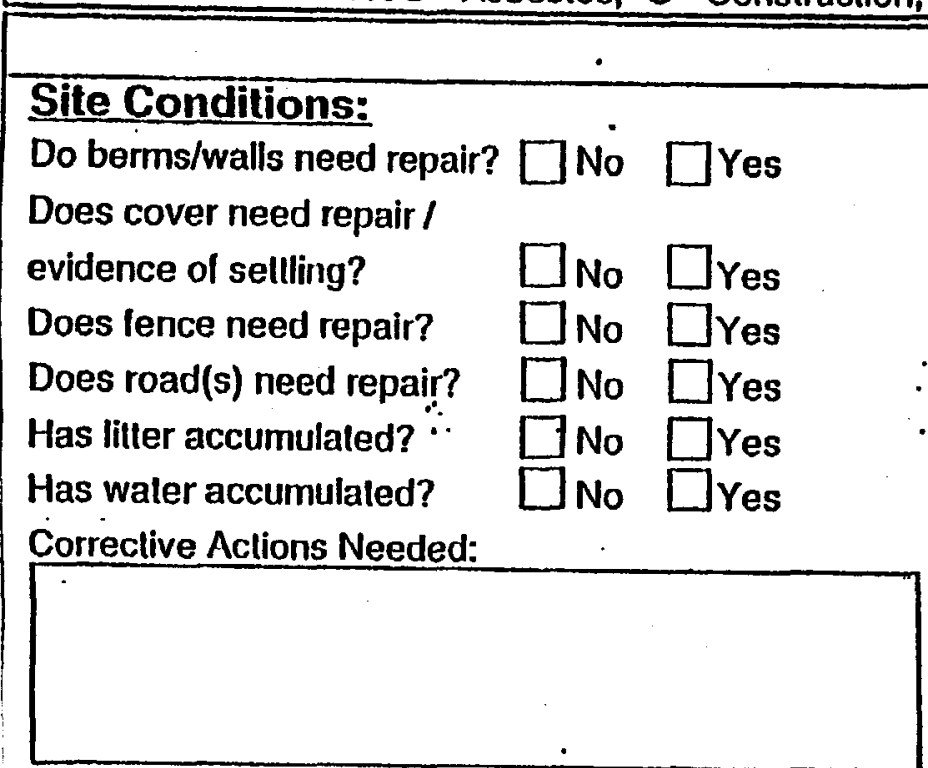

Correclive Actions Taken: (description, name, date):

\section{INSPECTED BY \\ (date/time):}

\section{INSPECTION INFOAMATION}

\section{Random Load Inspection: Ticket Number: ,}

$\square$ No prohibiled waste was found

$\square$ Yes, the prohibiled wasle(s) identified below were found.

$\square$ Pulrescible wasle (prohibiled in U10c and Area 6 Landfills).

$\square$ Hazardous wasle per NAC 444.580

$\square$ PCB wasle regulated by TSCA

$\square$ Waste containing free liquids

$\square$ TSCA-regulated

$\square$ Waste failing the "no added radioactivily" per the POC requirement.

$\square$ Friable asbestos (prohibited in U1Oc and Area 6 Landills)

$\square$ Hydrocarbon soil al >100 ppm TPH (prohibiled in 23, allowed in U10c provided less than 50 cubic yards/week are disposed)

Corrective Aclions Taken: (descriplion, name, date, who notilied): 
DATE: $|-5|-2006$ (check one) $\square$ Area 9-U10c

4 Area 6 Hydrocarbon

$\square$ Area 23 Landili

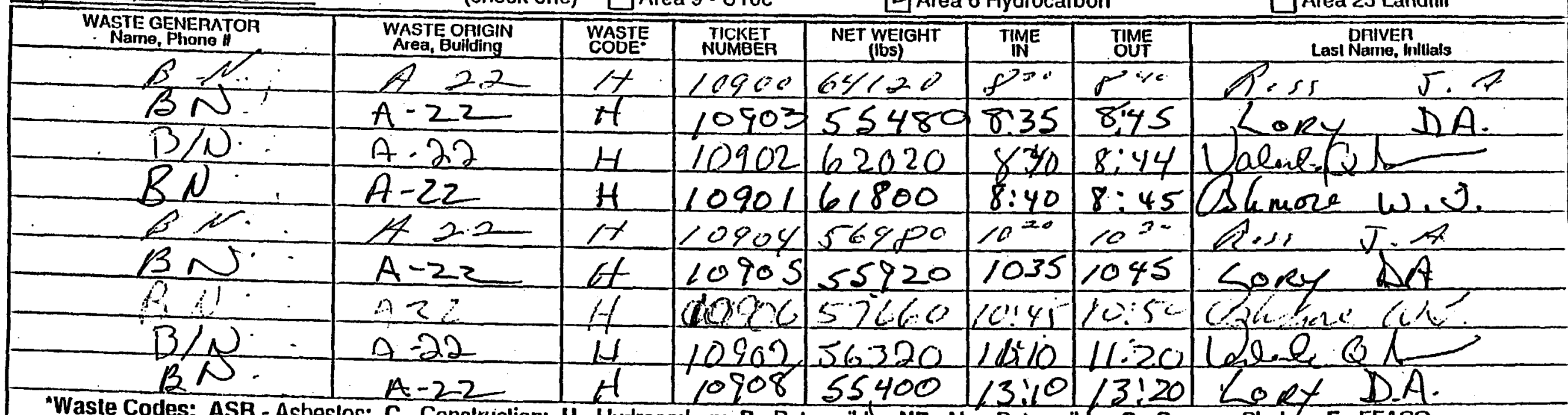

"Waste Codes: ASB -Asbeslos; C - Construction; H-Hydrocarbon; P - Pulrescible; NP - Non-Putrescible; S - Sewage Sludge; F- FFACO

\section{Sile Conditions:}

Do berms/walls need repair?

Does cover need repair/

evidence of selling?

Does fence need repair?

Does road(s) need repair?

Has litter accumulated? $\because$

Has water accumulated?

Correclive Aclions Needed:

Corrective Aclions Taken: (description, name, date):

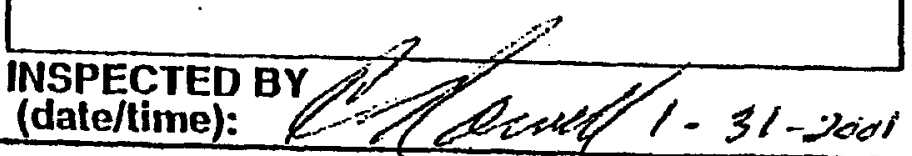

TNo $\square$ Yes

$\nabla$ No $\square$ Yes

$\square$ N6, $\square$ Yes

Dro $\square$ Yes

$\square$ No $\square$ Yes

\section{INSPECTION INFORMATION}

\section{Random Load Inspection: Ticket Number:}

\section{No prohibited waste was lound}

$\square$ Yes, the prohibiled wasle(s) identilied below were found.

$\square$ Putrescible waste (prohibiled in U10c and Area 6 Landfills).

$\square$ Hazardous waste per NAC 444.580

$\square$ PCB waste regulated by TSCA

$\square$ Wasle containing Iree liquids

$\square$ TSCA-regulated

$\square$ Waste failing the "no added radioactivity" per the POC requirement.

$\square$ Friable asbestos (prohibiled in U1Oc and Area 6 Landills)

$\square$ Hydrocarbon soil at $>100 \mathrm{ppm}$ TPH (prohibited in 23, allowed in U10c provided less than 50 cubic yards/week are disposed)

Corrective Aclions Taken: (description, name, date, who nolitied):

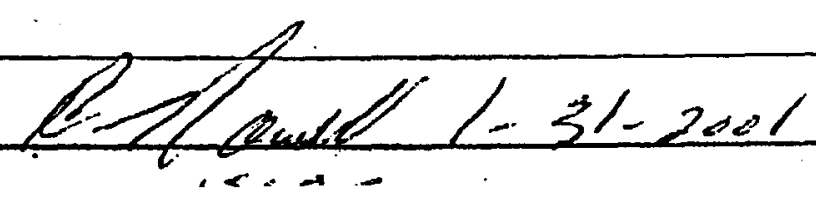




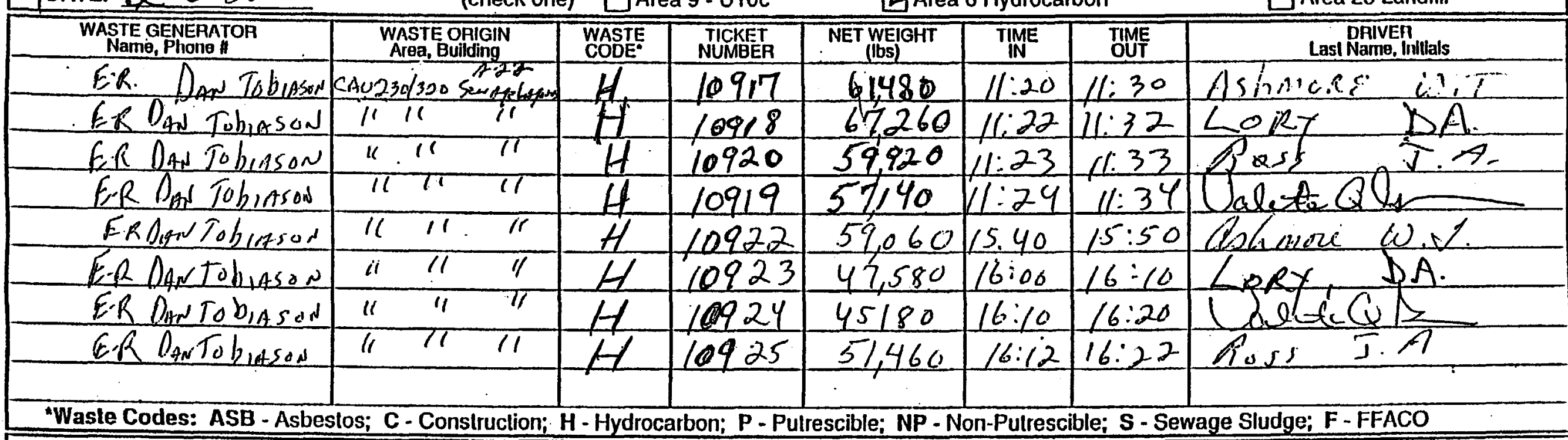

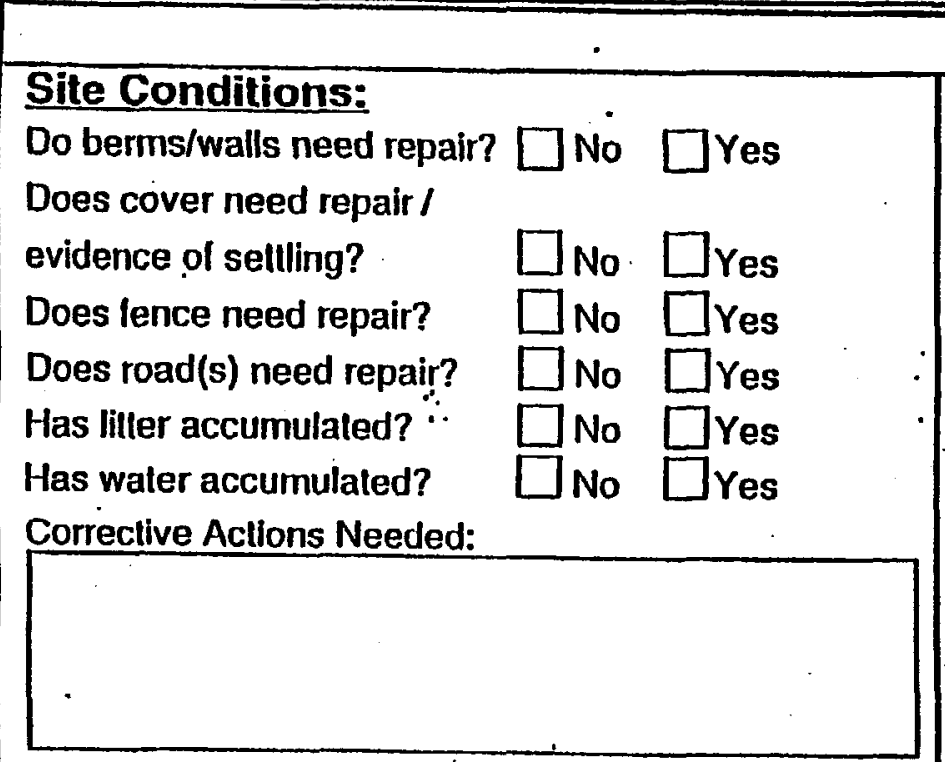

Correclive Actions Taken: (description, name, date):

\section{INSPECTION INFORMATION}

\section{Random Load Inspection: Ticket Number: '}

$\square$ No prohibited waste was lound

Yes, the prohibited waste(s) Identified below were found.

$\square$ Putrescible wasle (prohibiled in U10c and Area 6 Landfills).

$\square$ Hazardous waste per NAC 444.580

$\square$ PCB waste regulated by TSCA

$\square$ Waste containing free liquids

$\square$ TSCA-regulated

$\square$ Waste failing the "no added radioactivily" per the POC requirement.

$\square$ Friable asbestos (prohibited in U1Oc and Area 6 Landfills)

$\square$ Hydrocarbon soil at $>100 \mathrm{ppm}$ TPH (prohibited in 23, allowed in U10c provided less than 50 cubic yards/week are disposed)

Correclive Aclions Taken: (descriplion, name, date, who nolified):

\section{INSPECTED BY}

(date/time):
INSPECTED BY (date/time): 
a 8 a

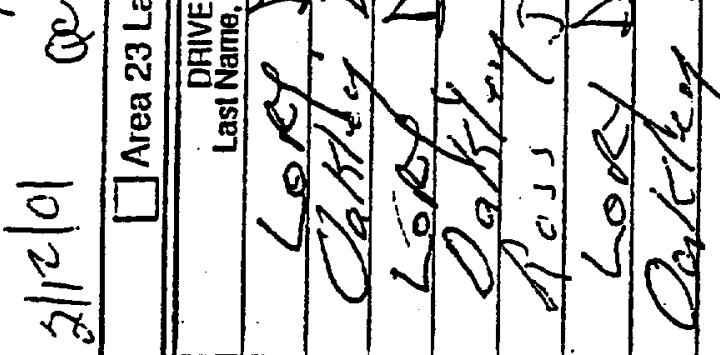

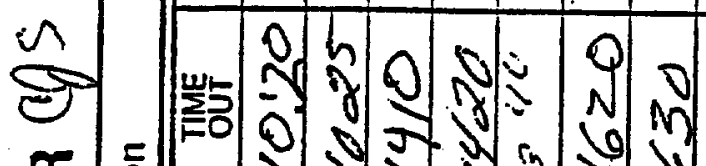

䍃

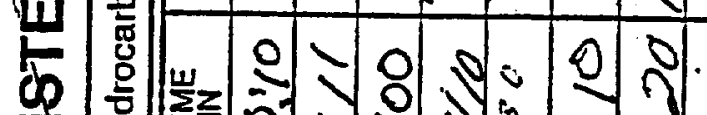

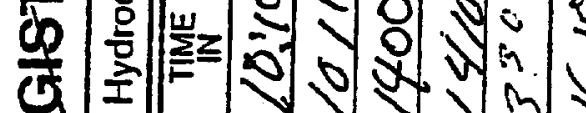

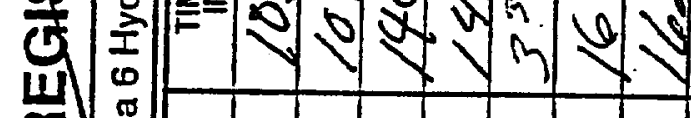

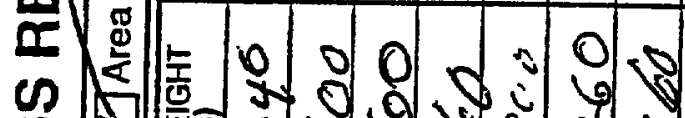

政

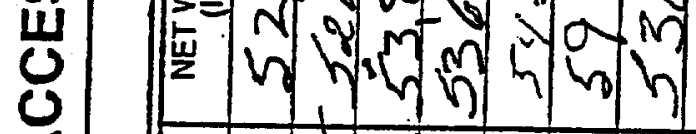

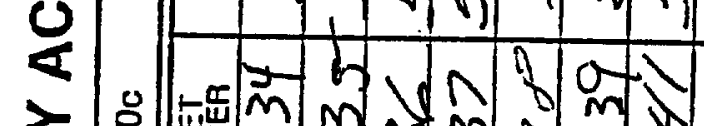

7 :

$\left.\frac{1}{a}\right)=20$

$\Delta$ (

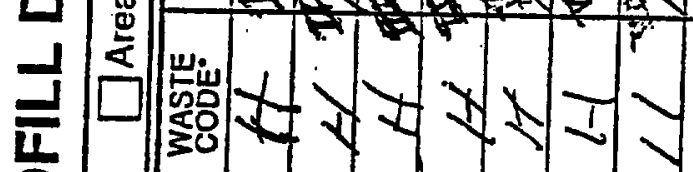

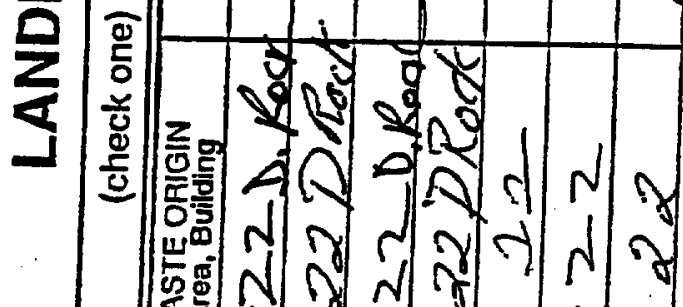

影行 $x<x<4$
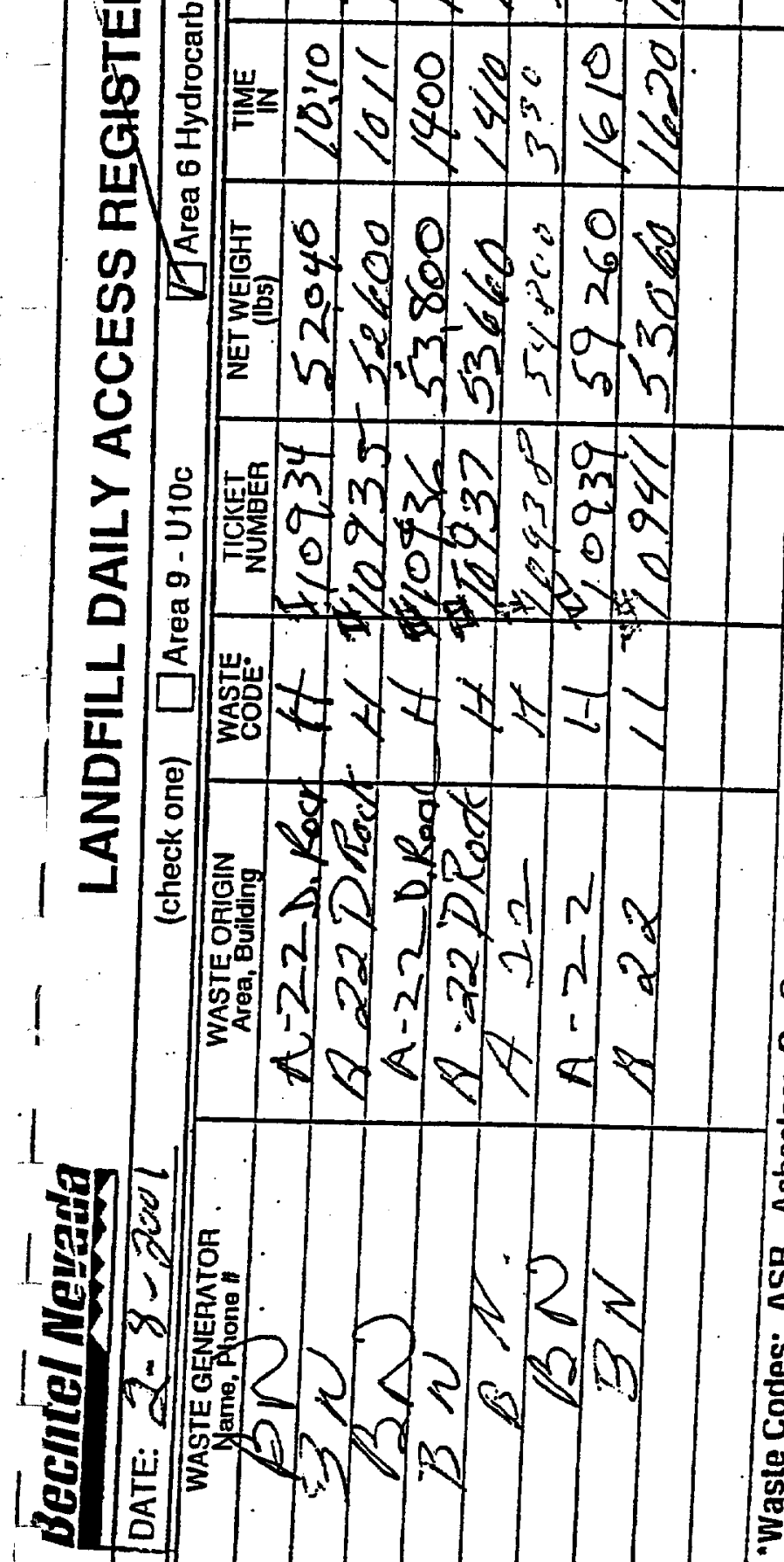

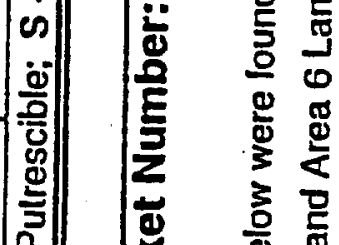

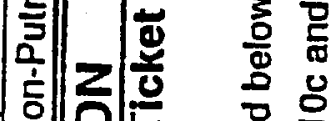

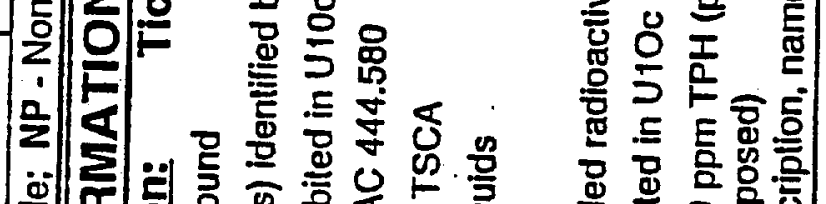

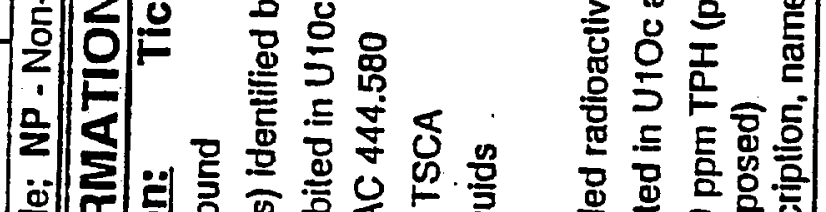

은

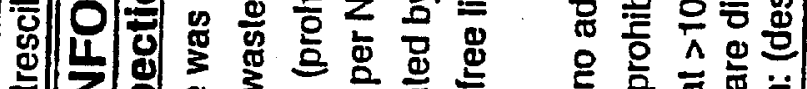

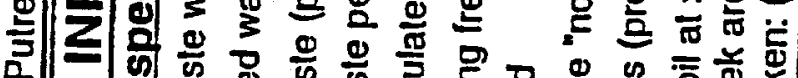

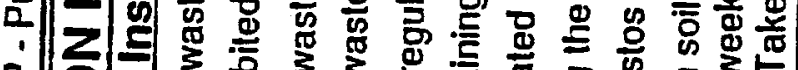

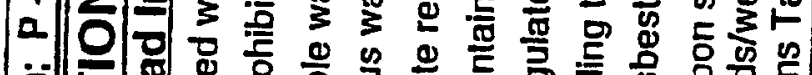

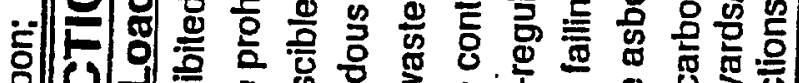

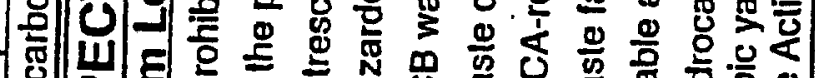

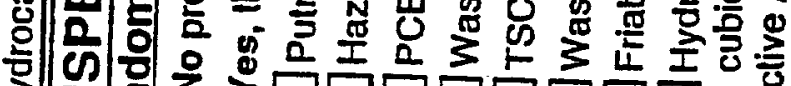

$\geq$
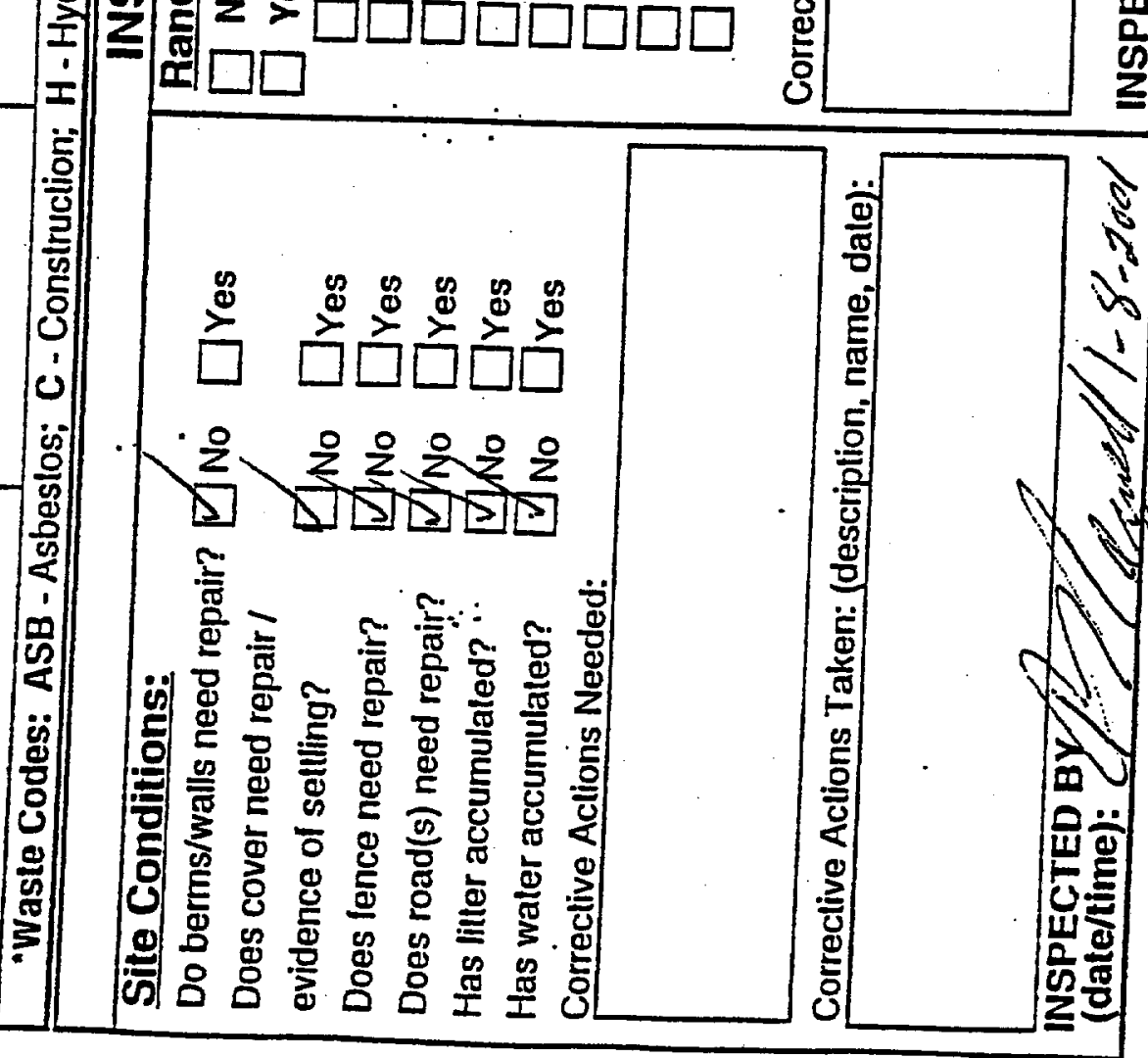


\begin{tabular}{|c|c|c|c|c|c|c|}
\hline \multirow{2}{*}{$\begin{array}{l}\text { Bechlotitevada } \\
\text { DATE: } 2-12-10\end{array}$} & \multicolumn{6}{|c|}{ LANDFILL DAILY ACCESS BEGISTER CfS $2 / 13 / 0 /$} \\
\hline & \multicolumn{6}{|c|}{ (check one) $\square$ Area 9-U10c $\quad$ [QÄrea 6 Hydrocarbon $\quad \square$ Area 23 Landlill } \\
\hline $\begin{array}{c}\text { WASTE GENERATOA } \\
\text { Name, PhonA }\end{array}$ & $\begin{array}{l}\text { WASTEOAGGIN } \\
\text { Arga, Bullding }\end{array}$ & \begin{tabular}{|l|l|} 
WASTE & TICKET \\
CODE & NUMBER \\
\end{tabular} & 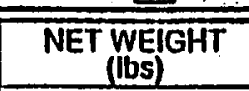 & TIME & TIME & $\begin{array}{c}\text { DAivER } \\
\text { Last Name, Inllials }\end{array}$ \\
\hline - B/N: & $A-22$ & 10943 & 50720 & $10: 00$ & $10: 15$ & $A-1822 \mathrm{GL}_{\mathrm{K}}$ \\
\hline 50 & $A-22$ & $4+10944$ & 43420 & $10120 \%$ & $10: 25$ & \multirow{2}{*}{621 Colum } \\
\hline 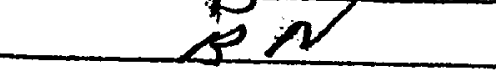 & $p+2$ & 404109425 & $5=9 * 0$ & $10 \times 25$ & 106,36 & \\
\hline$E n$ & $72 \alpha$ & 310947 & 59660 & 12,20 & 1225 & "tapar Pnge \\
\hline$B / N$ & $7-22$ & $4 \$ 11946$ & 57480 & 1230 & $12: 25$ & \multirow{2}{*}{ 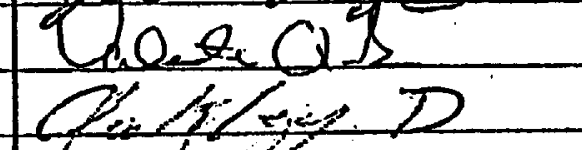 } \\
\hline$B \mu$ & $11 \cdot 12$ & t) axto942 & 63940 & 155 & $2 / 0$ & \\
\hline$B N$ & A.22 & $91004 \%$ & 54980 & $2 ! 15$ & $2: 26$ & \multirow{2}{*}{ lus 16 inn } \\
\hline & & & & & & \\
\hline "Waste Codes: ASB - A & S; C-Consiru & & & & & \\
\hline \multicolumn{7}{|c|}{ INSPECTION INFORMATION } \\
\hline \multirow{8}{*}{\multicolumn{2}{|c|}{$\begin{array}{l}\text { Site Conditions: } \\
\text { Do berms/walls need repair? } \square \text { No } \square \text { Yes } \\
\text { Does cover need repair / } \\
\text { evidence ol sellling? } \\
\text { Does fence need repair? } \square \text { No } \square \text { Yes } \\
\text { Does road(s) need repair? } \square \text { No } \square \text { Yes } \\
\text { Has litler accumulated? } \because \square \text { No } \square \text { Yes } \\
\text { Has water accumulaled? } \\
\text { Corrective Aclions Needed: }\end{array}$}} & \multirow{9}{*}{\multicolumn{5}{|c|}{$\begin{array}{l}\text { Random Load Inspection: Ticket Number: } \\
\square \text { No prohibiled waste was found } \\
\text { Yes, the prohibiled waste(s) identified below were found. } \\
\square \text { Putrescible waste (prohibiled in U10c and Area } 6 \text { Landfills). } \\
\square \text { Hazardous waste per NAC } 444.580 \\
\square \text { PCB wasle regulated by TSCA } \\
\square \text { Waste conlaining Iree liquids } \\
\square \text { TSCA-regulated } \\
\square \text { Waste failing the "no added radioaclivily" per the POC requirement. } \\
\square \text { Friable asbestos (prohibited in U1Oc and Area } 6 \text { Landfills) } \\
\square \text { Hydrocarbon soil at >100 ppm TPH (prohibited in 23, allowed in U10c provided less than } 50 \\
\text { cubic yards/week are disposed) } \\
\text { Corrective Actions Taken: (description, name, date, who notilied): }\end{array}$}} \\
\hline & & & & & & \\
\hline & & & & & & \\
\hline & & & & & & \\
\hline & & & & & & \\
\hline & & & & & & \\
\hline & & & & & & \\
\hline & & & & & & \\
\hline \multicolumn{2}{|c|}{$\begin{array}{l}\text { Correclive Aclions Needed: } \\
\end{array}$} & & & & & \\
\hline \multicolumn{2}{|c|}{ Correclive Aclions Taken: (description, name, date): } & \multicolumn{5}{|c|}{ 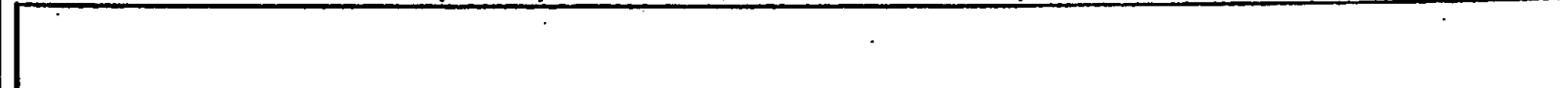 } \\
\hline \multicolumn{2}{|l|}{$\begin{array}{l}\text { INSPECTED BY } \\
\text { (date/time): }\end{array}$} & \multicolumn{5}{|c|}{ INSPECTED BY (date/time): } \\
\hline
\end{tabular}

\section{Site Conditions:}

Do berms/walls need repair? $\square$ No $\square$ Yes

Does cover need repair/

evidence of sellling?

Does fence need repair?

Does road(s) need repair?

Has filler accumulated?

Has water accumulated?

date/time:

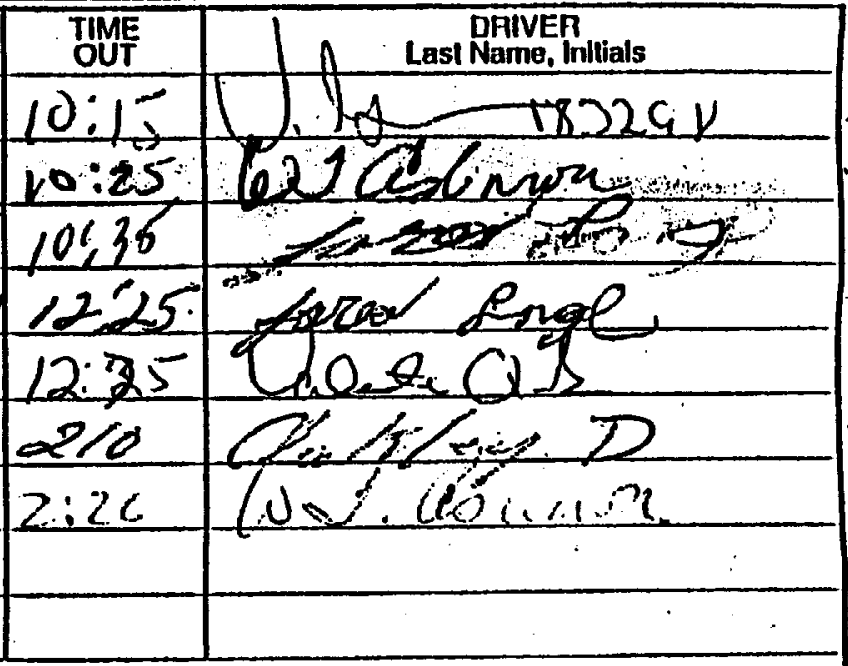

\section{INSPECTION INFORMATION}

7 No prohibiled waste was found

Yes, the prohibiled waste(s) identified below were found.

$\square$ Putrescible waste (prohibiled in U10c and Area 6 Landfills)

$\square$ Hazardous waste per NAC 444.580

$\square$ PCB waste regulated by TSCA

$\square$ Waste containing Iree liquids

TSCA-regulaled

Waste failing the "no added

C Fiable asbestos (prohibited in U1OC a

Hydrocarbon soil al $>100 \mathrm{ppm}$ TPH (prohibited in 23, allowed in U10c provt

cibic yards/week are disposed) 


\section{APPENDIX D}

\section{VERIFICATION SAMPLE ANALYTICAL REPORTS}

(Report includes analytical data only. Complete raw data is available in project file) 


\section{CIIENT: , Bechtel Nevada}

P.O. Box 98521 , M/S NTS273

ATIN: $\quad$ Ted Reding

PROJECT NAME: V1059

NEL ORDER ID: L0104091

PROJECT NUMBER: 23081

Attached are the analytical results for samples in support of the above referenced project.

Samples submitted for this project were not sampled by NEL Laboratories. Samples were received by NEL in good condition, under chain of eustody on 4/9/0l.

Should you have any questions or comments, please feel free to contact our Client Services department at (702) $657-1010$.

Some surrogate results have been thagged as follows:

D - Sample required dilution. Sample QC results were diluted outside the calibrated renge.
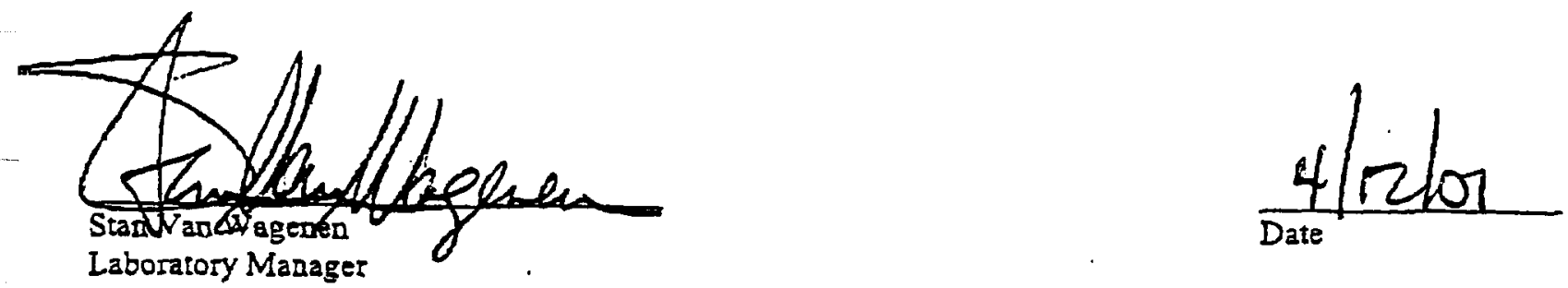

CERTIFICATIONS:

\begin{tabular}{|c|c|c|c|c|c|c|c|}
\hline & Reno & Las Vegas & S. Califormia & & Reno & Las Vegas & S. California \\
\hline Arizona & $A Z 0520$ & $A 20518$ & AZO60S & Idaho & Certified & Certified & \\
\hline Califomia & 1707 & 2002 & 2264 & Montana & Certified & Certified & \\
\hline $\begin{array}{l}\text { of Engineers } \\
\text { of }\end{array}$ & Cextified & Certified & & $\begin{array}{l}\text { Nevada } \\
\text { L.A.C.S.D. }\end{array}$ & NV033 & NV052 & $\begin{array}{l}\text { CAO84 } \\
10228\end{array}$ \\
\hline
\end{tabular}




$\begin{array}{lll}\text { IIIENT: } & \text { Bechtel Nevada } & \text { CLIENT ID: } \\ \text { PROJECT ID: } & \text { V1059 } & \text { DATE SAMPLED: 4/9/01 } \\ \text { ?ROJECT \#: } & 23081 & \text { NEL SAMPLE ID: } 10104091-01\end{array}$

IEST: $\quad$ Total Extractable Petroleum Hydrocarbons Fuel Finger Print by EPA Method $8015 \mathrm{~N}$, July 1992

VETHOD: EPA 8015M ANALYST: CCS - Las Vegas Division

VATRIX: Solid

EXTRACTED: $\quad 4 / 11 / 01$

IILUTION: 1 ANALYZED: $4 / 11 / 01$

\begin{tabular}{|c|c|c|}
\hline PARAMEIER & Result & $\begin{array}{l}\text { Reporting } \\
\text { Limit }\end{array}$ \\
\hline 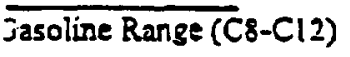 & ND & 10. $\mathrm{mg} / \mathrm{kg}$ \\
\hline Jiesel Range (C12-C22) & ND & 10. $\mathrm{mg} / \mathrm{kg}$ \\
\hline Jil Range (C12-C34) & ND & 50. $\mathrm{mg} / \mathrm{kg}$ \\
\hline iotal & ND & 10. $\mathrm{mg} / \mathrm{kg}$ \\
\hline
\end{tabular}

iurrogate

\% Recovery

111

Acceptable Range

Jetacosane

$54-130$

VD - Not Detected

his report shall not be reproduced except in full, without the wrillen approval of the laboratory. 


$\begin{array}{lll}\text { CLIENT: } & \text { Bechtel Nevads } & \text { CLIENT D: } \\ \text { PROJECT DD: } & \text { V1059 } & \text { DATE SAMPLED: } 4 / 9101 \\ \text { PROJECT \#: } & 23081 & \text { NEL SAMPIE DD: L0104091-02 }\end{array}$

TEST: Total Extractable Petroleum Hydrocarbons Fuel Finger Print by EPA Method 8015M, July 1992

METHOD: EPA 8015M ANAIYSI: CCS-Las Vegas Division

MATRD: Solid EXTRACTED: $4 / 11 / 01$

DILUTION: $1 \quad$ ANALYZED: $4 / 11 / 01$

\begin{tabular}{|c|c|c|}
\hline PARAMETER & Result & $\begin{array}{c}\text { Reportlng } \\
\text { Limit }\end{array}$ \\
\hline Gasoline Range (CB-C12) & ND & 10. $\mathrm{mg} / \mathrm{kg}$ \\
\hline Dicsel Range (C12-C22) & ND & 10. $\mathrm{mg} / \mathrm{kg}$ \\
\hline Oil Range (C12-C34) & ND & 50. $\mathrm{mg} / \mathrm{kg}$ \\
\hline Total & ND & 10. $\mathrm{mg} / \mathrm{kg}$ \\
\hline \multicolumn{3}{|c|}{ QUALITY CONTROL DATA: } \\
\hline Surrogate & $\%$ Recovery & Acceptable Range \\
\hline Jetacosane & 105 & $54-130$ \\
\hline
\end{tabular}

VD - Not Detected

This repor shall not be reproduced except in full, without the written approval of the laboratory. 
ILIENT: Bechtel Nevada

PROJECT ID: . V1059

?ROJECT H: 23081

IEST:

METHOD:

NAIRIX:

DILUTION:

EPA 8015M

Solid

1
Total Extractable Petroleum Hydrocarbons Fuel Finger Print by EPA Method 80151, July 1992

PARAMETER

Jasoline Range (C8-C12)

Jiesel Range (C12-C22)

Jil.Range (Cl2-C34)

Total

ZUALITY CONTROL DATA:

jurrogate

Jctacosane

$\%$ Recovery
112

ACIED:

CCS - Las Vegas Division

ANALYZED:

$4 / 11 / 01$

3205B-18

DATE SAMRLED: $4 / 9 / 01$

\begin{tabular}{l} 
Result \\
\hline ND \\
ND \\
ND \\
ND
\end{tabular}

Reporting

Limit

10. $\mathrm{mg} / \mathrm{kg}$

10. $\mathrm{mg} / \mathrm{kg}$

50. $\mathrm{mg} / \mathrm{kg}$

10. $\mathrm{mg} / \mathrm{kg}$

\section{Acceptable Range}

$54 \cdot 130$

ND - Nor Detected

This report shall not be reproduced except in full, without the writien approval of the laboratory. 


\begin{tabular}{|c|c|c|}
\hline $\begin{array}{l}\text { CLIENT: } \\
\text { PROIECT D: } \\
\text { PROJECT \#: }\end{array}$ & $\begin{array}{l}\text { Bechtel Nievada } \\
\text { V1059 } \\
23081\end{array}$ & $\begin{array}{ll}\text { CLIENT ID: } & 320 S B-19 \\
\text { DAIE SAMPLED: } & 4 / 9 / 01 \\
\text { NEI SAMPLE ID: } & \text { L0104091-04 }\end{array}$ \\
\hline
\end{tabular}

TEST: Total Extractable Petroleum Hydrocarbons Fuel Finger Print by EPA Method 8015 MI, July 1992 VETHOD: EPA 8015M ANALYST: CCS - Las Vegas Division VATREX: Solid EXTRACTED: 4/11/01

JILUTION: 1 . ANALYZED: $4 / 11 / 01$

PARAMEIER Rerting

\section{?ARAMETER}

jasoline Range (C8-C12)

Jiesei Range (C12-C22)

Jil Range (C12-C34)

ional

2UALITY CONTROL DATA:

jurrogate

Jetacosane

\begin{tabular}{l} 
Resuit \\
\hline$N D$ \\
$N D$ \\
$N D$ \\
$N D$
\end{tabular}

$\%$ Recovery

108
Reporting

10. $\mathrm{mg} / \mathrm{kg}$

10. $\mathrm{mg} / \mathrm{kg}$

50. $\mathrm{mg} / \mathrm{kg}$

10. $\mathrm{mg} / \mathrm{kg}$

\section{Acceptable Range}

$54-130$

ND - Not Detected

his report shall not be reproduced except in full, without the written approval of the laboratory. 
IIENT: Bechtel Nevada

'ROJECI ID: V1059

'ROJECT \#: 2308 I
CLIENT D: $\quad 320 S B-20$

DATE SAMPLED: 4/9/01

NEL SA.MPIE ID: L0104091-05
IEST:

METHOD:

IAIRIX:

ILUTION:
Total Extractable Petroleum Fydrocarbons Fuel EPA $8015 \mathrm{M}$ Solid 1
ANAIYST:

EXTRACTED:

ANAIYZED:

ANALYZED: $\quad 4 / 11 / 01$

?ARAMETER

j2soline Range (C8-C12)

Jiesel Range (C12-C22)

Jil Range (C12-C34)

"otal

DUALTY CONTROL DATA:

iurrogate

Jetacosane

\begin{tabular}{l} 
Result \\
\hline ND \\
ND \\
ND \\
ND
\end{tabular}

$\%$ Recovery

123
ND - Nor Detected

his report shall not be reproduced except in full, without the wrtten approval of the laboratory.
CCS - Las Vegas Division

$4 / 11101$ 1101

Reporting

Limit

10. $\mathrm{mg} / \mathrm{kg}$

10. $\mathrm{mg} / \mathrm{kg}$

50. $\mathrm{mg} / \mathrm{kg}$

10. $\mathrm{mg} / \mathrm{kg}$

\section{Acceptable Range}

$54-130$ 


\begin{tabular}{|c|c|c|}
\hline $\begin{array}{l}\text { CLIENT: } \\
\text { PROJECI D: } \\
\text { PROJECI } \pm:\end{array}$ & $\begin{array}{l}\text { Beehtel Nevada } \\
\text { V1059 } \\
23081\end{array}$ & $\begin{array}{ll}\text { CLIENT ID: } & \text { 320SB-21 } \\
\text { DATE SAMPIED: } & \text { 4/9/01 } \\
\text { NEL SA.MPIE ID: } & \text { L0104091-06 }\end{array}$ \\
\hline
\end{tabular}

TEST: Total Extractable Petroleum Hydrocarbons Fuel Finger Print by EPA Method 8015M. July 1992

METHOD: EPA $8015 \mathrm{M}$ ANAIYST: CCS - Las Vegas Divisior

MATREX: Solid EXTRACTED: 4/11/01

DLUTION: 1 ANALYZED: A/11/01

PARAMTER Reporting

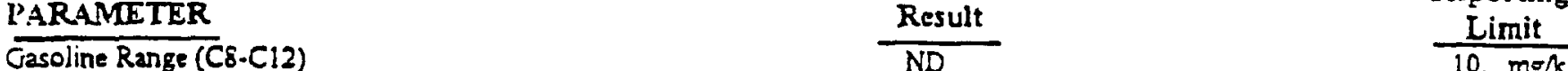

Gasoline Range (C8.C12) ND $\quad \frac{\mathrm{R}}{\mathrm{ND}} \quad \frac{\mathrm{mg} / \mathrm{kg}}{10 . \mathrm{mgg}}$

Diesel Range (C12-C22) ND $\quad$ ND

Oil Range (C12-C34) $\quad$. ND $\quad 50 . \mathrm{mg} / \mathrm{kg}$

Sotal ND

10. $\mathrm{mg} / \mathrm{kg}$

QUALITY CONTROL DATA:

Surrogate

\% Recovery

Octacosane

107

Acceptable Range

$54-130$

ND - Not Detected

This report shall not be reproduced except in full, without the written approval of the laboratory. 


\begin{tabular}{|c|c|c|}
\hline $\begin{array}{l}\text { :IIENT: } \\
\text { ROJECT ID: } \\
\text { ROJECT } \#:\end{array}$ & $\begin{array}{l}\text { Bechtel Nevada } \\
\text { V1059 } \\
23081\end{array}$ & $\begin{array}{ll}\text { CLIENT ID: } & \text { 320SB-22 } \\
\text { DATE SAMPLED: } 4 / 9101 \\
\text { NEL SAMPLE ID: L0104091-07 }\end{array}$ \\
\hline
\end{tabular}

EST: Total Extractable Petroleum Hydrocarbons Fuel Finger Print by EPA Method 8015M, July 1992

IETHOD: EPA 8015M ANALYST: CCS - Las Vegas Division

1ATRIX: - Solid EXTRACTED: 4/11/01

IILUTION: 1 ANAIYZED: 4/11/01

\begin{tabular}{lll}
\hline 'ARAMETER & Result & Reporting \\
\cline { 2 - 3 } jasoline Range (C8-C12) & ND & $\frac{\text { Limit }}{10 . \mathrm{mg} / \mathrm{kg}}$ \\
Jiesel Range (C12-C22) & ND & $10 . \mathrm{mg} / \mathrm{kg}$ \\
Jil Range (C12-C34) & ND & $50 . \mathrm{mg} / \mathrm{kg}$ \\
Ootal & ND & $10 . \mathrm{mg} / \mathrm{kg}$ \\
\hline
\end{tabular}

ZUALITY CONTROL DATA:

iurrogate

\% Recovery

107

Acceptable Range

Jetacosane

$54 \cdot 130$

VD - Not Detected

This report shall not be reproduced except in full, without the writter approval of the laboratory.

8. 


\begin{tabular}{|c|c|c|}
\hline $\begin{array}{l}\text { IIENT: } \\
\text { ROJECT ID: }\end{array}$ & $\begin{array}{l}\text { Bechtel Nevada } \\
\text { V1059 } \\
23081\end{array}$ & $\begin{array}{ll}\text { CLIENT ID: } & 320 S B-23 \\
\text { DATE SAMPLED: } & 4 / 9101 \\
\text { NEL SAMPLE ID: } & \text { L0104091-08 }\end{array}$ \\
\hline
\end{tabular}

EST 1ETHOD: EPA 8015M IATRR: Solid

Total Extractable Petroleum Hydrocarbons Fuel Finger Print by EPA Method 8015M, July 1992 JILUTION: 1 ANALYST: $\quad$ CCS - Ias Vegas Division EXTRACTED: $\quad 4 / 11 / 01$ ANALYZED: $\quad 4 / 11 / 01$

\begin{tabular}{|c|c|c|}
\hline 'ARAMETER & Result & $\begin{array}{l}\text { Reporting } \\
\text { Limit }\end{array}$ \\
\hline$\overline{\text { jasoline Range (C } \delta-C 12)}$ & ND & 10. $\mathrm{mg} / \mathrm{kg}$ \\
\hline Jiesel Range ( $\mathrm{Cl2}-\mathrm{C} 22)$ & ND & 10. $\mathrm{mg} / \mathrm{kg}$ \\
\hline Jil Range (Cl2-C34) & ND & 50. $\mathrm{mg} / \mathrm{kg}$ \\
\hline Cotal & ND & 10. $\mathrm{mg} / \mathrm{kg}$ \\
\hline
\end{tabular}

2UALTY CONTROL DATA:

iurrogate

$\%$ Recovery

107

Acceptable Range

Jctacosane

107

$54 \cdot 130$

\section{ND - Not Detected}

This report shall not be reproduced except in full. without the written approval of the laboratory. 


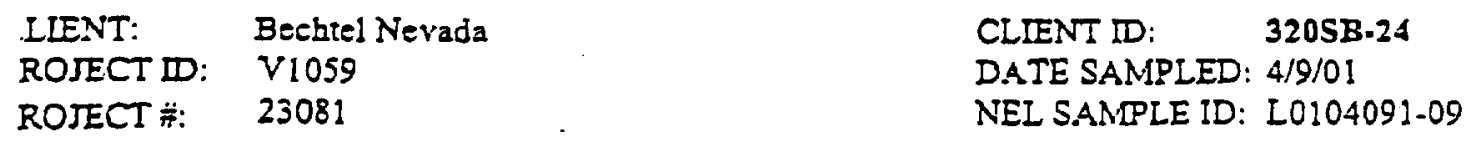

'EST: $\quad$ Total Extractnble Petroleum Hydrocarbans Fuel Finger Print by EPA Method 8015M, July 1992

IETHOD: . EPA $3015 \mathrm{M}$ ANAIYST: CCS - Las Vegas Division

LATRIX: Solid

EXTRACTED: $\quad 4 / 11 / 01$

ILUTION: 1 ANALYZED: $4 / 11 / 01$

\begin{tabular}{|c|c|c|}
\hline 'ARANIETER & Result & $\begin{array}{l}\text { Reporting } \\
\text { Limit }\end{array}$ \\
\hline iasoline Range (C8-C12) & ND & 10. $\mathrm{mg} / \mathrm{kg}$ \\
\hline riesel Range (C12-C22) & ND & 10. $\mathrm{mg} / \mathrm{kg}$ \\
\hline jil Range (C]2-C34) & ND & 50. $\mathrm{mg} / \mathrm{kg}$ \\
\hline otal & ND & 10. $\mathrm{mg} / \mathrm{kg}$ \\
\hline
\end{tabular}

?UALITY CONTROL DATA:

iurrogate

$\%$ Recovery

Acceptoble Range

Jetacosane

117

$54 \cdot 130$

JD - Not Deteeted

This report shall not be reproduced except in full, without the written approval of the laboratory. 


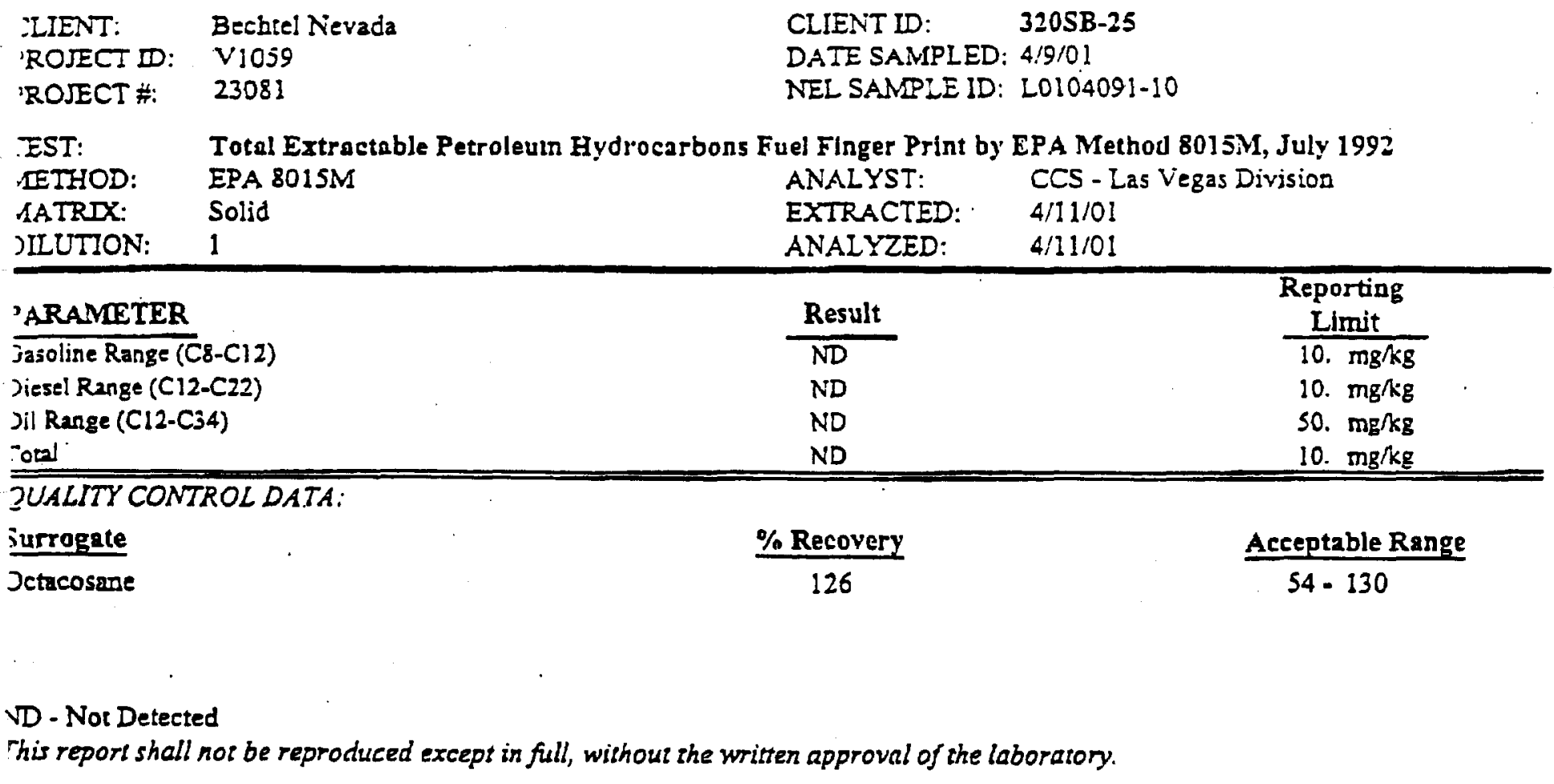




$\begin{array}{lll}\text { LIENT: } & \text { Bechrel Nevada } & \text { CLIENT ID: } \\ \text { ROJECT ID: } & \text { Vi059 } & \text { DATE SAMPLED: 4/9/01 } \\ \text { ROJECT \#̈: } & 23081 & \text { NEL SAMPLE ID: L0104091-11 }\end{array}$

EST: Total Extractable Petroleum Hydrocarbons Fuel Finger Print by EPA Method 8015M, Juy 1992

ETHOD: EPA 8015M ANALYST: CCS - Las Vegas Division

IATRX: Solid EXTRACTED: 4/11/01

ILUTION: $1 \quad$ ANALYZED: $4 / 12 / 01$

\begin{tabular}{|c|c|c|}
\hline ARAMETER & Result & $\begin{array}{c}\text { Reporting } \\
\text { Limit }\end{array}$ \\
\hline asoline Range (C8-C12) & ND & 10. $\mathrm{mg} / \mathrm{kg}$ \\
\hline iesel Range (C12.C22) & ND & 10. $\mathrm{mg} / \mathrm{kg}$ \\
\hline i) Range (C12-C34) & ND & 50. $\mathrm{mg} / \mathrm{kg}$ \\
\hline otal & ND & 10. $\mathrm{mg} / \mathrm{kg}$ \\
\hline
\end{tabular}

UALITY CONTROL DATA:

urrogate

ictacosine
$\%$ Recovery

112
Acceptable Range $54-130$

ID - Not Detected

his report shall not be reproduced except in full, without the written approval of the laboratory. 


\begin{tabular}{|c|c|c|}
\hline $\begin{array}{l}\text { LIENT: } \\
\text { ROJECT D: } \\
\text { ROJECT H: }\end{array}$ & $\begin{array}{l}\text { Bechrel Nevada } \\
\text { V1059 } \\
23081\end{array}$ & $\begin{array}{ll}\text { CLIENT ID: } & \text { 320SB-27 } \\
\text { DATE SAMPLED: } & 4 / 9 / 01 \\
\text { NEL SAMPLE ID: } & \text { I0104091-12 }\end{array}$ \\
\hline
\end{tabular}

EST: Total Extractable Petroleum Hydrocarbons Fuel Finger Print by EPA Method 8015M, July 1992 IETHOD: EPA 8015M IATRIX: Solid ANAIYSI: $\quad$ CCS - Les Vegas Division ILUTION: 1

EXTRACTED: 4/11/01

ANALYZED: $\quad 4 / 12 / 01$

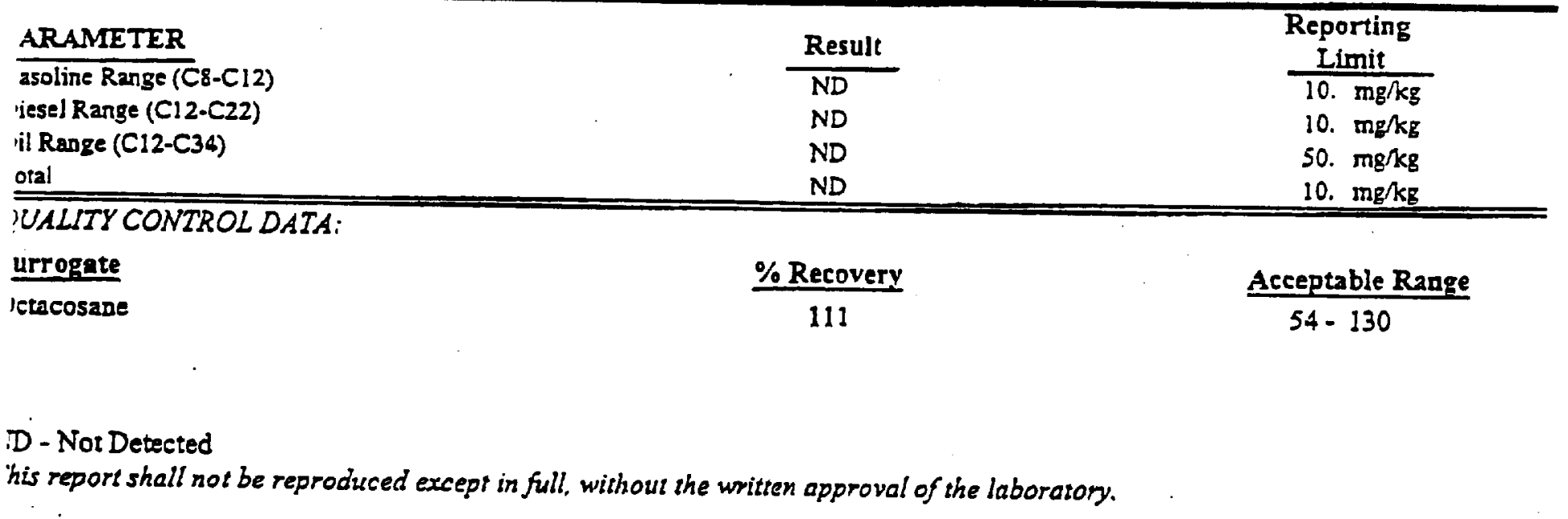




$\begin{array}{llll}\text { IIENT: } & \text { Bechtel Nevada } & \text { CLIENT ID: } & \text { 320SB-28 } \\ \text { ROJECT ID: } & \text { V1059 } & \text { DATE SAMPLED: 4/9/01 } \\ \text { ROJECT \#: } & 23081 & \text { NEL SAMPLE ID: L0104091-13 }\end{array}$

EST: Total Extractable Petroleum Hydrocarbons Fuel Finger Print by EPA Method 8015M, July 1992

IETHOD: EPA 8015M ANALYST: CCS - Las Vegas Division

IATRX: Solid EXTRACTED: 4/11/01

IILUTION: 1 ANAIYZED: $4 / 12 / 01$

\begin{tabular}{lll}
\hline 'ARAMETER & Result & Reporting \\
\cline { 2 - 3 } jasoline Range (C8-C12) & ND & $\frac{\text { Limit }}{10 . \mathrm{mg} / \mathrm{kg}}$ \\
Jiesel Range (C12-C22) & ND & $10 . \mathrm{mg} / \mathrm{kg}$ \\
Jil Range (C12-C34) & ND & $50 . \mathrm{mg} / \mathrm{kg}$ \\
otal & ND & $10 . \mathrm{mg} / \mathrm{kg}$ \\
\hline
\end{tabular}

2UALITY CONTROL DATA:

jurrogate

\% Recovery

Jetrososane

120

Acceptable Range

$54-130$

ND - Not Detected

This report shall not be reproduced except in full, without she written approval of the laboratory. 


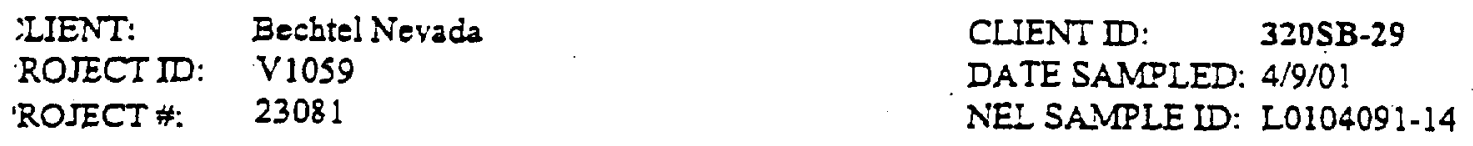

EST: Totsl Extractable Petroleum Hydrocarbons Fuel Finger Print by EPA Method 8015M, July 1992

IETHOD: EPA 8015M

IATRIX: Solid

JILUTION: $\quad 9.8$

ANAIYST: CCS - Las Vegas Dirision

EXTRACTED: $\quad 4 / 11 / 01$

ANAIYZED: $\quad 4 / 12 / 01$

\section{'ARAMETER}

jasoline Range (C $8-C 12)$

Jiesel Range (C12-C22)

Jil Range (C12-C34)

otal

UALTY CONTROL DATA:

iursogate

Jetacosane

D - Not Decected

his report shall not be reproduced except in full, without the written approval of the laboratory.

\begin{tabular}{|c|c|c|}
\hline \multicolumn{2}{|c|}{ Result } & $\begin{array}{c}\text { Reporting } \\
\text { Limit }\end{array}$ \\
\hline ND & & 98. $\mathrm{mg} / \mathrm{kg}$ \\
\hline$N D$ & & 98. $\mathrm{mg} / \mathrm{kg}$ \\
\hline 2100 & $\mathrm{mg} / \mathrm{kg}$ & 490. $\pi \mathrm{ge} / \mathrm{kg}$ \\
\hline 2100 & $\mathrm{mg} / \mathrm{kg}$ & 98. $\mathrm{mg} / \mathrm{kg}$ \\
\hline
\end{tabular}

\% Recovery

D

Acceptable Range

$54 \cdot 130$ 


\begin{tabular}{|c|c|c|}
\hline $\begin{array}{l}\text { IIENT: } \\
\text { ROJECT D: } \\
\text { ROJECT \#: }\end{array}$ & $\begin{array}{l}\text { Bechtel Nevada } \\
\text { V1059 } \\
23081\end{array}$ & $\begin{array}{ll}\text { CLIENT ID: } & \text { 320SB-30 } \\
\text { DATE SAMIPLED: } & \text { 4/9/01 } \\
\text { NEL SAMPLE ID: } & \text { L010409!-15 }\end{array}$ \\
\hline
\end{tabular}

EST: Total Extractable Petroleum Hydrocarbons Fuel Finger Print by EPA Method $8015 \mathrm{SM}$, July 1992

IETHOD: EPA 8015M ANAIYST: CCS - Las Vegas Division

LATRIX: Solid EXTRACTED: 4/11/01

IILUTION: $8.8 \quad$ ANALYZED: $4 / 11 / 01$

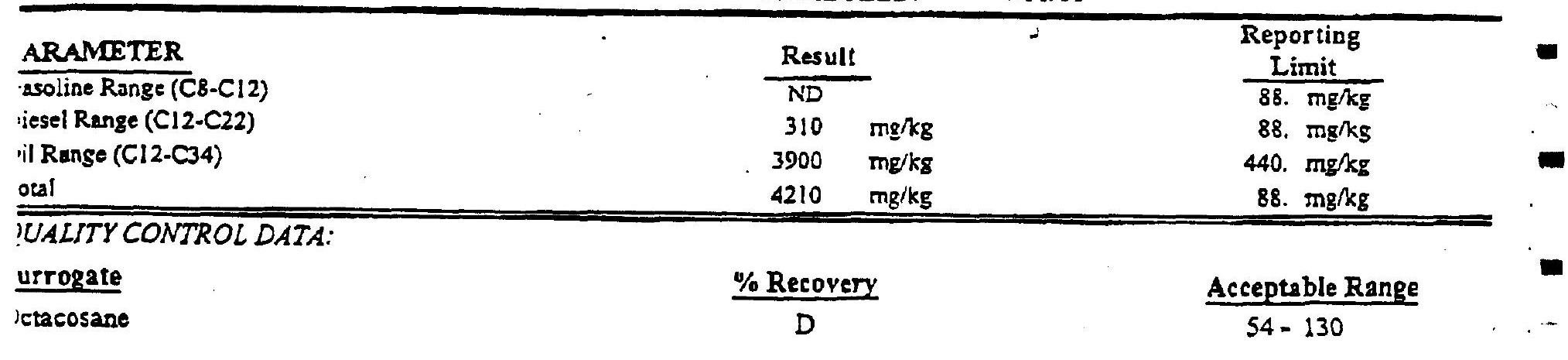

D. Not Detected

his report shall not be reproduced except in full, without the written approval of the laboratory. 


\begin{tabular}{|c|c|c|c|}
\hline 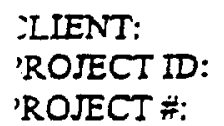 & $\begin{array}{l}\text { Beehtel Nevada } \\
\text { V1059 } \\
23081\end{array}$ & $\begin{array}{l}\text { CLIENT ID: } \\
\text { DAIE SAMPIED: } \\
\text { NEL SAMPLE ID: }\end{array}$ & $\begin{array}{l}\text { Method Blank } \\
\text { N.A } \\
010411 \text { TPHS-FP-BLK }\end{array}$ \\
\hline
\end{tabular}

:EST:

AETHOD:

-1ATRR:
Total Extractable Petroleum Hydrocarbons Fue EPA 8015M

Solid
Finger Print by EPA Method 8015M, July 1992 ANAIYST: $\quad$ CCS - Las Vegas Division EXTRACTED: $\quad 4 / 11 / 01$ ANAIYZED: $\quad 4 / 11 / 01$

\begin{tabular}{|c|c|c|}
\hline 'ARAMETER & Result & $\begin{array}{l}\text { Reporting } \\
\text { Limit }\end{array}$ \\
\hline issoline Range (C8-Cl2) & ND & 10. $\mathrm{mg} / \mathrm{kg}$ \\
\hline Jiesel Range (C12-C22) & ND & 10. $\mathrm{mg} / \mathrm{kg}$ \\
\hline Jil Range (C12.C34) & ND & 50. $\mathrm{mg} / \mathrm{kg}$ \\
\hline otal & ND & 10. $\mathrm{mg} / \mathrm{kg}$ \\
\hline
\end{tabular}

ZUALITY CONTROL DATA:

iurrogate

Jetacosane
$\%$ Recovery

94
Acceptable Range

$54-130$

JD - Not Detected

"his report shall not be reproduced except in full, without the written approval of the laboratory. 
-IENT: Bechtel Nevada

IOJECT D: V1059

2OJECI \#: 23081

EST: - Total Extractable Petroleum Hydrocarbons Fuel Finger Print by EPA Method 8015M, July 1992 ATRDX: Solid

\begin{tabular}{|c|c|c|c|c|c|c|}
\hline ARAMETER & NEL Sample ID & $\begin{array}{c}\text { Splke } \\
\text { Amount }\end{array}$ & $\frac{\text { Spike }}{\text { Result }}$ & $\frac{\text { Percent }}{\text { Recovery }}$ & $\frac{\text { Acceptable }}{\text { Range }}$ & $\underline{\text { RPD }}$ \\
\hline iesel Range (C12-C22) & 010411TPHS-FP-LCS & 166.7 & 120 & 72 & $53-91$ & \\
\hline iesel Range (C12-C22) & 010411TPHS-FP-LCSD & 166.7 & 107 & 64 & $53-91$ & 11.5 \\
\hline iesel Range (C12-C22) & L0104091-10.MS & 166.7 & 130 & 78 & $34 \cdot 114$ & \\
\hline iesel Range (C12-C22) & L0104091-10-MSD & 166.7 & 135 & 81 & $34-114$ & 3.8 \\
\hline Jtal & 010411TPHS-FP-LCS & 166.7 & 120 & 72 & $53-91$ & \\
\hline لנמכ & 010411 TPHS-FP-LCSD & 166.7 & 107 & 64 & $53-91$ & 11.5 \\
\hline stal & L0104091-10-MS & 166.7 & 130 & 78 & $34-114$ & \\
\hline stal & L0104091-10-MSD & 166.7 & 135 & 81 & $34 \cdot 114$ & 3.8 \\
\hline
\end{tabular}




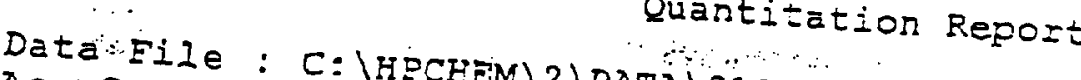

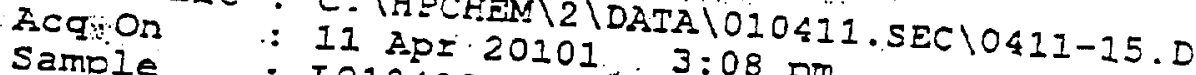

MISC : SOIL SAMPLE

IntFile: events.e

Quant Time: events.e

Quant Method : C:IHPCHEM 2UMETHODSITPOIOIR2 11 16:07 2001 Quant Results File: TPOI01R2.RES
Title

Last Update

Response via

DataAcg Meth

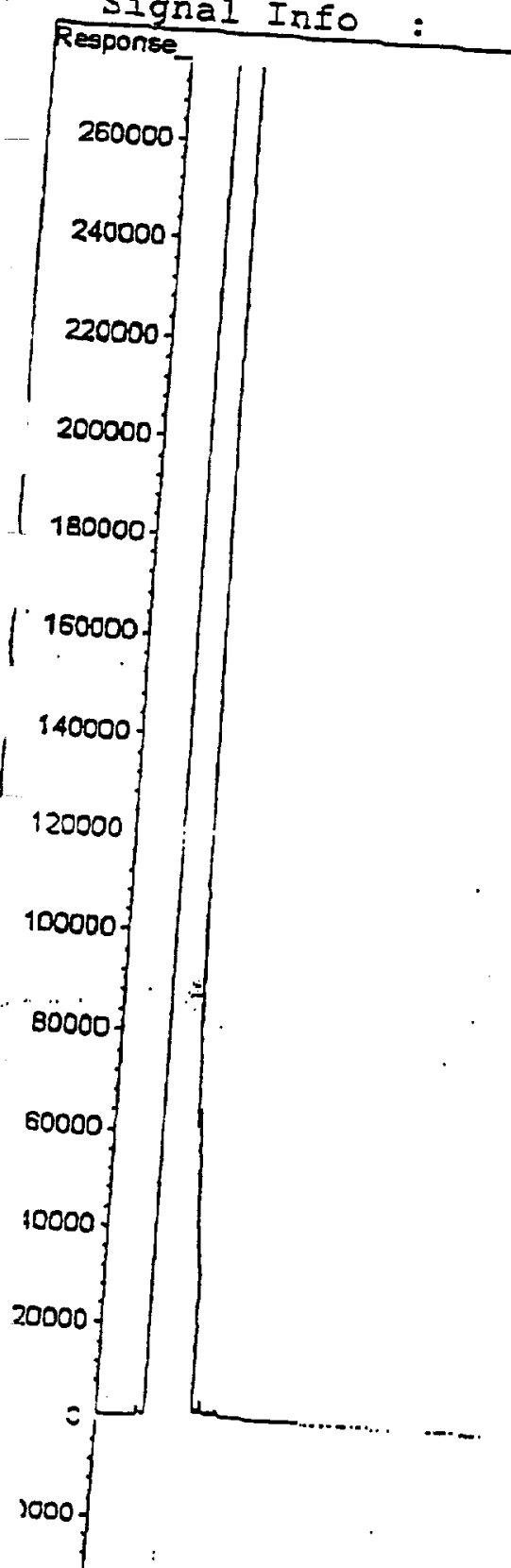

6/21/99 5eymour ErOnt TPH
Wed Jan 10 15:10.47 (Chemstation Integrator)

Multiple Io $15: 10: 472001$

TP9907R1.M
Oial $\quad 7$

rator: cos

Inst : Seymove

Muleiplr: 1.00

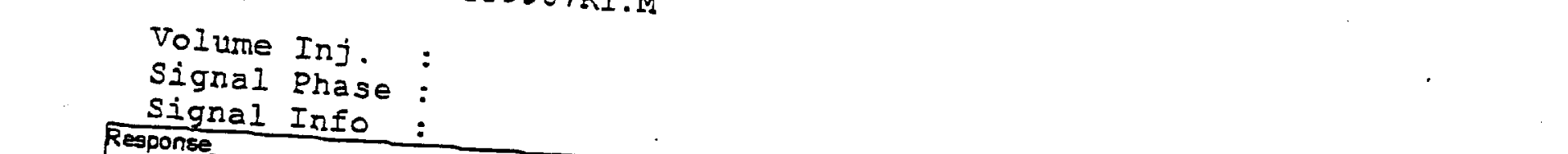

Volume Inj.

Signal Phase:

Signal Info: 


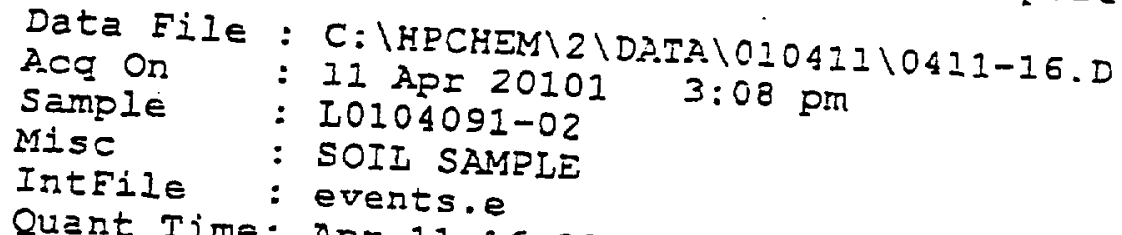

\section{Quantitation Repozt}

Quant Time: Apr 11 16:08 2001

Quant Results File: TPO101Ez.FEs

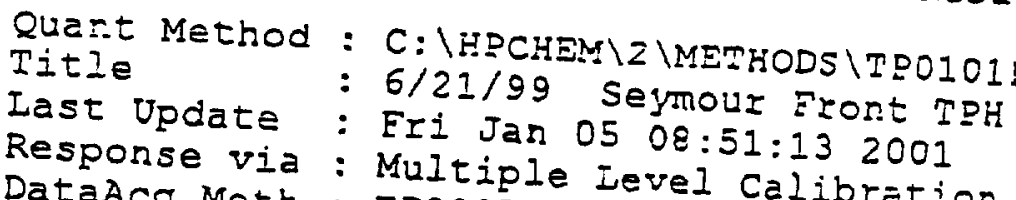

DataAcq Meth : TP9907R1.M Lel Calibration

Volume Inj. :

Sigral Phase:

Signal Info:

\section{Response}

300000
280000 || 0419-16.DFIDIA

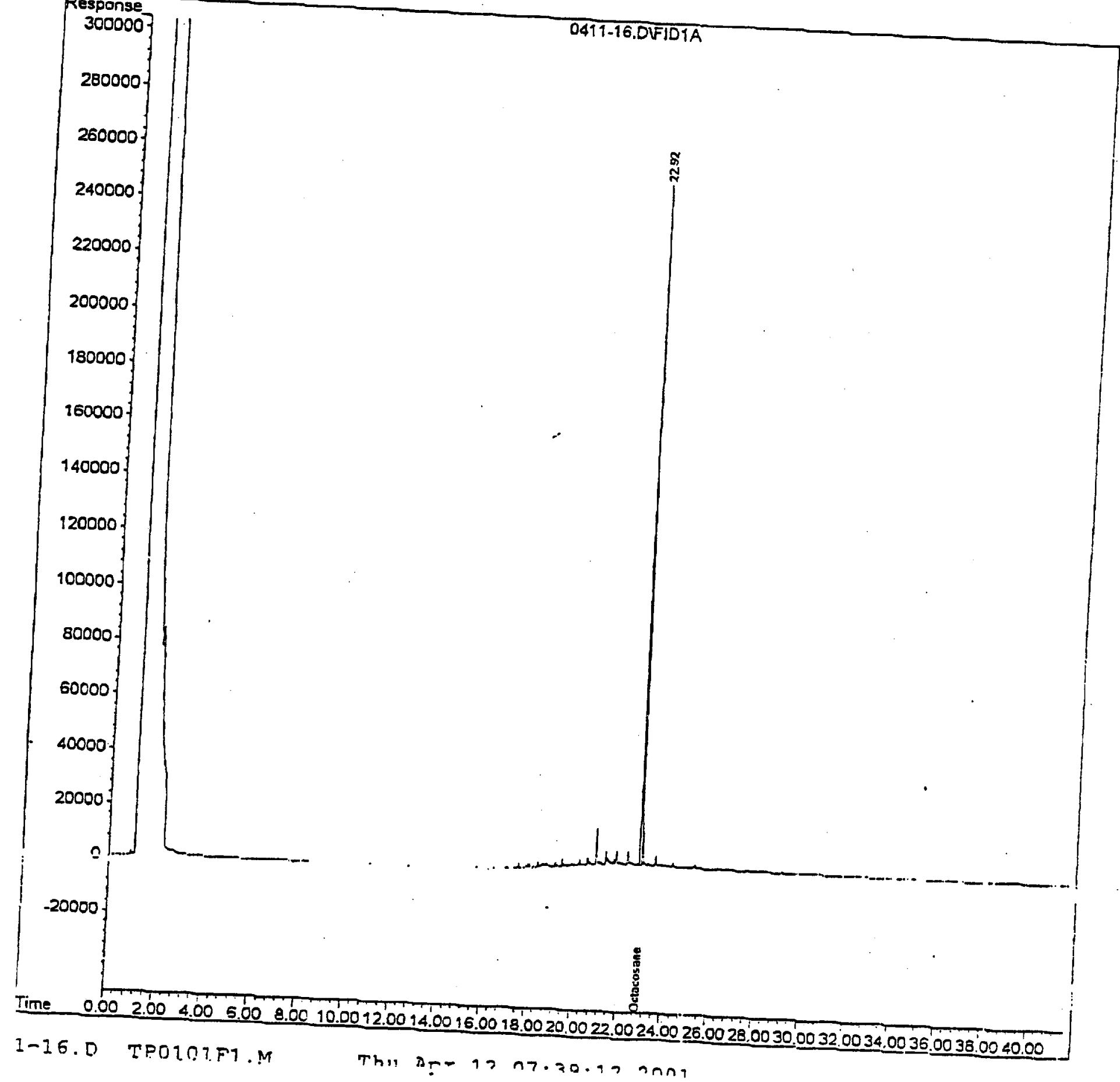

Operator: ccs

Inst : Seyrour

Mult:5Iะ: 1.00 
Quantitation Report

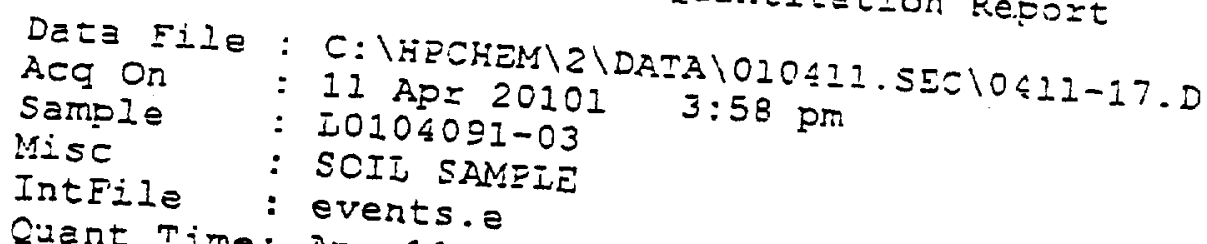

Quant Time: events.e

Quant Resuits File: IFOIO1R2.RES

Title

C: \HECHEM $2 \backslash M E T H O D S \backslash T P 0101 R 2 . M$

Last Update: 6/21/99 Seymour Eront TPH

Response via: MuItipled Jo 15:10:47 2001

Datakcq Meth: TP9907RI.M

Volume Inj.

Signal Phase:

Signal Info:

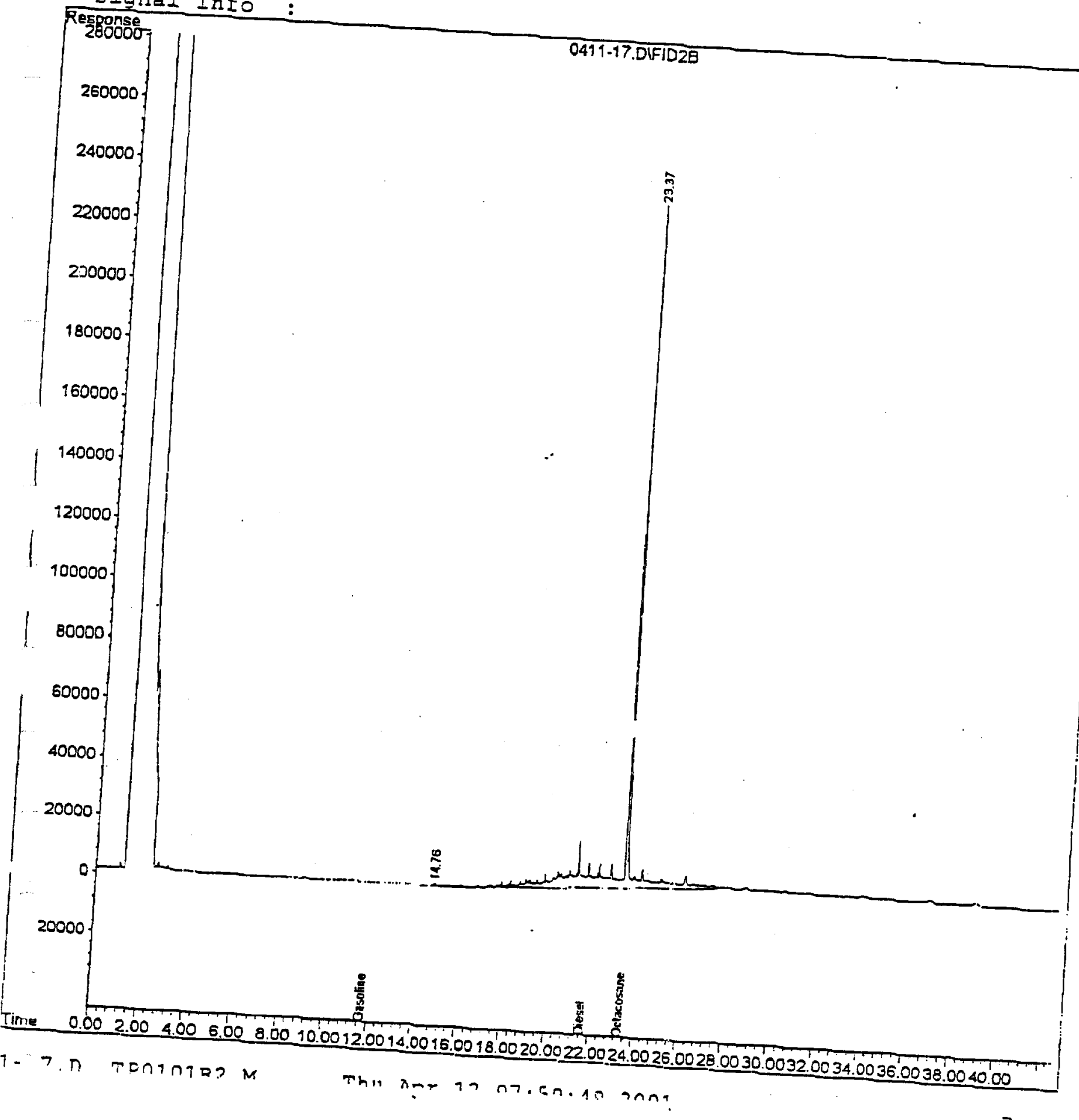


Quantitation Report

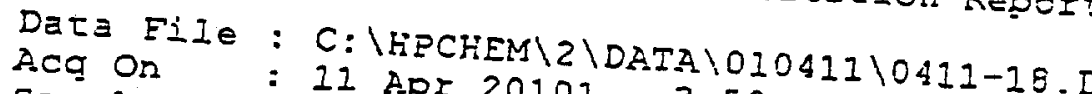

Sample : 11 Apr 20101 $10104091-04 \mathrm{pm}$

Mise :

Inteile: SOIL SAMPIE

Quant Time: events.e

API $11 \quad 16: 50 \quad 2001$

Quant Resuits File: TP0101E1.RES

Vial: 10

Opezator: CCS

Inst : sevmour

Multiple: $1 . \overline{00}$

Quant Metiod : C: IHPCHEM Z LMETHODS ITPOIOI

Title

: 6/21/99 SEMTOUT

Last Update: $F=i$ Jan Seymour Eront $T P H$

Response via: Multiple Ievel 51:13 2001

Datakcq Neth : TP9907Rl.M Lel Calibration

Volume Inj.

Signal Phase:

Signal Info

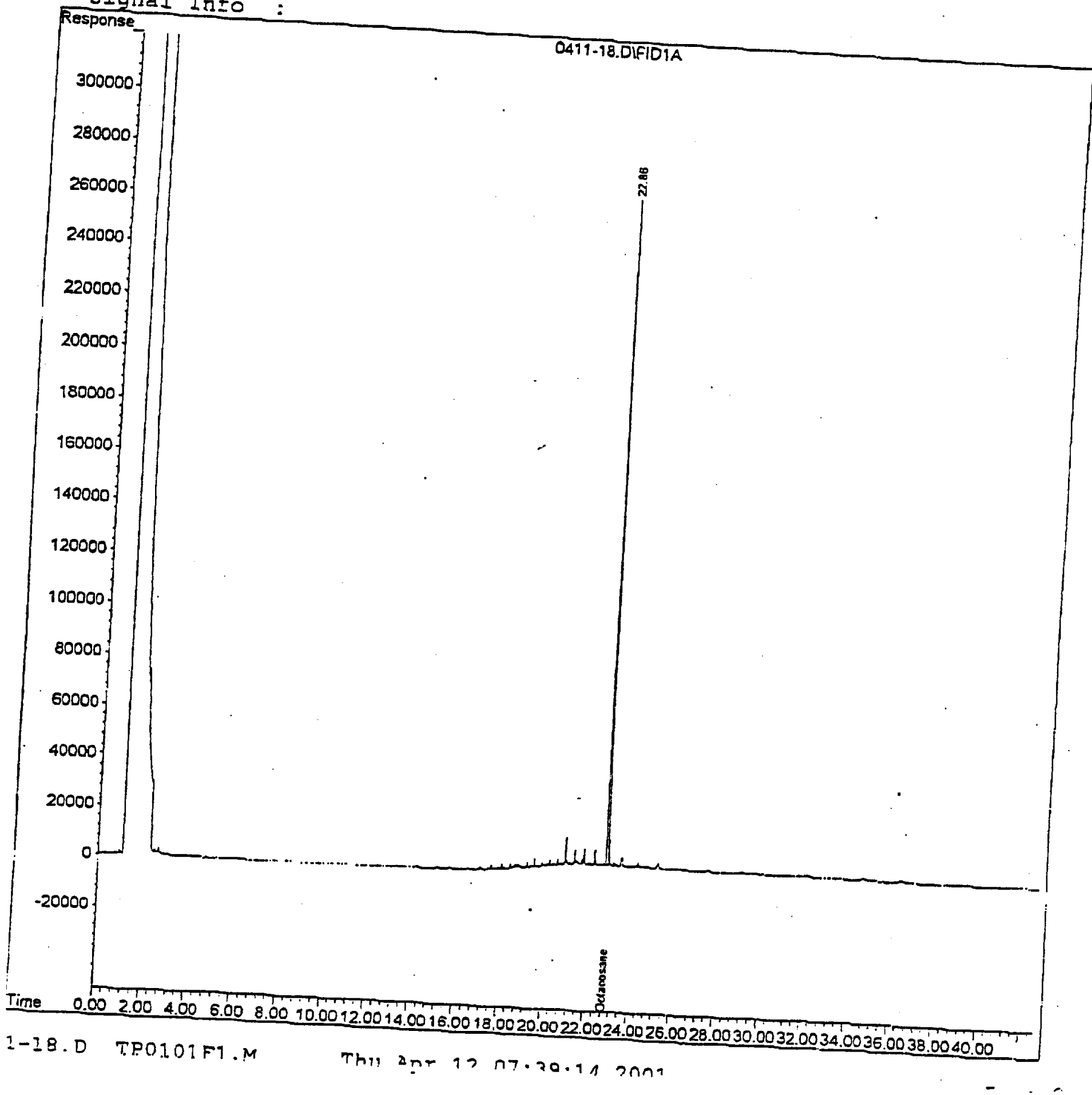


Quantitetion Report

Acq on

Sample

Mise

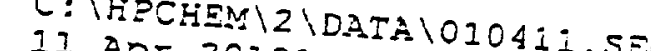

- 11 apz 20101

: 20104091-05

4:50 $\mathrm{pm}$

Vial: 12

IntFile: events

Quant Iime: :

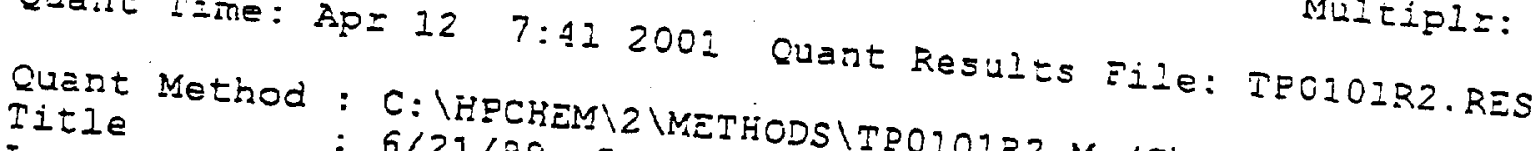

Last update: 6/21/99 seymour Eront TPH

Response via : Multiple Ievel Jan io 15:10:47 2001

DataAcq Meth : TPGgo7RI.M Lel Calibration

Operator: ciss

Inst

: Seytiour

MuLtipl=: 1.00

Volume Inj.

Signel Phase:

Signal Info

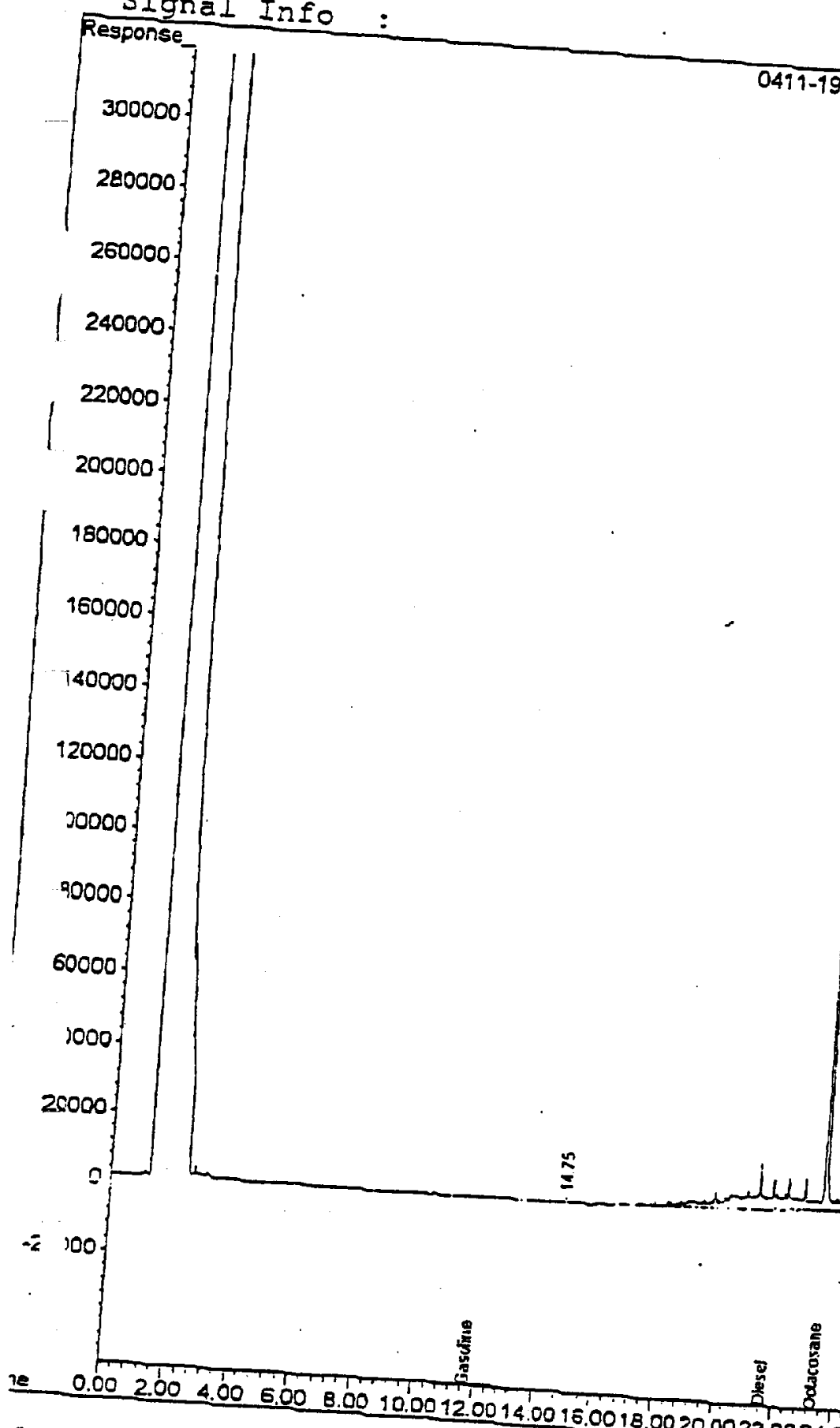

I? $\cap$ TFOTกTR?.M

Thil ?-- 1

กว. $5 n: 5$, ann 
$\begin{array}{lll}\text { Acg on } & : \text { II API ZOIOI } \\ \text { Sample } & : \text { I010409150 pm }\end{array}$

Misc : I0104091-06

IntEILE : SOIL SAMPIE

Quant Time: events.o

Quant Resules Eile: IP0101FI. RES

Tille

Lasi Update : EI/21/99 Seymour Eront TPH

Response via : Multiple Level $1: 13$ 2001

DataAcq Meth : TP9go7Rl.M Lel Calibration

Volume Inj.

Signal Phase:

Signal Inse :

Response

Vial: 12

Operator: cus

Inst

: Seymour

Muiciple: 1.00

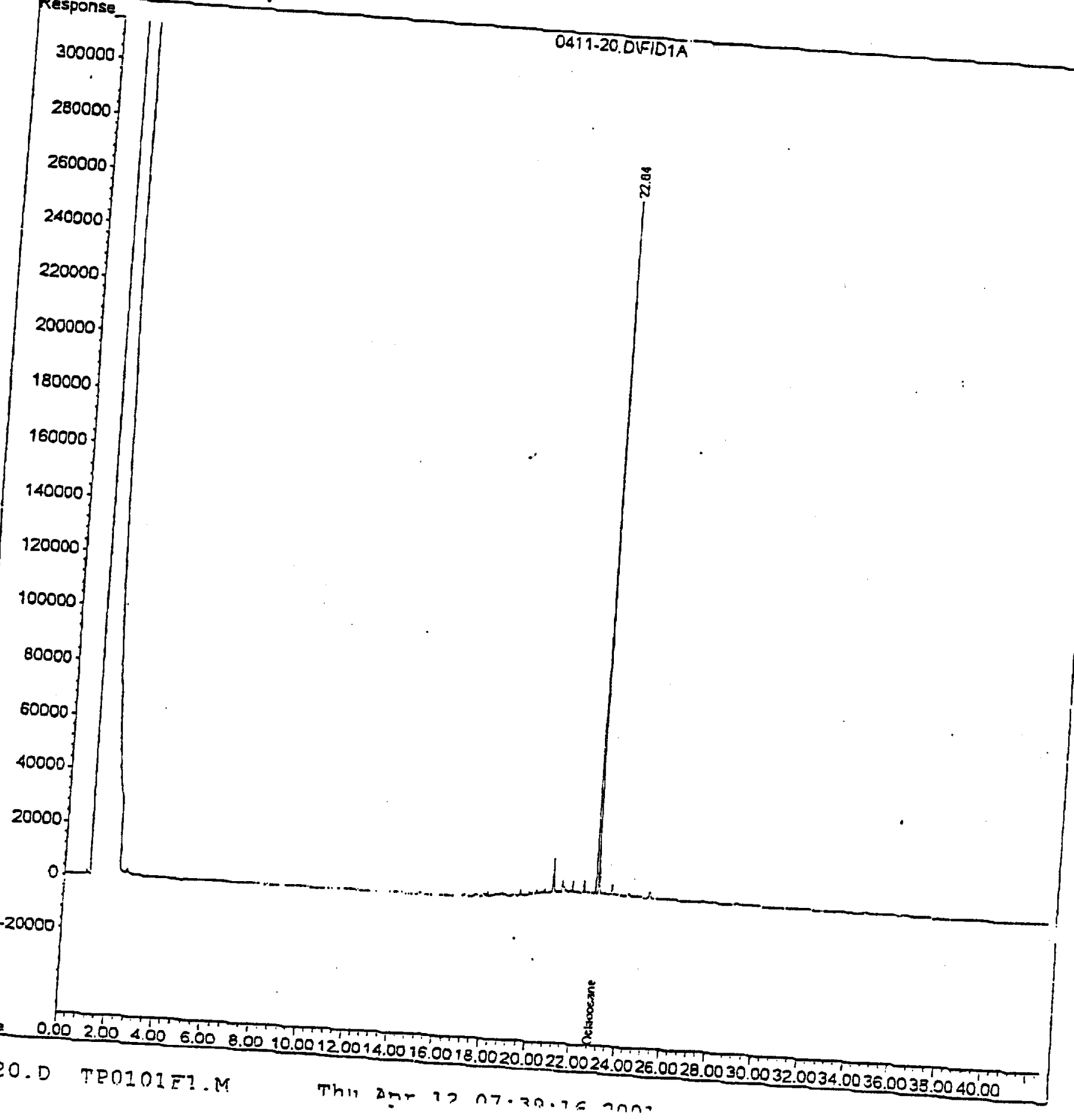


Data Eiqe

Quantitation Repo=t

$A \in q$ on

Sample

II API 20101

Misc

: I0104091-07

$5: 41 \mathrm{pm}$

Vial: I3

IntFile: SOII SAMELE

Quant Tins: events.e

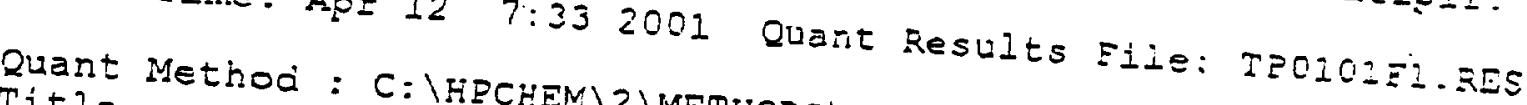

Title

Lest Update

: Eri Jan Seymour Eront TPH

Datanse via: Multiple Isvel Calib 2001

DataAcq Meth : TEg907Rl.M

Opezato: : cos

Inst

: Soymo:

Muitiple: i.00

Volume Inj.

Signal Phase:

Signal Info

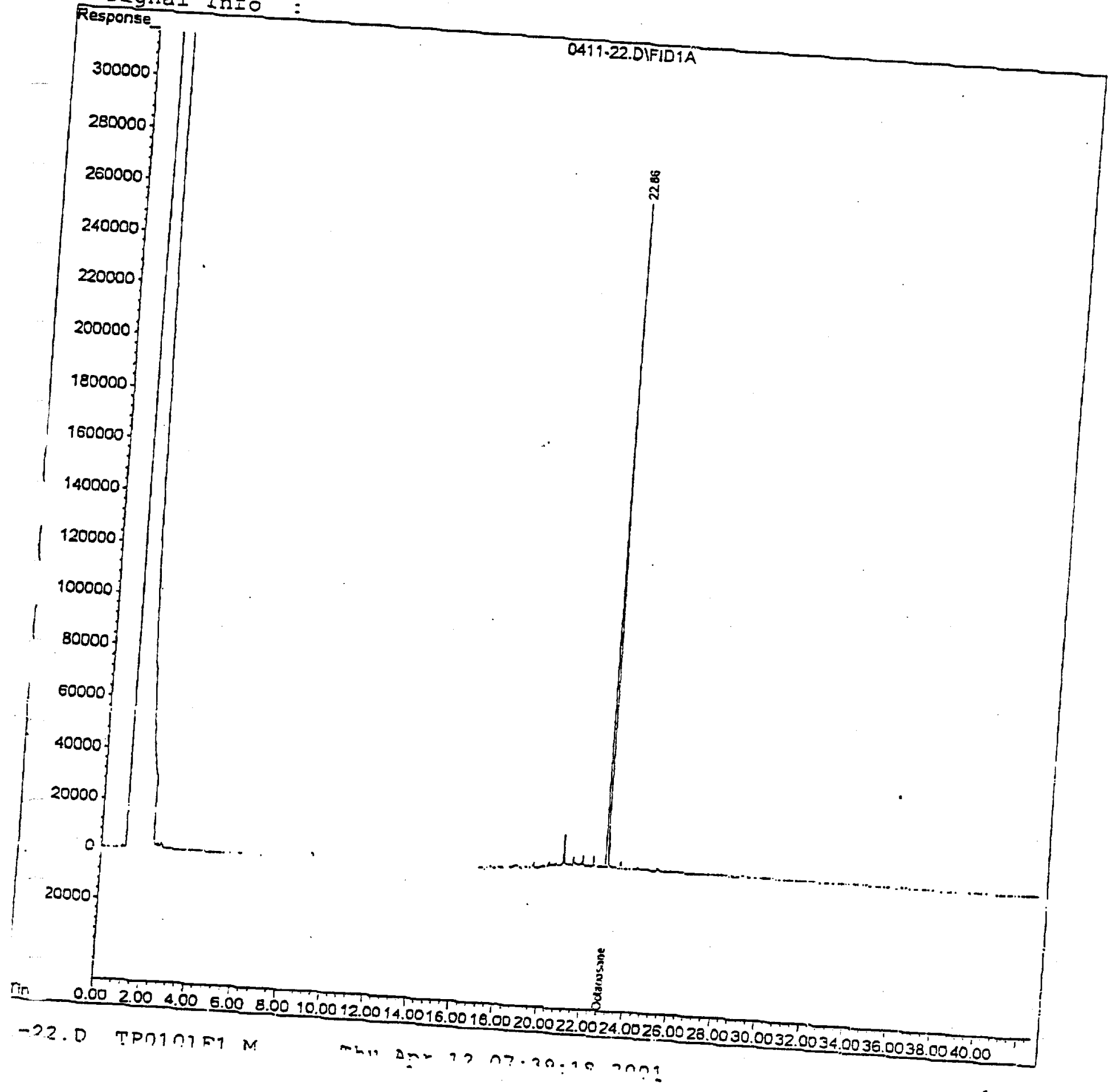


Acg On : 11 API 20101

Sample : L0104091-08 $6: 32 \mathrm{Fm}$

IntEIIE : SOIL SAMPLE

Viaz: 14

IntEIle: events.e

Quant Time: events.

Operatox: ics

hod $7: 342001$ Quant Results Eile: Tp0101Fl.REs

Title

Last Update: 6/21/99 Seymolir Front TPH

Irst : SEjmol:

MUItEDI : 1.00

Response via: Multiple5 08:51:13 2001

DataAcq Meth : Tp9907RI.M Level Calibratior

Volume Inj. :

Sigral Phase:

Signal Info:
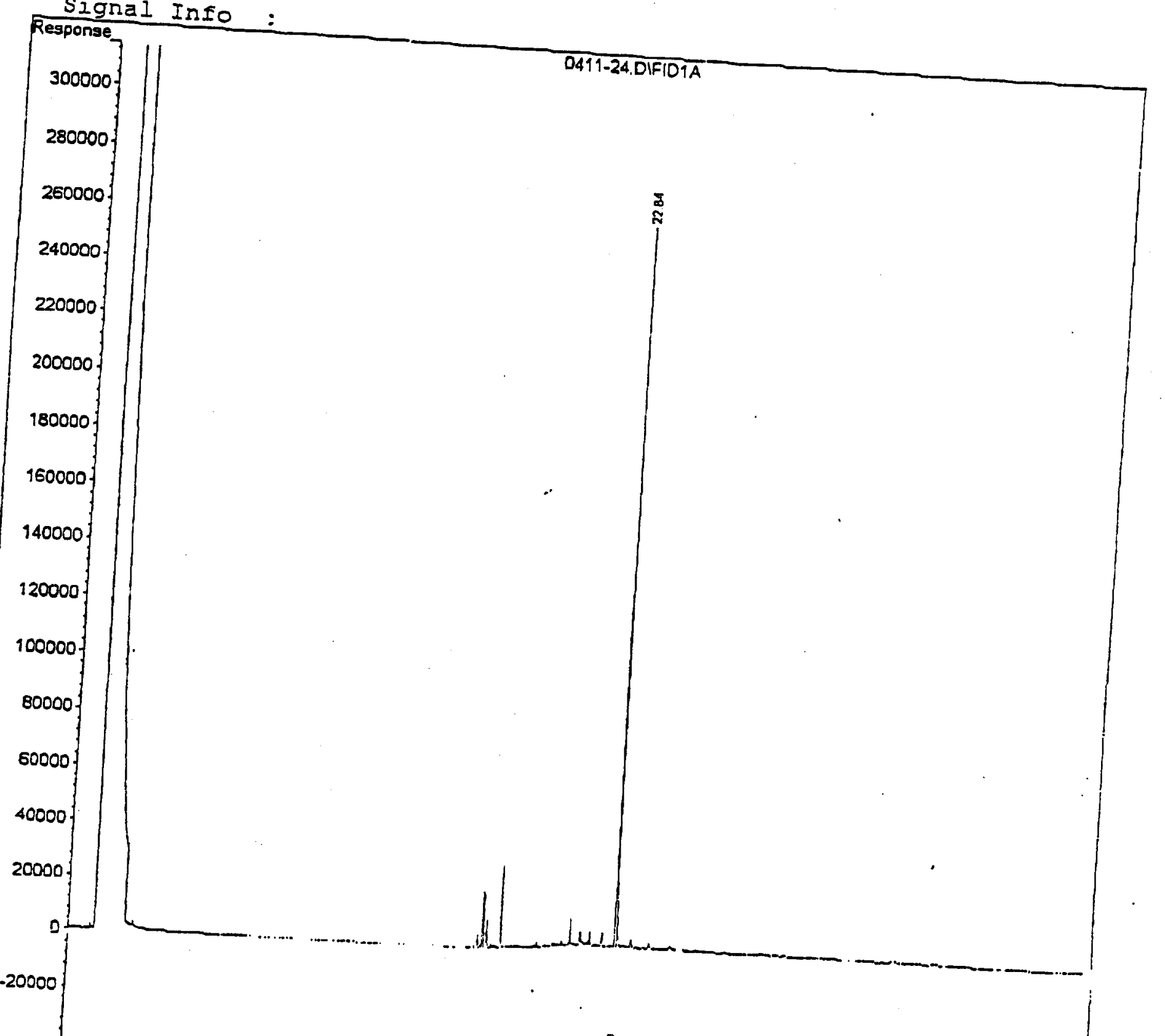

.... . in

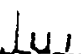

0411-24.DIFID1A

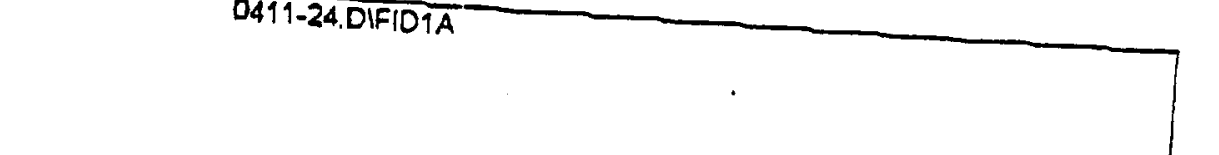

im

0.002 .004 .006 .008 .0010001200140019001800200022004

-24.D TP0101ET.M

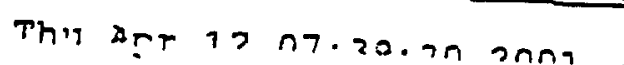




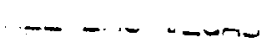

FAGE ID

Quartitation Report

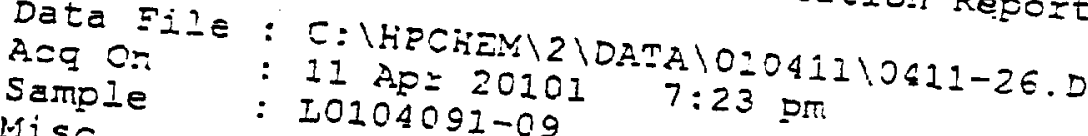

Mise

IntFile

Quant Time:

: SOIL SAMEIE

: events.e

Apr 12 7:35 2001

Quant Method :

Title

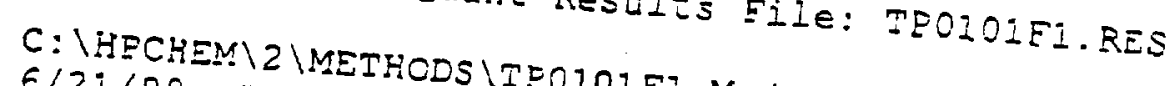

Iast Update : E/21/99 Seymour EIONT TPH

Response via: Mui Jan 05 08:51:13 2001

DataAcq Meth : TP9907Rl.M Lel Calibzation

Volume Inj.

Signal Phase:

Signal Info

Response :

$340000]$ 0411.26.DIFID1A

320000

300000

280000

260000

240000

1 .

200000

160000

140000

(

120000

100000

80000 .

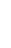

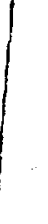

26.D TEO1OIEI.M 


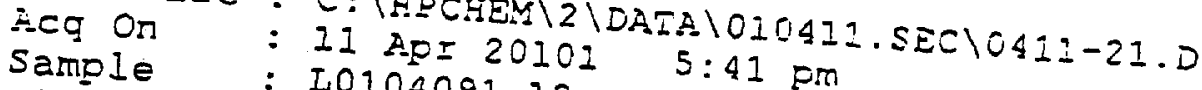

Misc

: I0104091-10

IntFile: SOIL SAMPLE

Quant Time: Ap= I2 7:43 2001

A 101041
$: 41$ pm

iaz: is

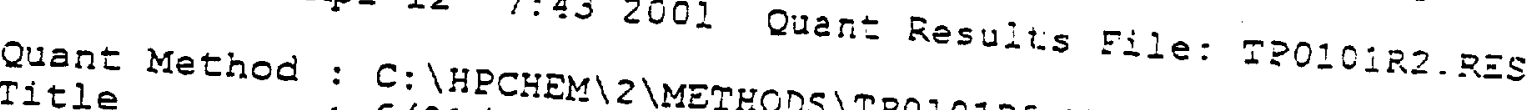

Opezator: CCS

Inst : Sejmou=

Multiple: 1.00

Last update

6/21/90

: Wed Jan 10 15: Front TPH

Response via: Multiple Ievel Calibration
DataAcq Meth: Tpg907Rl

IR9907RI.M

Volume Inj. :

Signal Phase:

Sigral Info:

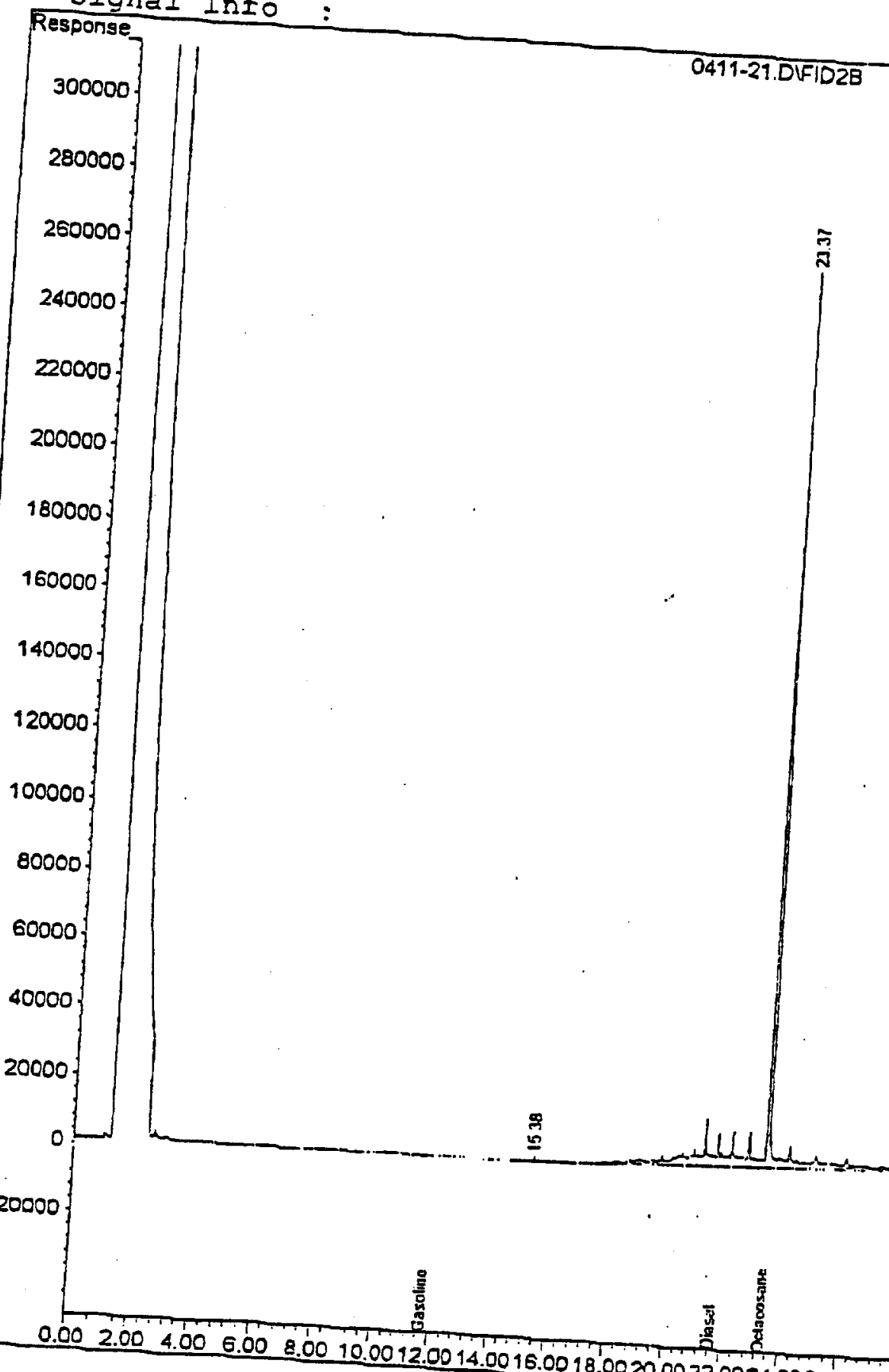

im

-2I.D TPOIOIR2.M

Thi drs 12 ก7.5n.E. Inn, 
Quantitation Report

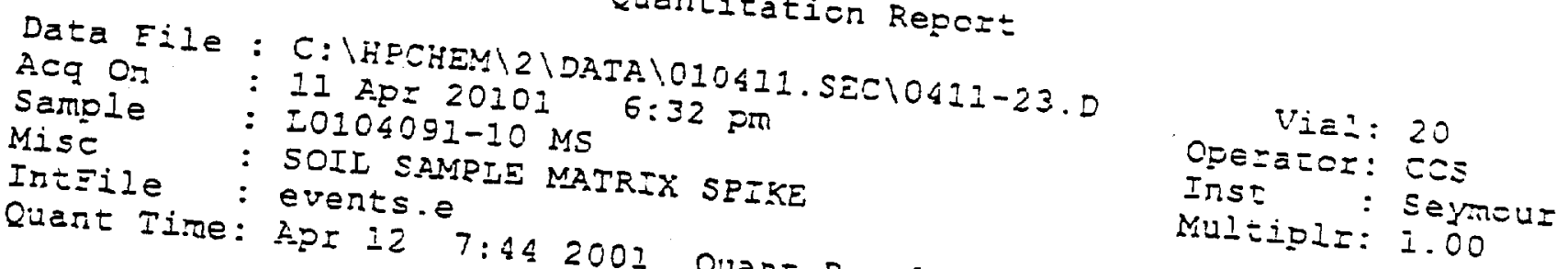

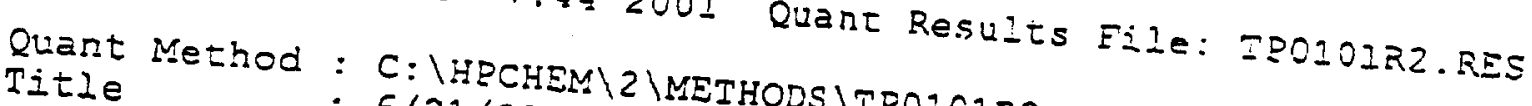

: 6/21/99M $2 \backslash M E T H O D S \backslash T P O 101 R 2 . M$ (Chemstation

Iast Update : Wed Jan 10 15:10:47 2001
Response via : Multiple Level Calibrot

DataAcq Meth: Teg9o7Rl.M Level Calibration

Volume Inj. :

Signal Phase:

Signal Info:

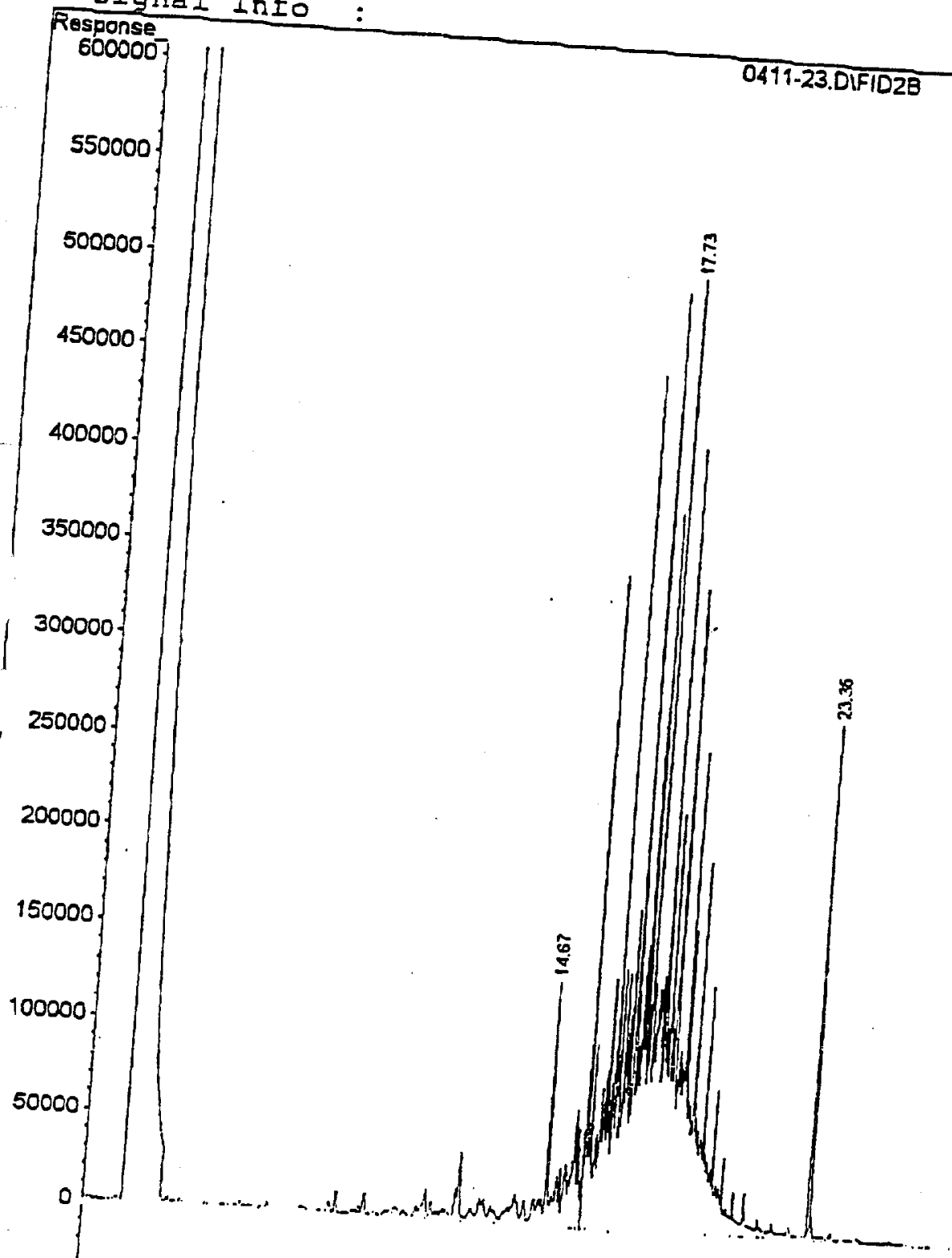

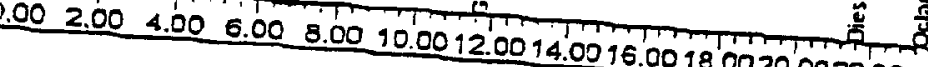

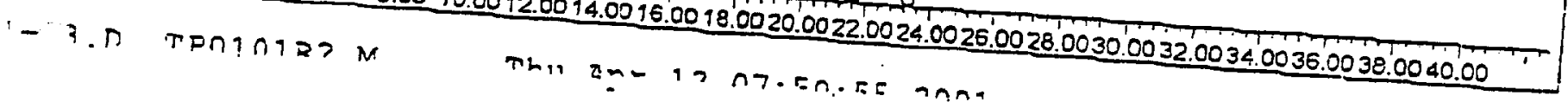


$\begin{array}{lll}\text { Acq on } & : \text { II ApI 20101 7:23 pm } \\ \text { Sampie } & : 20104091-10 & \end{array}$

Misc : L0104091-10 MSD

IntFile : SOII SAMPIE MATRIX SPIRE DUP

Quant Time: Apr 12

$7: 452001$

Quan: Result

Operitaz: 2i

ator: cos

Irist : Seymour

MLIEIPIS: 1.00

Title

Iast Update: Wed Jan 10 15:10:47 200I

DataAcq Meth : TP9907Rl.M

Volume Inj.

Signal Phase:

Signal Info

Response

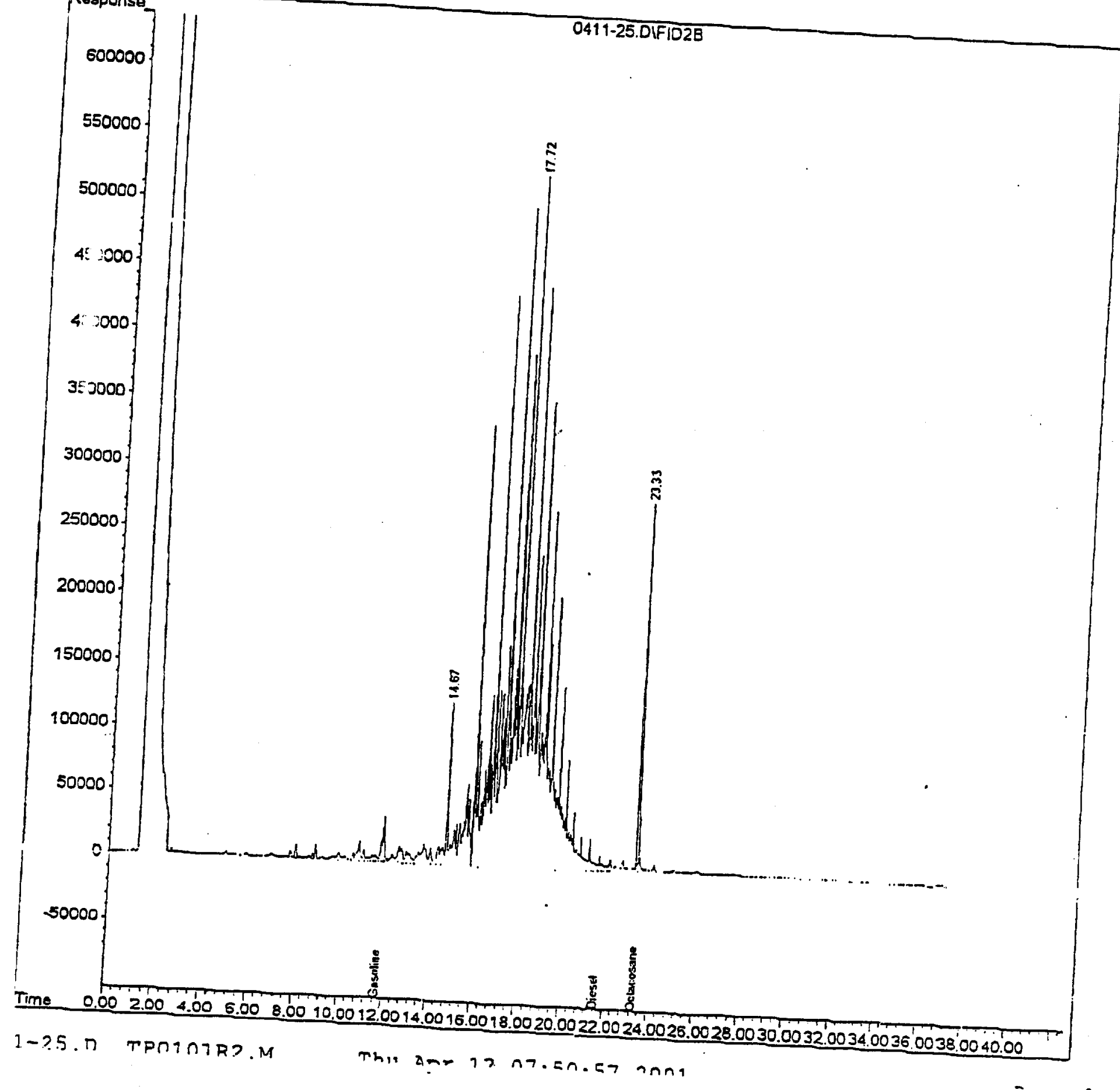




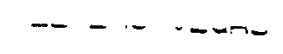

Quarititation Report

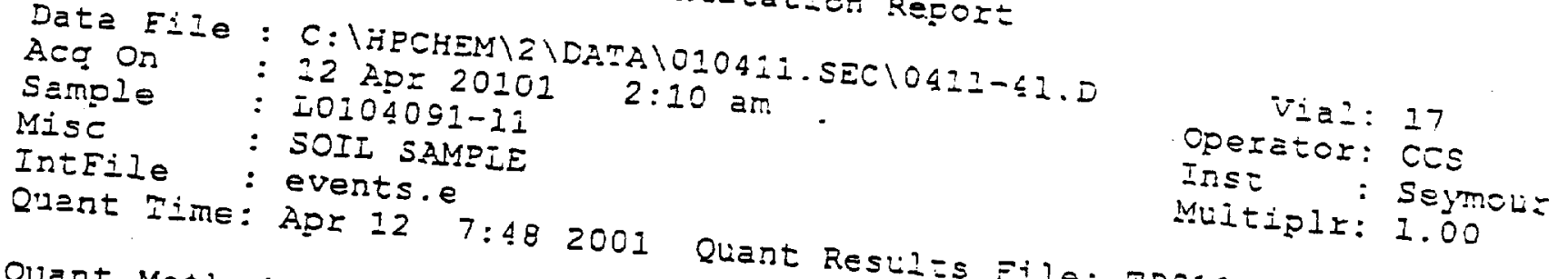

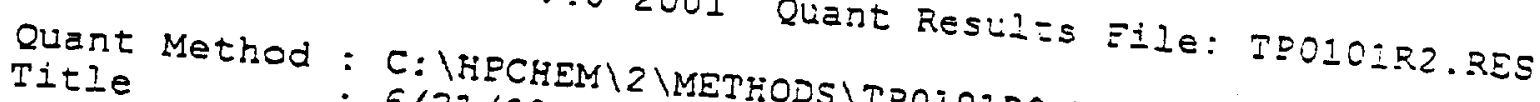

: $6 / 21 / 99$ SEYMOLI EVORT TPH

Response via: Wed Jan 10 15:10:17 2001

DataAcg Meth : Trg907Rl.M Ievel Calibiation

Volume Inj.

Signal Phase:

Signal Info:

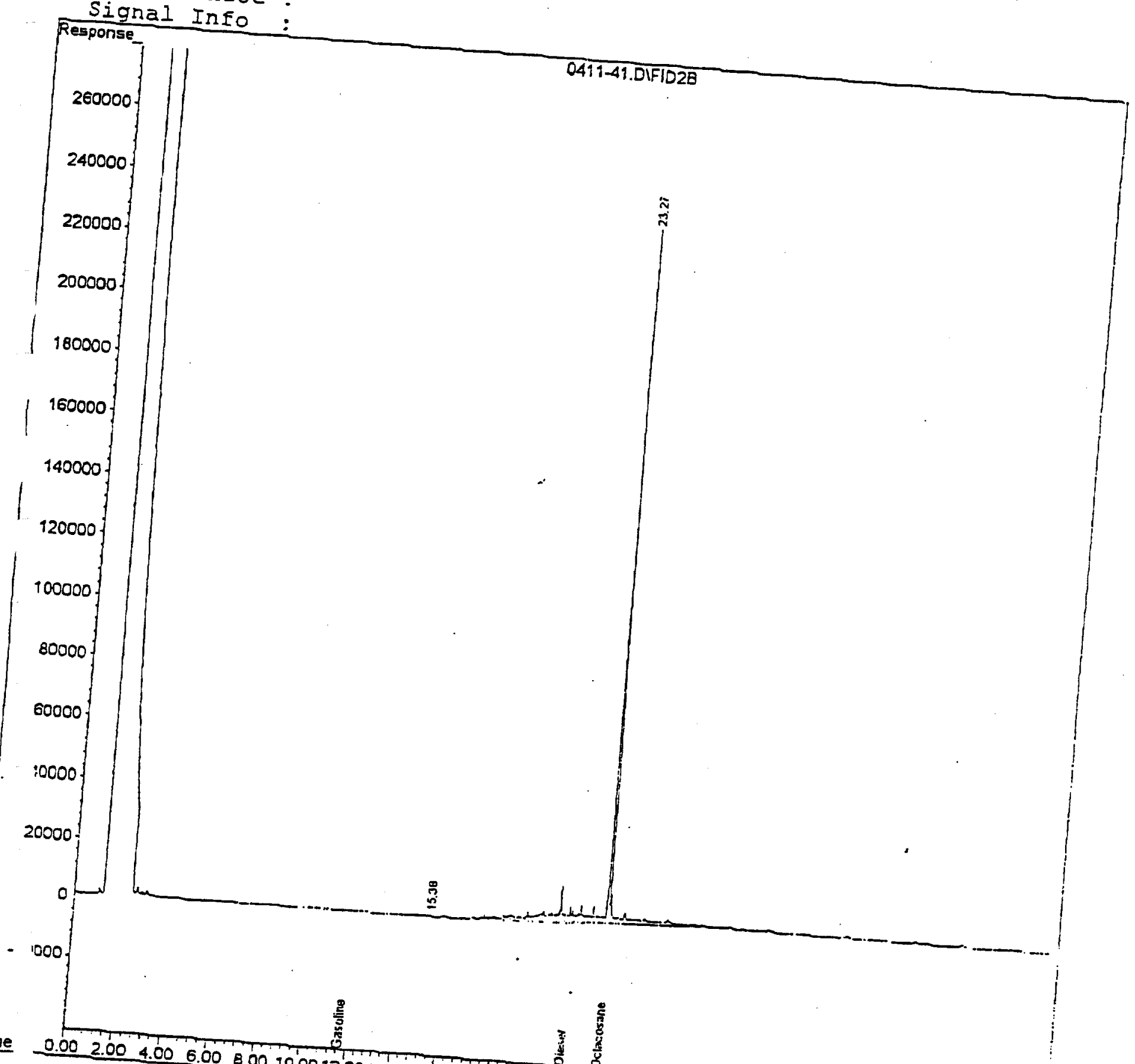




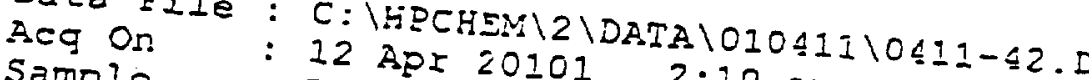

$\begin{array}{lll}\text { Sample } & : \text { I2 Apr 20101 2:10 ar } \\ \text { Misa } & : \text { SO104091-12 }\end{array}$

Mise

InTFile: SOII SAMEIE

Quant Time: events.e

Operial: Is

Opezator: CこS

Inst : Seinoir

Milipiz: 1.00

Quart Method :

Titie

: 6/21/99 Sejmour Eront TPH
Last Update : Eri Jan 05 08:51:13 2001

DataAcg Meth: Tpggo7Rl.M Level Calibration

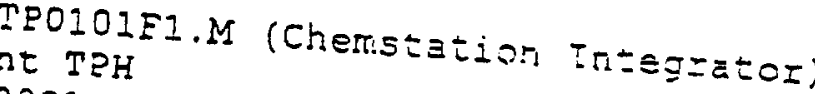

Volume Inj.

Signal Phase:

Signal Info

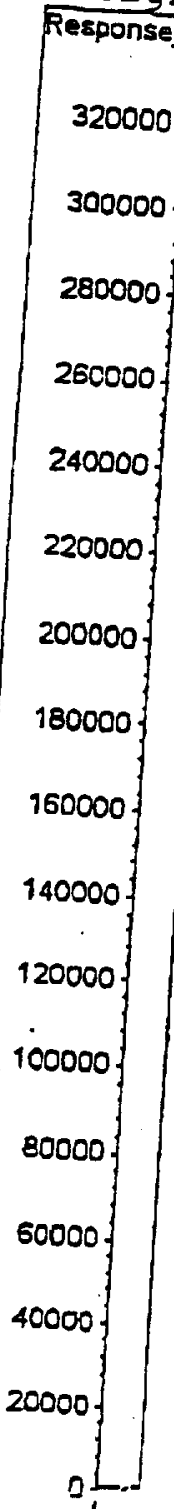

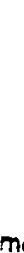

$-20000$

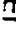

0.002 .004 .006 .008 .0010 .00120014

0411-42.DIFID1A

-42.D TPOTO1FT.M

Thil zme is ni.30.nc inna 
Uantization Rexort

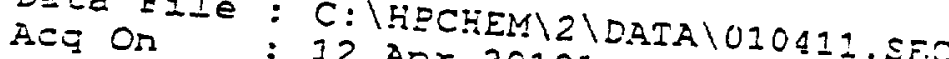

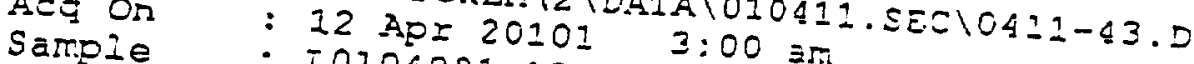

Misc : LO104091-13

IntFile : SOIL SAMPIE

Quazt Time: events.e

Title

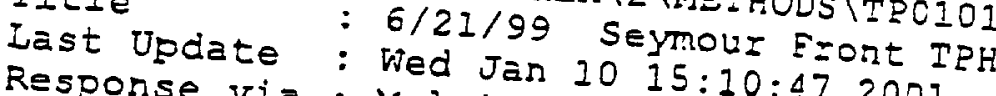

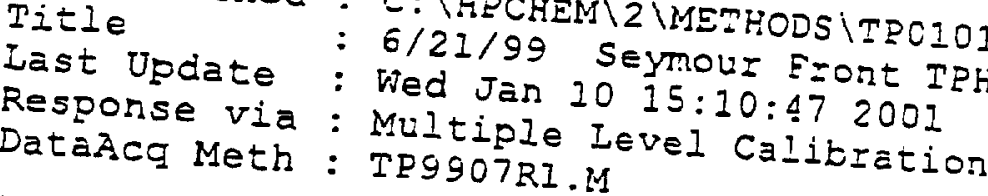

Volume Inj. :

Signal Phase:

Sigaal Info

\section{Response :}

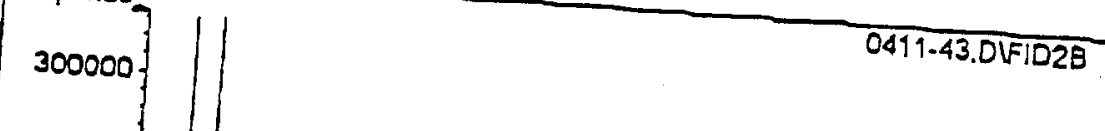

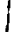

280000

240000

200000

160000

140000

19

1

100000

80000

\section{0}

20000

20000

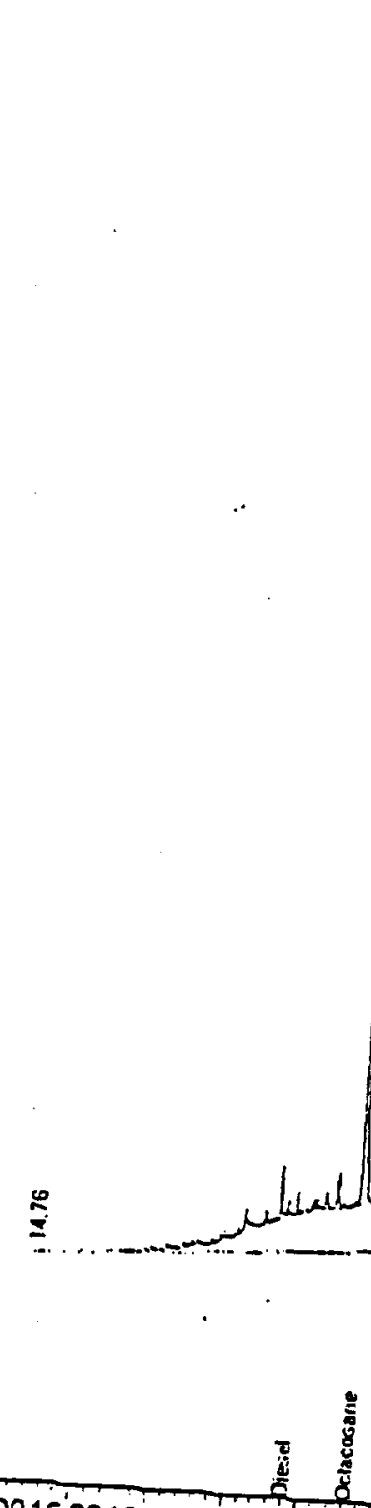

1 - ?.ก TDNIחIOO M

ลุ

opezator: is

Inst : Seymou=

lultip?r: 1.00 
Data File : C.lHPCHEM

ACG On : 12 API 20101 DAIA

Mampie : I0104091-13

IntEile : SOIL SAMPLE

Quant Time: events.e

Quant Method.

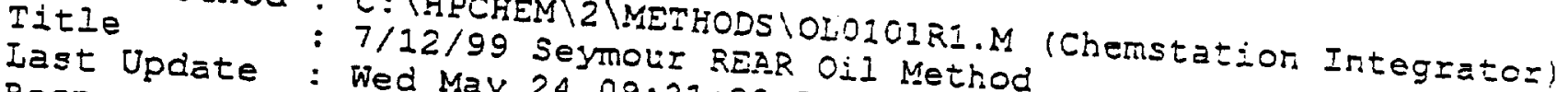

Response via: Med May 2409:21:09 2000

Datalcq Meth: TPSg07RI.M

Volume Inj.

Signal Phase:

Signal Info

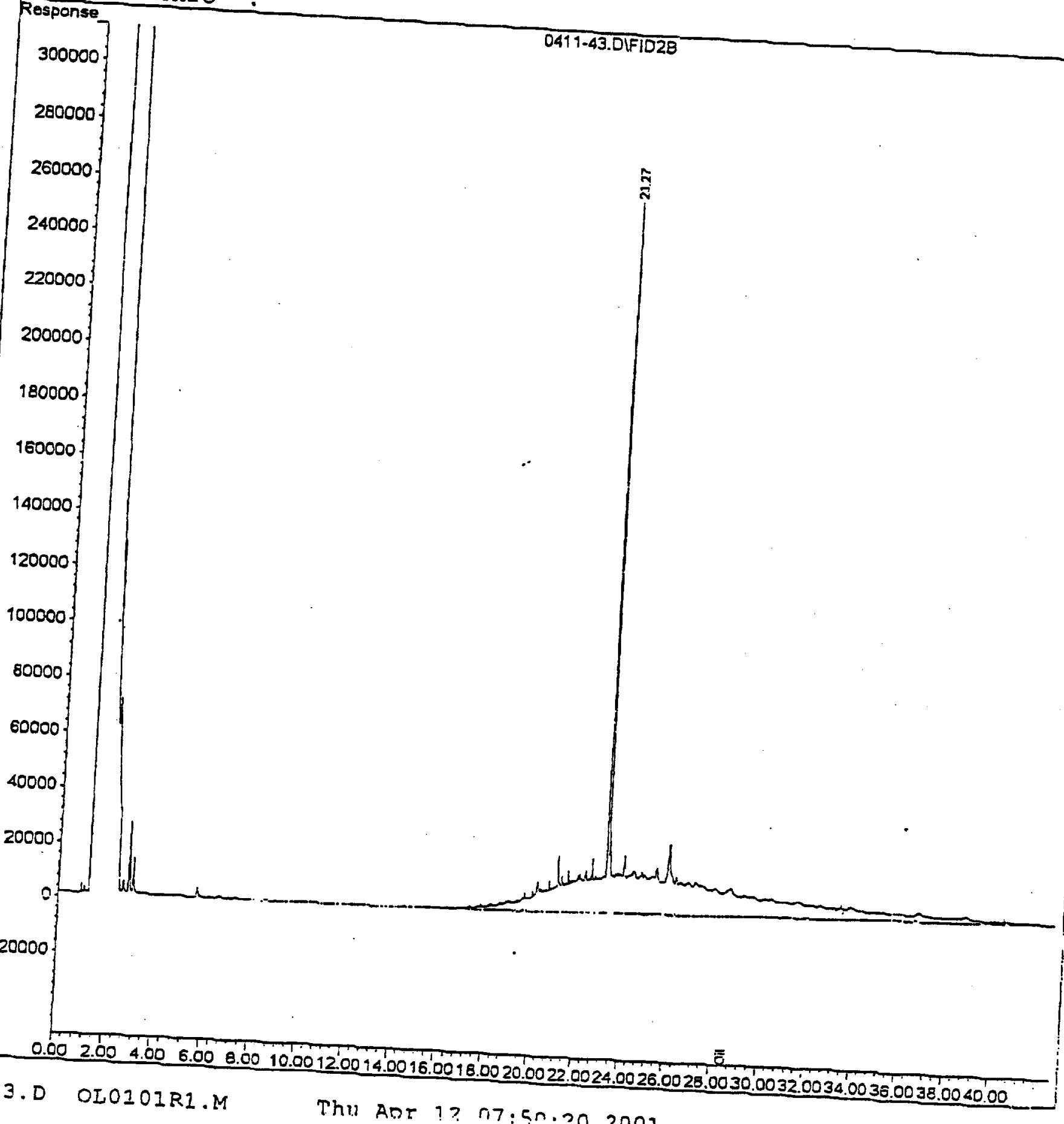

Operator: cos

Inst

: Seymouz

MUIEIPI: 1.00 
Data File : Quantitation Repozt
Aco on
Sample
Misc
i2 Apr 20101
I0104091-I4
IntFile: SOII SAMPLE
Quant Time: events.e
ApI 12 10:11 2001
$8: 05$ am
$2 \pi$.SEC $\ 0411-55.0$
Vißi: 25
Operacoz: cos
Inst: Seymola
MUItiFiz: I.00

Quant Results File: TP0101R2.REs

Titie

Last Update

Response via

: 6/21/99 SEYMOTHODS ITEO101R2.

via: Multiple Level 15:47 2001

DataAcq Meth : TP9907RI.M Lel Calibration

Volume Inj.

Signal Phase:

$\frac{\text { Signal Info : }}{\text { Response }}$
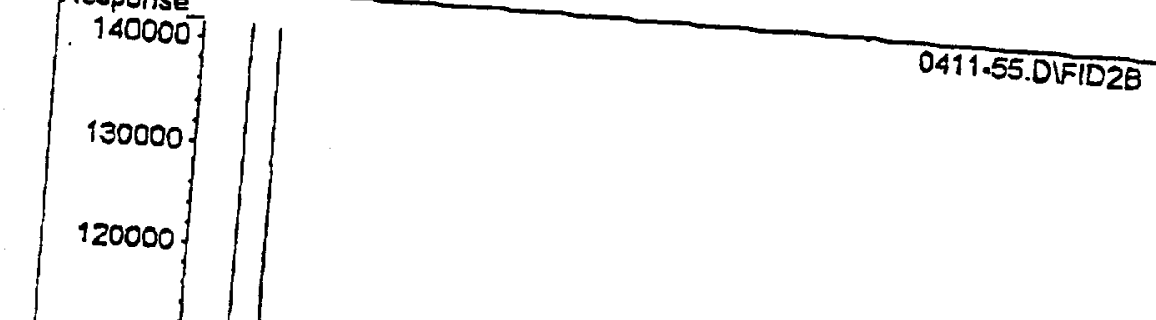
Quartitation Report

Data File : C: \HECFEM z\Data

Acg on

Sample

: 12 Apr 20101 8:05 $0111.5 E 010411-53.0$

Misc

: $20104091-14$

IntEIle: SOIL SAMPIE

Quant Iime: events.e

Ine: Apr 12 10:12 2001

Quant Method

Title

C: \HPCHEM $\backslash 2 \backslash M E T H O D S \backslash O I 0101 R$ i

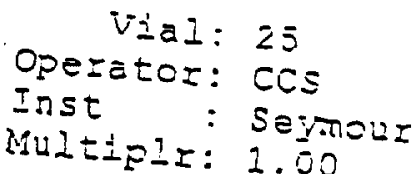

Inst

1.00

Last Update : 7/12/99 Seymour REAR Oil Method

Response via: Wed May 24 09:21:09 2000

DataAcq Meth: TP9907RI M

Volume Inj.

Signal phase:

Sigral Info
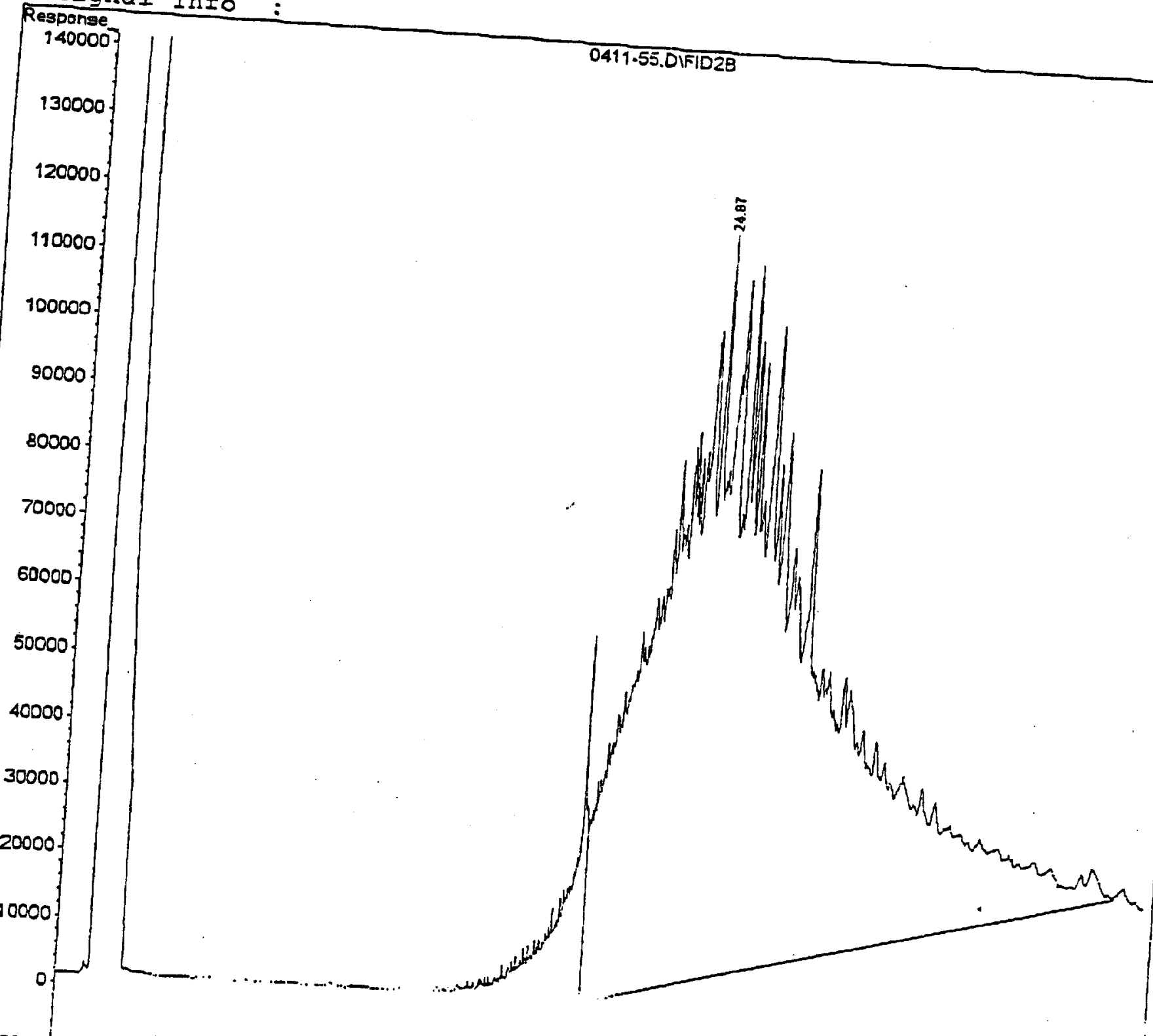

$-10000$

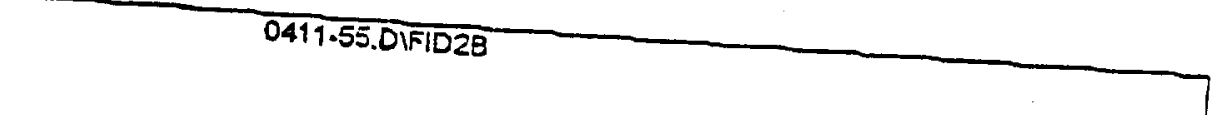

- GE $n$ กT.กIก?D1. M

5 
Data File. Quantitation Report

Acg on : i2 APCHEM ADIDATA $201010411 \backslash 0411-56.0$

Misc : L0104091-15 $8: 05 \mathrm{am}$ in

IntEile: SOIL SAMPIE

Quant Time: events.e

: Apr $1210: 172001$ Quant Resulis Sile: TP0101El.REs

Title

Last Update: $6 / 21 / 99$ Seymour Front TPH

Response via: Multiple 05 08:51:13 2001

DataAcq Meth : TP9907RI.M Lel Calibration

Volume Inj.

Sigral Phase:

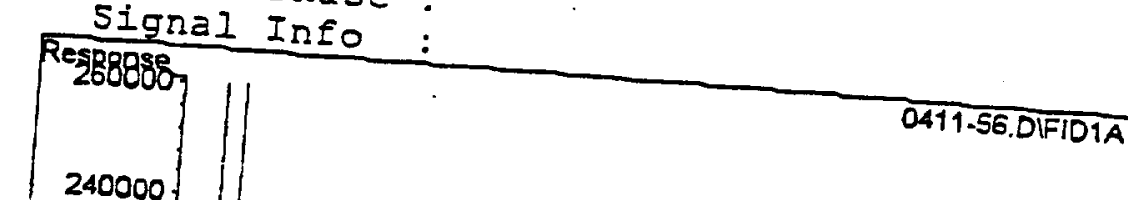


Quantization Report

Acq on : I2 ApY 20101 A

Sample : L0104091-15 8:05 am

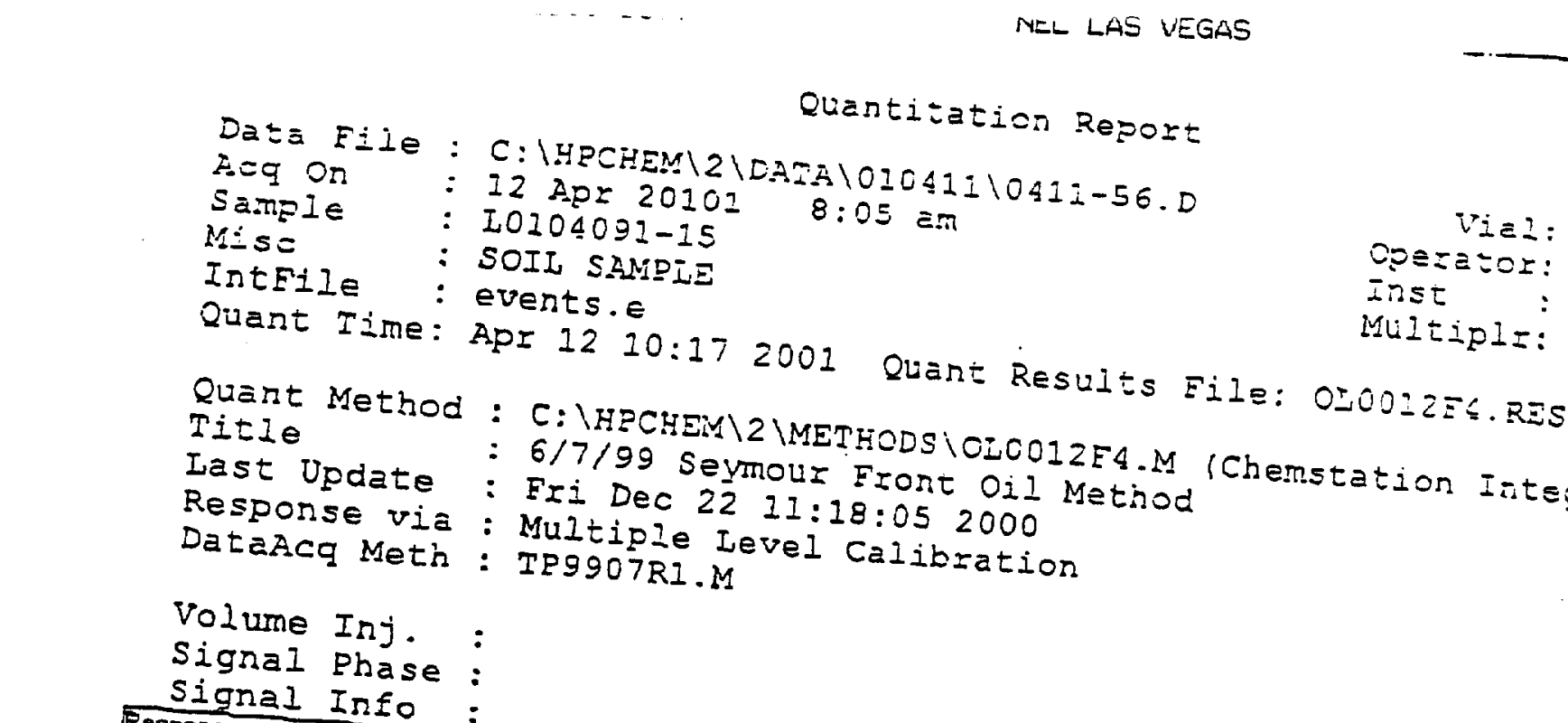

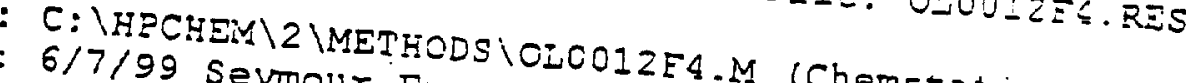

Last Update: $6 / 7 / 99$ Seymour Front Oil Metiod (Chemstation Integrator)

Response via: Fri Dec 22 II:18:05 2000

DataAcq Meth: Trg9o7Rl.M Lel Calibration

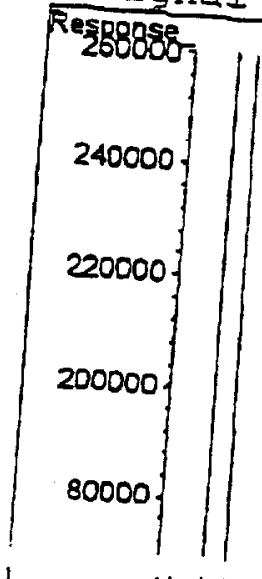

\begin{tabular}{|}
160000 \\
140000 \\
120000 \\
100000 \\
80000 \\
60000 \\
40000
\end{tabular} \mid
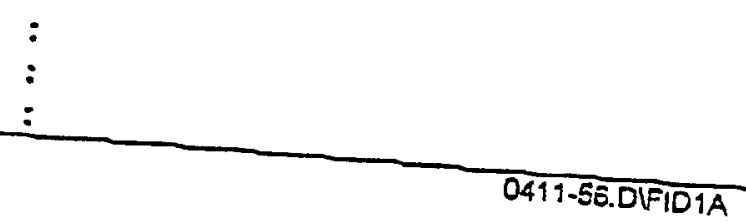

0417-55.DFID1A

$-20000$

000
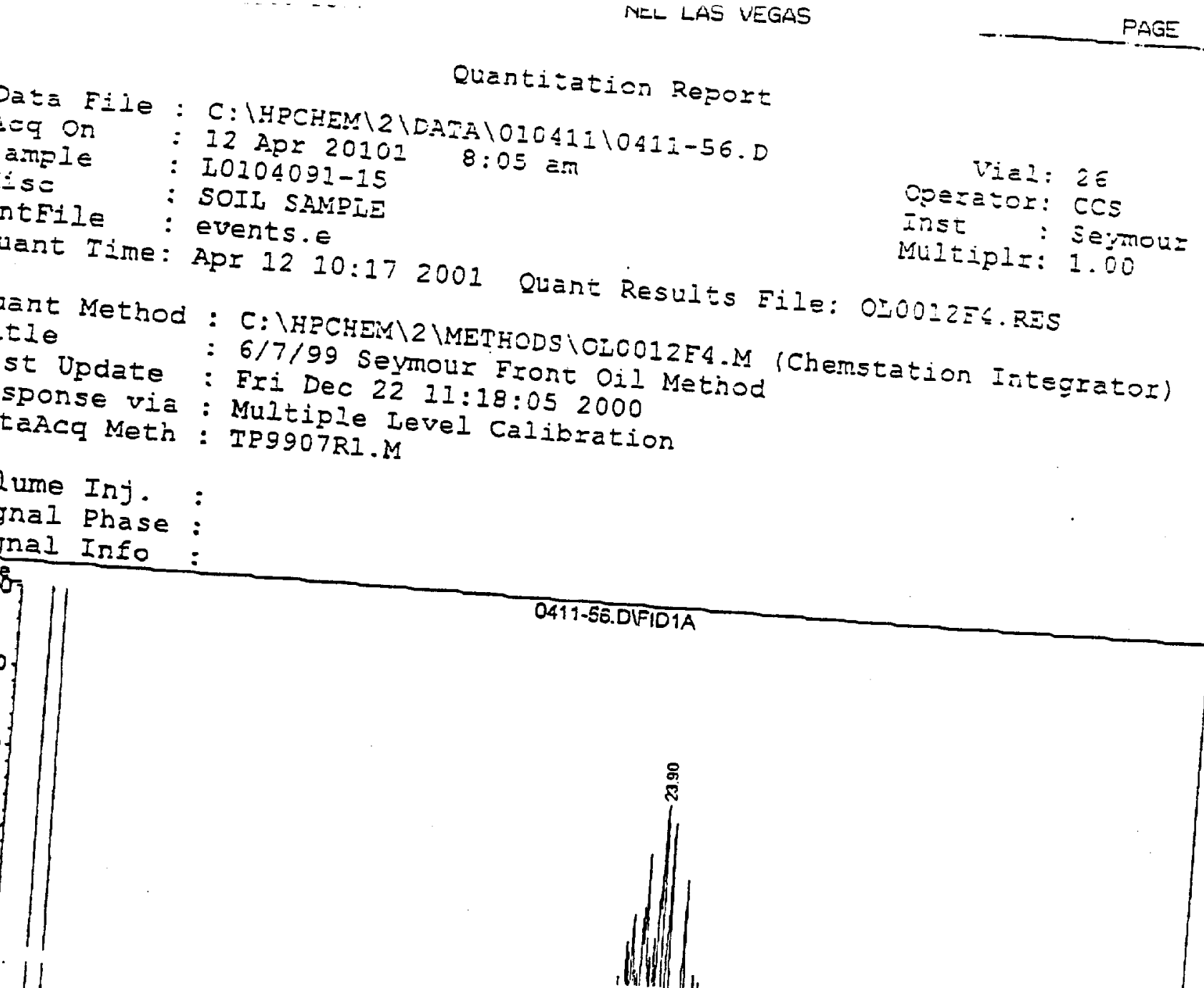

$$
\begin{aligned}
& \begin{array}{l}
\text { Opazazor: cCs } \\
\text { Inst : Seymour } \\
\text { Multipla: 1.00 }
\end{array} \\
& \begin{array}{l}
\text { Opazasor: CCs } \\
\text { Inst : इeymou= } \\
\text { MLItipl=: } 1.00
\end{array}
\end{aligned}
$$

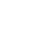

$$
\begin{aligned}
& \text { Quantizat } \\
& \text { IA\0I041I } \\
& \text { 8:05 en }
\end{aligned}
$$
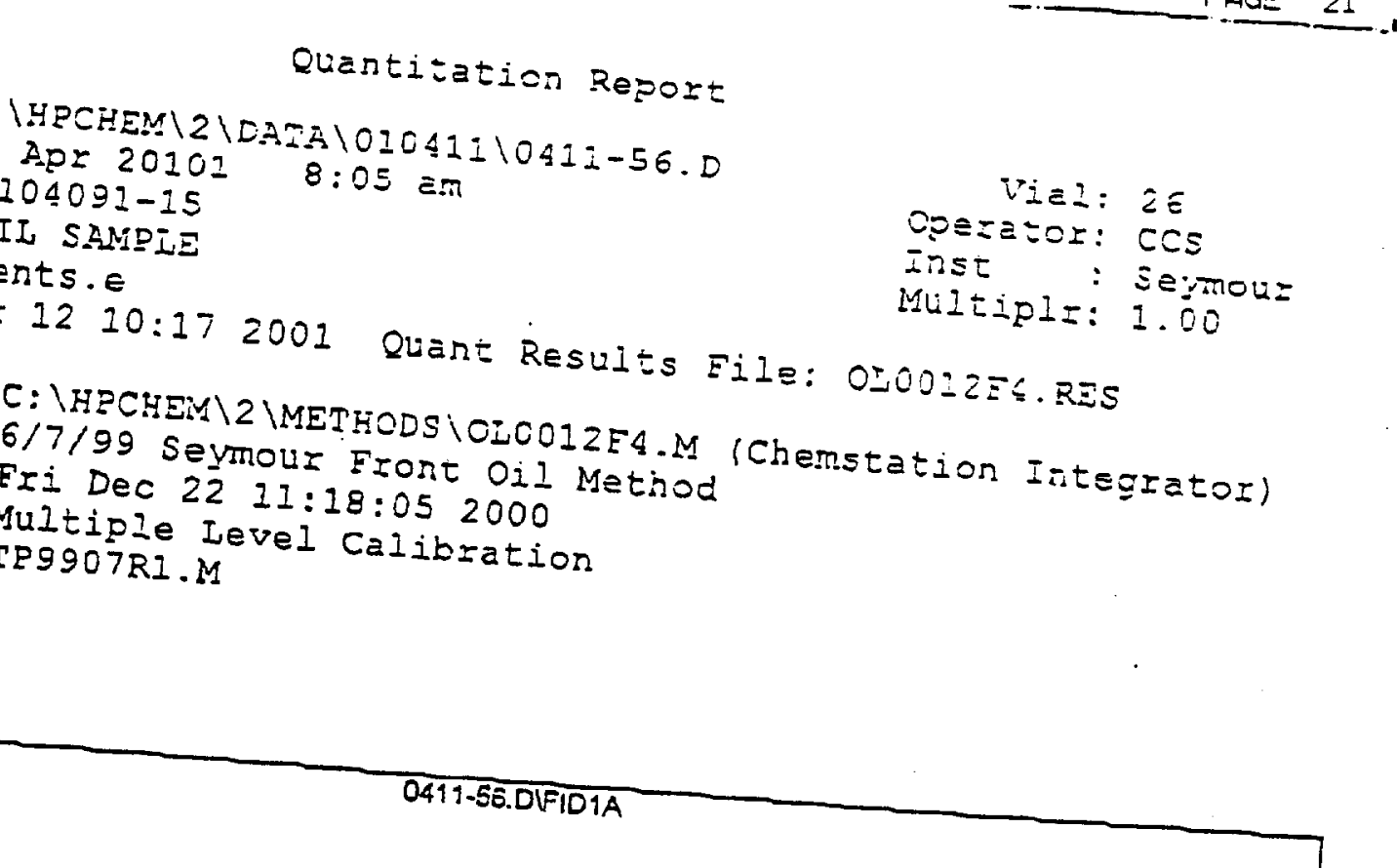

. -5S.D 


$$
\text { PROJECTICLIENT IMFOAMAMIION }
$$

\begin{tabular}{|c|c|}
\hline Proplect $C A u \geq 30 / 320$ & aN Orgh: 2,50 \\
\hline$C>\Delta 19 \Delta 61$ & ASL Prog:: \\
\hline
\end{tabular}

Send Repor w: $\triangle Q N$ TOBIASON

Thone:295-6/69.|702-295-7761 $\left.\right|^{\mathrm{Fac}}$

Proloct Manager: WA T Y NE JOHNS ON

. Tumaround: (1) Slandard - 30 days Non rad, on Days Rad, Other:

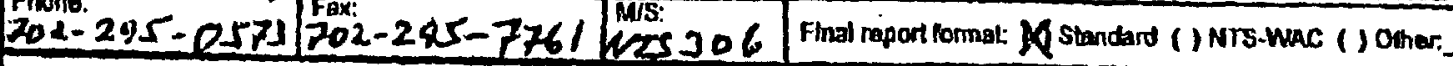
SAMPLE INFORUATION

Samping Sller AU 310 SLUDGE BED The samples subritted contin (clieck): f. I Haxurdour (I Radlasellive contamination. MU Unknown

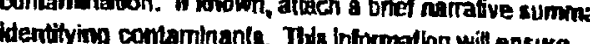

compliance with applicable requlations and allow tow tro ate
handing of the sample matelals.

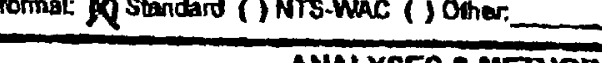
Rad SGO: LAB USE OMLY

\begin{tabular}{l|l} 
Rad SGD: & Non-Rad SDG: $V / 059$ \\
\hline Rad Pachet & Mon-Rad Packet:
\end{tabular}

Cuent Services Representative:

Whi these analyses bo perlomed undar a shoned SOWr? (I YES () NO Yso, do analysas entered here agree with tho sOWn (IVES () NO ( )NA If not, Identity the variation Dale:

CSR inthals indlcating review and approvat:

9
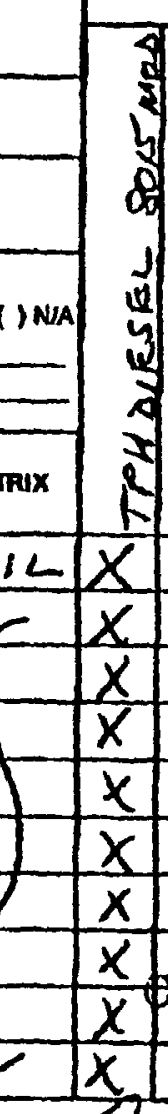

$x$

Inansfer of semplas submilted for analyses

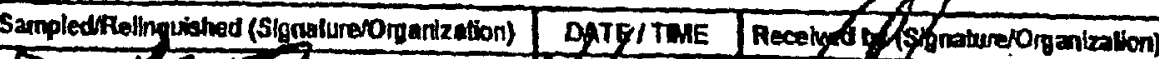

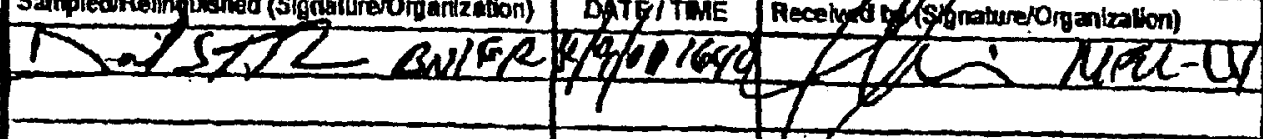



Complete for samples shipped bo un OfF-81TE Subcontrace Laborator

Relinqulehed (BN Representotive Signature)

\begin{tabular}{l} 
Rolinqulahed (Courfor \& Tracting Info) \\
\hline Relinqulahed (In Mer Subcontractor Rep)
\end{tabular}


PRONECTICLIENT INFORMATION

\begin{tabular}{|c|c|}
\hline Projectcay 2.30/320 & BNOrgt: $2 / 50$ \\
\hline Chargo No: $C 7019 \Delta 61$ & Ast Prog: \\
\hline
\end{tabular}

C010409/Page z of 2 REPORT UFFRIMTION

Profect Manager. WAYNE JeHNSON

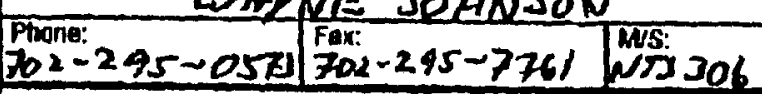
Send Repor w: $\triangle A N$ TOBIASON PDon: 295-6/69 ||$_{7022}^{\text {fax }}-295-\left.7761\right|^{\text {WS: NTS } 306}$ Tumaund: () Standard - 30 days Non-rag. 60 Days Rad, Other

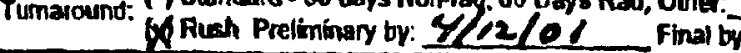
SAMPLE INFORAMATION

sampling slie CAU 320 SLLOGE BED The somples submillad contain (chect):

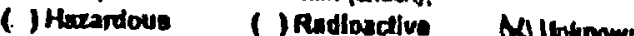
contamination. I known, attach a briet narrative esmmary contaminalon. I known, allach a bries narrathe summary Idenlifying Domlaminanle. This imioimation will enswe Final report format Wo standand () NTS WuC ( ) Other. compltance with applleable regutations and allow for the sate

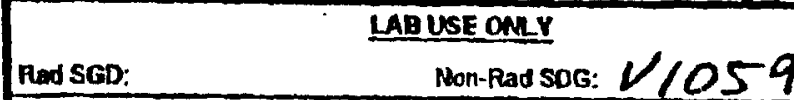

\begin{tabular}{l|l}
\hline Rad Packet & Non-Rad Packel: \\
\hline
\end{tabular}

Cllem Services Representative:

Wult these analyses bo pertomed under a algned sOW7 () YES () MO If sa, do analysea entered hero agnee with the SOWn (IYES (INO (INAA H noh, bertisy the variation

CSA bullats indicaling review and approval: ___ Date:

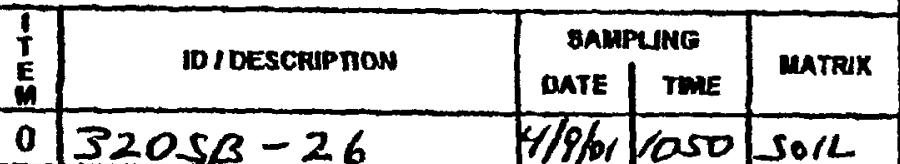

$1213205 B-27$

$13232090-28$

M 3 32058-29

$1543205 B-30$
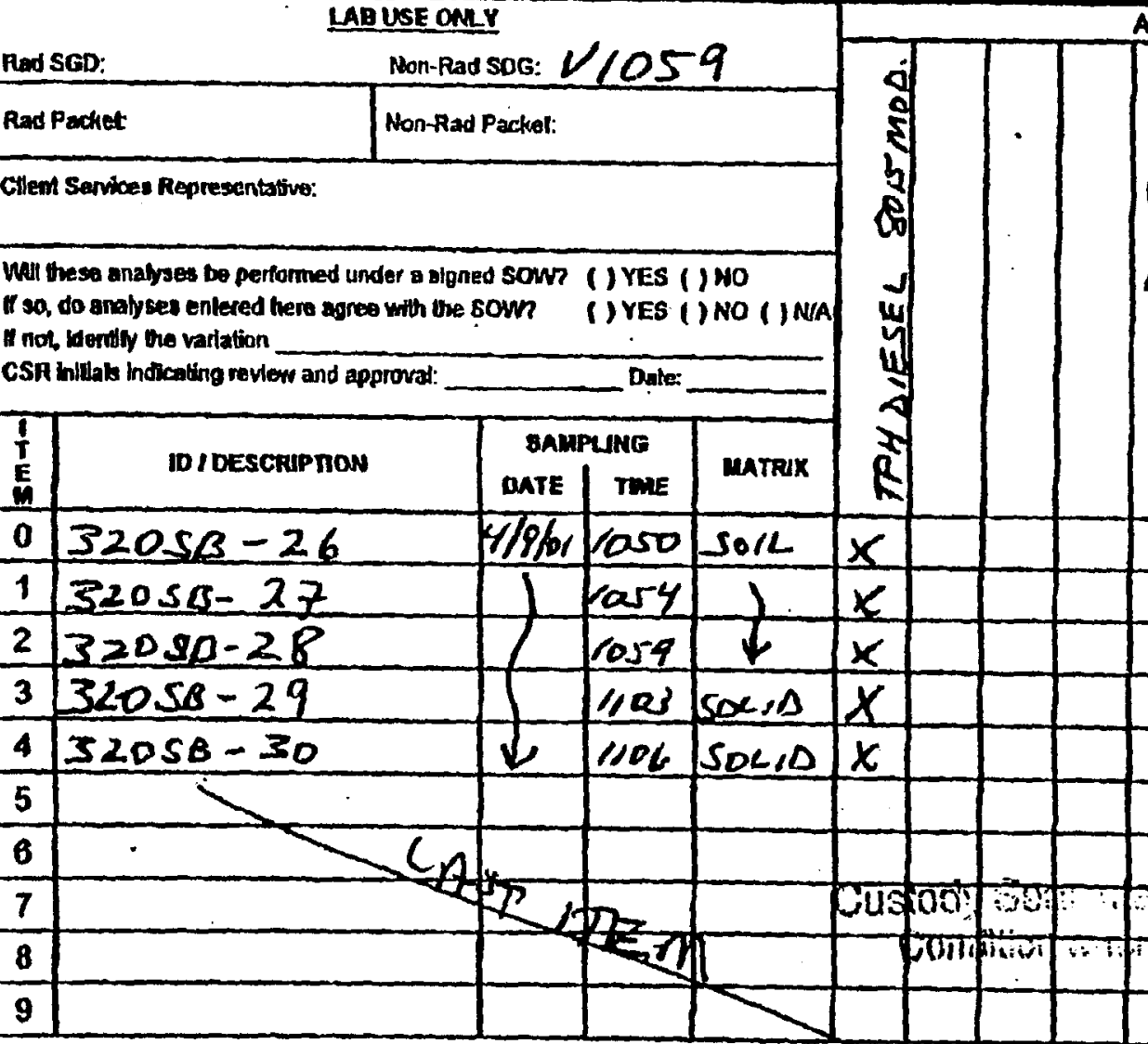

ANALYSES R METHOD

Tranater of samples submithed for analywes

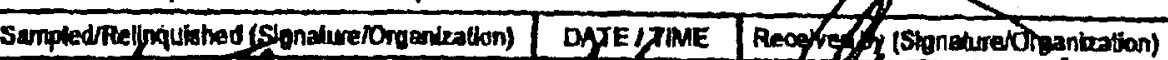

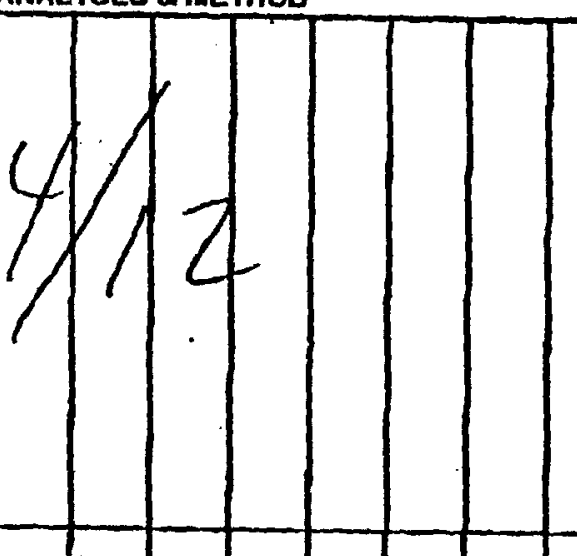
abmple materials.

\section{BALPLE RECEPT UAFORMANON}

Are all sample conlainers recelved htad I I Yes ( ) No Comments:

Do the labehe egree with this form? I Y Yos ( ) No

Comments:

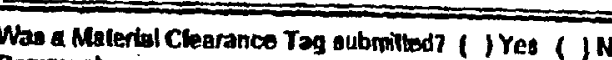

Commente:

ב

COMLENTS

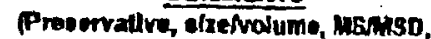

spoclal andyals, red matrix cods, count Umie, otc.)

$1-250 \mathrm{ml}$ glase j6c

per sample.

IEASPHALT IS NOZED N SAMPLE DO NOT

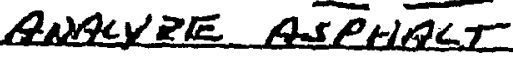

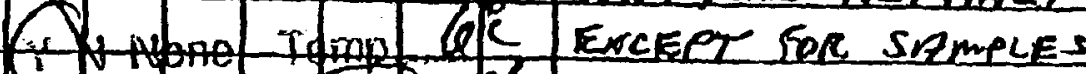

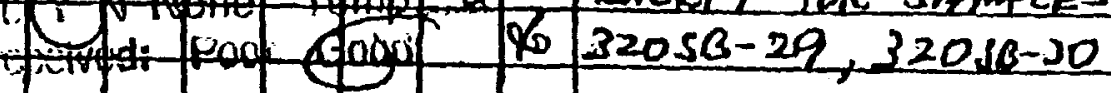

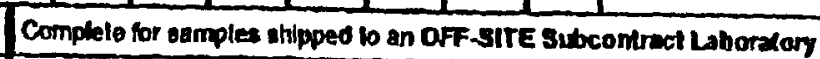

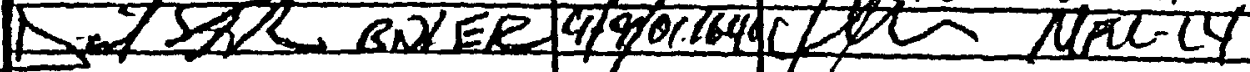

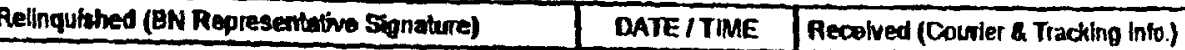

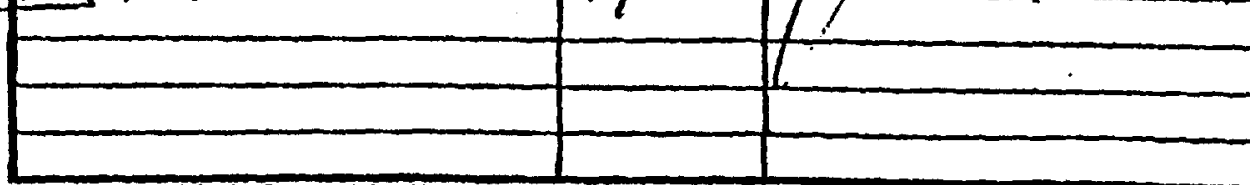

\begin{tabular}{|l|l|l|}
\hline Relinquished (Courter a Tracking into.). & DATE / TIME & Roosived (1si biel Sibconlractor Rep) \\
\hline
\end{tabular}

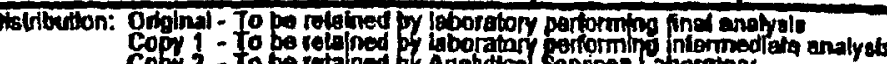
Copy 3 : To be retaned by sampled

\begin{tabular}{|c|c|c|}
\hline 0 & WAIE TIDAE & Rocoived (1si bei Sibconliactor Rep) \\
\hline Relinquitahed (Ist wer Suboontredtor Rep) & DATE/TMAE & Peocived (and ver Suboontraction Rep) \\
\hline
\end{tabular}




\section{TIER I \\ CHECKLIST}

This checklist is essentially a completeness review to ensure that all field and laboratory data and documentation are present and complete. The review is designed to be expeditiously conducted by designated, qualified BN ER personnel, upon receipt of the analytical data from the laboratory. During Tier I review, problems shall be identified, documented, and transferred with the data for a Tier II review. This review will be completed on all analytical data/laboratory results. Check for the following items:

'Chain of custody is complete and legible.

Cover letter (case narrative) reviewed for significant problems.

All requested analyses were performed on all samples.

Holding times are not beyond specified standards.

Note cooler temperature on cooler is not above 4 degrees Celsius.

Was the proper preservation and $\mathrm{pH}$ for each Matrix used?

Check the laboratory log-in report for completeness and errors.

All field forms are present and complete.

Report forms inventory includes all CLP or LP-like forms.

Reporting levels are at the appropriate level. 


\section{TIER I REVIEW}

\section{GENERAL INFORMATION}

1. Project Name or Sample Delivery Group (SDG): $1 / 1059$

2. Date Samples taken: $4 / \mathrm{c} / \mathrm{Cl}$

\begin{tabular}{|c|c|c|}
\hline SAMPLE NUMBER & DATE RECEIVED & DATE ANALYZED \\
\hline $320 \leq 5-16$ & $4 / 9 / 01$ & $4 / 11 / 01$ \\
\hline $32053-17$ & & $4 / 11 / 01$ \\
\hline $3<05-18$ & & $4 / 11 / 01$ \\
\hline $32056-17$ & & $4 / 11 / 01$ \\
\hline $32050-20$ & & $4 / 11 / 01$ \\
\hline $3<0,0-21$ & & $4 / 11 / 01$ \\
\hline $3205 B-22$ & & $4 / i / 61$ \\
\hline $32056-23$ & & $4 / 11 / 01$ \\
\hline 32050.24 & & $4 / 11 / 01$ \\
\hline $32056-25$ & & $4 / 11 / 61$ \\
\hline $32053-26$ & & $4 / 11 / 01$ \\
\hline $32056-27$ & & $4 / 12 / 01$ \\
\hline $32055-28$ & & $4 / 12 / 01$ \\
\hline $32056-29$ & & $4 / 12 / 01$ \\
\hline $32056-30$ & 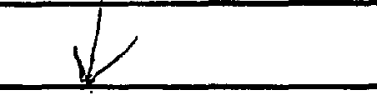 & $4 / 11 / 01$ \\
\hline & & \\
\hline & & \\
\hline & & \\
\hline & & \\
\hline & & \\
\hline
\end{tabular}


3. Date of Review: $4 / 12 / 01$

4. Chain of Custody (COC):

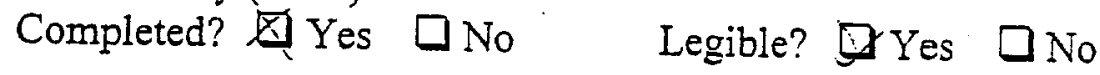

5. Is a cover letter/case narrative attached? ØY' $\square$ No

If 'yes,' has it been reviewed for significant problems?

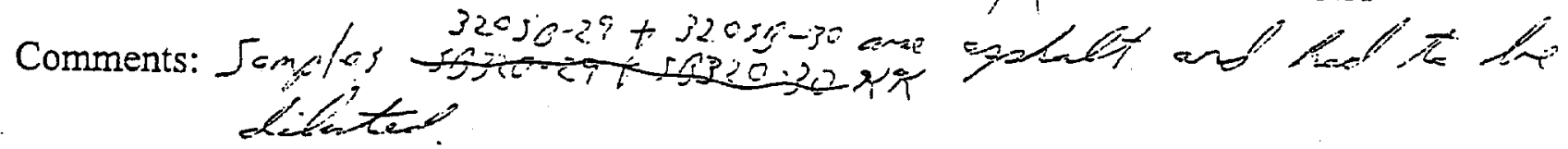

6. Analyses requested (Attach COC, Sample Request Form, and lab data packet to this review):

$\begin{array}{lll}\square \text { Total VOCs } & \square \text { Total BNA } \\ \square \text { TCLP VOCs } & \square \text { TCLP BNA } & \square \text { TCLP Metals } \dot{X}_{\text {TPH }} \\ \square \text { PCBs } & \square \text { Other: }\end{array}$

7. Were all requested analyses performed on all samples? Yyes $\square$ No

8. Temperature on cooler: $6{ }^{\circ} \mathrm{C}$ (parameters: $4^{\circ} \mathrm{C} \pm 2^{\circ}$ ) or $\square$ NA

10. Refer to Table 1. Was the proper preservation used? $\square$ Yes $\square$ No
If 'no,' then explain: 
TOTAL PETROLEUM HYDROCARBONS (TPH)

\begin{tabular}{|c|c|c|c|c|c|}
\hline PARAMETER & $\begin{array}{l}\text { EXTRACTION } \\
\text { HOLD TIME }\end{array}$ & $\begin{array}{l}\text { ANALYSIS } \\
\text { HOLD TIME }\end{array}$ & $\begin{array}{l}\text { DAYS } \\
\text { HELD }\end{array}$ & $\begin{array}{l}\text { PASS } \\
\text { Y/N }\end{array}$ & $\begin{array}{c}\text { SAMPLES } \\
\text { NOT PASSING } \\
\end{array}$ \\
\hline $\begin{array}{l}\text { Total TPH } \\
\text { EPA Method } \\
8015 \mathrm{M} \text { or } \\
8015 \mathrm{~B}\end{array}$ & $\begin{array}{l}\text { Liquids - } 14 \text { days } \\
\text { Soils - } 14 \text { days } \\
\text { Oil - } 14 \text { days }\end{array}$ & NA & 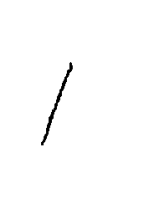 & & \\
\hline $\begin{array}{l}\text { Total TPH } \\
\text { EPA Method } \\
8015 \mathrm{M} \text { or } \\
8015 \mathrm{~B}\end{array}$ & NA & $\begin{array}{l}\text { Liquids - } 40 \\
\text { days } \\
\text { Soils - } 40 \text { days } \\
\text { Oil - } 40 \text { days }\end{array}$ & 1 & & \\
\hline \multicolumn{6}{|l|}{ Comments: } \\
\hline
\end{tabular}

Was TPH digestion done within the hold time limit?

Were analyses run within the hold time limit? 过Yes $\square$ No

A. TPH reported as: $7 \mathrm{mg} / \mathrm{Kg}$ or $\square$ ug/Kg Other:

B. Hits above detection level found in LB, RBS, FB, RB, or other QA samples? $\square$ Yes No If 'yes,' explain:

C. Did laboratory report indicate any problems? $\square$ Yes $\underset{\text { No }}{ }$ If 'yes,' explain: 


\section{SUMMARY}

Laboratory log-in report check for completeness and errors, Yes $\square$ No

Are all field forms are present and complete? Yes $\square$ No

Does the report forms inventory include all CLP or LP-like forms? $\square$ Yes $\square$ No

Are the reporting levels at the appropriate level? Yes $\square$ No

If 'no,' list the exceptions below:

\begin{tabular}{|l|l|l|l|l|}
\hline SAMPLE D & $\begin{array}{c}\text { PARAMETER } \\
\text { ANAIYSIS }\end{array}$ & $\begin{array}{c}\text { REPORTING } \\
\text { LEVELS }\end{array}$ & $\begin{array}{c}\text { ACTUAL } \\
\text { LEVEL }\end{array}$ & COMMENTS \\
\hline & & & & \\
\hline & & & & \\
\hline & & & & \\
\hline
\end{tabular}

Was the sample courit/type consistent with the COC? Yes $\square$ No

Were the results reported for both the field and laboratory QC samples? Yes $\square$ No

Is the analysis count/type consistent with the COC? Yes $\square$ No

Was the correct sample matrix used for each sample?

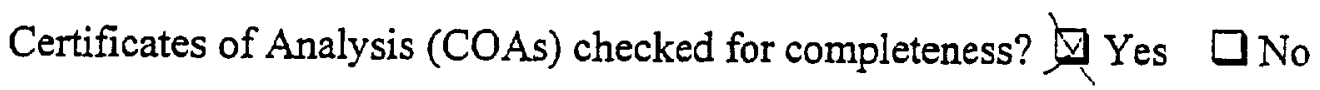

Condition-upon-receipt variance form included? $\square$ Yes $\square$ No 送N/A

Did the deliverable meet the overall objectives of the project?

16. Are all signatures in place? On COC2 9 Yes $\square$ No On Data pkg.? Explanation for any problems: 


\section{SUMMARY (cont.)}

18. Overall Comments:

19. Reviewed by:

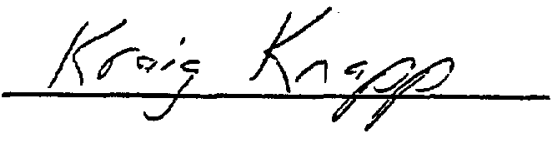

20. Task Manager or TPO
Date: Signature

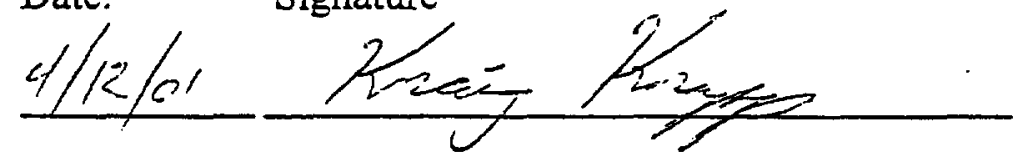

Date:

Signature 
CIIENT: : Bechtel Nerid

P.O. Box 98521, MS NIS273

ATIN:

Las Vegas, NV 89193-8521

Ted Redding

PROJECT NAME: VIO34

PROTECT NUMOBER: 23081

NEL ORDERDETOLOBY4

Armebed are the andytieal results far samples in support of tio aboverefereneed project

$$
20
$$

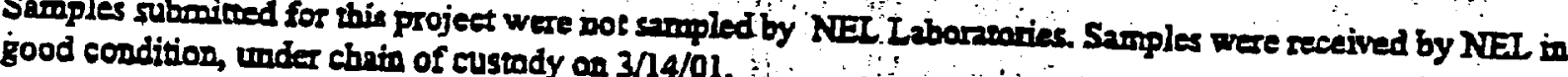

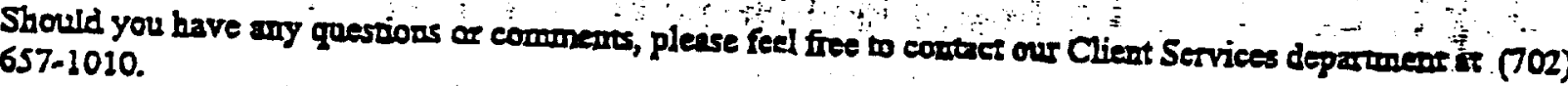
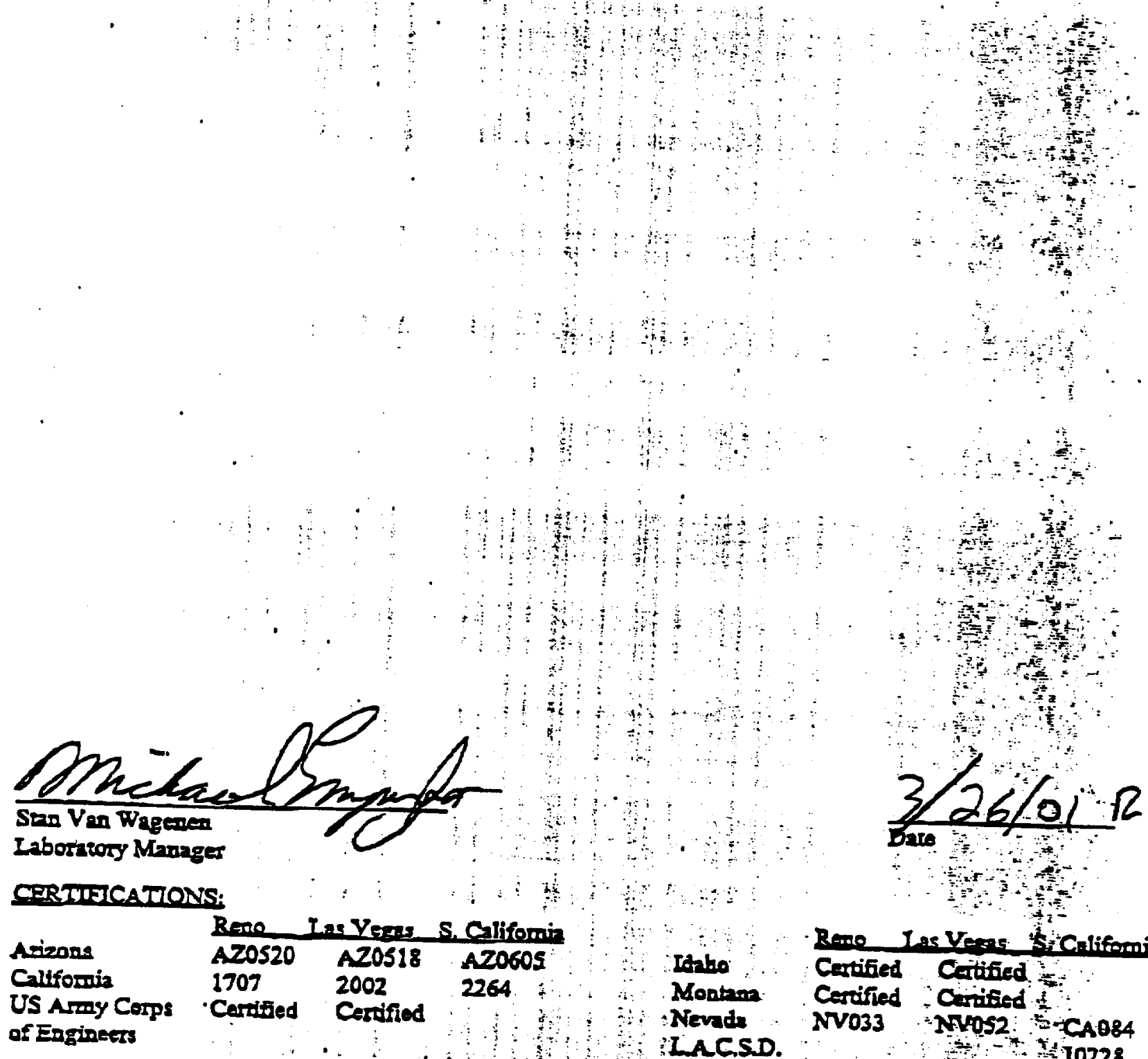

Into

Montan

Nevado

TACSD. 


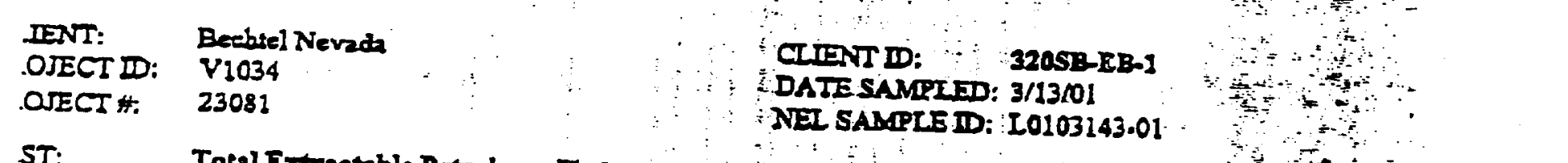

$\begin{array}{ll}\text { ST: } & \text { Total Extractoble Petrolem Hydrocarbond The Finger Frint by DA Method 8015M, July } 1992 \\ \text { IIHOD: } & \text { EPA 8015M }\end{array}$

ITRD: Aqueous

UTION: 1

ANAIYST: $\quad$ CCS - Las Vegar Divisiom

EXTRACIED: : 3/16/01

ANAIYZED: $\quad 3 / 26 / 01$

\section{RAMETER}

oline Range (C8-C12)

sel Range (C12-C22)

Range (C12-C34)

Result : Reporting

b

ALIYY CONTROL DATA:

rogete

scosane

- Not Derected

report shall not be reproduced cacest in full without the writhen uproval of the laboretory. 
IENT: Bechrel Nevada

OJECT ID: 'Y1034

OJECT \#: 23081

STi

ITHOD:

IRD:
Tozil Extracent

EPA 8015M

Aqueous
CIIENTD:

DATE SAMPLED: NA

NEI SAMPLE ID: 010316IPEW-FP-BIT

\section{RAMETER}

sline Range (C8-C12)

E! Range (C12-C2)

lange (C12-C34)

1

\section{ILTTY CONTROL DATA:}

-ogate

icosane

\section{- Nor Dereeted}

report shall not be repproduced exceps in fill, withovi the written opproval of the laboratory.

\section{ENI! Bechtel Nevade}

JECT ID: V1034

ECI 4 : 23081

I:
IOD: $\quad$ Pargeable I
EPA 8015M

RX. Solid
X Recorery

i: B4
CLIDNT ID: s. Mothod Blank

DATE SAMPLED: NA

NNEL SAMPLE ID: 0103200ASIBIK
CCS Method 8015ng Juby 1992

CCS - Las Vegas Division

ANAIYST:

EXIRACIED: $\quad 3 / 16 / 01$

ANAI YZED: 3/16/01 and

Repoting

Ethitt

$05 \cdot \operatorname{ming} 2$

$0.5 \operatorname{mg} \pi$

0.5 men

. 05 mer

incong

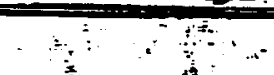

Aceeptabie Range

$60-121$

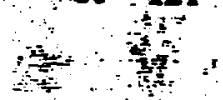

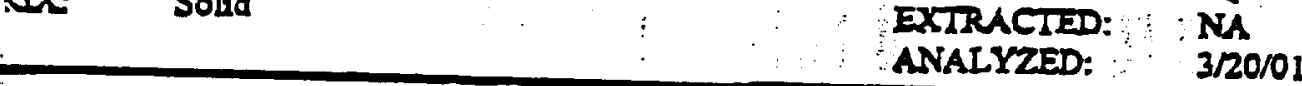

\section{WMETER}

ine

"ITY CONTROL DATA:

zate

Trifluosotobuene

ERecovery

108
JQT - Las Vegas Division

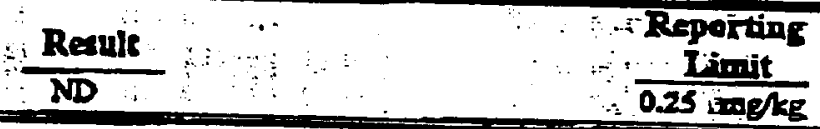

Not Detected

port shall not be reproduced acept in fill, without the writen approval of the laborazory. 





CLIENT:

Bechuel Nevada

P.O. Box 98521, M/S NTS273

AIDN:

Las Vegas, NV $89193-8521$

PROJECT NAME: V1034

PROIECT NUNRER: 23081

NEL ORDER ID: L0103143

Attached ate the analytical ressils for sarmples in sipport of the above refereneed project.

- Samples submined for this project were not sampled by NEI Labentosies. Samples were received bx NEI in Should you bave any questions or comments, please feel free ro connet our Client Services doparment at (702)
$657-1010$.

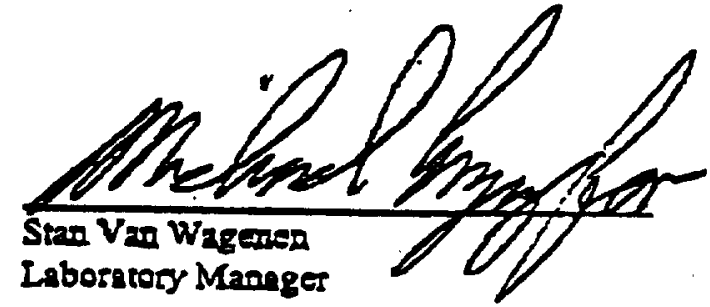

\section{CERTIFICATIONS:}

\begin{tabular}{|c|c|c|c|}
\hline & Reno & Ins Veges & S. Clifomis \\
\hline $\begin{array}{l}\text { Arizona } \\
\text { Californiz } \\
\text { US Army Corpa }\end{array}$ & $\begin{array}{l}\text { Az0520 } \\
1707 \\
\text { Cerified }\end{array}$ & $\begin{array}{l}\text { A70518 } \\
2002 \\
\text { Cerdified }\end{array}$ & $\begin{array}{l}\text { A20605 } \\
2264\end{array}$ \\
\hline
\end{tabular}




\begin{tabular}{|c|c|c|}
\hline $\begin{array}{l}\text { LIENT: } \\
\text { ROJECI D; } \\
\text { ROJECT \#: }\end{array}$ & $\begin{array}{l}\text { Bechtel Nevada } \\
\text { V1034 } \\
23081\end{array}$ & $\begin{array}{ll}\text { CLIENT ID: } & 3205 B-1 \\
\text { DATE SAMPLED: } & 3113 / 01 \\
\text { NEI SAMPLE DD: I0103143-02 }\end{array}$ \\
\hline
\end{tabular}

EST:

ETHOD:

ATRX:

IIUTION:
Purgeable Total Petroleum Bydrocarbons by Mod8015-GRO ERA 8015M Solid 1
ANALYST: EXTRACIED: ANALYZED:
JQI - Las Vegas Division

NA 3/20/01

$\frac{\text { Result }}{\text { ND }}$
Recovery

70
Reparting

Ifinit

$\therefore$ 0.25 singkg

IALTY CONTROL DATA:

irrogate

2-Trifluototoluere

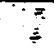

Acceptable Ranse

\section{- Not Detected}

i report shell not be reproduced exeept in full, without the written approval of the laborarory. 
IIPNI: Bechiel Nevada

'ROJECT ID: V1034

'ROJECT *: 23081

CLIENT D: 320SB-2 MSMSD

DATE SAMPLED: 3/13/01

EST:

IETHOD:

Purgeable Total Petroleum Hydrocarbon by Mods015-GRO

IATRD:

EPA 8015M

IIIUTION: Solid

ANAIXST:

EXTRACTED: ANALYZED:

JQT - Ias Vegas Divisioń 1 $3 / 20 / 01$

\section{ARAMETER}

scoline

Recult

UALTT CONTROL DATA:

urrogate

a,a-Trifluorowluere

\% Recovery

79

D - Not Detected

its report shall not be reproducad exeqt in full, withoul the written approval of the laboratory.

Soporting

$\frac{\text { Limit: }}{0.25 \text { ing/kg }}$

$\therefore \therefore$

Accépafile Range

$65 \div 120$

$\therefore$.

- 2

$\because$

$\therefore$

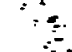

$\because$

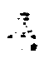




$\begin{array}{lll}\text { LIENT: } & \text { Besbel Nievads } & \text { CIIENT D: } \\ \text { ROJECT DD: } & \text { V1034 } & \text { DATE SAMPLED: 3/13/01 } \\ \text { ROJECT } & 23081 & \text { NEI SAMPLED: L0103143-04 }\end{array}$

$\begin{array}{ll}\text { EST: } & \text { Purgeable Total Petroleum Hydrocarbons by ModB015-GRO } \\ \text { ETHOD: } & \text { EPA 8015M }\end{array}$

ATRIX: Solid

IUTION: 1

ANAIYST: JQT - Las Vegas Division

EXTRACTED: NA

RAMTETER

soline

IALITY CONTROL DATA:

rrogate

a-Trifluororohuene ANAIYZED: $\quad 3 / 20 / 0$

- Nor Decected

isport shall not be neproriced exegp in full, without the wrtuen approval of the laboratony.

Recovery

79

Result

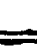

y

rovel of the laboratory.

\section{Reporting \\ Ilinte \\ $0.25 \mathrm{i}=2 \mathrm{~g} / \mathrm{kg}$}

...

Acceptible Range

$65-120$

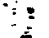

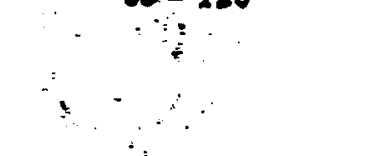




$\begin{array}{ll}\text { IIENT: } & \text { Bechel Nevada } \\ \text { ROJECT ID: } & \text { V1034 } \\ \text { ROJECT H: } & 23081\end{array}$

CLIENT D: $\quad 3205 B-4$

DATE SAMPLED: $3 / 13 / 01$

ROJECI

NEL SAMPLE D; L0103143-05

EST: Purgeable Toral Petroleum Fydrocarbons by Mod8015-GRO IEIHOD: EPA 8015M LATREX: Solid IIUTION: 1

ANALYST:

EXIRACTED: ANALYZED:
JQT - Las Vegas Division:

NA 3/20/01
AFAMETER

Resule

ssoline

ND

\section{$\%$ Recovery}

67

J - Nol Detected

is report shall not be reproduced exeept in full, without the written opproval of the laboratory.

Reporting.

Itonit

0.25 mg/kg

Irrogate

3,2-Tritluorosoluene

Acceperable Ragge

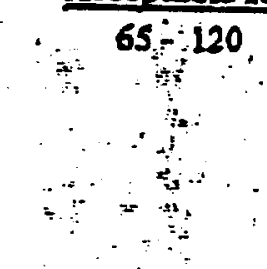




\begin{tabular}{|c|c|c|}
\hline $\begin{array}{l}\text { ILENT: } \\
\text { ROJECI D: } \\
\text { ROJECI \#: }\end{array}$ & $\begin{array}{l}\text { Bechiel Nevada } \\
\text { V1034 } \\
23081\end{array}$ & $\begin{array}{ll}\text { CLIENT D: } & \text { 32058-5 } \\
: \text { DATE SAMPLED: 3/13/01 } & \text { NEL SAMPLE TD: L0103143-06 }\end{array}$ \\
\hline
\end{tabular}

ESI: $\quad$ Purgeable Total Petroleum Hydrocarbons by Mod8015-GRO ETHOD: EPA 8015M ATRE: Solid IIUTION: I

ANALYST:

EXTRACIED: ANALYZED:

\section{IRAMETER}

130line

UALITY CONTROL DATA:

irrogate

ba-Trifluorololuene

\% Recavery

65

i- Not Deteced

is report shall riot be reproduced except in full, withous the written approval of the laboracony.
JQT - Las Vegas:Dirisios

NA 3/20/01

Result

$\therefore$ Itinlt

$-0,25^{\prime} \mathrm{mg} / \mathrm{kg}$

\section{Acceptoble Range}

$65 \div 120$

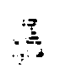


IENT: Bechrel Nevada

ROJECT ID: V1034

ROJECI H: 23081

CLIENT D: $\quad 3205 B-6$

DATE SAMPLED: $3 / 13 / 01$

NEL SAMPLE ID: L0103143-07

EST: $\quad$ Purgeable Total Petroleum Hydrocarbons by Mod8015-GRO

IITIOD: EPA 8015M

CATRL: Solid

ANAIYST!

IIUTION: 1

EXIRACIED: ANALYZED:

JQT - Las Veges. Divirion: :

NA

$3 / 20 / 01$

\section{ARAMETER \\ ssoline}

Reourt

ND

\% Recover

irrogate

2,2-Triguororobreno

72

2. Nor Detected

is repor shall not be reproduced except in full, without the writen approvel of the laboratory.

Acceptible Range $65-120$

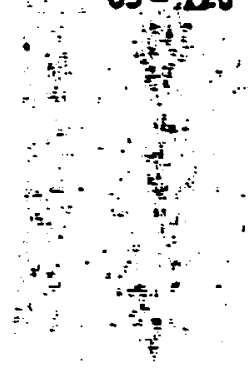




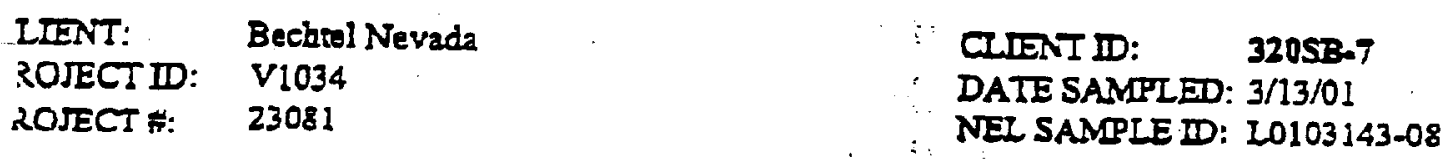

5ST: Purgenble Tosal Perroleum Eydrocarbous by Mods015-GRO

ETHOD: EPA 8015M

AIRL: Solid

IIUTION: ?

ANAIYST:

EXIRACTED: ANAIYZED:
JQT - Las Vegas Division

NA. 3/20/01

\section{RAMETER}

soline 1

$\frac{\text { Reralt }}{\text { ND }}$

\% Recovery

Irrogate

2-Trifluorotoluene

77

\section{- Nor Detected}

- report shell not be reproduced ercept in full, without the wrinen approval of the laboretory: 
$\begin{array}{ll}\text { LIENT: } & \text { Bechell Nerada } \\ \text { ROJECT DD: } & \text { V } 1034 \\ \text { ROJECT \#: } & 23081\end{array}$

CLIENT DD: $\quad 320 S B-8$

DATE SAMPLED: 3/13/01

NEL SAMPLE DD: L0103143-09

$\begin{array}{ll}\text { EST: } & \text { Purgesble Total Petroleum Fydracarbons by Mod8015-GRO } \\ \text { IETHOD: } & \text { EPA 8015M }\end{array}$

LATRIX: Solid

IIUTION: 1
ANAIYST:

EXIRACIED;

ANAIYZED:
JQT - Las Vegas Bivisioñ

NA

320/01

$\frac{\text { Recult }}{\text { ND }}$

$\%$ Recovers

69

J - Nor Deteeted

is report shall not be reproduced exept in fill, without she written opproval of the leboratory. inrogate

4i-Trifluorotolueac

$$
6
$$

proval of the leboratory.


LIENT: Beeitel Novada

ROJECT ID: V1034

ROJECT \#; 23081
CLIENT ID:

DATE SAMPLED: 3/13/01

NEL SANPLE ID: I0103143-10
EST: Purgesble Total Petrolenm Hydrocarbous by Mod8015-GRO

ETHOD: EPA 8015M

ATRDX: Solid

IUTION: 1
ANAIYST:

EXTRACTED: ANAIYZED:
JQT - Les Vegas Divisió

NA 3/20/01

\section{ARAMETER \\ soline \\ IALITY CONTROL DATA:}

irrogete

,a-Irifluorotoluene

$\%$ Recorery

74

1- Not Deteeted

is report shall not be reproduced acept in full, without the written approval of the laborasory: 
ZLIENT:

'ROJECT ID

'ROJECT \#:

.EST:

IETHOD;

SAIRX:

IIIUTION:
Becbee] Nevadz

V1034

23081
CLIENT ID: $\quad 320-5 B-10$

DATE SAMPLED: 3/13/01

NEI SAMPLE DD: L0103143-11

Purgaeable Total Petroleom Hydrocarbous by Mod801S-GRO EPA $8015 \mathrm{M}$ Solid 1

ANAIYST:

EXIRACIED: ANALYZED;

JQT - Las Vegas Division

NA 3/20/01

\section{ARAMETER}

asoline

UALITY CONTROL DATA:

urrogate

3,2-Trituosotohene

\section{Reult}

ND

\section{$\%$ Recovery}

78

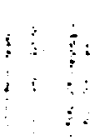

D - Not Detected

his roport shall nos be reproduced except in full, wichout the wrizen approvel of the laboratory.

$\frac{\ldots \frac{\text { Repporting }}{\frac{\pi \text { Limit }}{025=\operatorname{mg} / \mathrm{gg}}}}{\frac{\text { Acceptrble Rarge }}{65 \cdot 120}}$




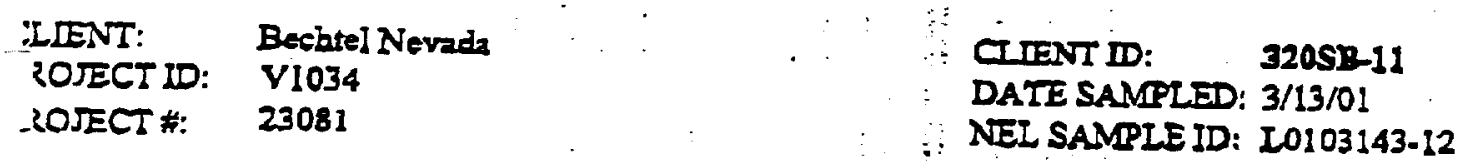

CIIENT ID: 320SR-11

DATE SAMPLED: 3/13/01

NEI SAMPLE ID: L0103143-12

RAMETER

ALITY CONTROL DATA:

urtogate

As-Trifluoropluene

I - Not Detected

i report shall not be reproduced exept in full, withous the witton approval of the laboratory.
$\%$ Recovery

96
Reaul

$\therefore$
ANAIYST:

EXTRACTED: ANALYZED:

JQT - Las Vegas Division

NA $3 / 20 / 01$

\begin{tabular}{c} 
Repording \\
0.25 mogng \\
\hline
\end{tabular}

\begin{tabular}{c} 
Repording \\
0.25 mogng \\
\hline
\end{tabular}

\begin{tabular}{c} 
Repordag \\
0.25 megke \\
\hline
\end{tabular}

Acoeptible Reage $65+120$

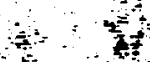


ZLIENT:

'ROJECT D:

'ROJECT \#:

EST:

IETHOD:

LATRIX:
Besbtel Nevada

V1034

23081
CITENT D:

DATE SAMPLED: 3/13/01

NEL SAMPLE D: L0103143-13

Pargeable Total Petroleum Hydrocarbons by Mod8015-GRO .. EPA 8015M

IIIUTION:
Solid

1
ANALYST:

EXIRACTED: ANAIYZED:
JQT - Las Vegas Division

NA 3/20/01

ARAMETER

asoline

UALITY CONTROL DATA:

urrogate

3,a-Trithorotoluese

D - Not Detected

is report shall nat be neproduced exaept in full, withour the whiten approvel of the laboretory. $\therefore$ Rearit $\therefore$ Retarting

ND

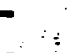

\%ecovery

85 Ininit $\therefore \quad .65 \leqslant 120$
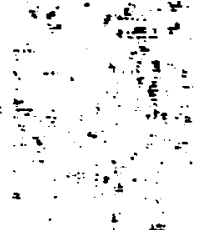

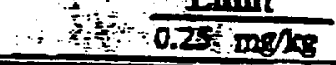

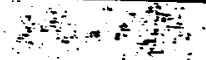

Ficcóptable Range
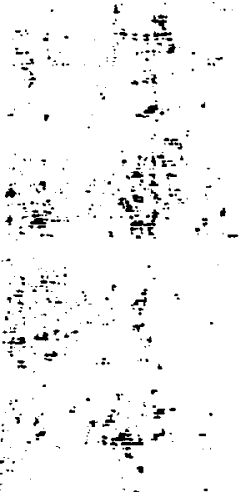


\begin{tabular}{|c|c|c|}
\hline $\begin{array}{l}\text { IIENT: } \\
\text { ROJECT D: } \\
\text { ROIECT \#: }\end{array}$ & $\begin{array}{l}\text { Beehred Nevadz } \\
\text { V1034 } \\
23081\end{array}$ & 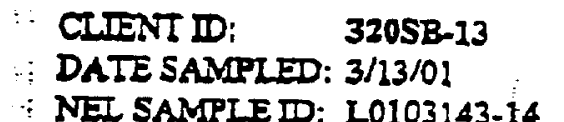 \\
\hline
\end{tabular}

-EST: Purgesbie Tocal Petraleum Hydrocerbons by Mod8015-GRo IETHOD: EPA 801SM SATRD: Solid IIITION: 1

i: ANAIYST:

\section{ARAMETER}

asoline

TAWTY CONIROL DATA:

rrogate

,a-Irifluorotoluene

\section{\% Recovery}

\section{1- Noi Detected}

is report shall not be reproduced except in full, whthour the writaen approval of the laboratory.
EXIRACTED: ANALYZED:

71

JQT - I as Vegas Divisiod

$\mathrm{NA}$ 3/20/01

Berult " $"=$ Repordog

ND

10.23 . IE $/ \mathrm{kg}$

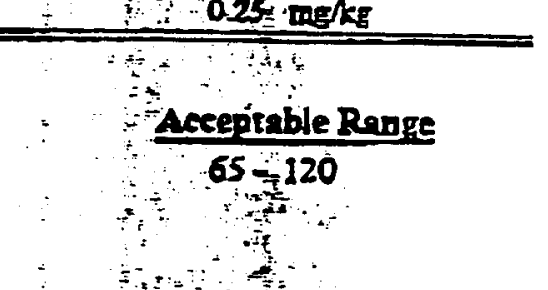




$\begin{array}{ll}\text { ITENT: } & \text { Becatel Nevada } \\ \text { ROJECT ID: } & \text { V1034 } \\ \text { ROJECT \#: } & 23081\end{array}$

$\because$ CIIENT D:

3205B-14

DATE SAMPLED: 3/13/01

NEI SAMPLE ID: L0103143.15

$\begin{array}{ll}\text { EST: } & \text { Purgeable Total Petroletim Hydrocarbons by Mod8015iGRO } \\ \text { IETHOD: } & \text { EPA 8015M }\end{array}$

IATRD: Solid

IIUTION: -I

ANAIYST:

EXTRACTED:

JQT - Ias Vegas Division :

ANAIYZZD:

NA

$3 / 20 / 0$

$\frac{\text { ARAMELER }}{\text { asoline }}$

Irrogate

ä, 2-Trifluorocoluene

Result

ND

$\therefore$ Treparting

$0.25 \mathrm{mg} / \mathrm{kB}$

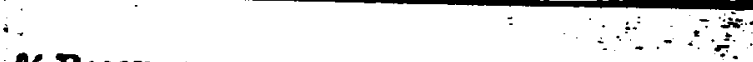

$\frac{92 \text { covery }}{97}$

D- Xot Deteetred

is report shall nor be reproduced except in full. wichous che writlen approvel of the laboretory.

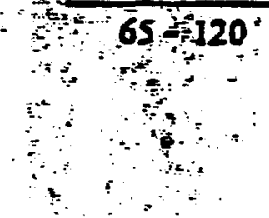$$
\text { ath }
$$ 


$\begin{array}{ll}\text { IIENT: } & \text { Bechwil Nevada } \\ \text { ROJECT ID: } & \text { V1034 } \\ \text { ROTECT \#: } & 23081\end{array}$

CLIENT ID: 3205B-15

DATE SAMPLED: $3 / 13 / 01$
NEI SAMPLE D: $20103143-16$

P.17/237

\section{ST:}

ETEOD:

IATRE:

Purgeable Total Petroleum Eydrocarbou by Modso15-Gro

Solid

EPA BOISM

IIUTION: 1

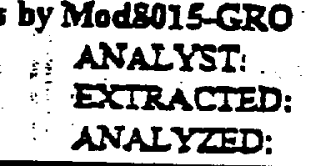

JQT - Les Vegus Divirion

FAMETER

asoline. 1

NA $3 / 20 / 01$

\section{ALITY CONTROL DATA:}

arrogate

a-Irifluorotoherese

Result . $\quad \vdots \quad-\quad$ Repordng

itinit

\section{- Not Deteeted}

..- report shall noz be reproctuced except in full, wichous the writen approvel of the laboritory. 


$\begin{array}{ll}\text { IIENT: } & \text { Bechrel Nevida } \\ \text { ROJECI ID: } & \text { V1034 } \\ \text { ROJECT \#: } & 23081 .\end{array}$

CItant D: $\therefore$ Method Blank DATE SAMPLED: NA

\section{EST:}

IEIHOD:

LAIRDX:

Purgeable Total Petroleum Eydrocarbons by Mod8015-GRo

NEI SAMPLE ID: O10320GAS-BLK

Method Blank

ANALYST:

EXIRACTED: ANAI YZED:
JQT - Las Vegas Division.

NA $3 / 20 / 01$

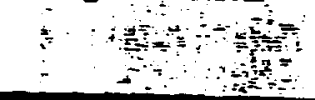

\section{ARANTEIER}

\section{3soline}

UALITI CONTROL DATA:

urrogate

3,3-Irifterotoluene

Solja

Reril

-ND

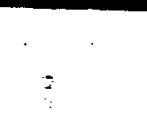

Neporting

$=0$ etini 0.25 ing $/ \mathrm{kg}$

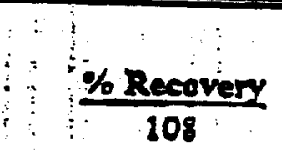

5- Not Derected

is report shall not be reproduced excopt in full, without the written opproval of ihe labaratory. 


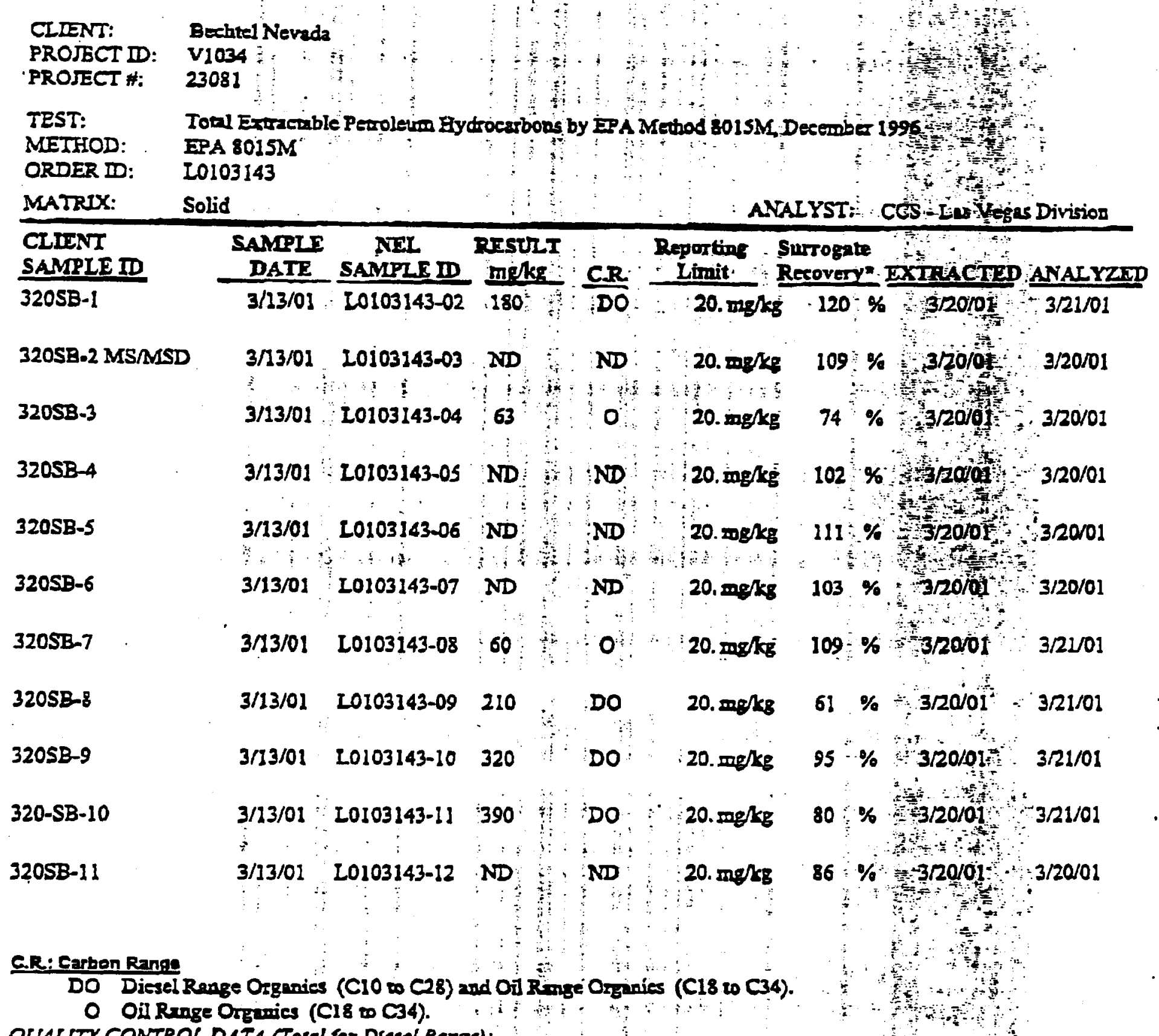

QUALITY CONTROL DATA (ToLal for Dresel Range):

Sample ID

Blenk, 010316TP -BLR

LCS, 010316TPHW-LCS

LCSD, 01031 GTPHW-LCSD

Blank, 010320TP -BLK

LCS, 010320IPHS-LCS

LCSD, 010320TPESICSD

MS, 010320TRHS-MS

MSD, 010320 TPHS-MSD

Resule Acciptable Ronge Surrogate Recovery* Sample Nombe

- Surrogate used was Octacosane, acceptance limito 65-130\%.

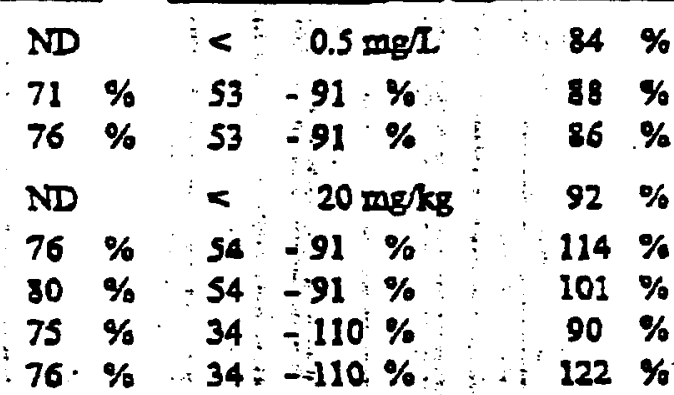

ND - Not Detected

This report shall not be reproduced except in sull, whoug the written approval of the taboratory:

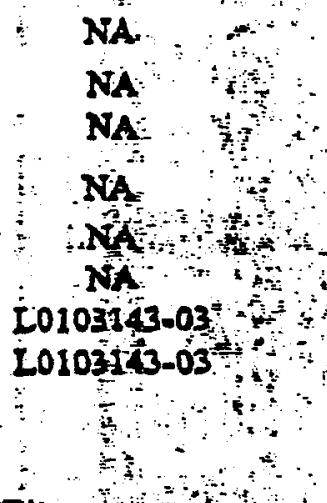

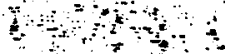

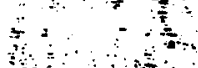




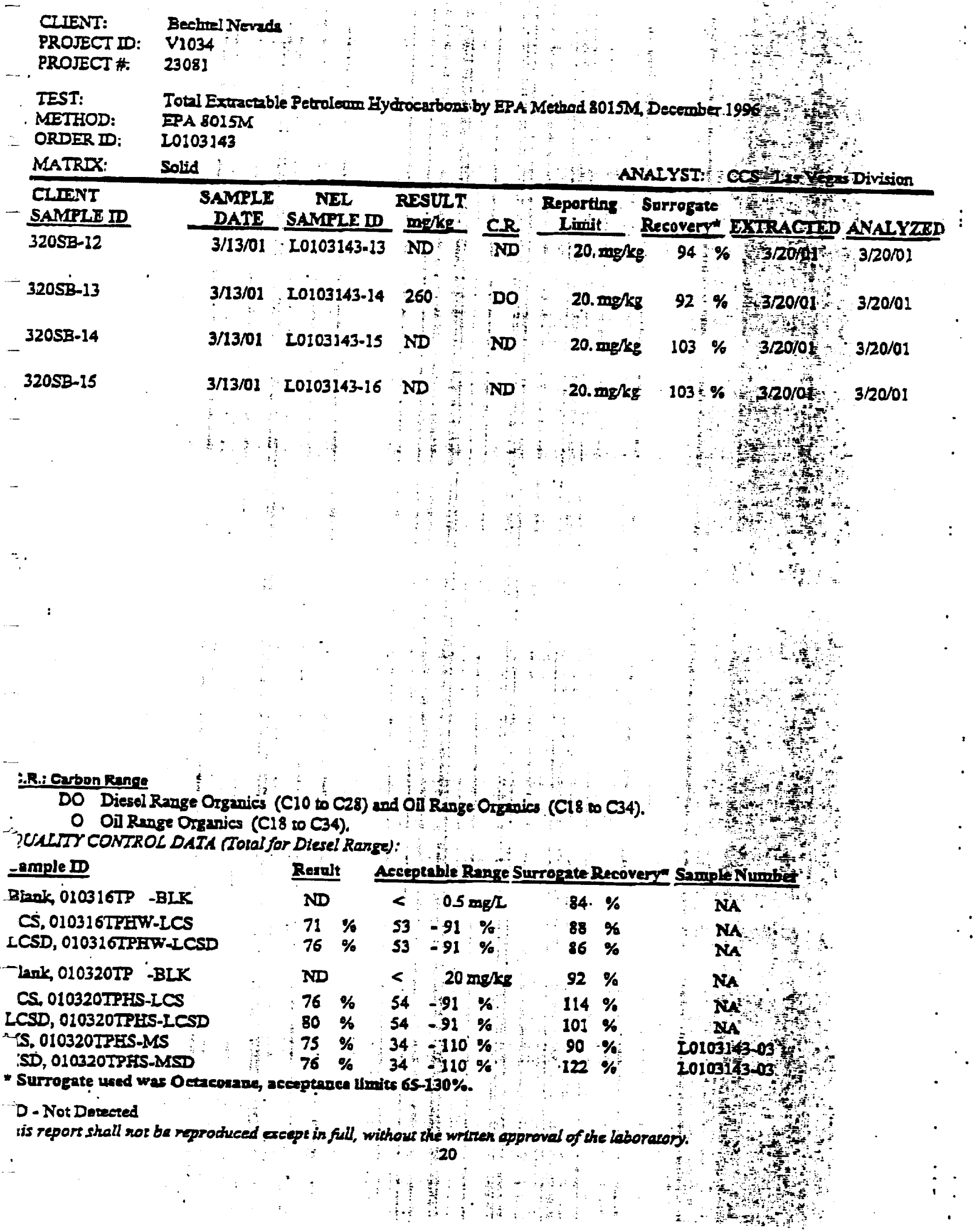







culuirs.

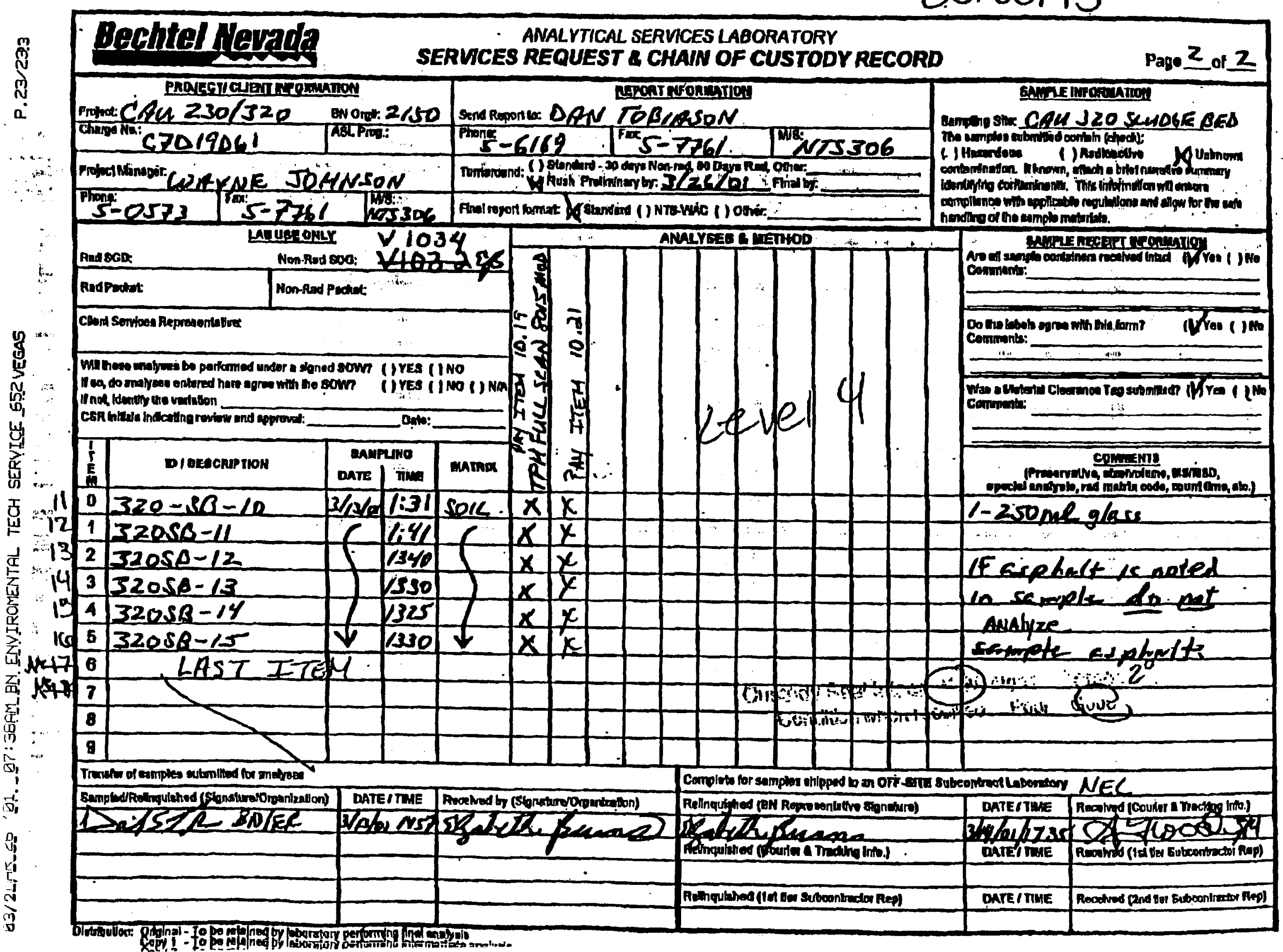




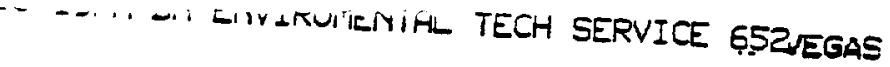

Data FIIE : C:IHECHEMIZIDATALitarion Repozt (OT Reviewed)

Acq on : 16 MaI 20101 111.13316. SECIO3I6-29.D

Misc : I0103143-01 $11: 13 \mathrm{pm}$

IntFile: WATER SAMPLE

Quant mime: events.e

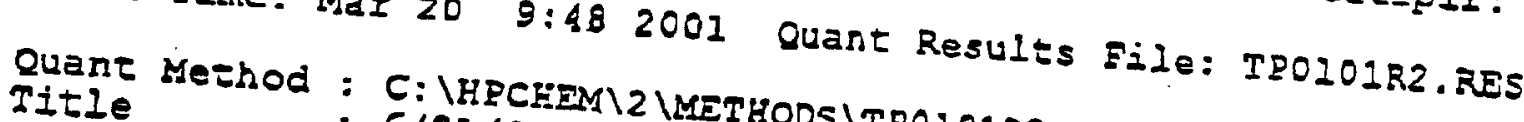

: 6/21/99 Seymoli EIOAt THH
Rast Update : Wed Jan 10 15:10:47 2001

DataAcq Meth : TP9907Rl.M

Volume Inj.

Sigral Phase:

Signal Info:

Compound

$$
\begin{aligned}
& \text { Viai: } 26 \\
& \text { Opezator: CCs } \\
& \text { Inst : 5eymour } \\
& \text { Multiplr: } 1.00
\end{aligned}
$$

$M$ Chemstation

Integracor)

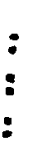

:

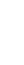

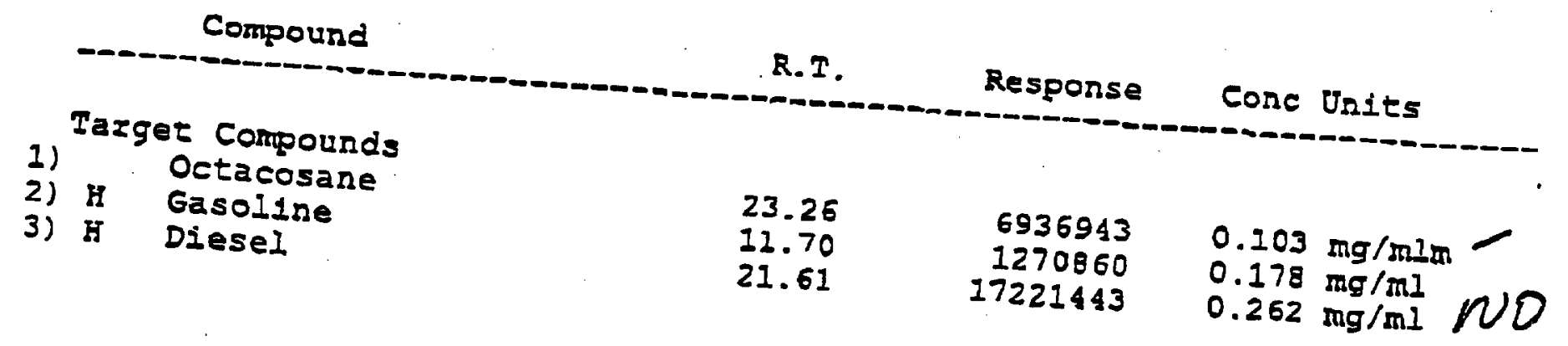




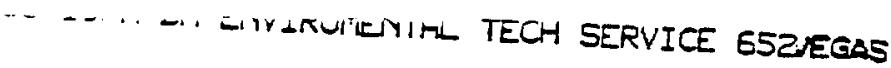

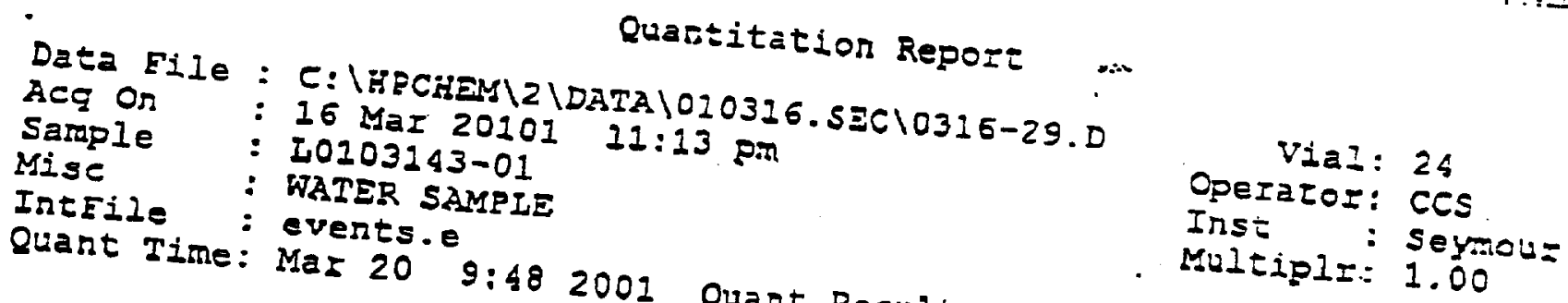

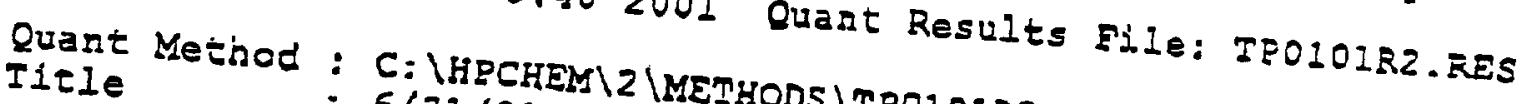

: 6/21/99 STIZLMETHODSITPO101R2.M (ChenStation Integst

Iast Opdate : Wed Jan Seymour Eront TPH

Response via : Multiple Jeve 15:10:47 2001

Dataxcq Meth: TPltiple Level Calibration

Volume Inj.

Sigral Phase:

Signal Info:
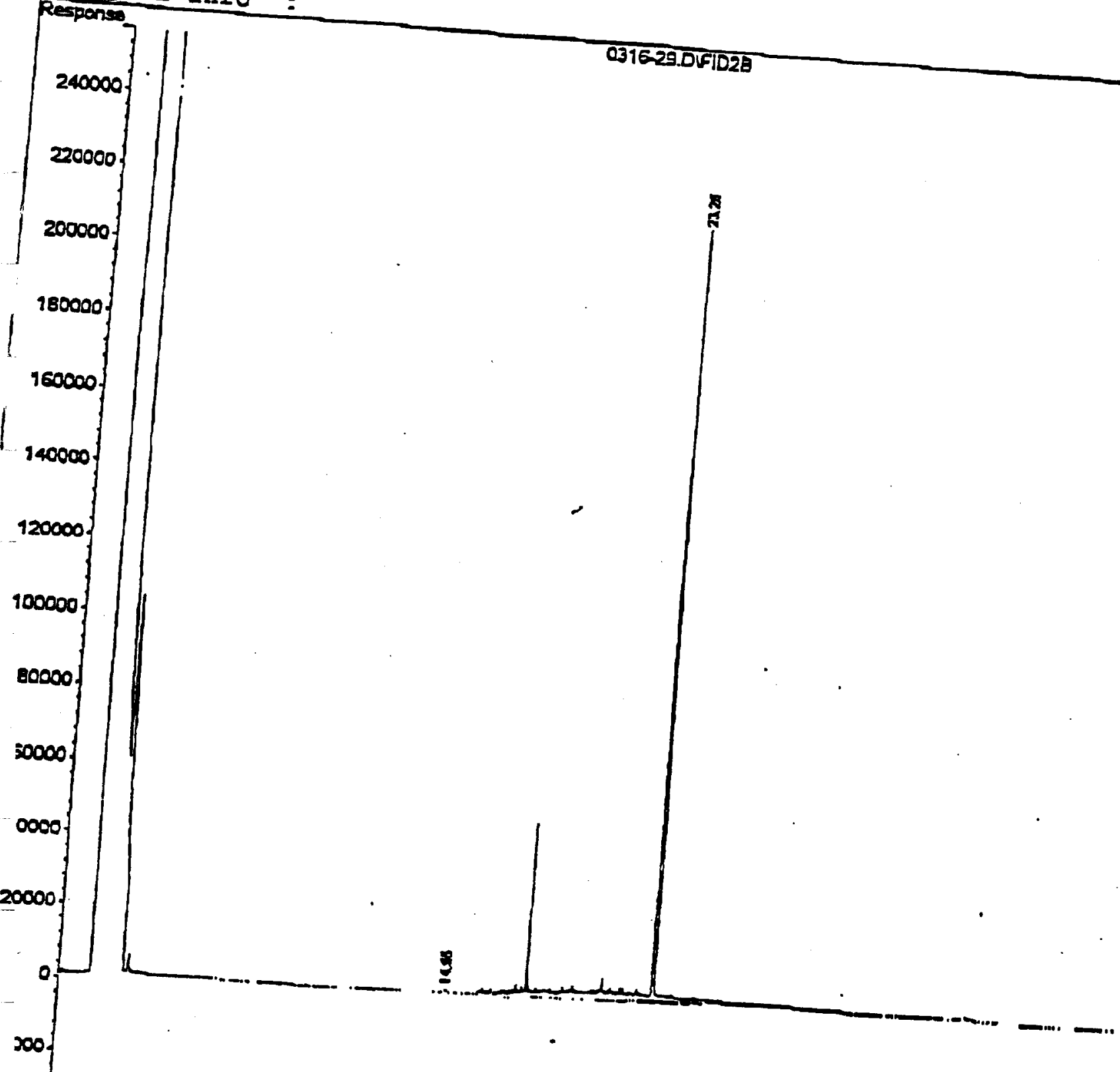

031620.DFID2B

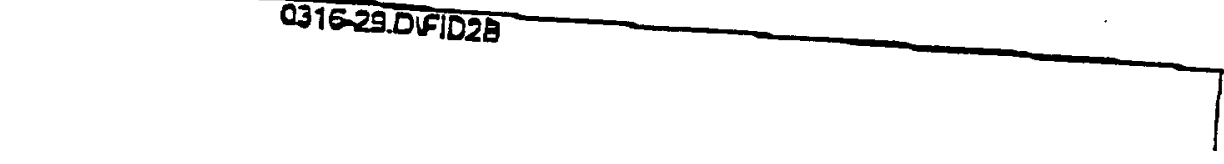

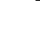

(1)

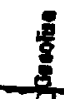

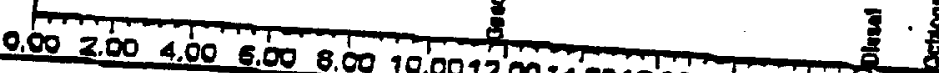

$22 . D$ TPOIOIR2.M

Iue MaI $20 \quad 09: 52: 52$ 200I 


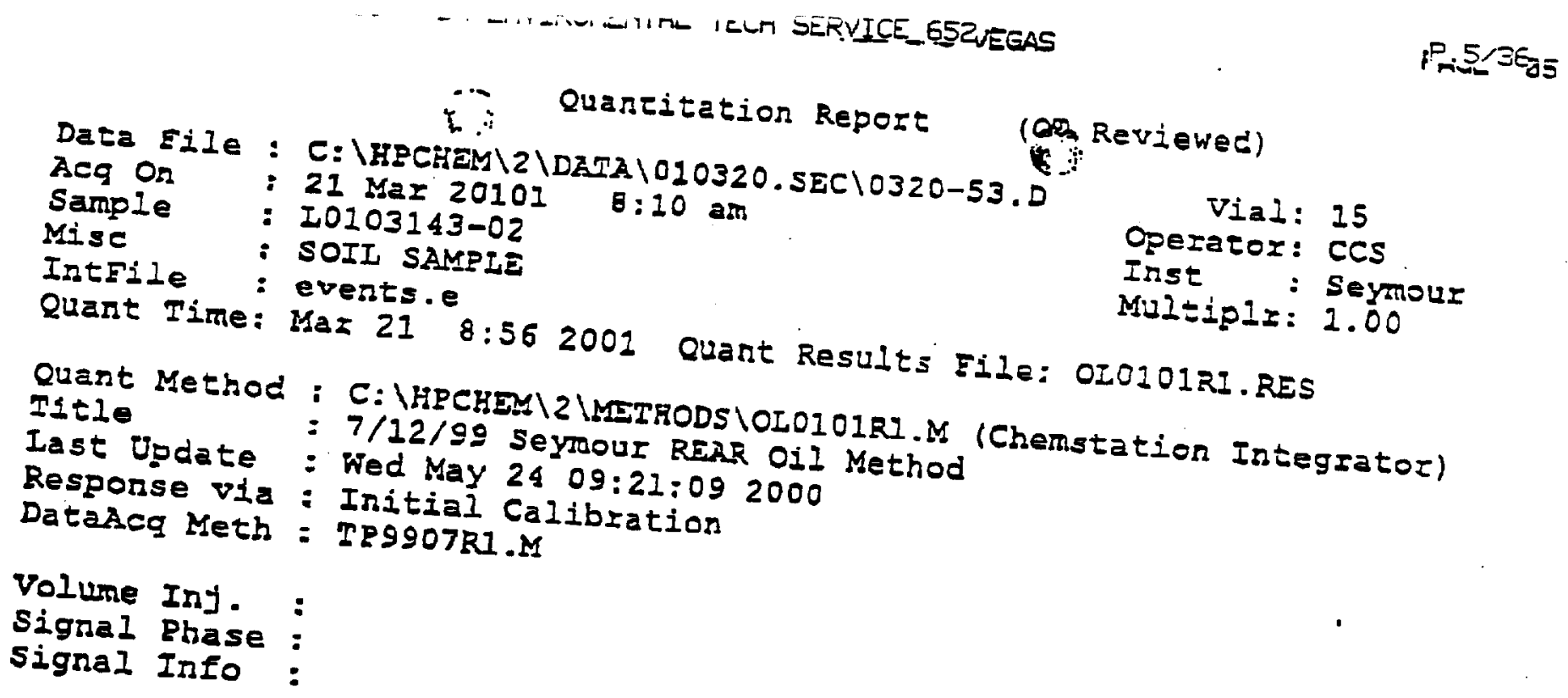

Pr is

Data File : c: friction Quantitation Report (OQ Reviewed)

Aeq Of : 21 Mar 2010I

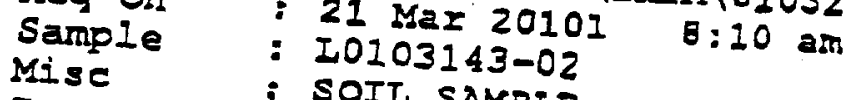

IntFile: SOIL SAMPLE

Quant rime: events.e

Vial: 15

Operator: CCS

Inst : Seymour

2001 Quant Results El le: OI0101RI.RES

TItle : $7 / 12 / 92$ Seymour RSIOLOI01RI.M (Chemstation Integrator)

Response via: Wed May 24 09:21:09 2000

Dataleq Meth : Initial Calibration

Volume Ing. :

Signal Phase:

Signal Info :

Compound

R.T. Response Conc Units

$$
\text { Target Compounds }
$$

.) H Oil

28.53

378982143

$$
\begin{array}{r}
5.362 \\
0.120 \\
\hline 5.242
\end{array}
$$

5. $242 \times 1.0$

$30.01 \times 1000=175=180$

T. Delta > 1/2 Windily

53.D OLO101R1.M

Wed Mar 21 08:56:25 2009

(m) =anus ins. 
Data File = cilHpcian Ruantitation Report

Aeg on

Sample

Misc

IntFile

: Ci 21 MPCEEM 20101 DATA 1010320. SEC $10320-53.0$

: I0103143-02

8:10 am

: SOIL SAMPLE

Quant Tine: events.e

Quant Tine: Mar 21

Quant Method : C: IHPCHEMI2lMETHODSIOI
Title

Last Update

Last Update : Wed May 24 09:21:09 2000
Response via: Multiple Ievel Calibrat
DataAcg Meth: TRgold

: 7/12/99 SE LMETHODS IOLO101RI.M Chemstation

Wed 9 Seymour REAR Oil Method

- Multipl 24 09:21:09 2000

Volume inj: TR9907RI.M

Volume Inj: :

Sigral Phase:

Sigral Info:
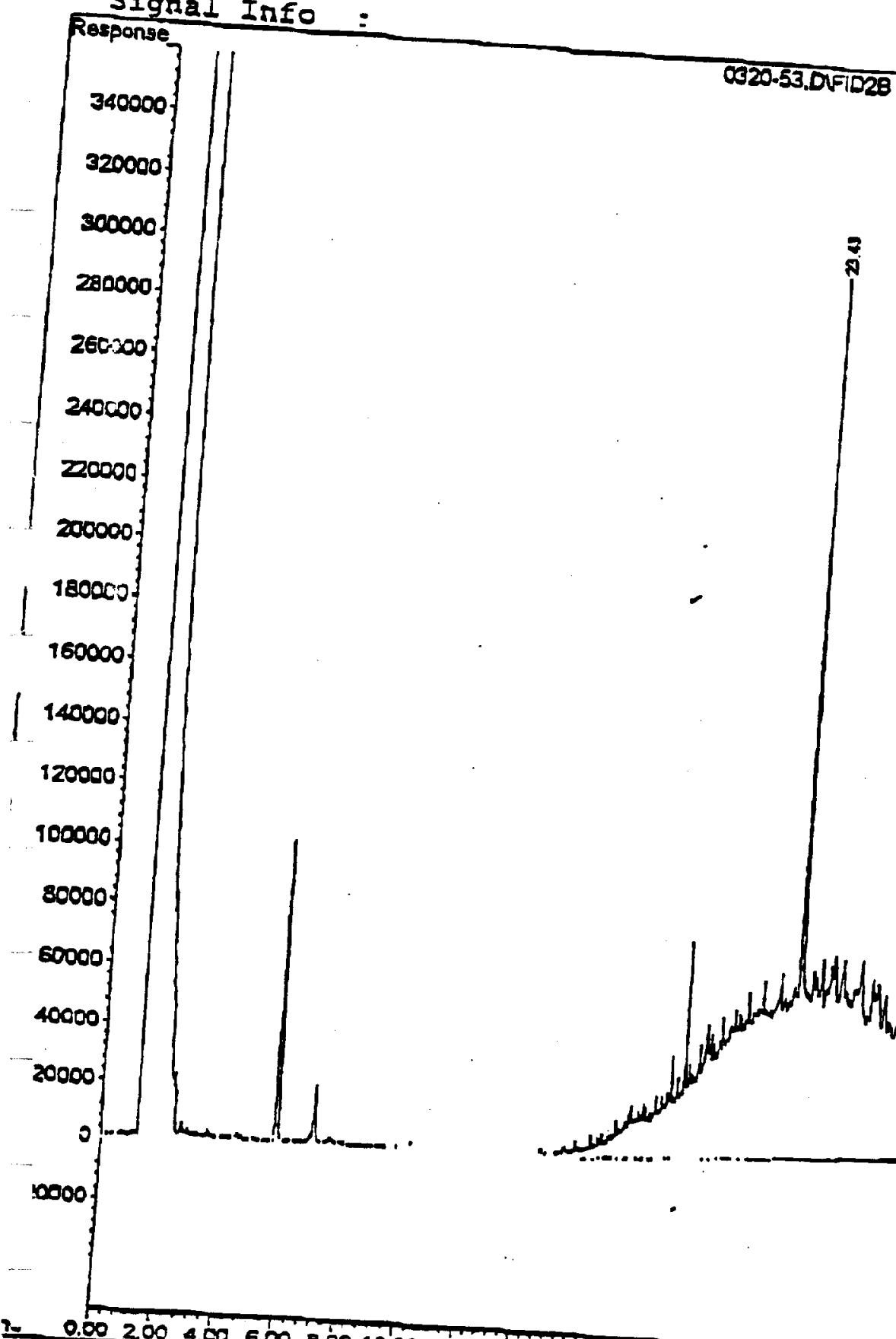

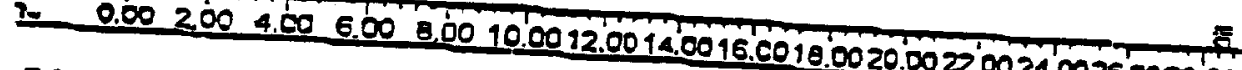

-3.D OIO101RI.M

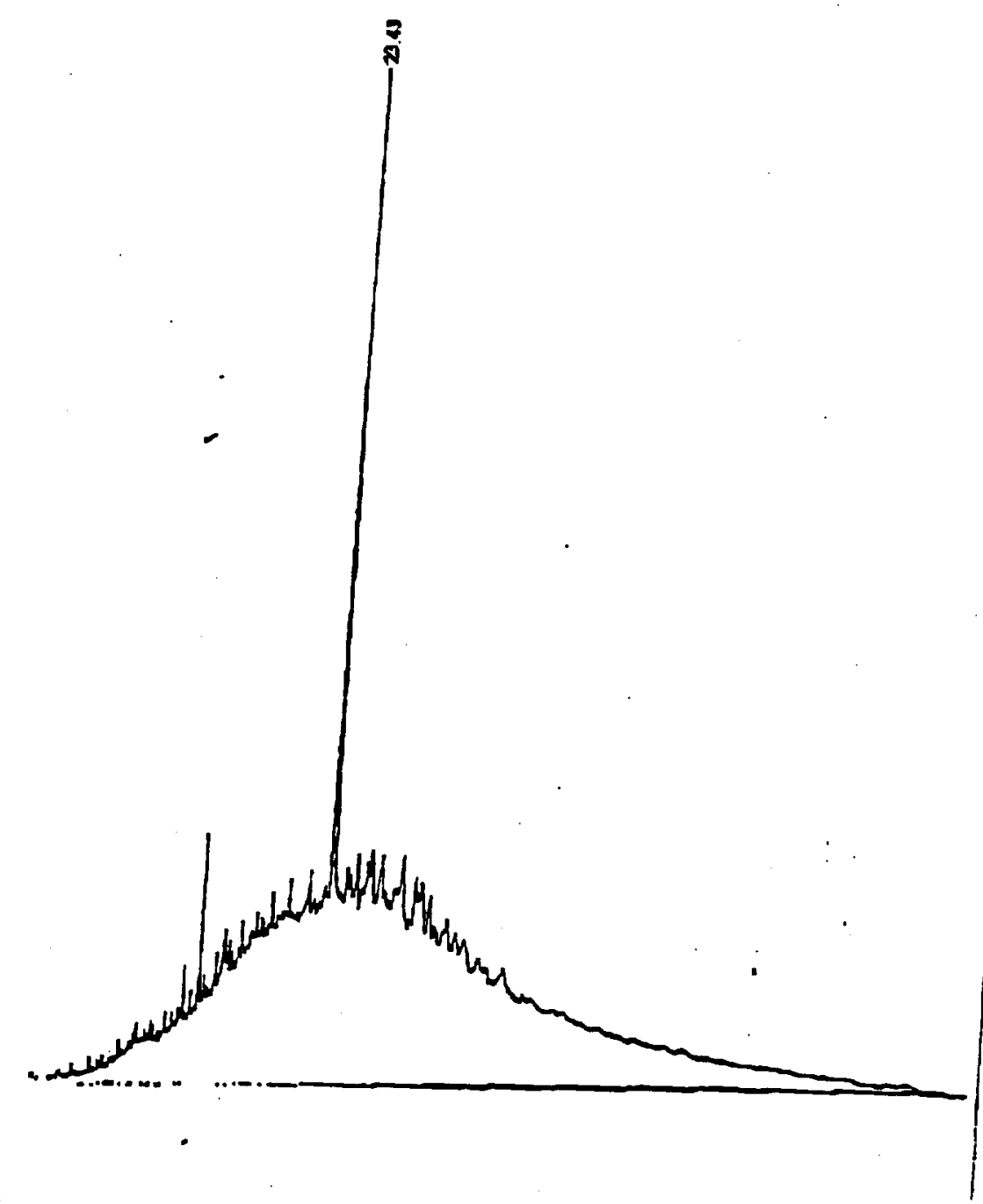

Operator: ecs

Inst: Seymour

Multipls: 1.00

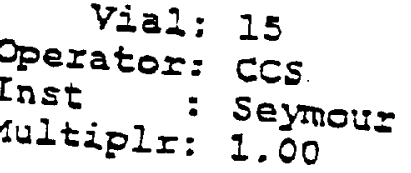




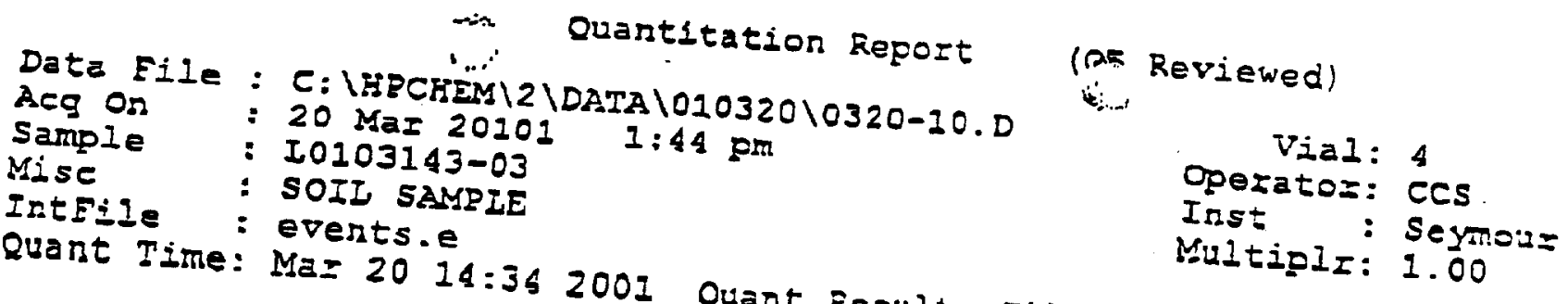

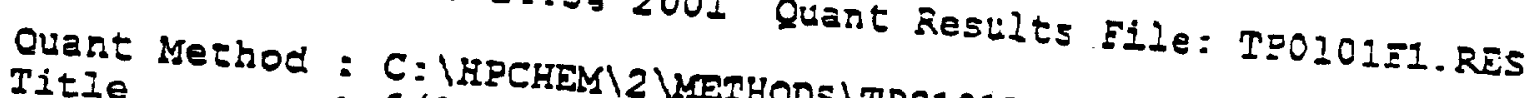

Last Update : 6/21/99 Seymour Eront IRH

Response via: Initial O5 08:51:13 2001

DataAcq Keth: TP9907RI.M

Volume Inj. :

Signal Phase:

Signal Info:

Compound

1) Target Compounds

?) H Gasoline

3) $\mathrm{H}$ Diesel
R. T

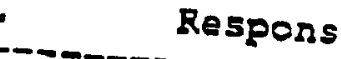

23.15

10.33

21.15
7525127

3190761

33523019
0.109

N.D.

N. D.
Cone taits

$\mathrm{mg} / \mathrm{ml}$

$m g / m i$ 


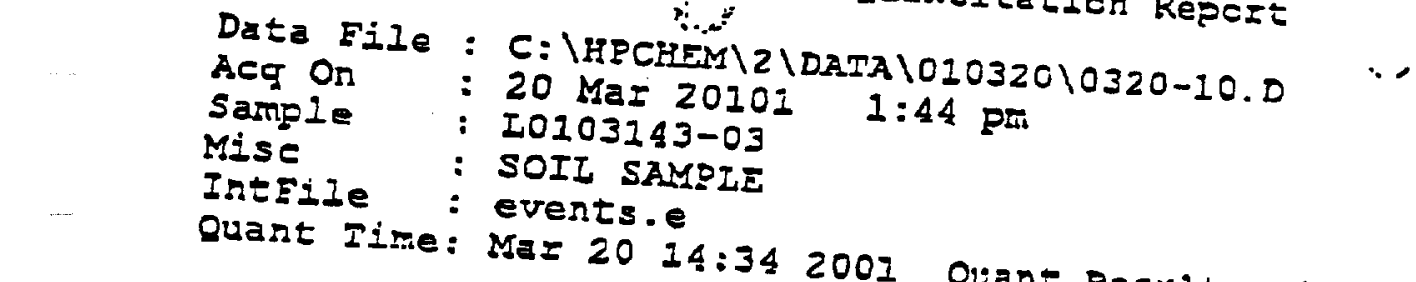

\section{Quantitacion Repcrt}

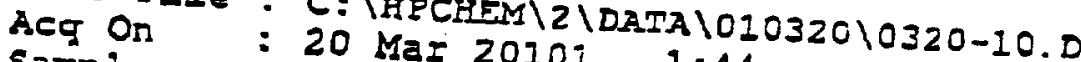

Mise : I0103143-03 1:44 Pa

InEFile : SOIL SAMPLE

Quant Iine: Mar 20 is.

Quant Method : C:IHPCHEMI2 METHODSITPOI0I
Title

Last Opdate: F/21/99 Seymour Ezont TPH

Resporse via : Multiple Jan 00:51:13 2001

Datascg Meth : TP9907RI.M

Volume Inj.

Signal Phase

Signal Info
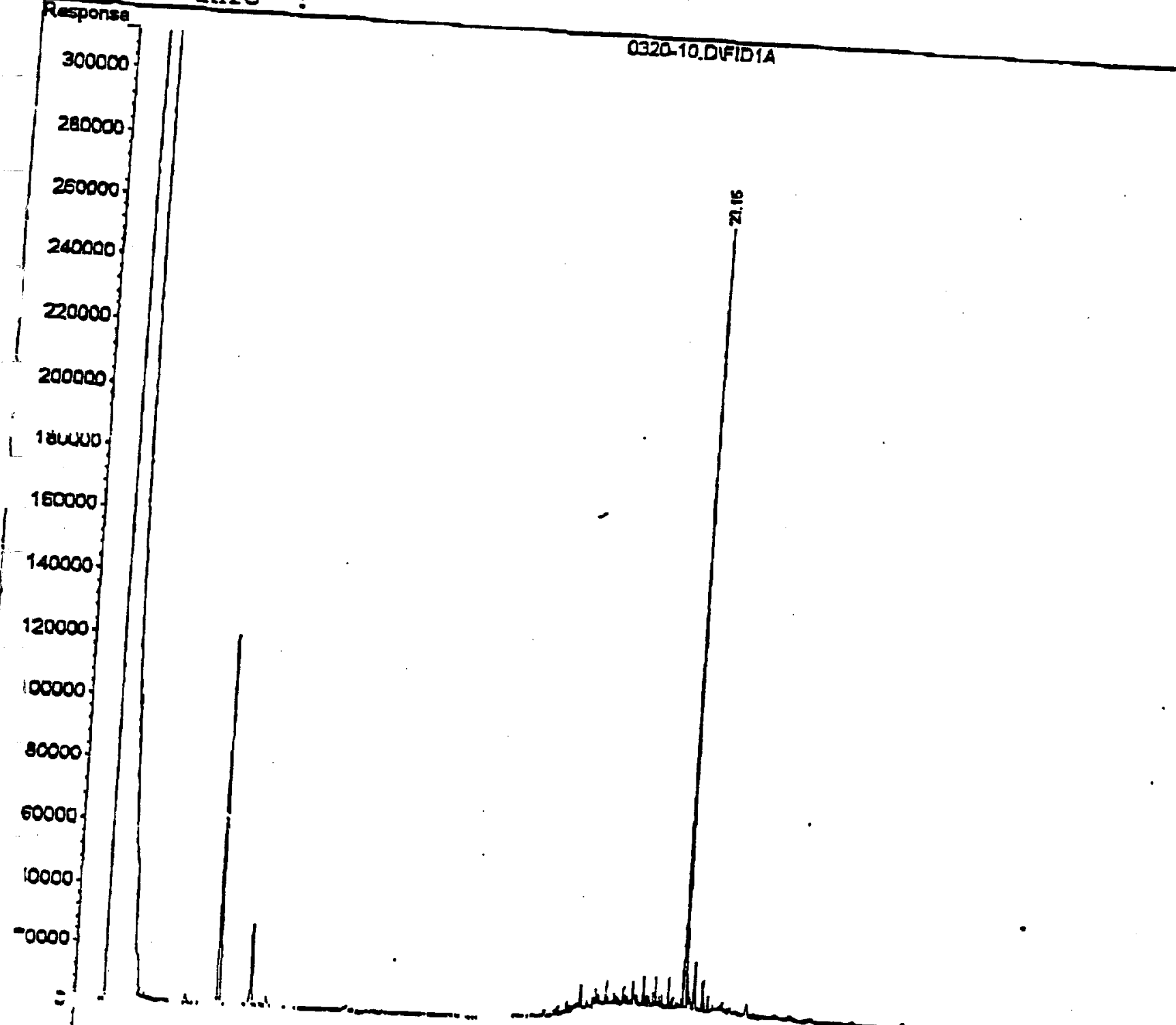

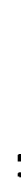

1000

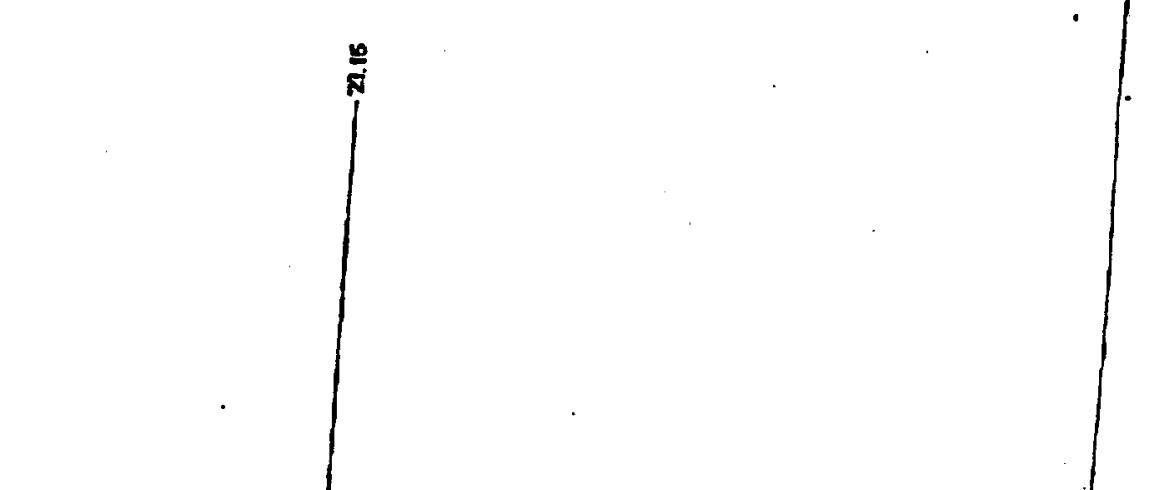

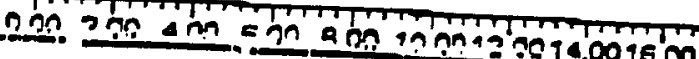

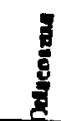

Operatoz: cas

Irst : Seymou=

Aultiplr: 1.00

V1ai:

Integrator) 


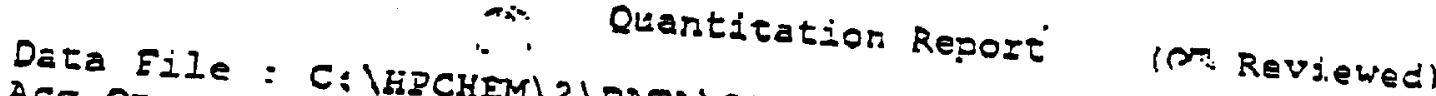

Acg on : 20 MaI 20101 DATA $1010320 \backslash 0320-18.0$

$\begin{array}{lll}\text { Sample } & : \text { LOI03143-04 } & \text { MaI } 4: 57 \mathrm{pm} \\ \text { Mise } & : \text { SOII }\end{array}$

IntEilo: SOIL SAMPIE

Quant Iine: Martis-e

01 Quant Results File: 0L001254.RES

Title

Last Opdate

Response via

DataAcq Meth

- 6/7 THCHEM IZ IMETHODS IOL0012F4

Fri Dec Seymour Exont Oil Method

Iritial Calibile:05 2000

TP9907RI.M

Volume Inj.

Sigral Phase:

Signal Info:

Compound

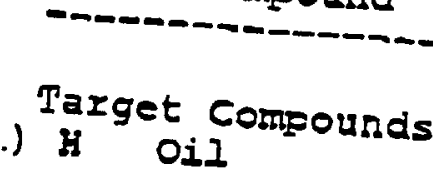

R.T

Response Conc Units

27.30

156382048

1.954

0.074

$\therefore 00$

Privis 36 ag

Vial: 12

Operator: CCS

Inst : Seymouz

Mulipl=: 1.00

$1.880 \times 1.0$

30.01 
-

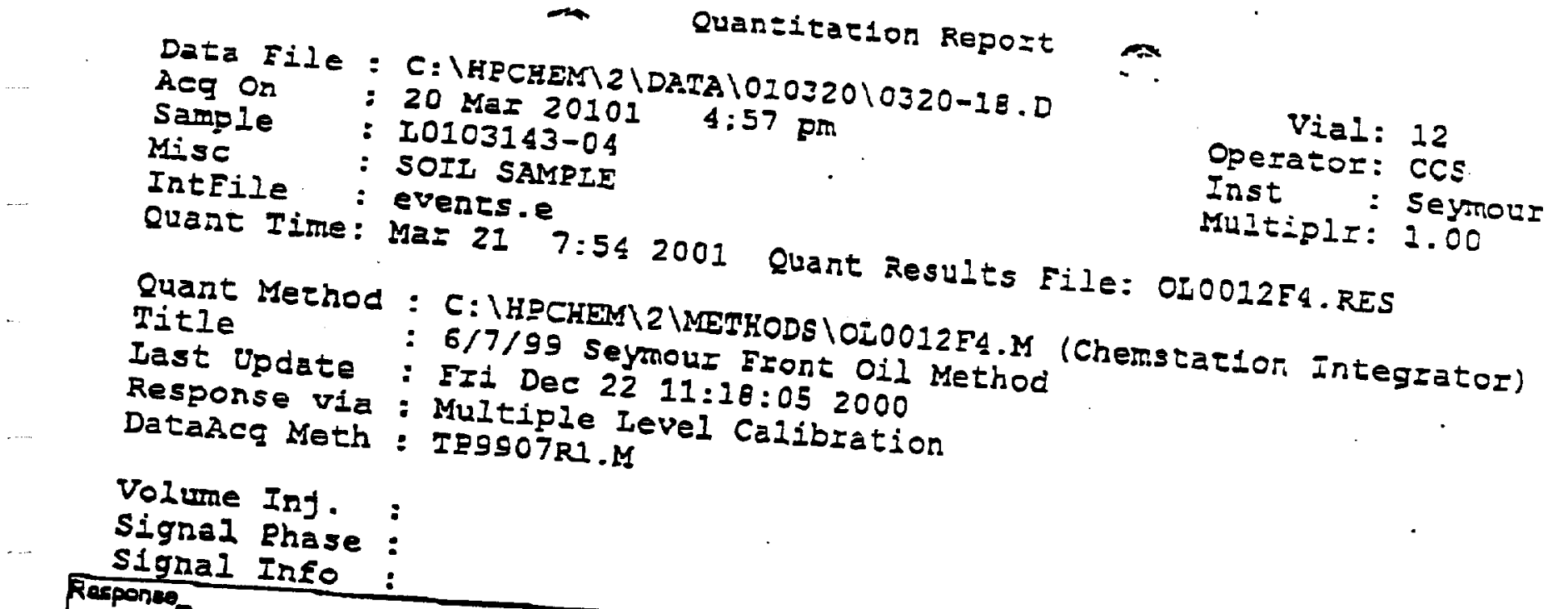
Data File = C: IHPCHEM 2 IDATA

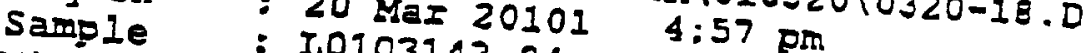
Misc : LO103143-04
IntEile : SOIL SAMPIE
Quant Time: Evars.e

Iitle

: 6/7/99

Response : FIi Dec 22 11:18:05 2000

DataAce Meth : TP9907Rl.M

Volume Inj.

Signal Rhase:

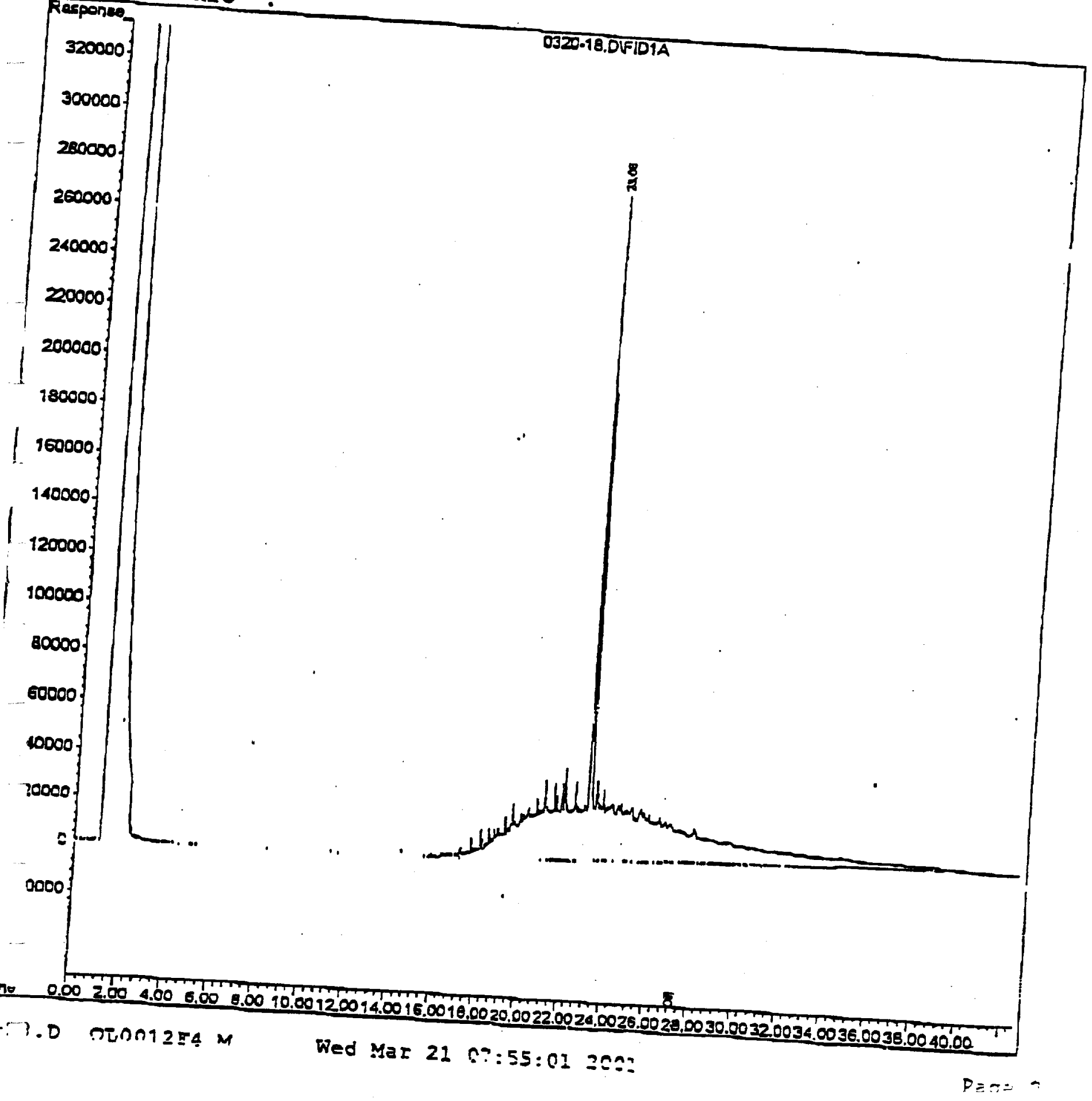




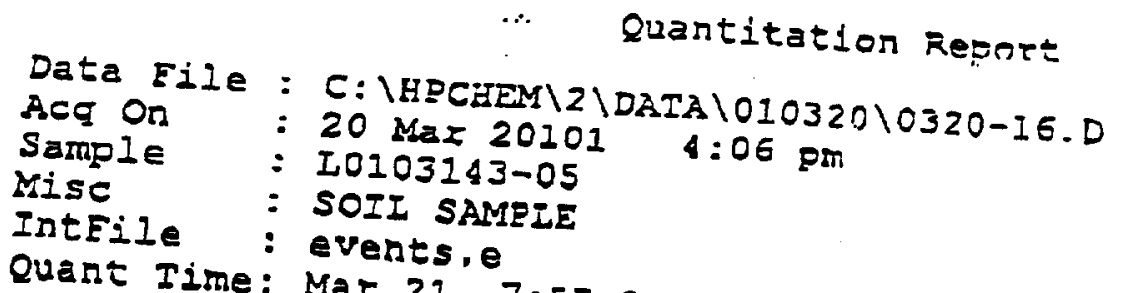

(mom Revietred)

$$
\begin{aligned}
& \text { Vial: } \text { lo } \\
& \text { Opezatoz: Ccs } \\
& \text { Inst : Seymeur } \\
& \text { Multipla: } 1.00
\end{aligned}
$$

Title

Last Update

Response via

DataAcq Meth

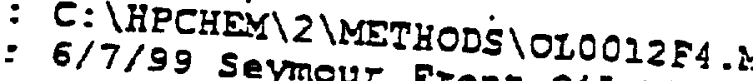

- Fris Seymour Front O1I Method

(Chemstasion Integrator)

Volume Inj.

Signal Phase:

signal Info:

Compound

- Insilal 22 11:18:05 2000

: IE9907Rl.M

L) Targer Compounds

R.T.

Response

Cone Units

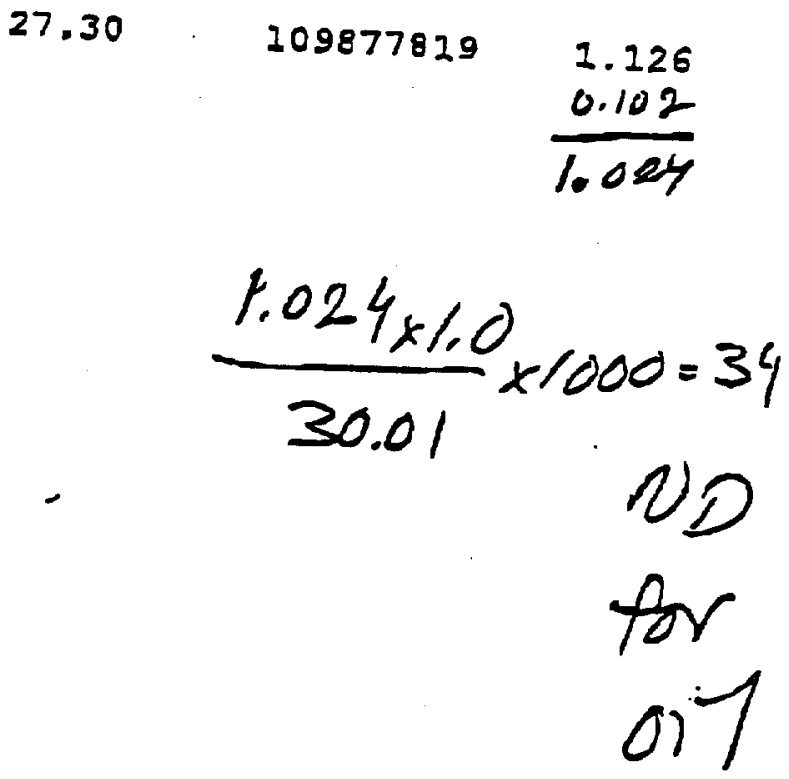


\%. Qusntitation Repozt

Data File : C:IHPCHEMI2\DATA

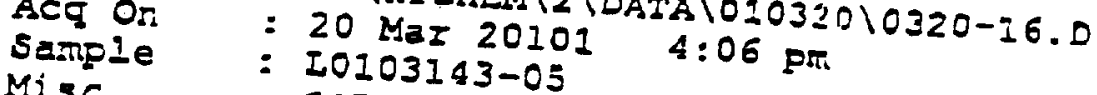

$\mathrm{Mis}$

IntFile: SOIL SAMPLE

Quant Time: Ments.e

Quant Resules Eile: OLO0I2FA.RES

Iitle

: 6/7/99 Soymour Fod LI001254.M (Chenstation Integrator)

Iast Opdate : Fri Dec $2211: 10: 05$ Oil Metrod

Response via : Multiple Level 18:05 2000

Datalco Meth : TP9907RI.M Level Calibrazion

Volume Inj. :

Signal Pinase:

Signed Info:

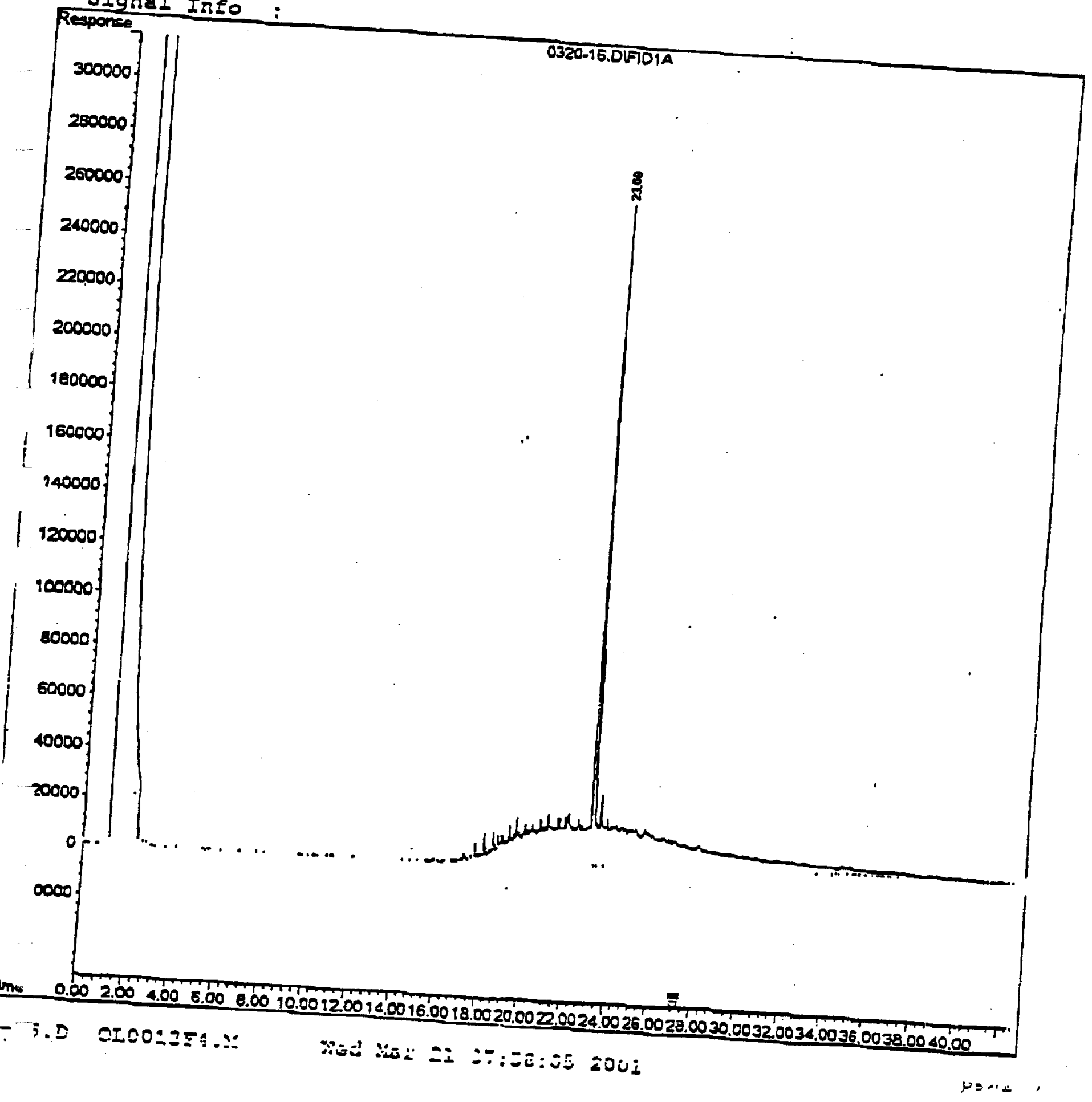




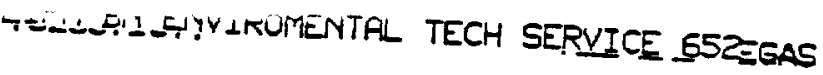

P.P. 13/3E

$$
\begin{aligned}
& \text { - Quantitarion keport (n? Revieves) }
\end{aligned}
$$

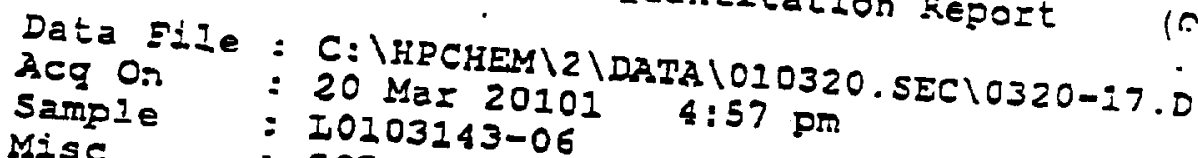

$$
\begin{aligned}
& \text { Mise : SOII SAMPIE } \\
& \text { IntFile: events.e } \\
& \text { Quant Fine: Mar } 21 \text { 8:01 } 2001 \text { Quant Results File: OLOLOLipir: }
\end{aligned}
$$

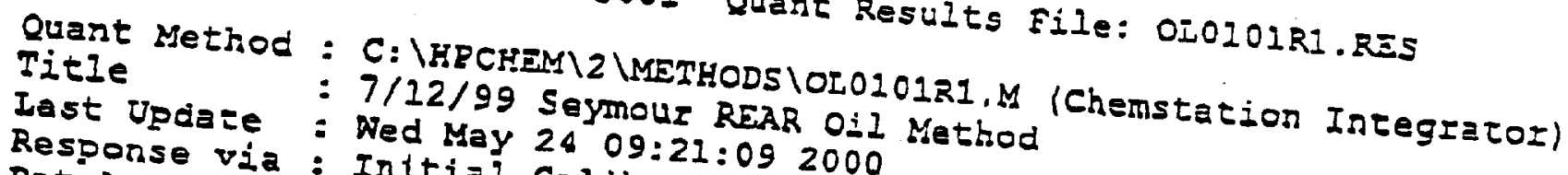

$$
\begin{aligned}
& \text { DataAcg Meth: InItial Calibration }
\end{aligned}
$$

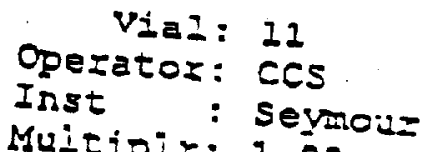$$
\begin{aligned}
& \text { Volume Inj. } \\
& \text { signal Phase: } \\
& \text { signal Info : }
\end{aligned}
$$

Compound

R.T.

$$
\text { Target Compounds }
$$

1) $\mathrm{H}$ 
Deta Eile Quartitarion Report

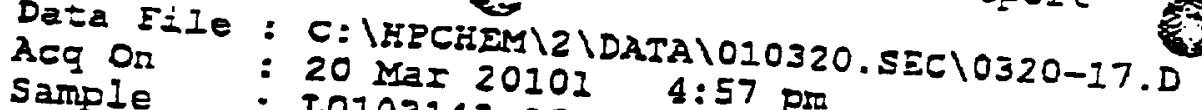

Sample : L0103143-06 $4: 57 \mathrm{pm}$

Mise

: SOII SAMPIE

IntFile : events.e

Quant Time: MaI 218.09

2001

Quant Method

Iitle

Iast Upciate

Response via

DataAcq Math

: C: IHPCHEM 2 LMETHODS IOLO101R7

Vial: 11

Operazor: cCs

Inst

: Setrmour

Mulipip: 1.00

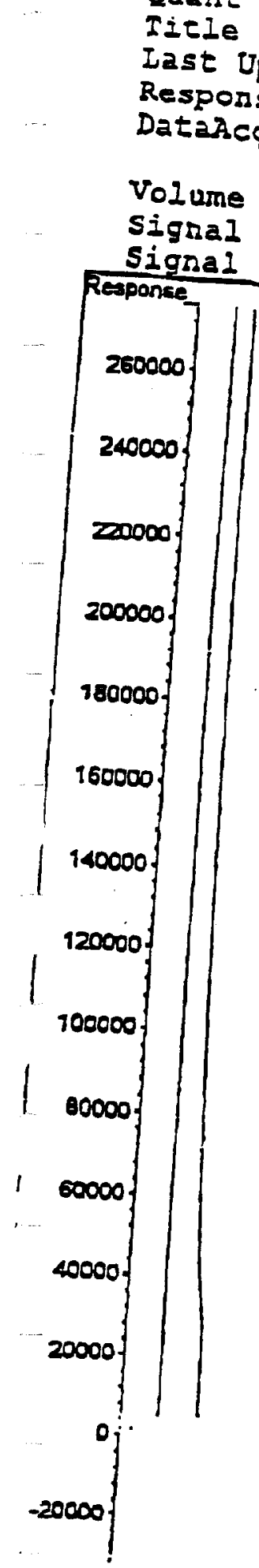

: 7/12/99 Seymour REAR OLO101RI.M

: Wed May 24 09:21:09 2000

: Multiple Level Calibration
T29907Rl.M

Volume Inj. :

Sigral Phase:

Signal Info:

$260000\} / \mid$ 0320-17.0151020 
Quantizazion Report (An Reviewed)

$\begin{array}{lll}\text { Acg on } & : 20 \text { MaI 2010I } \\ \text { Sample } & : \text { I0103143-06 } & \text { :57 pm }\end{array}$

Mise: SOIL SAMPIE

IntFile : events.e

Quant Time:

MaI 21

Quant Metiod :

Title

Last Opdate

Response via

Datascg Meth.

C:IHPCHEM 21 Magnt Results EIle: TF0101R2.RES

Vial: 1i

Cpezator: CCS

Inst : Seymo:=

Muitipla: 1.00

Volume Inj. :

Signal Rhase:

signal Info:

Compound

1)

Target Compounds

3) I Gasoline
R.T.

Response

Cone Units
7461677
918199
37848702

21.61
$0.111 \mathrm{mg} / \mathrm{mlm}$
$0.167 \mathrm{mg} / \mathrm{mI}$
$0.564 \mathrm{mg} / \mathrm{mI}$ ND 
Data File : Q Quasitation Repozt .

Aeg of : 20 MaI 20101

Samp: : : L0103143-06 \$:57 pm

IntFile : SOII SAMPLE

Quant Time: everts.e

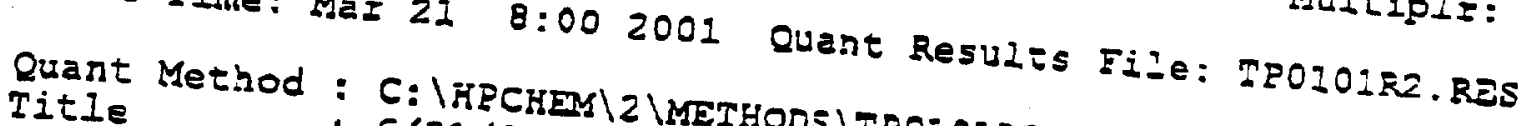

Last Update: Hed Jan 10 Seymour ExOnt TPH

Response via : Multiple Levei 10:47 2001

DataAcg Meth : Trg9o7Rl.M Lel Calibration

Volume Inj. :

Signal Phase:

Signal Info:

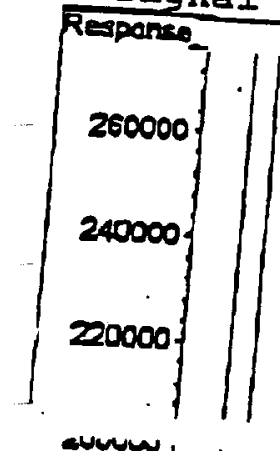

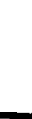




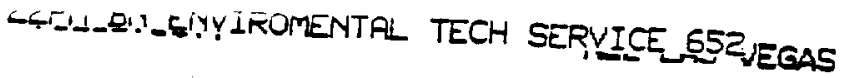

$P_{\text {Hive }} 1735$

$$
\begin{aligned}
& \text { Quantitation Report (AN Reviewed) }
\end{aligned}
$$

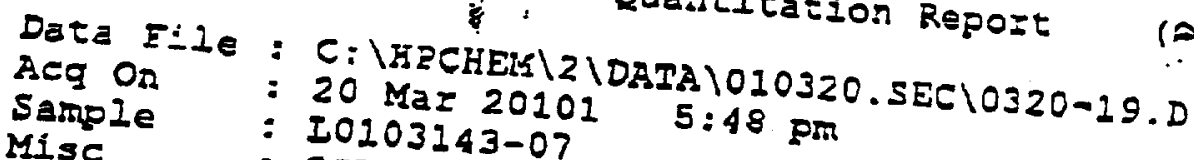

$$
\begin{aligned}
& \text { Misc : SOII SAMPIE } \\
& \text { Quant Time: events.e }
\end{aligned}
$$

2001 Quant Resits File: OLO101R1.RES

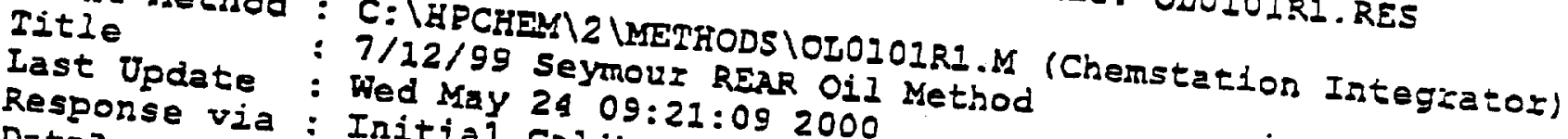

$$
\begin{aligned}
& \text { Response via : Wed May 24 09:21:09 2000 } \\
& \text { DataAcq Meth : Initial Calibration }
\end{aligned}
$$

Volume Inj. : Signal Phase:
Signal Info :

Compound

R.T.

Target compounds

1) $\mathrm{H}$ $0 \leq 1$
Response Cone Dits

$$
\begin{aligned}
& 28.53 \quad 112905186 \quad \begin{array}{l}
1.019 \\
0.103
\end{array} \\
& \hline 0.916
\end{aligned}
$$

$0.916 \times 1.0$

$$
\begin{aligned}
\frac{0.916 \times 1.0}{30.02} \times 1000= & 30.5 \\
& \text { No } \\
& \text { for } 0.1
\end{aligned}
$$

iT Delta > 1/2 N1.ndow

$-29 . D$ OLOIOIRI.M

Wed Mas 21 og:04:3e han. ixiginawual jut. 


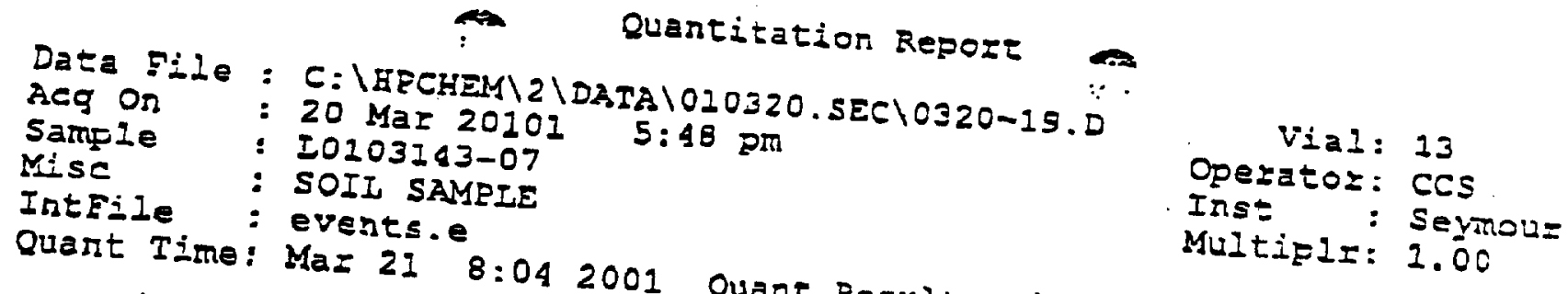

Quane Risults File: OLOIOIRI.RES

Ittie

: 7/12/99 SeymouTHODSIOLO101R1.M (Chemstation

Last Update : Wed May Seymour REAR Oil Method

Response via : Multiple 24 09:21:09 2000

Datasco Meth : TP9907RI.M

Volume Inj

Signal Phase:

Signal Info:

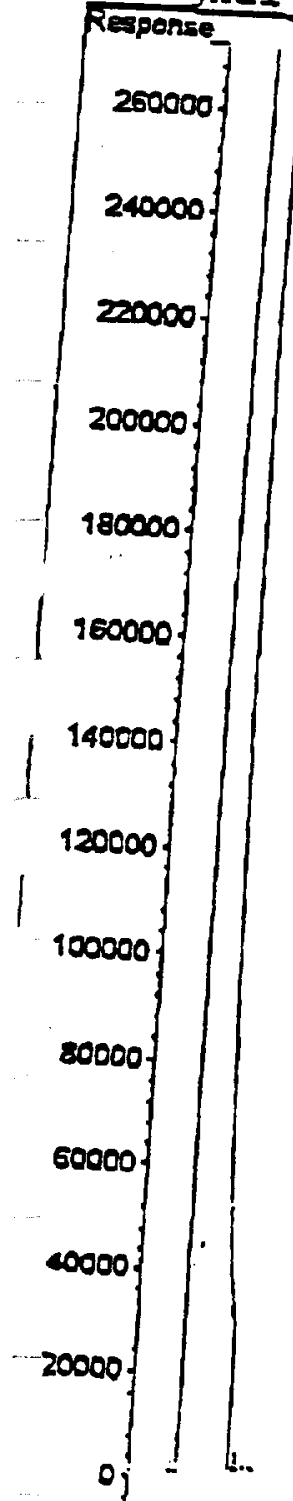

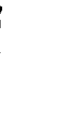




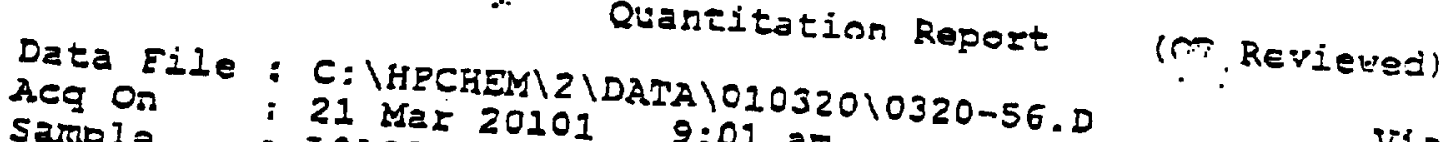

sample : 10203143-08 9:01 ar.

Misc : SOIL SAMP-O

V1a1: 16

IntFile : ever SAMPES

Quant Time: Marts.e

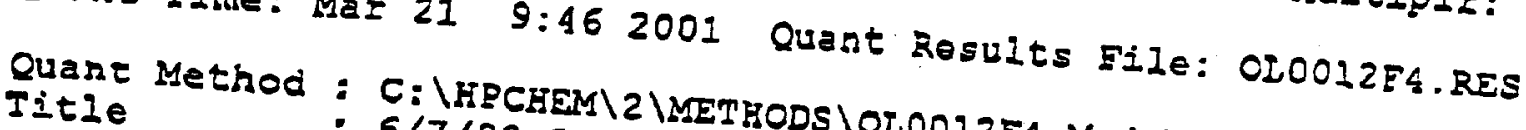

Last Update

Response vi

- 6/7/99 Seymour Front 0j1 Method (Chemstation Integrator)

1a: Initial 22 11:18:05 2000

Datacg Meth : Tp9907RI.M

Volume Inj.

Signal Phase:

Signal Info:

Compound

$$
\begin{aligned}
& \text { Operatoz: CCs } \\
& \text { Insz : Sevmour } \\
& \text { Multiplz: } 1.00
\end{aligned}
$$

\section{Compound}

Target Compounds
Oil

R.T.

Response Cone Dnits

27.30

$154044841 \quad 1.912$

0.101

$1.803 \times 1.0$

$30.09 \times 1000=60$ 
Data File : c:IFPCHEMII LATAl01032010320-56.0

$\begin{array}{ll}\text { Acg On } & =21 \text { MaI 20101 DATA } 101032010520-56.0 \\ \text { Sample } & : \text { LOI03143-08 }\end{array}$

Misc

InTEIIE: SOII SAMPIE

Quant Tine: events.e

$T 1 \leq I e$

6/7/g9EMI2 IMETHODS IOLO022F4.M (Chemsta

Iast Update : 6/7/59 Seymour Front Oil Method (Chenstatior Integrator)

Response via : Mul Dec 22 11:18:05 2000

DataAcg Meth: Trg907Rl.M Lel Calibzation

Volume Inj.

Signal Phase:

SignaI Info:

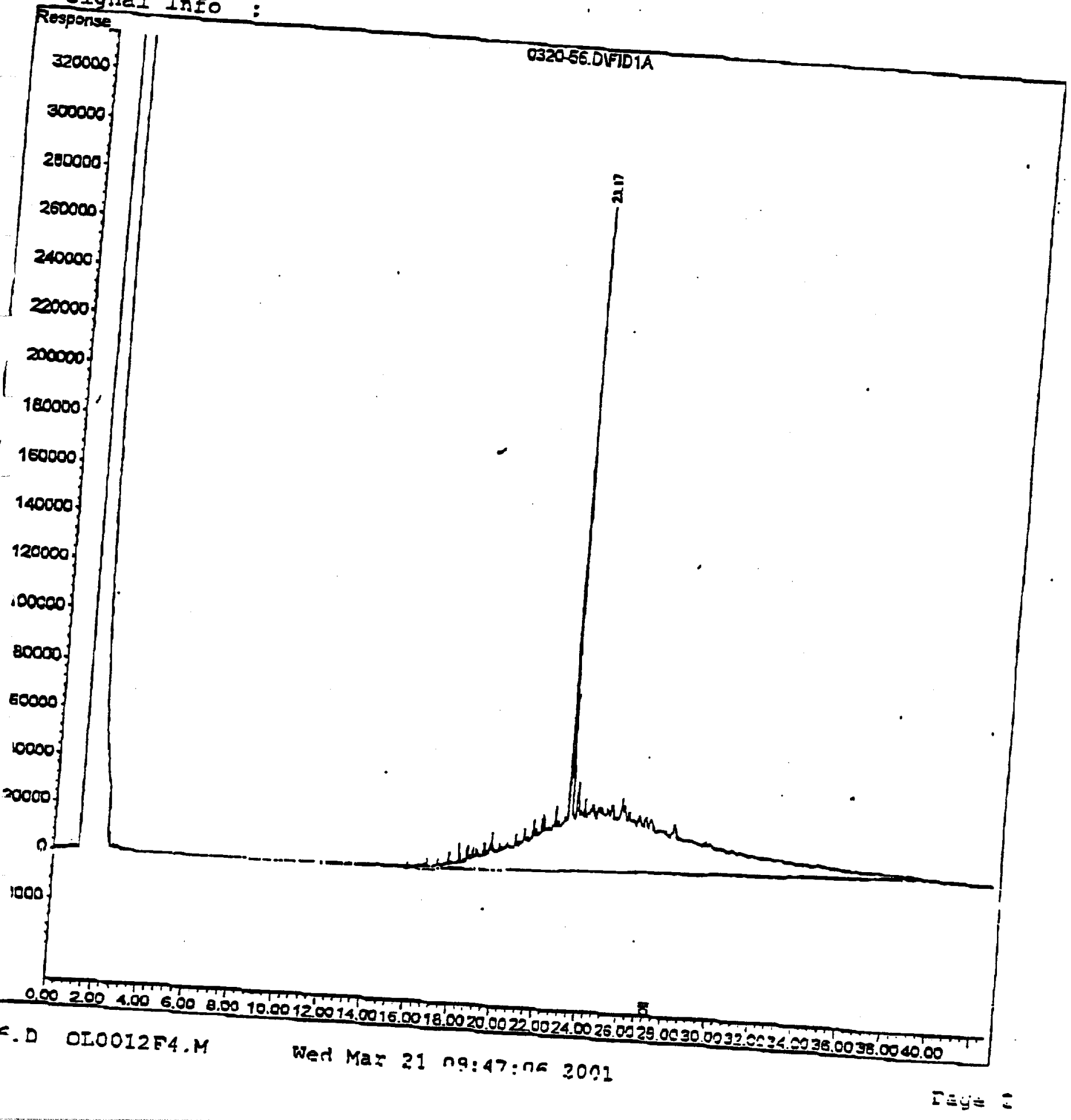


-- -

PP. 21/35:$$
\text { - Quantitation Report (ra Reviewed) }
$$

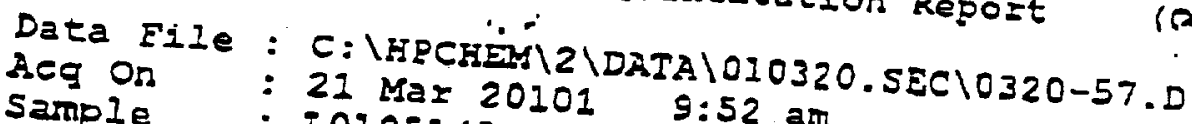$$
\text { Sample : L0103143-09 }
$$$$
\text { Mise : SOIL SAMPLE }
$$$$
\text { InterFile : events.e }
$$$$
\text { VIa: } 17
$$$$
\text { Operator: cos. }
$$$$
\text { Multiple: } 1.00
$$

2001 Quant Results File: OLO101R1. RES Last Update : Wed May 24 09:21:09 Oil Method

Response via : Initial Calibi21:092000

DaraAcq Meth : Tpgo07 RI Calibration

Volume Ind.

Signal Phase:

Signal Info:

Compound

R.T. Response Conc Units

Target Compounds

i) OIl

28.53

433351114

$$
\frac{6.250}{0.061}
$$

$\frac{6.189 \times 1.0}{29.96} \times 1000=207=210$

29.96

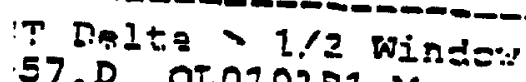

57.D OLOIOIR1.M

Wed Mar 21 10:40:50 na.

lii Häliudi init. 
C.. -

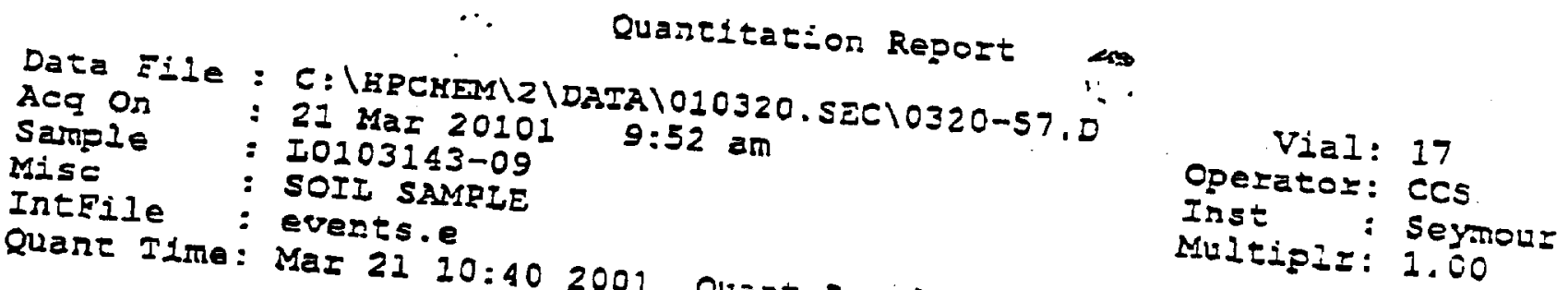

Quant Method : C:IHPCHEMI2|MEIHODSIOIOIOIRI

Last Update: W/12/99 Seymour REAR Oil Method

: 7/12/99 Seymour REAR Oil
Last Update : ied May 24 09:21:09 2000
Response via : Multiple Level Cal1bzation
DataAcq Meth : TP9907RI.M

Volume Ing.

Volume Inj. :
Signal Phase:
Signal Info :

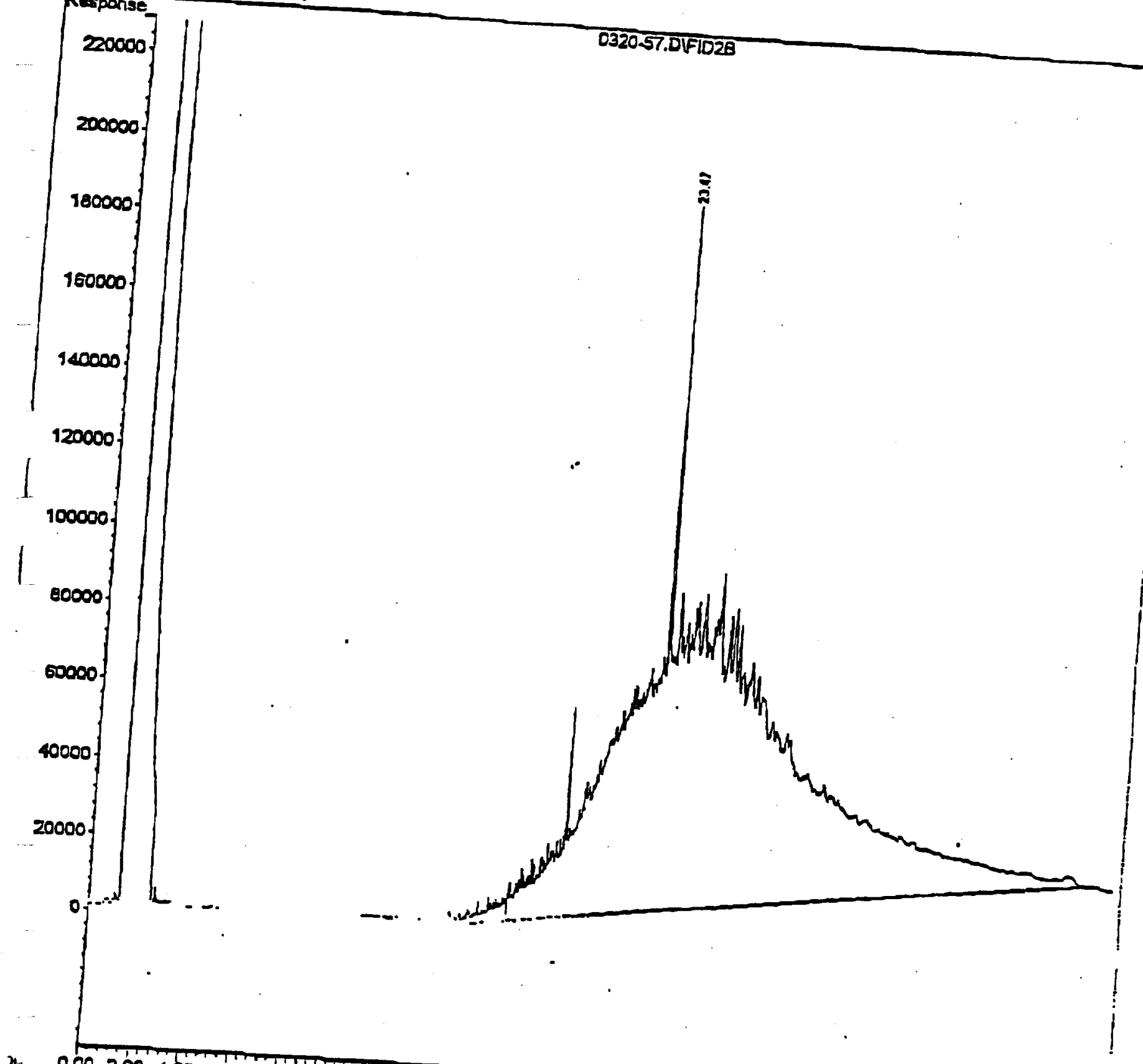

$x \quad 0.002004 .006 .008 .0010 .0012 .0044 .0016 .0018 .0020 .00270024 .0027$

$\rightarrow$. D otn1018.?.M

MIE: MII: :2: : : :15:50 2001 


\section{- -. - U.}

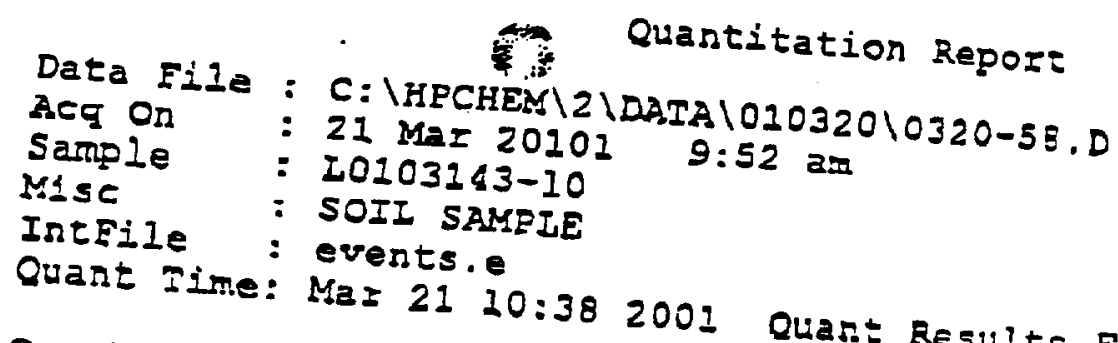

Quantitation Report

Acg on

Quan: Results File:

$$
\begin{gathered}
\text { Vial: } 18 \\
\text { Operator: CCS } \\
\text { Inst : Seymour } \\
\text { Multiplz: } 1.00
\end{gathered}
$$

Titie

Last Update

Response via

DataAcg Meth.

- 6/7/99 SEymoveTHODS LOLO012F4.M (Chemstesion

- Eri Dec 2211.18 .05 Oil Method

Initial Calibration 2000

TP9907R1.

Volume Inj.

Signal Phase:

Sigral Info :

2) Target Compounds

Compound

R.T.

Response

Cone Units

27.30

587267465

$\frac{9.758}{0.095}$

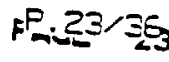

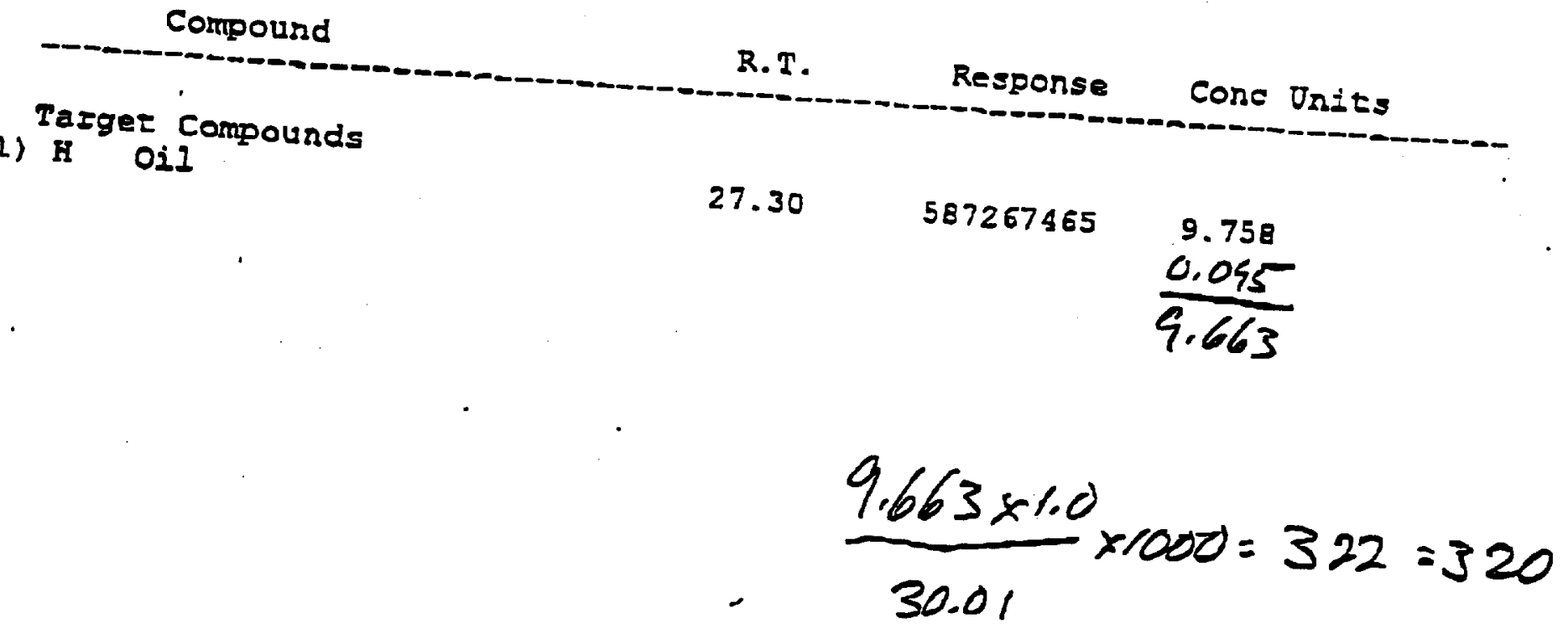




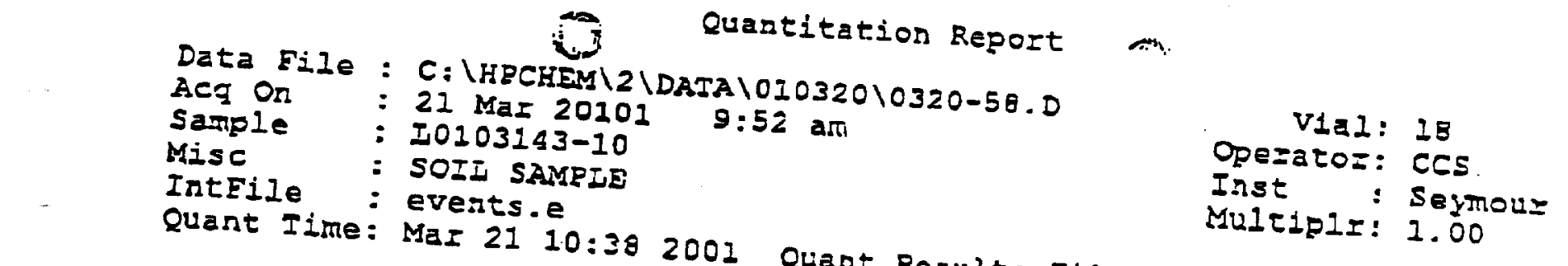

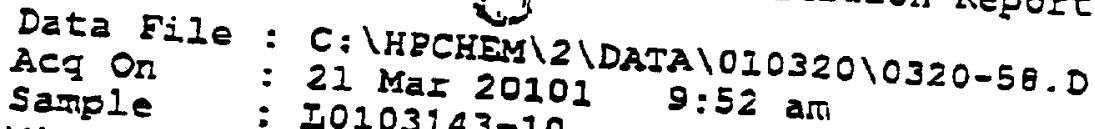

Misc :

: CCS

IntEile: everts

Quant Method: C:lHPCunMla

Title

: 6/7/99 SEymour Fron

Iast Update : FI Dec 22 mour Front Oil Method

Response via: Multiple 22 11:18:05 2000

DataAcq Meth : TP9907RI.M

Volume Inj. :

Signal Phase:

Signal Info

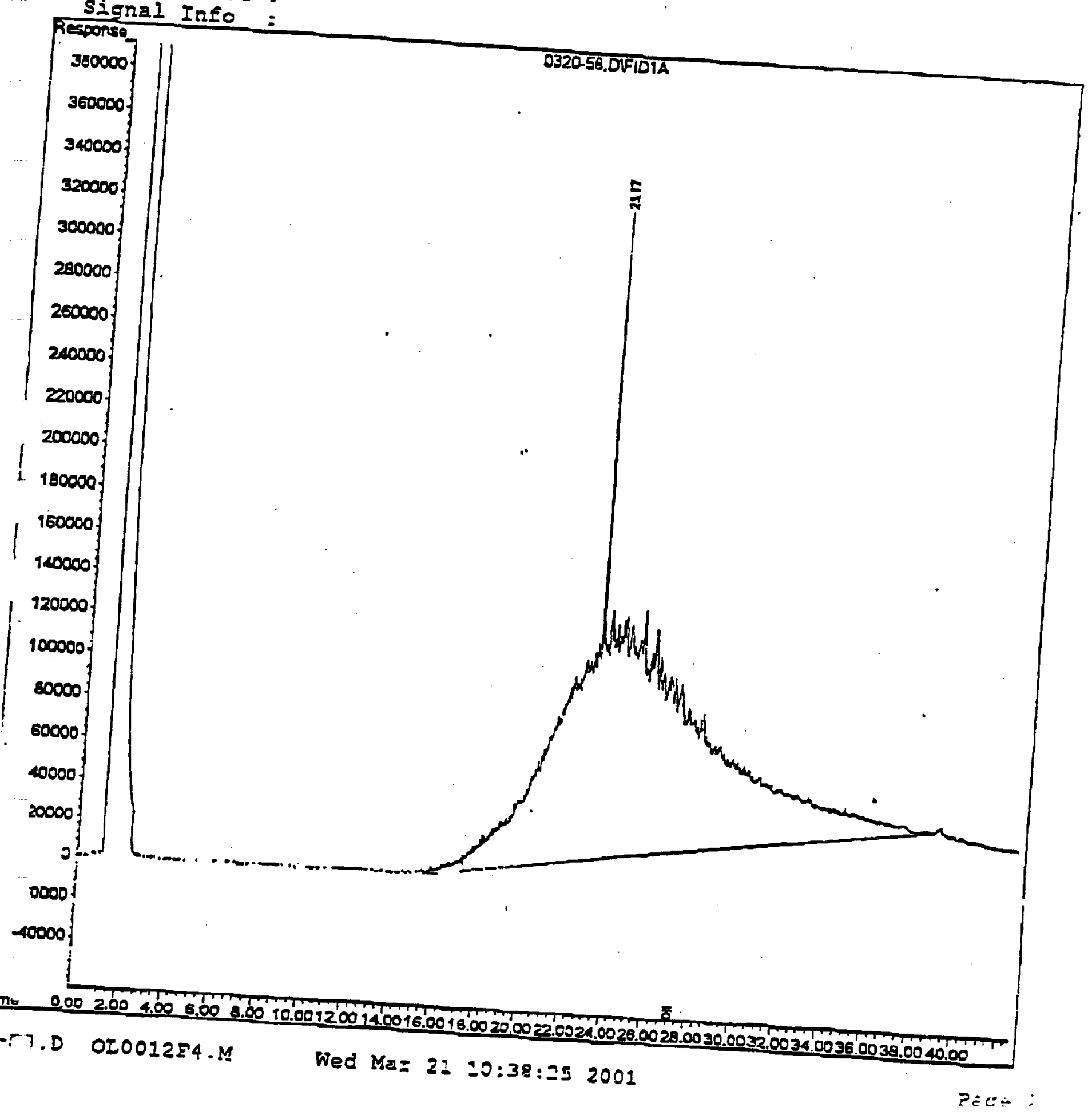




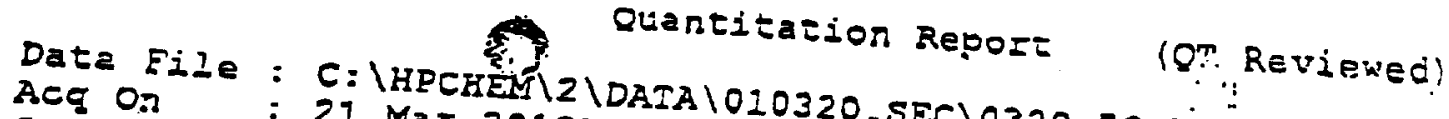

Acg OA : 21 MaI 20101 10.43 amo.sECI0320-59.D

Mise : I0:03143-11

IntEIle: SOIL SAMPIE

Quant T1me: events.e

Quant Method 21 1:28 2001 Quant Results File: OLOI01E..EEs

Titie

Iast Update

Response via

DataAcg Meth

Volume Inj.

Signal Phase:

Signal Info:

Compound

1) Harget Compounds

Target

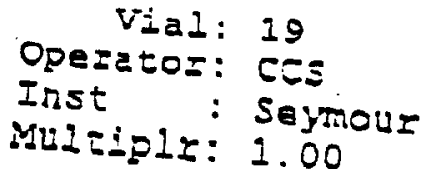

7/12/99 SEYMOUHODS OOLO101RI-M (Chemstation Integretor)

Wed May 24 09:21:09 OII Method

Initial Calibration 2000

TP9907Rl.M

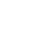

$28.53 \quad 765368605 \quad \frac{21.670}{0.080}$
$\frac{11.590}{1150}$

$11.590 \times 1.0$

$30.04 \times 1000=385.8=390$ 


\section{$\because \because$ Quartitation Repors}

Data File : C:IHPCHEM 2 IDATAl010320.55Cl0320 On

Samplo : $21 \mathrm{Mat} 20101$ 10:43 am .5ECl0320-59.0

MIsc : L0103143-11

IntFile : SOII SAMPLE

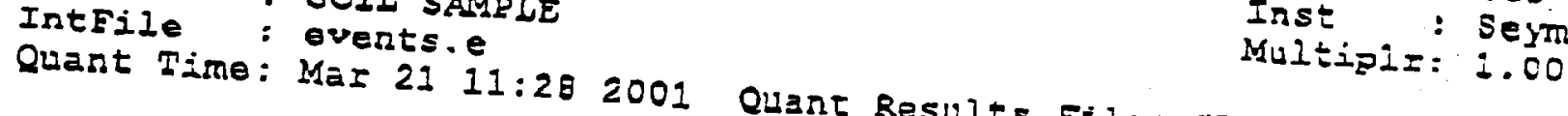

Vial: 19

Operator: eCS

Inst : Seymour

Multipla: i.00

Resties File: OIULi:Ẽ.RES

Title

Iast Update

: Wed May 24 09:21.09R Oil Method

DataAcg Meth : TP9gotR1.M

Volume Inj.

Signal Phase:

Signal Info

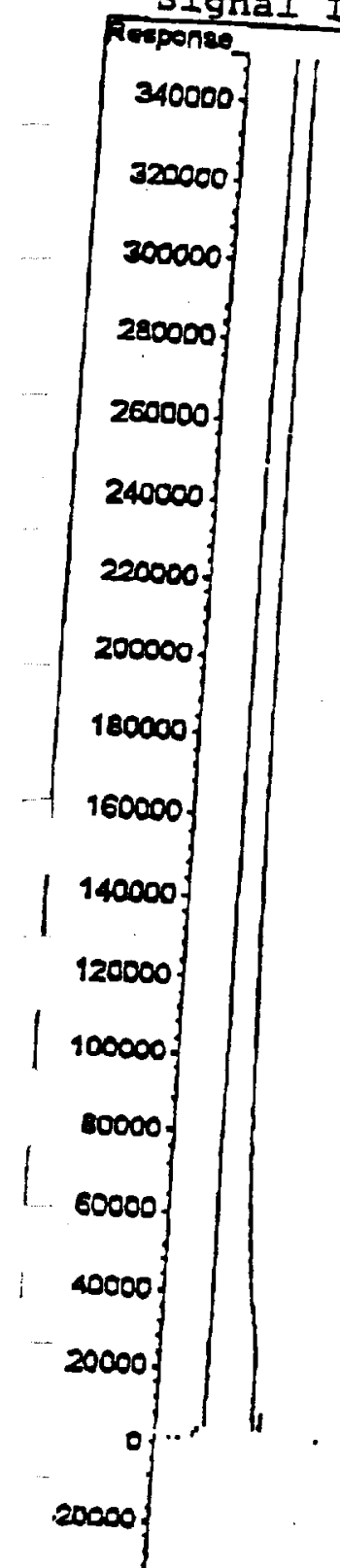

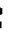
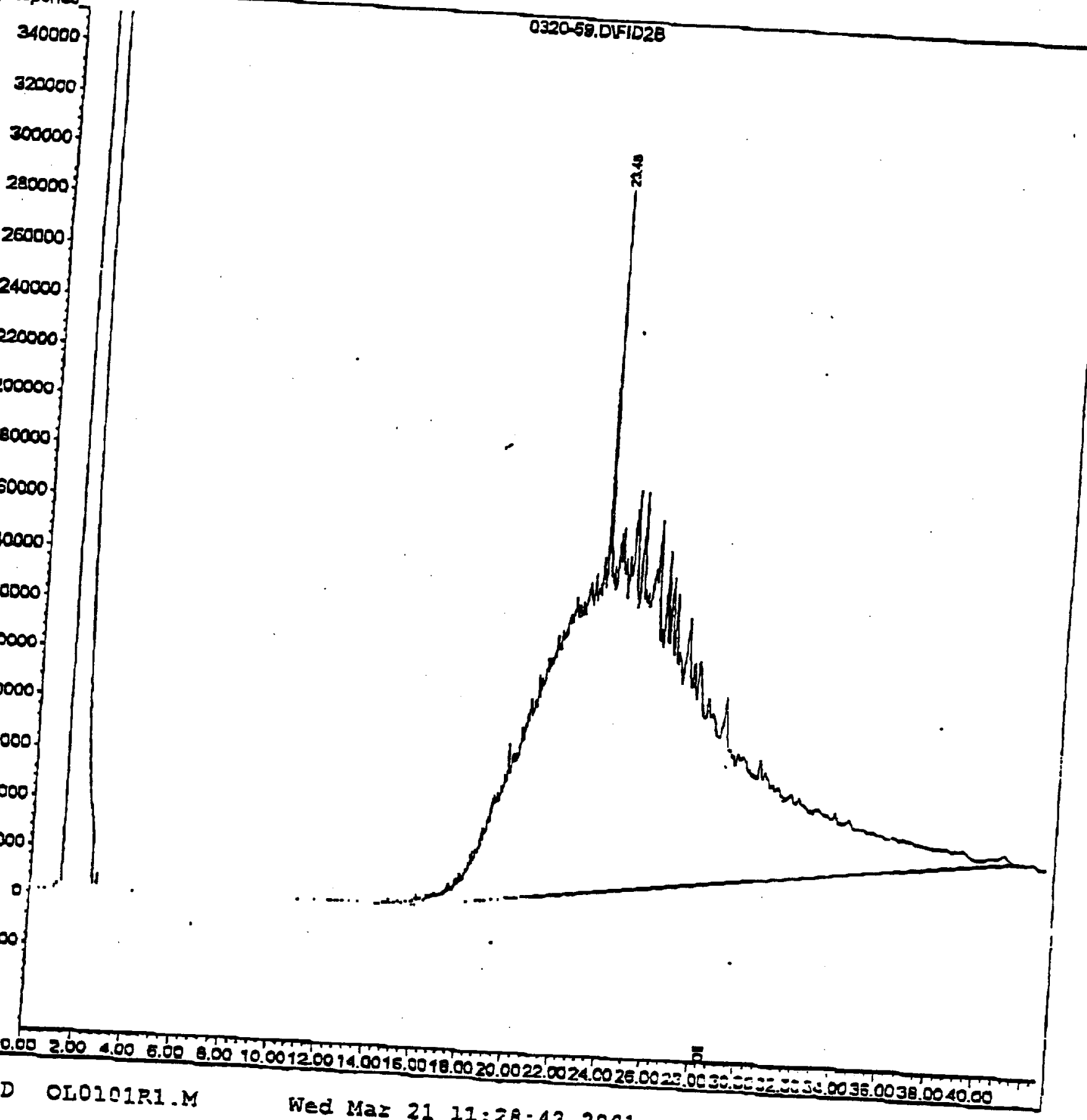
Data File: C:IHechem

Ace on : $20 \mathrm{Maz} 20101$ DATA

Sample : 20103143-12 $5: 48 \mathrm{pm}$

InTFile: SOIL SAMPIE

Ouant Time: events.e

Title

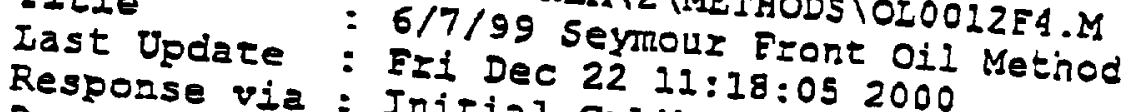

(Oriseviewed)

Datancq Meti : IP9907RI.M

Volume Inj.

Signal Phase:

Signal Info:

Compound

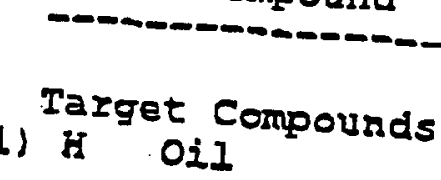

27.30

$82760462 \quad 0.644$

$$
\begin{aligned}
& \text { Vial: } 14 \\
& \text { Operatoz: CCS } \\
& \text { Inst : Seymour } \\
& \text { Multiplr: } 1.00
\end{aligned}
$$

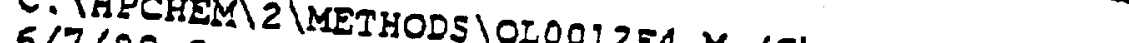

(Chenstation Integrator)

1) if oil

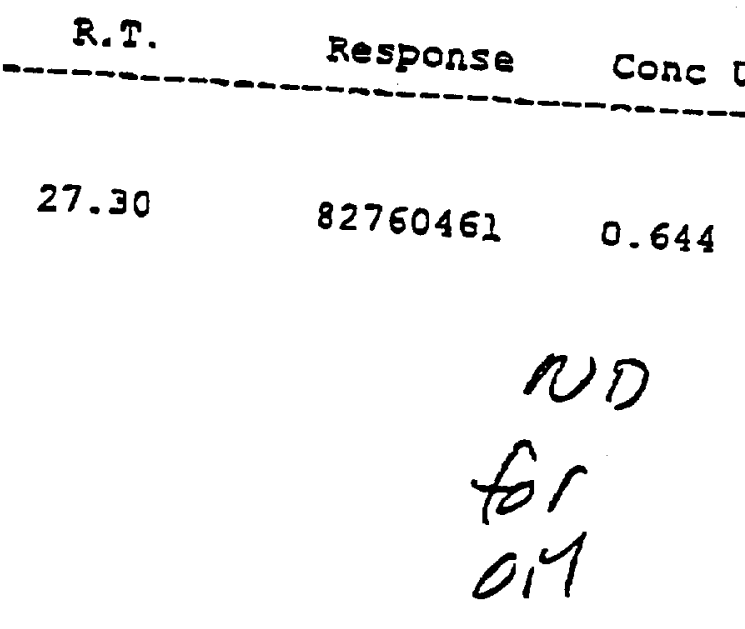




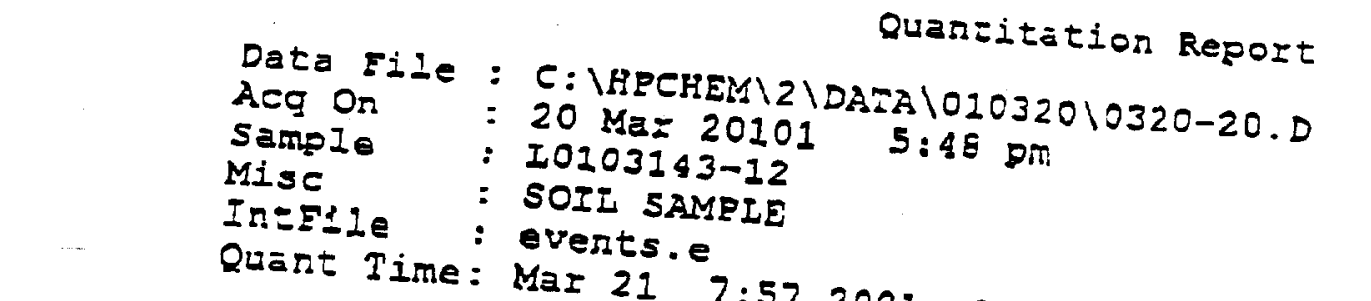

is:

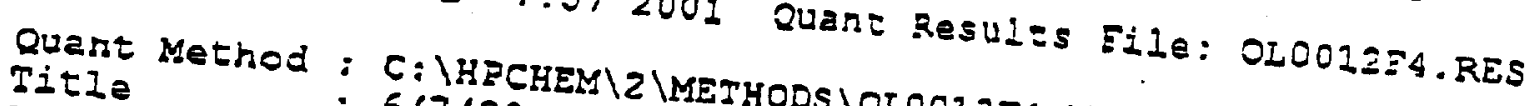

: 6/7/99 SELMETHODSIOLOO12E4.M (Chemstation IntegE

Last Update : Eri Dec Seymour Era:. Oil Metiod

Response via: Multiple 22 11:18:05 2000

Datakcq Meth : Tr9907RI.M

\section{Volume Inj. :
Signal Phase:
Signal Info:}

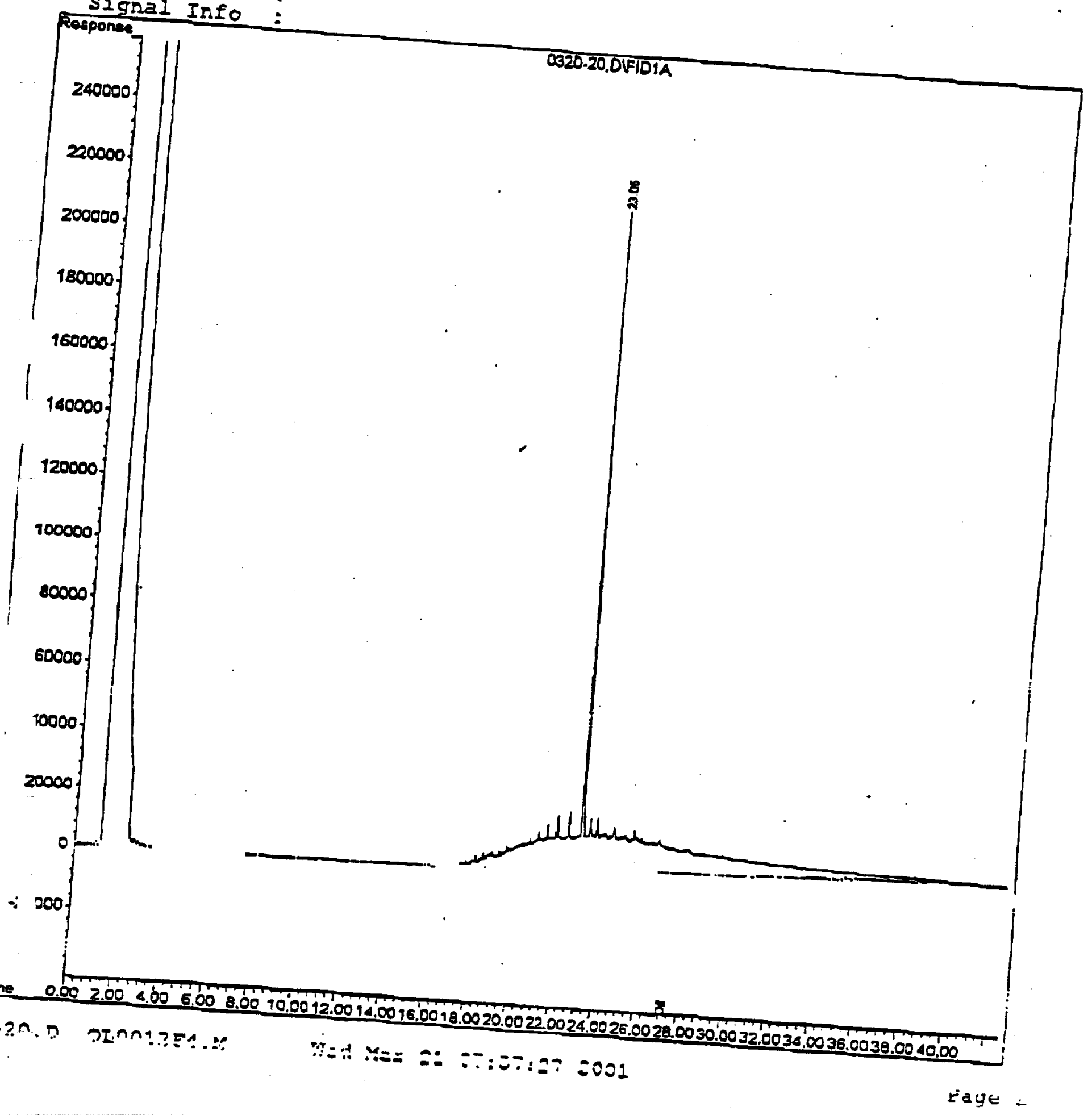




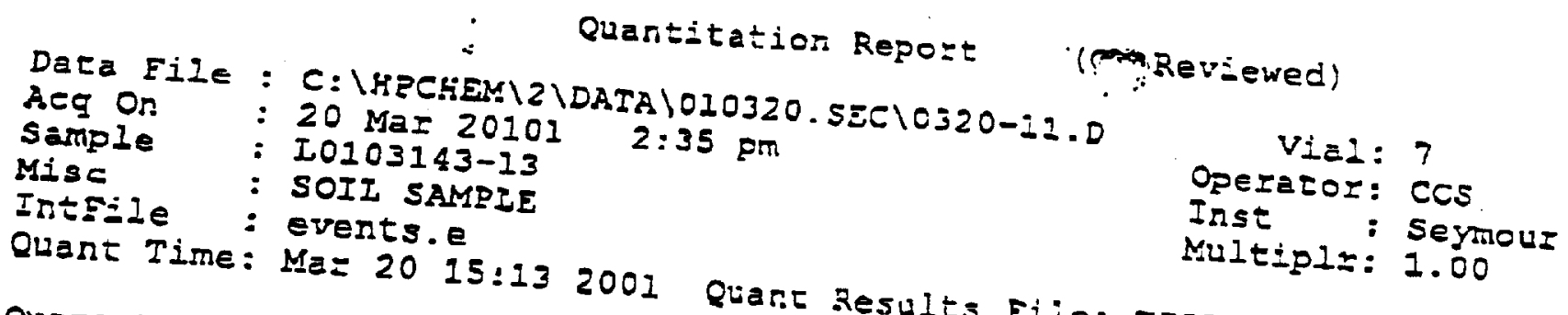

Oint Results File: TPOI01RZ.RZS Ticle 6/21/99 SEMETHODSITEO101R2.M (Chemstation Integrator)

Last Update: Wed Jan Seymour Ezon: TP:

Response via : Initial Ca 15:10:47 200:

Datara Meth : Tp9907RI M Mibrarion

Volume Inj.

Sigral Phase:

Signal Info:

Compound
1) Target Compounds
2) H Octacosane
3) H Gasoline
Diesel

R.I.

Response Conc Units

$\begin{array}{rrll}23.42 & 6296536 & 0.094 \mathrm{mg} / \mathrm{mlm} & \\ 11.70 & 465347 & 0.153 \mathrm{mg} / \mathrm{ml} & \\ 21.61 & 15147843 & 0.232 \mathrm{mg} / \mathrm{ml} & \text { ND }\end{array}$


Daca File : C: IHPCHEMI2

Sample : $20 \mathrm{Mat} 20101 \quad 2: 35 \mathrm{pm}$.SEclo320-11.D

Quastita
A.
20101
$43-13$

Mise : SOII SAMPIE

Inteile : events.e

Quant Time:

MaI 20 15:13 2001

Quant Method : C:IHPChEN Quant Results File: TRO10IRZ.RES

Title

Iast update: 6/21/99 Seymour Erent TFH
Response via: Wed Jan 10 15:10:47 2001

Datalicg Mela : Multiple Level ca:472001

6/21/SO

DataAcg Meth : TP9907RI.M

Viel: 7

Operator: cCS

Inst : Seymour

Multiole: 1.00

Volurae Inj.

Signal Phase:

Signal Info:

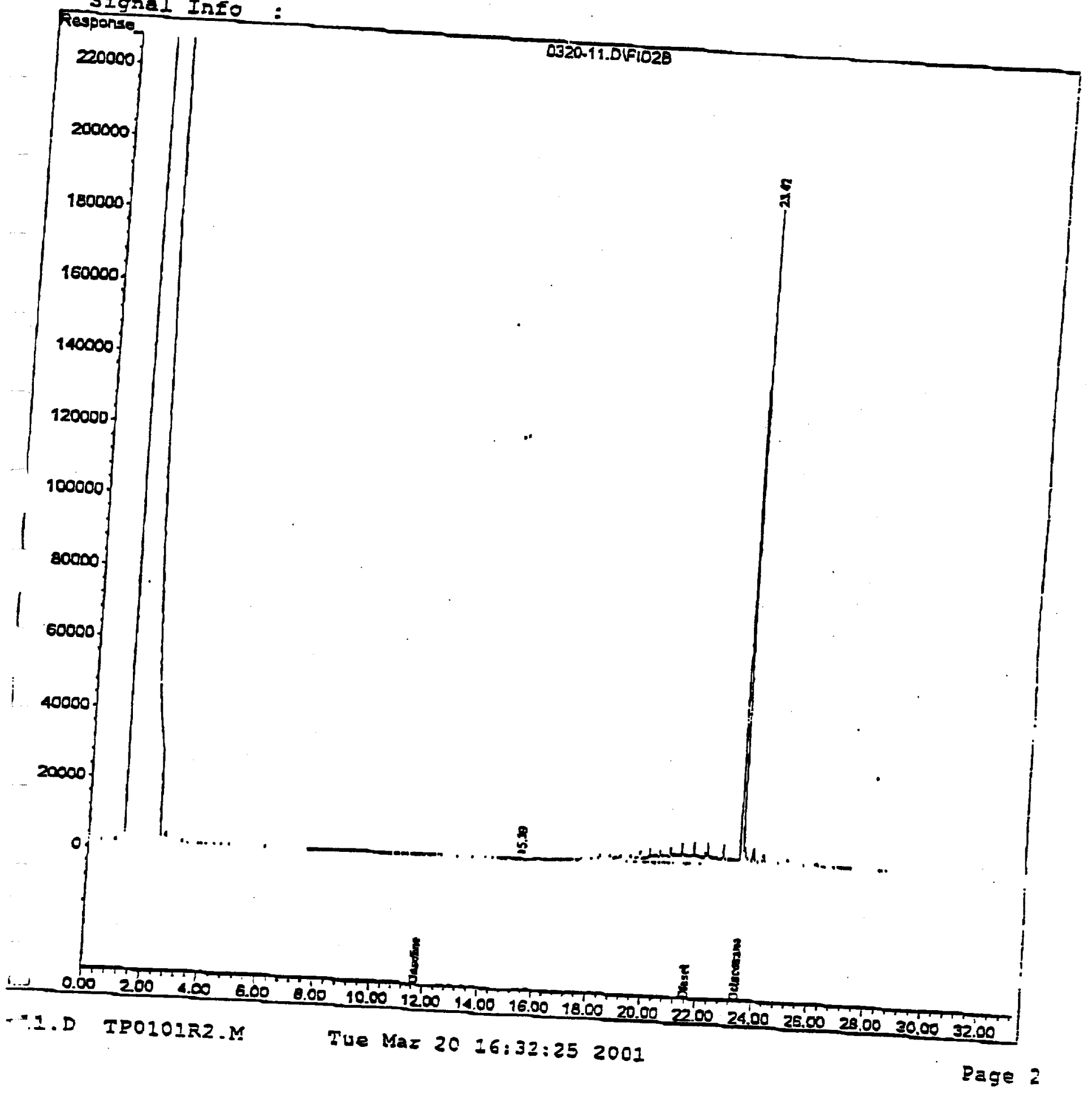


Data File : C:lypcreylat

Acg On: 22 Mat 20101 DATA $01032010320-60.0$

Sample: :0103143-14 10:43 am

Misc

IntFile : SOIL SAMELE

Quant Tine: events.e

Quant Results File: OI0012E4.REs

Iitle

Last update

Response via

DataAcq Meth:

6/7/99 SEYMOUETHODS \OLO012F4.

Fri Dec Seymour Esont Oil Mechod

Initial Calibiat:05 2000

TP9907R1.M

Volume Inj. :

Sigral Phase:

Signal Info:

Compound

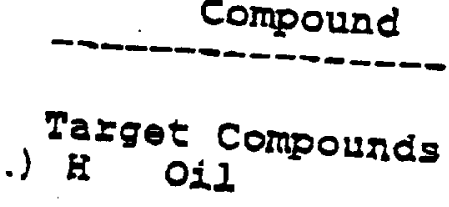

$27.30 \quad 483443333 \quad \frac{7.855}{0.092}$
$\frac{7.763}{3.763 \times 1.0}$
$\frac{30.02}{3} \times(000)=258.6=20$

R.T.

Response

Cone Units

Operator: 20

Inst

MuItipla: 1.00

Vial: 20

Reviewed)

Chemstation Integrator)

H Oil

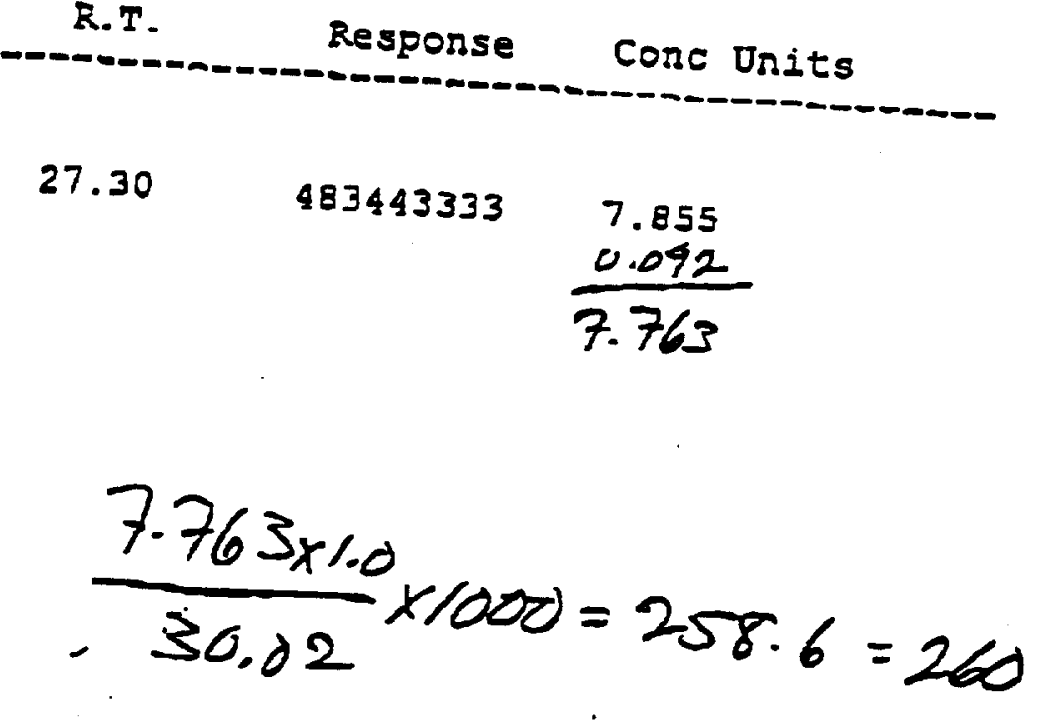


Data File : C:IHECHEMI2IDATA OUantitation Report
Acg On

Sample : I0103143-14 10:43 am

Mise : SOII SAMPIE

Ir.tFile : voents.

Quant Time: Masts.e

Vial: 20

Operatcr: cCs

Inst : Seymoliz

Mutiple: 1.00

Quant Results File: OLOOL2F4.RES

Tivie

Last Update

- Fri Dec 22 ymour Ezos: 01I Method

Datponse oia : Muliple Ievel Calibration

: 6/7/99 Seyour

Datascq Meth : TP9907RI.M

Volume Irj. :

Sigral Fhase:

slgral Info:

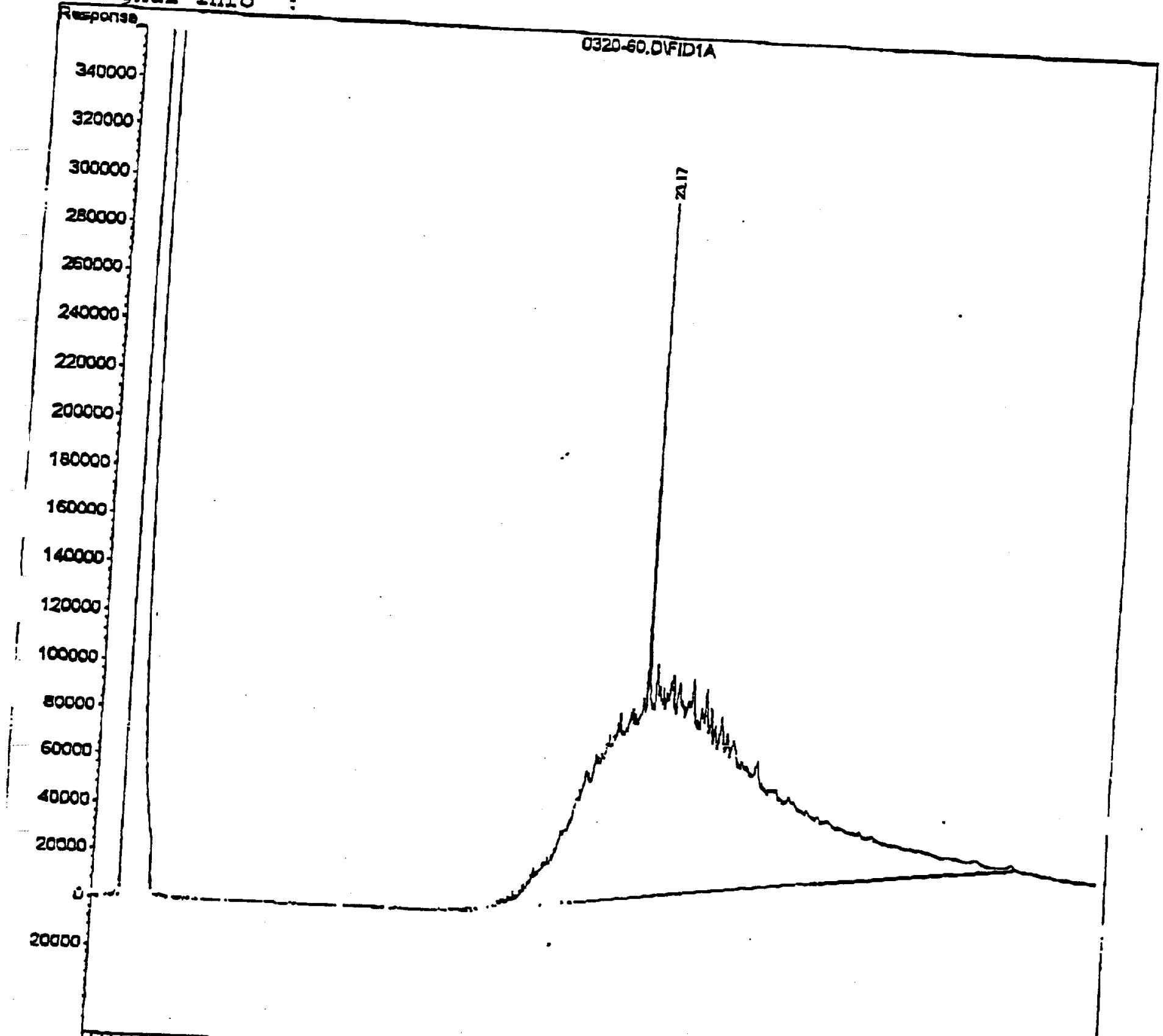




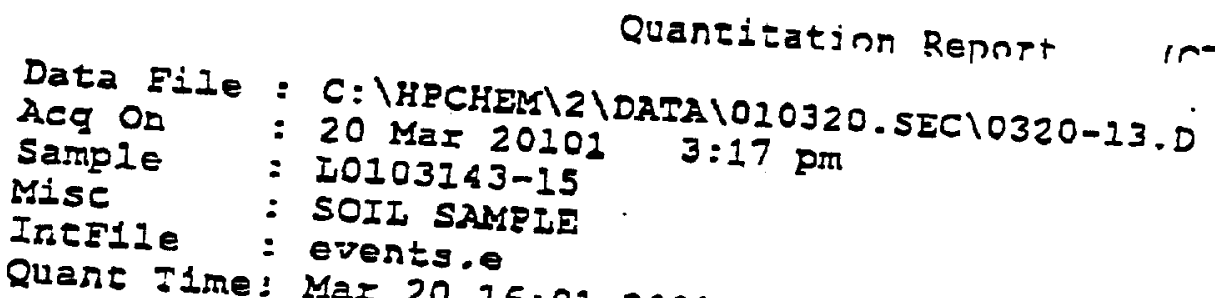

\section{Quanzization Repnrt}

(ก) จ-4.

A.cg on

: 20 Mar 20102 DATA 010320. SEC10320-13.D

- 20103743-15 3:17 pm

2

Ouant atme: Mants.e

quant Method

Title

Last Update

Response via

2001

Quant Results Eile: TPO10IR2.RES

Vial: 8

Operator: ccs

Inst : Sejmot:

Multipls: 1.00

: Initial Cal 15:10:47 2001

Dascg Meth : IR9907RI.M

Volume Inj. :

Sigral Phase:

Signal Info:

Compound

$1 !$

Target Compounds

2) $\mathrm{G}$ Gasolinane

3) H Diesel

\section{R.I.} Resporse

Cone Units

23.38

11.70

21.61
6915938
312881
16349528

16349528
$0.103 \mathrm{mg} / \mathrm{mlm}-$
$0.140 \mathrm{mg} / \mathrm{ml} \mathrm{ND}$
$0.250 \mathrm{mg} / \mathrm{ml}$ 


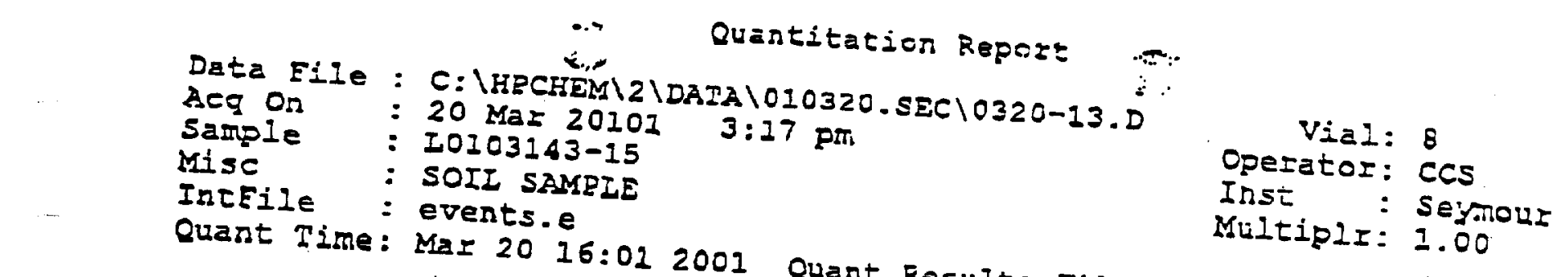

Quant Method :

Titie

last Update : Wed Jan 10 yeyour Ercrt TPH

Response via : Mulriple Level 10:47 2001

DataAcq Meth : Tp9907RI.M
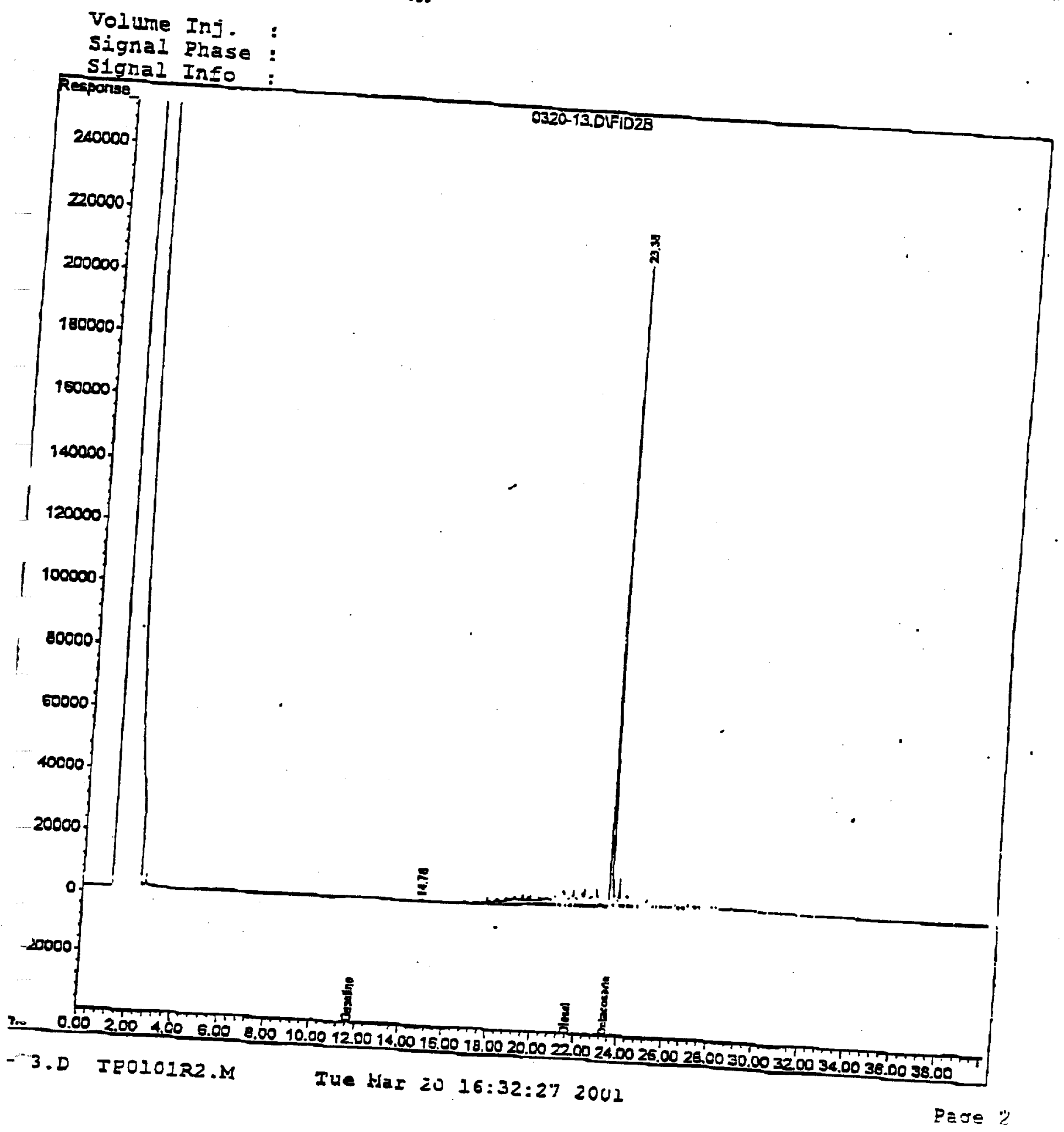


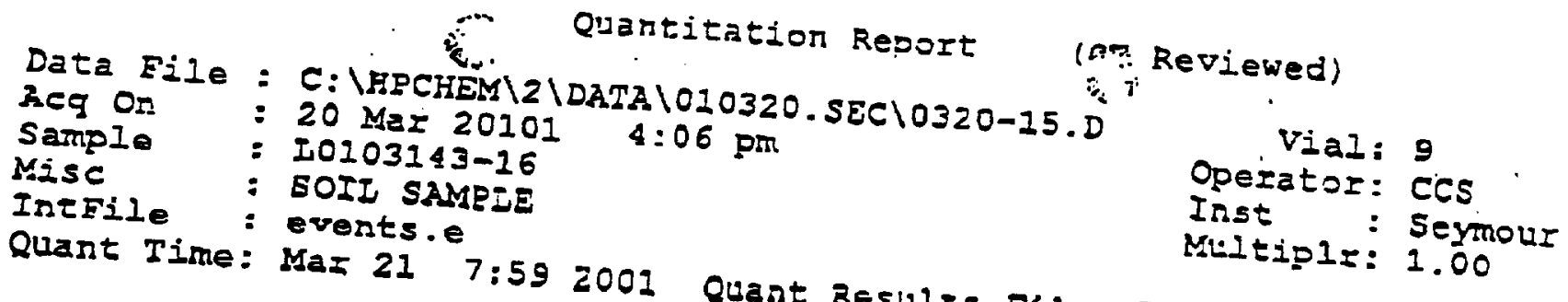

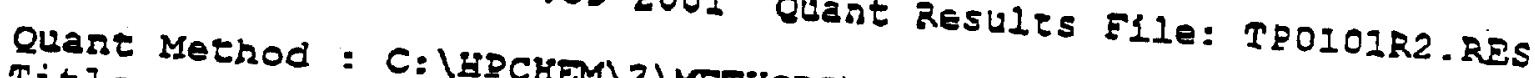

Title

Last Update: Fed Jan 10 15:10:47 2001

Datakcq Meth: Initial Calibration

Volune Inj.:
Stgnal Phase:
Slgaal Info :

Compound

1)

Target Compounds

2) H Octacosane

3) II Diesel
R.T.

Resporse

Cone Units

23.40

11.70

23.61
6926564

2253688

17431596
$0,103 \mathrm{mg} / \mathrm{mlm}$

$0.178 \mathrm{mg} / \mathrm{ml}$

$0.265 \mathrm{mg} / \mathrm{ml}$ 


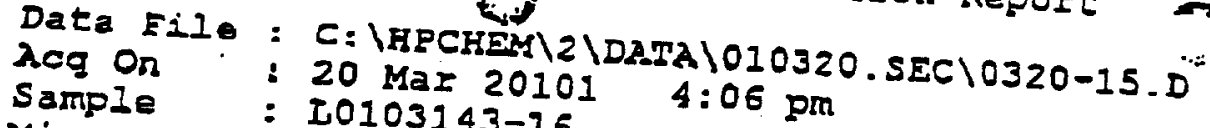

Mise

- SOII SAMELE

Inefile : events.e

Quant Time: Mar 21

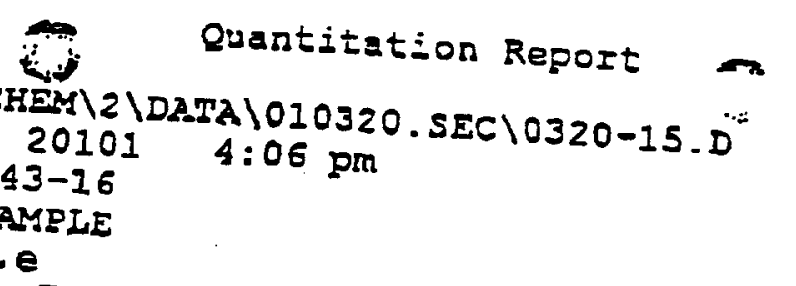
Vial: 9
Opezator: cCs
Inst i Seymou=
Multipls: 1.00

Titie

Last Update : Wed Jan 20 15:10:472001

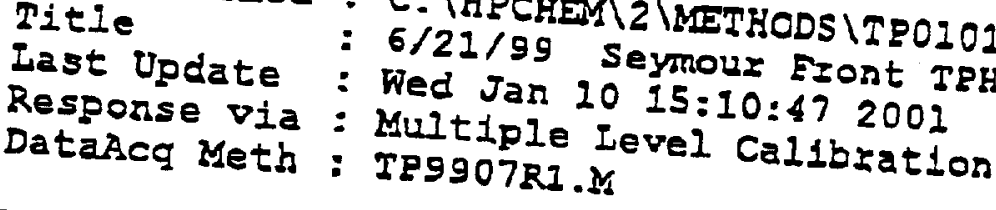

Volame Inj.

Signal Phase:

Signal Info:

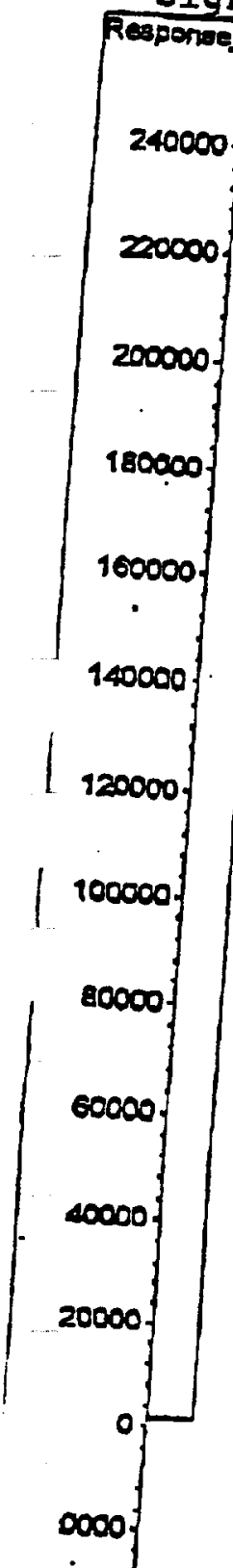

\author{
:

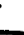
2. $M$




\section{TIER I REVIEW}

\section{GENERAL INFORMATION}

1. Project Name or Sample Delivery Group (SDG): $\mathrm{V} / 034$

2. Date Samples taken: $3 / 13 / 01$

\begin{tabular}{|c|c|c|}
\hline SAMPLE NUMBER & DATE RECEIVED & DATE ANALYZED \\
\hline $3205 B-10$ & $3 / 14 / 01$ & $3 / 20$ \\
\hline $32053-11$ & & $3 / 20$ \\
\hline $32058-12$ & & $3 / 20$ \\
\hline $3205 \beta-13$ & & $3 / 20$ \\
\hline 3205014 & 1 & $3 / 20$ \\
\hline $320,8-15$ & $\psi$ & $3 / 20$ \\
\hline $3205 B-E B-1$ & & $3 / 16$ \\
\hline $3205 p-1$ & & $3 / 20$ \\
\hline $320 J p-2 \mathrm{~ms} / \mathrm{mon}$ & & $3 / 20$ \\
\hline $3205 B-3$ & & $3 / 20$ \\
\hline $32058-4$ & & $3 / 20$ \\
\hline $3205 B-5$ & & $3 / 20$ \\
\hline $32058-6$ & & $3 / 20$ \\
\hline $32058-7$ & & $3 / 20$ \\
\hline $3205 p 8$ & & $3 / 20$ \\
\hline $3205 \beta-9$ & $d$ & $3 / 20$ \\
\hline & & \\
\hline & & \\
\hline & & \\
\hline
\end{tabular}


3. Date of Review: $3 / 26 / 0 /$

4. Chain of Custody (COC):
Completed?
ATYes $\square$ No
Legible? QYes $\square$ No

5. Is a cover letter/case narrative attached? \& Yes $\square$ No

If 'yes,' has it been reviewed for significant problems? Yes $\square$ No $\square$ NA

Comments: nore repated

6. Analyses requested (Attach COC, Sample Request Form, and lab data packet to this review):

$\begin{array}{ll}\square \text { Total VOCs } & \text { Total BNA } \square \text { Total Metals } \square \text { Radionuclides } \\ \square \text { TCLP VOCs } & \square \text { TCLP BNA } \square \text { TCLP Metals \& } \text { TPH }^{-} \\ \square \text { PCBs } & \square \text { Other: }\end{array}$

7. Were all requested analyses performed on all samples? 7 Yes $\square$ No

8. Temperature on cooler: $\underline{Z}^{\circ} \mathrm{C}$ (parameters: $4^{\circ} \mathrm{C} \pm 2^{\circ}$ ) or $\square \mathrm{NA}$

10. Refer to Table 1. Was the proper preservation used? \& Yes $\square$ No $\square$ NA If 'no,' then explain: 


\section{TOTAL PETROLEUM HYDROCARBONS (TPH)}

\begin{tabular}{|c|c|c|c|c|c|}
\hline PARAMETER & $\begin{array}{l}\text { EXTRACTION } \\
\text { HOLD TIME } \\
\end{array}$ & $\begin{array}{c}\text { ANALYSIS } \\
\text { HOLD TIME } \\
\end{array}$ & $\begin{array}{l}\text { DAYS } \\
\text { HELD } \\
\end{array}$ & $\begin{array}{c}\text { PASS } \\
\text { Y/N } \\
\end{array}$ & $\begin{array}{c}\text { SAMPLES } \\
\text { NOT PASSING } \\
\end{array}$ \\
\hline $\begin{array}{l}\text { Total TPH } \\
\text { EPA Method } \\
8015 \\
\text { Modified }\end{array}$ & $\begin{array}{l}\text { Liquids }-14 \text { days } \\
\text { Soils }-14 \text { days } \\
\text { Oil - } 14 \text { days }\end{array}$ & NA & 7 & & \\
\hline $\begin{array}{l}\text { Total TPH } \\
\text { EPA Method } \\
8015 \\
\text { Modified } \\
\end{array}$ & NA & $\begin{array}{l}\text { Liquids }-40 \\
\text { days } \\
\text { Soils - } 40 \text { days } \\
\text { Oil - } 40 \text { days }\end{array}$ & 0 & & \\
\hline \multicolumn{6}{|c|}{ 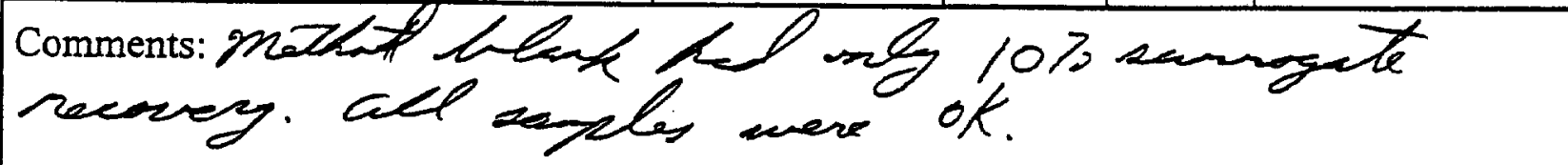 } \\
\hline
\end{tabular}

Was TPH digestion done within the hold time limit? \Yes $\square$ No

Were analyses run within the hold time limit? $\square$ Yes $\square$ No
A. $\mathrm{TPH}$ reported as: $\mathrm{mg} / \mathrm{Kg}$ or $\square \mathrm{ug} / \mathrm{Kg}$ Other:
B. Hits above detection level found in LB, RBS, FB, RB, or other QA samples? Yes No If 'yes,' explain:

C. Did laboratory report indicate any problems? $\square$ Yes $\not$ No If 'yes,' explain: 


\section{SUMMMARY}

12. Laboratory log-in report check for completeness and errors. Yes $\square$ No

13. Are all field forms are present and complete? Wryes $\square$ No

14. Does the report forms inventory include all CLP or LP-like forms? \Yes $\square$ No

15. Are the reporting levels at the appropriate level? 8 Yes $\square$ No

If 'no,' list the exceptions below:

\begin{tabular}{|l|c|c|c|c|}
\hline SAMPLE ID & PARAMETER & REPORTING & ACTUAL & COMMENTS \\
& ANALYSIS & LEVELS & LEVEL & \\
\hline & & & & \\
\hline & & & & \\
\hline & & & & \\
\hline
\end{tabular}

Was the sample count/type consistent with the COC? Yes $\square$ No

Were the results reported for both the field and laboratory QC samples? 8 Yes $\square$ No

Is the analysis count/type consistent with the COC? Yes $\square$ No

Was the correct sample matrix used for each sample? $\square$ No

Certificates of Analysis (COAs) checked for completeness? Yes $\square$ No

Condition-upon-receipt variance form included? $\square$ Yes $\square$ No $/$ N/A

Did the deliverable meet the overall objectives of the project? \Yes $\square$ No

16. Are all signatures in place? On COC? YYes $\square$ No On Data pkg.? Yes $\square$ No 
17. Explanation for any problems:

18. Overall Comments:

19. Reviewed by: thes

20. Task Manager or TPO
Date: $3 / 26 / 01$ Signature

Date:

Signature 
APPENDIX E

\section{COMMENT REVIEW DOCUMENTATION}


THIS PAGE INTENTIONALLY LEFT BLANK 


\section{DOCUMENT REVIEW SHEET}

\begin{tabular}{|c|c|c|c|c|}
\hline \multicolumn{5}{|c|}{$\begin{array}{l}\text { 1. Document Title/Number: Draft Closure Report for Corrective Action Unit } 230 \\
\text { Unit 320: Area 22 Desert Rock Airport Strainer Box, Nevada Test Site, Nevada } \\
\text { 2. Document Date: May } 2001 \\
\text { 3. Revision Number: } 0 \\
\text { 4. Originator/Organization: BN } \\
\text { 5. Date Comments Due: } \\
\text { 6. Reviewer/Organization: NDEP }\end{array}$} \\
\hline $\begin{array}{l}\text { 7. Comment } \\
\text { Number/ } \\
\text { Location }\end{array}$ & $\begin{array}{l}8 . \\
\text { Type }\end{array}$ & 9. Comment & 10. Comment Response & $\begin{array}{c}14 . \\
\text { Accept }\end{array}$ \\
\hline $\begin{array}{l}\text { 1. Executive } \\
\text { Summary, page } x \text {, } \\
\text { second paragraph }\end{array}$ & $\mathrm{M}$ & $\begin{array}{l}\text { "...Therefore the National Nuclear Security } \\
\text { Administration Nevada Operations..." } \\
\text { Add the acronym (NNSA/NV) here. }\end{array}$ & $\begin{array}{l}\text { The Executive Summary is considered a } \\
\text { "stand alone" document. Since the } \\
\text { phrase "National Nuclear Security } \\
\text { Administration Nevada Operations } \\
\text { Office" appears only once in the } \\
\text { Executive Summary, an acronym is not } \\
\text { required. }\end{array}$ & $\mathrm{N}$ \\
\hline
\end{tabular}


THIS PAGE INTENTIONALLY LEFT BLANK 
DISTRIBUTION LIST 
THIS PAGE INTENTIONALLY LEFT BLANK 


\section{DISTRIBUTION LIST}

*Provide copy of initial distribution or Revision 0; remainder of list gets Revision 0 if approved without changes. The entire list receives Revision 1 , if issued.

\section{Nevada Division of Environmental Protection}

Paul Liebendorfer

Bureau of Federal Facilities

2 (Controlled)*

Division of Environmental Protection

333 W. Nye Lane, Room 138

Carson City, NV 89706-0851

Mike McKinnon, Las Vegas Office

Bureau of Federal Facilities

1 (Controlled)*

Division of Environmental Protection

555 E. Washington, Suite 4300

Las Vegas, NV 89101-1049

\section{U.S. Department of Energy}

Janet Appenzeller-Wing

Environmental Restoration Division

1 (Uncontrolled)*

U.S. Department of Energy

National Nuclear Security Administration

Nevada Operations Office

P.O. Box $98518 \mathrm{M} / \mathrm{S} 505$

Las Vegas, NV 89193-8518

Sabine Curtis

Environmental Restoration Division

1 (Uncontrolled)*

U.S. Department of Energy

National Nuclear Security Administration

Nevada Operations Office

P.O. Box $98518 \mathrm{M} / \mathrm{S} 505$

Las Vegas, NV 89193-8518 


\section{DISTRIBUTION LIST (Continued)}

\section{U.S. Department of Energy (continued)}

Sabrina Lawrence

Environmental Restoration Division

1 (Controlled)*

U.S. Department of Energy

National Nuclear Security Administration

Nevada Operations Office

P.O. Box $98518 \mathrm{M} / \mathrm{S} 505$

Las Vegas, NV 89193-8518

U.S. Department of Energy

1 (Uncontrolled,

Office of Scientific and Technical Information electronic copy)

P.O. Box 62

Oak Ridge, TN 37831-0062

U.S. Department of Energy

1 (Uncontrolled)

National Nuclear Security Administration

Nevada Operations Office

Technical Information Resource Center

P.O. Box $98518 \mathrm{M} / \mathrm{S} 505$

Las Vegas, NV 89193-8518

Manager, Southern Nevada

1 (Controlled) \&

FFACO Public Reading Room

1 (Uncontrolled)

P.O. Box 98521, M/S NLV040

Las Vegas, NV 89193-8521

Manager, Northern Nevada

1 (Uncontrolled)

FFACO Public Reading Room

Nevada State Library and Archives, Federal Publications

100 North Stewart Street

Carson City, NV 89701-4284

\section{Bechtel Nevada}

Correspondence Control

1 (Uncontrolled)*

Bechtel Nevada

P.O. Box $98521 \mathrm{M} / \mathrm{S}$ NLV008

Las Vegas, NV 89193-8521 


\section{DISTRIBUTION LIST (Continued)}

\section{Bechtel Nevada (continued)}

Environmental Management Library

Bechtel Nevada

P.O. Box $98521 \mathrm{M} / \mathrm{S}$ NLV080

Las Vegas, NV 89193-8521

Dennis Gustafson

1 (Uncontrolled)*

Bechtel Nevada

P.O. Box $98521 \mathrm{M} / \mathrm{S}$ NTS306

Las Vegas, NV 89193-8521

Ann Heidema

1 (Uncontrolled)*

Bechtel Nevada

P.O. Box $98521 \mathrm{M} / \mathrm{S}$ NLV022

Las Vegas, NV 89193-8521

Wayne Johnson

1 (Uncontrolled)*

Bechtel Nevada

1 (Uncontrolled)*

P.O. Box $98521 \mathrm{M} / \mathrm{S}$ NTS306

Las Vegas, NV 89193-8521

Steve Nacht

1 (Uncontrolled)*

Bechtel Nevada

P.O. Box $98521 \mathrm{M} / \mathrm{S}$ NTS306

Las Vegas, NV 89193-8521

Dan Tobiason

1 (Uncontrolled)*

Bechtel Nevada

P.O. Box $98521 \mathrm{M} / \mathrm{S}$ NTS306

Las Vegas, NV 89193-8521

\section{IT Corporation}

Lynn Kidman

1 (Uncontrolled)*

IT Corporation

P.O. Box 93838, M/S 439

Las Vegas, NV 89193-3838 


\section{DISTRIBUTION LIST (Continued)}

\section{IT Corporation (continued)}

Dustin Wilson

1 (Uncontrolled)*

IT Corporation

P.O. Box 93838, M/S NLV439

Las Vegas, NV 89193-3838

IT FFACO Support Office

1 (Controlled)

IT Corporation

P.O. Box 93838, M/S 439

Las Vegas, NV 89193-3838 